\title{
Enantioselective Total Synthesis of Cotylenin A
}

Masahiro Uwamori, Ryunosuke Osada, Ryoji Sugiyama, Kotaro Nagatani, and Masahisa Nakada*

Department of Chemistry and Biochemistry, Graduate School of Advanced Science and Engineering, Waseda University, 3-4-1 Ohkubo, Shinjuku-ku, Tokyo 169-8555, Japan

Supporting Information

Table of Contents

General Information

Experimental Procedure

Prepararion of A-Ring Fragment 16

Prepararion of C-Ring Fragment 7

Enantioselective Total Synthesis of Cotylenol-

Preparation of Sugar Part fragment

Enantioselective Total Synthesis of Cotylenin A 


\section{General Information}

${ }^{1} \mathrm{H}$ and ${ }^{13} \mathrm{C}$ NMR spectra were recorded on a JEOL AL-400 or a JEOL ECZ 500 or JEOL ECX 500 or Bruker AVANCE 600 spectrometer. ${ }^{1} \mathrm{H}$ and ${ }^{13} \mathrm{C}$ chemical shifts are reported in ppm downfield from tetramethylsilane (TMS, $\delta$ scale) with the solvent resonances as internal standards. The following abbreviations were used to explain the multiplicities: s, singlet; $d$, doublet; t, triplet; q, quartet; m, multiplet; br, broad; dd, double doublet. IR spectra were recorded on a JASCO FT/IR-8300. Mass spectra and elemental analyses were provided at the Materials Characterization Central Laboratory, Waseda University. All reactions were carried out under an argon atmosphere with dry, freshly distilled solvents under anhydrous conditions, unless otherwise noted. All reactions were monitored by thin-layer chromatography carried out on 0.25 $\mathrm{mm}$ E. Merck silica gel plates (60F-254) using UV light as visualizing agent and phosphomolybdic acid and heat as developing agents. Kanto Chemical Silica Gel 60N (spherical, neutral, $63-210 \mu \mathrm{m}$ or $40-50 \mu \mathrm{m}$ partial size) was used for flash column chromatography. Preparative thin-layer chromatography (PTLC) separations were carried out on self-made $0.3 \mathrm{~mm}$ E. Merck silica gel plates (60F-254).

Materials. THF and $\mathrm{Et}_{2} \mathrm{O}$ were distilled from sodium/benzophenone ketyl. Toluene was distilled from sodium. $\mathrm{MeOH}$ was distilled with a small amount of magnesium and $\mathrm{I}_{2}$. Benzene and $\mathrm{MeCN}$ were distilled from $\mathrm{CaH}_{2}$, and all other reagents were purchased from Aldrich, Nacalai, TCI, FIJIFILM Wako, or Kanto Chemical Co. Ltd. 


\section{Prepararion of A-Ring Fragment 16}

\section{(E)-1-mesitylsulfonylhept-5-en-2-one (12a)}<smiles>C/C=C/CCC(=O)CS(C)(=O)=O</smiles>

To a stirred solution of $\mathrm{MeSO}_{2} \mathrm{Mes}(24.3 \mathrm{~g}, 122.7 \mathrm{mmol})$ in THF $(246 \mathrm{~mL})$ was added $n$-BuLi (1.55 M solution in hexane, $158 \mathrm{~mL}, 245.5 \mathrm{mmol})$ at $0{ }^{\circ} \mathrm{C}$, and the resultant solution was gradually warmed up to room temperature and was stirred for $1 \mathrm{~h}$. To the reaction mixture was added a solution of ethyl $(E)$-hex-4-enoate $12(17.4 \mathrm{~g}, 122.7 \mathrm{mmol})$ in THF $(123 \mathrm{~mL})$ at $-78{ }^{\circ} \mathrm{C}$, and the resultant mixture was stirred at $0{ }^{\circ} \mathrm{C}$ for $1 \mathrm{~h}$. The reaction mixture was quenched with saturated aqueous $\mathrm{NH}_{4} \mathrm{Cl}$ solution $(300 \mathrm{~mL})$, and the aqueous layer was extracted with ethyl acetate $(300 \mathrm{~mL} \times 3)$. The combined organic layer was washed with brine $(800 \mathrm{~mL})$, dried over $\mathrm{Na}_{2} \mathrm{SO}_{4}$, and concentrated under reduced pressure. The residue was purified by flash column chromatography (hexane/ethyl acetate $=10 / 1)$ to afford 12a $(34.9 \mathrm{~g}, 118.7 \mathrm{mmol}, 97 \%)$ as a white solid: $\mathrm{R}_{\mathrm{f}}=0.50$ (hexane/ethyl acetate $\left.=2 / 1\right) ; \mathrm{mp} 51-52{ }^{\circ} \mathrm{C} ;{ }^{1} \mathrm{H}$ NMR $\left(500 \mathrm{MHz}, \mathrm{CDCl}_{3}\right) \delta 6.98$ (s, 2H), 5.50-5.42 (m, 1H), 5.38-5.33 (m, 1H), $4.12(\mathrm{~s}, 2 \mathrm{H}), 2.78(\mathrm{t}, J=7.0 \mathrm{~Hz}, 2 \mathrm{H}), 2.62(\mathrm{~s}, 6 \mathrm{H})$, 2.31 (s. 3H), 2.28-2.20 (m, 2H), $1.62(\mathrm{dd}, J=6.0,1.5 \mathrm{~Hz}, 3 \mathrm{H}) ;{ }^{13} \mathrm{C}\left(125 \mathrm{MHz}, \mathrm{CDCl}_{3}\right) \delta 198.1$, $144.1,140.2,133.0,132.6,128.8,126.7,66.9,44.8,26.3,23.0,21.2,18.0$; IR (ATR) $v_{\max } 3022$, 2971, 2939, 2854, 1712, 1600, 1453, 1396, 1310, 1133, 961, 854, 736, $579 \mathrm{~cm}^{-1}$; HRMS (ESI) $[\mathrm{M}+\mathrm{Na}]$ calcd for $\mathrm{C}_{16} \mathrm{H}_{22} \mathrm{O}_{3} \mathrm{NaS} 317.1182$, found 317.1182 .

\section{(E)-1-diazo-1-mesitylsulfonylhept-5-en-2-one (9)}<smiles>C/C=C/CCC(=O)C(=N)OS(C)(=O)=O</smiles>

To a stirred suspension of 12a $(10.2 \mathrm{~g}, 34.8 \mathrm{mmol})$ and $\mathrm{Et}_{3} \mathrm{~N}(14.5 \mathrm{~mL}, 104 \mathrm{mmol})$ in $\mathrm{CH}_{3} \mathrm{CN}(174 \mathrm{~mL})$ was added $\mathrm{TsN}_{3}\left(0.75 \mathrm{M}\right.$ in $\left.\mathrm{CH}_{3} \mathrm{CN}, 69.6 \mathrm{~mL}, 52.2 \mathrm{mmol}\right)$ at $0{ }^{\circ} \mathrm{C}$. The reaction mixture was stirred at $0{ }^{\circ} \mathrm{C}$ for $24 \mathrm{~h}$, and then quenched with saturated aqueous $\mathrm{NaHCO}_{3}$ solution $(200 \mathrm{~mL})$. The aqueous layer was extracted with ethyl acetate $(250 \mathrm{~mL} \times 3)$, and the combined organic layer was washed with brine $(200 \mathrm{~mL})$, dried over $\mathrm{Na}_{2} \mathrm{SO}_{4}$, and concentrated under reduced pressure. The residue was purified by flash column chromatography (hexane/ dichloromethane $=3 / 1$ to hexane/ ethyl acetate $=10 / 1)$ to afford $9(10.3 \mathrm{~g}, 34.1 \mathrm{mmol}, 98 \%)$ as a yellow crystal: $\mathrm{R}_{\mathrm{f}}=0.60$ (hexane/ethyl acetate $\left.=2 / 1\right) ; \mathrm{mp} 89-91{ }^{\circ} \mathrm{C} ;{ }^{1} \mathrm{H} \mathrm{NMR}\left(500 \mathrm{MHz}, \mathrm{CDCl}_{3}\right)$ 
$\delta 7.00(\mathrm{~s}, 2 \mathrm{H}), 5.38-5.25(\mathrm{~m}, 1 \mathrm{H}), 5.25-5.15(\mathrm{~m}, 1 \mathrm{H}), 2.65(\mathrm{~s}, 6 \mathrm{H}), 2.46(\mathrm{t}, J=7.5 \mathrm{~Hz}, 2 \mathrm{H}), 2.31$ $(\mathrm{s}, 3 \mathrm{H}), 2.18(\mathrm{~d}, J=7.0 \mathrm{~Hz}, 1 \mathrm{H}), 2.16(\mathrm{~d}, J=7.0 \mathrm{~Hz}, 1 \mathrm{H}), 1.56(\mathrm{~d}, J=6.5 \mathrm{~Hz}, 3 \mathrm{H}) ;{ }^{13} \mathrm{C}(125 \mathrm{MHz}$, $\left.\mathrm{CDCl}_{3}\right) \delta 188.7,144.3,140.3,135.2,132.7,128.5,126.8,39.0,26.8,22.6,21.2,17.9$; IR (ATR) $v_{\max } 2916,2109,1666,1599,1442,1324,1146,971,852,659,595 \mathrm{~cm}^{-1}$; HRMS (ESI) [M+Na] calcd for $\mathrm{C}_{16} \mathrm{H}_{20} \mathrm{O}_{3} \mathrm{~N}_{2} \mathrm{NaS} 343.1087$, found 343.1088.

\section{(1S,5S,6R)-1-mesitylsulfonyl-6-methylbicyclo[3.1.0]hexan-2-one (8)}

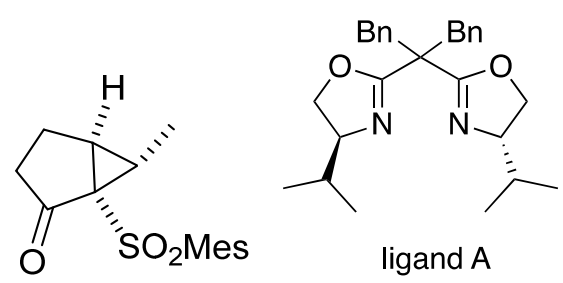

To a stirred solution of ligand $\mathrm{A}(3.76 \mathrm{~g}, 9.00 \mathrm{mmol})$ in toluene $(1000 \mathrm{~mL})$ was added $\mathrm{CuPF}_{6}\left(\mathrm{CH}_{3} \mathrm{CN}\right)_{4}(2.24 \mathrm{~g}, 6.00 \mathrm{mmol})$ at room temperature. The reaction mixture was stirred at room temperature for $2 \mathrm{~h}$, and then $9(64.0 \mathrm{~g}, 200 \mathrm{mmol})$ was added to the reaction mixture. The reaction mixture was warmed up to $60{ }^{\circ} \mathrm{C}$ and stirred for $24 \mathrm{~h}$, and quenched with saturated aqueous $\mathrm{NH}_{4} \mathrm{Cl}$ solution $(500 \mathrm{~mL})$. The aqueous layer was extracted with ethyl acetate $(700$ $\mathrm{mL} \times 3$ ), and the combined organic layer was washed with brine $(1000 \mathrm{~mL})$, dried over $\mathrm{Na}_{2} \mathrm{SO}_{4}$, and concentrated under reduced pressure. The residue was purified by flash column chromatography (hexane/ethyl acetate $=2 / 1)$ to afford $8(52.5 \mathrm{~g}, 179.7 \mathrm{mmol}, 86 \%, 86 \%$ ee $)$ as a white solid. The enantiomeric excess was determined by HPLC analysis $(220 \mathrm{~nm})$ with a chiral HPLC column (DAICEL CHIRALCEL IC-3, 9\% 2-propanol in hexane, $1.0 \mathrm{~mL} / \mathrm{min}$ ). The retention times for 8 and its enantiomer were 21.3 and $25.1 \mathrm{~min}$, respectively. This solid was recrystallized from ethyl acetate and hexane to afford enatio-enriched 8 (44.3 g, $151.7 \mathrm{mmol}, 99 \%$ ee): $\mathrm{R}_{\mathrm{f}}=0.20$ (hexane/ethyl acetate $\left.=4 / 1\right) ; \mathrm{mp} 155-157{ }^{\circ} \mathrm{C} ;{ }^{1} \mathrm{H}$ NMR $\left(500 \mathrm{MHz}, \mathrm{CDCl}_{3}\right) \delta 6.93$ (s, 2H), $2.81(\mathrm{t}, J=5.5 \mathrm{~Hz}, 1 \mathrm{H}), 2.72(\mathrm{~s}, 6 \mathrm{H}), 2.28(\mathrm{~s}, 3 \mathrm{H}), 2.20-2.03(\mathrm{~m}, 4 \mathrm{H}), 1.94$ (quin, $J=$ $5.5 \mathrm{~Hz}, 1 \mathrm{H}), 1.73(\mathrm{~d}, J=6.0 \mathrm{~Hz}, 3 \mathrm{H}) ;{ }^{13} \mathrm{C}\left(125 \mathrm{MHz}, \mathrm{CDCl}_{3}\right) \delta 204.9,143.2,140.6,132.8,132.1$, 60.1, 40.1, 33.7, 31.0, 23.0, 21.2, 21.1, 12.5; IR (ATR) $v_{\max } 2943,1716,1603,1459,1303,1271$, $1140,1027,851,663,599,537 \mathrm{~cm}^{-1}$; HRMS (ESI) [M+Na] calcd for $\mathrm{C}_{16} \mathrm{H}_{20} \mathrm{O}_{3} \mathrm{NaS} 315.1025$, found 315.1024; $[\alpha]_{\mathrm{D}}^{25}-99\left(\right.$ c $\left.0.50, \mathrm{CHCl}_{3}\right)$. 


\section{Preparation of 8 (racemate)}<smiles>CC(C)(C1=NCCO1)C1=NCCO1</smiles>

ligand $B$

To a stirred solution of ligand B $(4.3 \mathrm{mg}, 0.0234 \mathrm{mmol})$ in toluene $(3.1 \mathrm{~mL})$ was added $\mathrm{CuPF}_{6}\left(\mathrm{CH}_{3} \mathrm{CN}\right)_{4}(5.8 \mathrm{mg}, 0.0156 \mathrm{mmol})$ at room temperature. The reaction mixture was stirred for $2 \mathrm{~h}$, and then $9(100 \mathrm{mg}, 0.313 \mathrm{mmol})$ was added to the reaction mixture. The reaction mixture was warmed up to $60{ }^{\circ} \mathrm{C}$ and stirred for $24 \mathrm{~h}$. The reaction mixture was quenched with saturated aqueous $\mathrm{NH}_{4} \mathrm{Cl}$ solution $(10 \mathrm{~mL})$, and the aqueous layer was extracted with ethyl acetate $(10$ $\mathrm{mL} \times 3)$. The combined organic layer was washed with brine $(20 \mathrm{~mL})$, dried over $\mathrm{Na}_{2} \mathrm{SO}_{4}$, and concentrated under reduced pressure. The residue was purified by flash column chromatography (hexane/ethyl acetate $=2 / 1)$ to afford 8 (racemate) $(26 \mathrm{mg}, 29 \%)$ as a white crystal: $\mathrm{mp} 135$ $137^{\circ} \mathrm{C}$.

\section{HPLC analysis of 8}

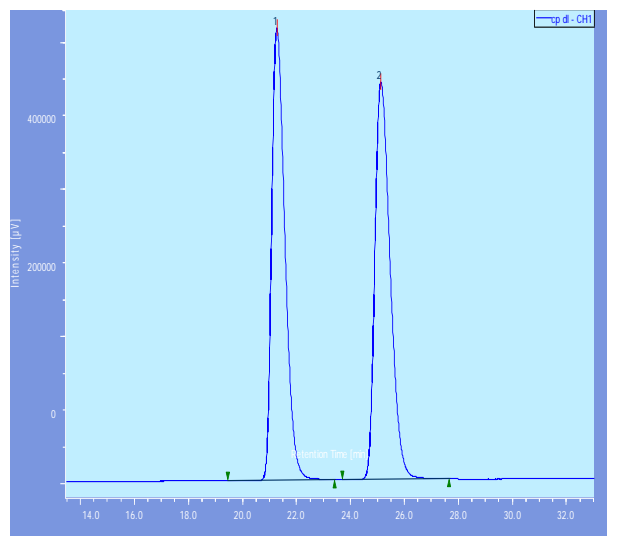

8 (racemate)

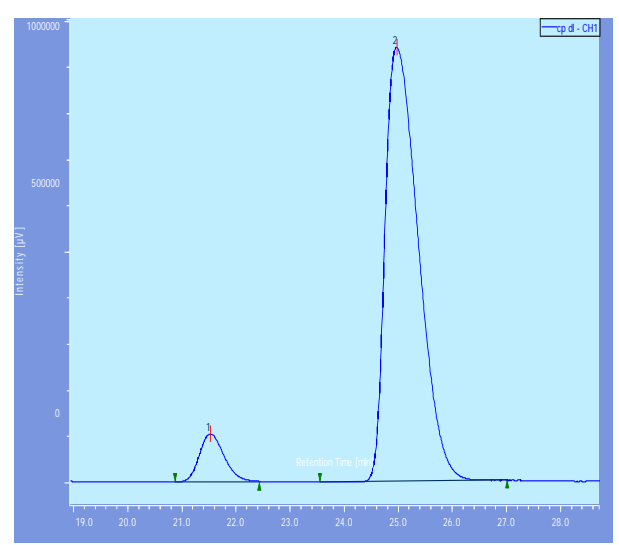

\begin{tabular}{|c|c|c|c|c}
\hline No & tR & Area & Heigh & Area (\%) \\
\hline 1 & 21.267 & 20772401 & 612280 & 49.625 \\
\hline 2 & 25.117 & 21086233 & 537665 & 50.375 \\
\hline
\end{tabular}

8 (the reaction product)

\begin{tabular}{|c|c|c|c|c|}
\hline No & tR & Area & Heigh & Area (\%) \\
\hline 1 & 21.525 & 3285033 & 103684 & 7.615 \\
\hline 2 & 24.967 & 39855564 & 939522 & 92.385 \\
\hline
\end{tabular}




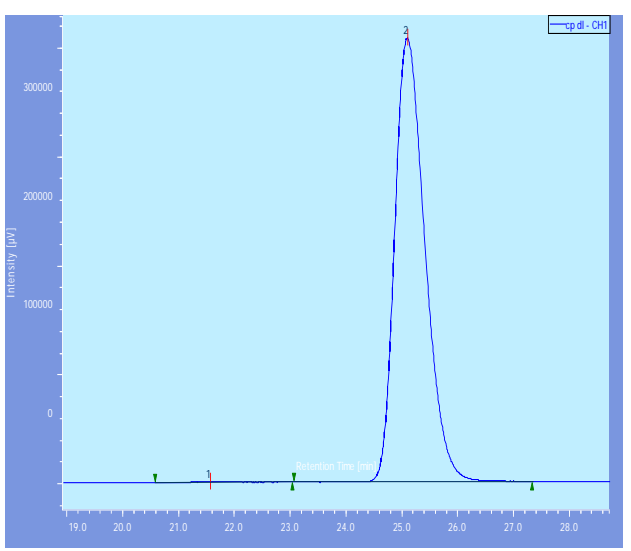

8

(after recrystallization)

\begin{tabular}{|c|c|c|c|c|}
\hline No & tR & Area & Heigh & Area (\%) \\
\hline 1 & 21.567 & 11666 & 307 & 0.077 \\
\hline 2 & 25.092 & 15220104 & 406164 & 99.923 \\
\hline
\end{tabular}

(R)-2-((1S,2S)-2-mesitylsulfonyl-3-oxocyclopentyl)propanenitrile (13)<smiles>COS(=O)(=O)[C@H]1C(=O)CC[C@@H]1[C@H](C)C#N</smiles>

To a stirred solution of $8(23.8 \mathrm{~g}, 81.4 \mathrm{mmol})$ in DMSO $(407 \mathrm{~mL})$ was added $\mathrm{NaCN}$ $(7.98 \mathrm{~g}, 162.9 \mathrm{mmol})$ at room temperature. The mixture was warmed at $80{ }^{\circ} \mathrm{C}$ for $1 \mathrm{~h}$, and quenched with saturated aqueous $\mathrm{NaHCO}_{3}$ solution $(400 \mathrm{~mL})$. The aqueous layer was extracted with ethyl acetate $(400 \mathrm{~mL} \times 3)$, and the combined organic layer was washed with brine $(400 \mathrm{~mL})$, dried over $\mathrm{Na}_{2} \mathrm{SO}_{4}$, and concentrated under reduced pressure. The residue was purified by flash column chromatography (hexane/ethyl acetate $=2 / 1)$ to afford $13(20.1 \mathrm{~g}, 63.0 \mathrm{mmol}, 77 \%)$ as a colorless crystal: $\mathrm{R}_{\mathrm{f}}=0.50$ (hexane/ethyl acetate $\left.=1 / 1\right)$; mp $126-128{ }^{\circ} \mathrm{C} ;{ }^{1} \mathrm{H}$ NMR $(500 \mathrm{MHz}$, $\left.\mathrm{CDCl}_{3}\right) \delta 7.01(\mathrm{~s}, 2 \mathrm{H}), 3.80(\mathrm{~d}, J=10.0 \mathrm{~Hz}, 1 \mathrm{H}), 3.68-3.58(\mathrm{~m}, 1 \mathrm{H}), 3.18-3.08(\mathrm{~m}, 1 \mathrm{H}), 2.60$ (s, $6 \mathrm{H}), 2.53-2.45(\mathrm{~m}, 2 \mathrm{H}), 2.43-2.33(\mathrm{~m}, 1 \mathrm{H}), 2.33(\mathrm{~s}, 3 \mathrm{H}), 1.85-1.73(\mathrm{~m}, 1 \mathrm{H}), 1.47$ (d, J = 7.0 Hz, $3 \mathrm{H}) ;{ }^{13} \mathrm{C}\left(125 \mathrm{MHz}, \mathrm{CDCl}_{3}\right) \delta 204.6,144.3,140.6,132.5,131.7,120.3,71.2,39.9,38.7,29.9$, 22.8. 22.3, 21.1, 15.8; IR (ATR) $v_{\max } 2975,2939,1751,1599,1560,1442,1307,1286,1127,1107$, $1045,859,741,669,645 \mathrm{~cm}^{-1}$; HRMS (ESI) [M+Na] calcd for $\mathrm{C}_{17} \mathrm{H}_{21} \mathrm{O}_{3} \mathrm{NNaS} 342.1134$, found $342.1136 ;[\alpha]_{\mathrm{D}}^{25}+50\left(c 0.50, \mathrm{CHCl}_{3}\right)$.

Racemic 13 was prepared from racemic 8 according to the procedure described above: rac-13: mp $208-210{ }^{\circ} \mathrm{C}$. 
(S)-3-((R)-1-cyanoethyl)cyclopent-1-en-1-yl diethyl phosphate (15)<smiles>CCOP(=O)(OCC)OC1=CC([C@@H](C)C#N)CC1</smiles>

To a stirred suspension of samarium $(19.7 \mathrm{~g}, 131.0 \mathrm{mmol})$ in THF $(787 \mathrm{~mL})$ was added 1,2-diiodoethane $(22.2 \mathrm{~g}, 78.7 \mathrm{mmol})$ at room temperature. The reaction mixture was stirred at room temperature for $12 \mathrm{~h}$, and to the mixture was added a solution of $\mathbf{1 3}(8.37 \mathrm{~g}, 26.2 \mathrm{mmol})$ in THF $(175 \mathrm{~mL})$ at $0{ }^{\circ} \mathrm{C}$. After $10 \mathrm{~min}, \mathrm{ClP}(\mathrm{O})(\mathrm{OEt})_{2}(7.5 \mathrm{~mL}, 52.5 \mathrm{mmol})$ was quickly added to the reaction mixture. The mixture was stirred at room temperature for $30 \mathrm{~min}$, and quenched with saturated aqueous $\mathrm{NaHCO}_{3}$ solution $(300 \mathrm{~mL})$ and then, with saturated aqueous $\mathrm{Na}_{2} \mathrm{~S}_{2} \mathrm{O}_{3}$ solution $(300 \mathrm{~mL})$. The aqueous layer was extracted with ethyl acetate $(600 \mathrm{~mL} \times 3)$. The combined organic layer was washed with brine $(800 \mathrm{~mL})$, dried over $\mathrm{Na}_{2} \mathrm{SO}_{4}$, and concentrated under reduced pressure. The residue was purified by flash column chromatography (hexane/ethyl acetate $=3 / 1$ ) to afford 15 ( $7.15 \mathrm{~g}, 26.18 \mathrm{mmol}$, quant.) as a colorless oil: $\mathrm{R}_{\mathrm{f}}=0.50$ (hexane/ethyl acetate $\left.=1 / 1\right)$ : ${ }^{1} \mathrm{H}$ NMR (500 MHz, $\left.\mathrm{CDCl}_{3}\right) \delta 5.22(\mathrm{~s}, 1 \mathrm{H}), 4.19-4.13(\mathrm{~m}, 4 \mathrm{H}), 2.90-2.85(\mathrm{~m}, 1 \mathrm{H}), 2.67-2.45(\mathrm{~m}$, $3 \mathrm{H}), 2.25-2.17(\mathrm{~m}, 1 \mathrm{H}), 1.82-1.74(\mathrm{~m}, 1 \mathrm{H}), 1.37-1.26(\mathrm{~m}, 9 \mathrm{H}) ;{ }^{13} \mathrm{C}\left(125 \mathrm{MHz}, \mathrm{CDCl}_{3}\right) \delta 152.6$ (d, $J=8.5 \mathrm{~Hz}), 121.8 .108 .7$ (d, $J=4.8 \mathrm{~Hz}), 64.6(\mathrm{~d}, J=6.0 \mathrm{~Hz}), 64.5(\mathrm{~d}, J=6.0 \mathrm{~Hz}), 45.2,31.23$, 31.18 (d, $J=4.8 \mathrm{~Hz}$ ), 24.8, 16.03, 15.97, 15.7; ${ }^{31} \mathrm{P}$ NMR (202 MHz, $\mathrm{CDCl}_{3}$, internal standard $85 \%$ $\left.\mathrm{H}_{3} \mathrm{PO}_{4}[0 \mathrm{ppm}]\right) \delta-6.1$ (s); IR (ATR) $v_{\max } 2983,2916,1656,1459,1274,1165,1021,931,852$, $819 \mathrm{~cm}^{-1}$; HRMS (ESI) [M+Na] calcd for $\mathrm{C}_{12} \mathrm{H}_{20} \mathrm{O}_{4} \mathrm{NNaP} 296.1022$, found 296.1022; $[\alpha]_{\mathrm{D}}^{25}-42$ (c $\left.0.5, \mathrm{CHCl}_{3}\right)$.

\section{(2R)-2-((1S)-2-bromo-3-oxocyclopentyl)propanenitrile (16)}<smiles>C[C@H](C#N)[C@H]1CCC(=O)C1Br</smiles>

To a stirred solution of $\mathbf{1 5}$ in THF $(271 \mathrm{~mL})$ and $\mathrm{H}_{2} \mathrm{O}(27 \mathrm{~mL})$ was added NBS (7.95 $\mathrm{g}, 44.7 \mathrm{mmol}$ ) at $0{ }^{\circ} \mathrm{C}$. The mixture was stirred at $0{ }^{\circ} \mathrm{C}$ for $30 \mathrm{~min}$, and was quenched with saturated aqueous $\mathrm{NaHCO}_{3}$ solution $(200 \mathrm{~mL})$ and then, with saturated aqueous $\mathrm{Na}_{2} \mathrm{~S}_{2} \mathrm{O}_{3}$ solution $(200 \mathrm{~mL})$. The resulting mixture was extracted with ethyl acetate $(150 \mathrm{~mL} \times 3)$, and the combined 
organic layer was washed with brine $(300 \mathrm{~mL})$, dried $\mathrm{Na}_{2} \mathrm{SO}_{4}$, and concentrated under reduced pressure. The residue was purified by flash column chromatography (hexane/ethyl acetate $=10 / 1$ ) to afford 16 (6.7 g, $31.2 \mathrm{mmol}, 82 \%$, a colorless oil) as an inseparable mixture of diastereomers. (ca. 1:0.7): $\mathrm{R}_{\mathrm{f}}=0.7$ (hexane/ethyl acetate $\left.=1 / 2\right) ;{ }^{1} \mathrm{H}$ NMR $\left(500 \mathrm{MHz}, \mathrm{CDCl}_{3}\right) \delta 4.22(\mathrm{~d}, J=11.0$ $\mathrm{Hz}, 1 \mathrm{H}), 4.16(\mathrm{~d}, J=5.5 \mathrm{~Hz}, 0.7 \mathrm{H}), 3.22(\mathrm{ddd}, J=11.0,7.0,3.5 \mathrm{~Hz}, 1 \mathrm{H}), 2.83$ (ddd, $J=14.0$, $10.5,7.0 \mathrm{~Hz}, 0.7 \mathrm{H}) 2.67-2.57(\mathrm{~m}, 1.7 \mathrm{H}), 2.42-2.21(\mathrm{~m}, 4.4 \mathrm{H}), 2.17-2.11(\mathrm{~m}, 0.7 \mathrm{H}), 1.99-1.86$ $(\mathrm{m}, 1.7 \mathrm{H}), 1.45(\mathrm{~d}, J=7.5 \mathrm{~Hz}, 3 \mathrm{H}), 1.39(\mathrm{~d}, J=7.0 \mathrm{~Hz}, 2.1 \mathrm{H}) ;{ }^{13} \mathrm{C}\left(125 \mathrm{MHz}, \mathrm{CDCl}_{3}\right) \delta 207.6$, 121.0, 119.7, 52.7, 51.7, 48.9, 45.6, 34.3, 34.9, 29.3, 27.9, 25.1, 22.8, 16.7, 15.9; IR (ATR) $v_{\max }$ 2984, 2941, 2240, 1748, 1456, 1402, 1139, 1107, 815, 654, $545 \mathrm{~cm}^{-1}$; HRMS (ESI) [M+Na] calcd for $\mathrm{C}_{8} \mathrm{H}_{10} \mathrm{ONBrNa} 237.9838$, found 237.9839; $[\alpha]_{\mathrm{D}}^{25}+36\left(c 0.50, \mathrm{CHCl}_{3}\right)$.

\section{Prepararion of C-Ring Fragment 7}<smiles>CC(C)=CCCC1(C)OC1COC(=O)c1cc([N+](=O)[O-])cc([N+](=O)[O-])c1</smiles>

$11 b^{1,2}$
1) $\mathrm{NaOH}, \mathrm{MeOH}$ $\mathrm{H}_{2} \mathrm{O}, \mathrm{rt}, 1.5 \mathrm{~h}$

2) TBSCl, imidazole $\mathrm{CH}_{2} \mathrm{Cl}_{2}, \mathrm{rt}, 1.5 \mathrm{~h}$

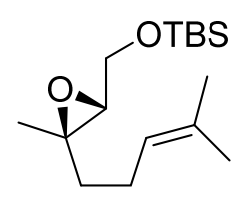

$11 \mathrm{c}$

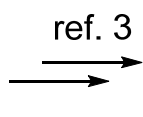

TBSO<smiles>CC(C)=CCCC(C)(C=O)CO[Mg]C(C)(C)C</smiles>

11 $99.6 \%$ ee

((2R,3R)-3-methyl-3-(4-methylpent-3-en-1-yl)oxiran-2-yl)methyl 3,5-dinitrobenzoate (11b)<smiles>CC(C)=CCCC1(C)OC1COC(=O)c1cc([N+](=O)[O-])cc([N+](=O)[O-])c1</smiles>

The enantiomeric excess of 11b was determined by HPLC analysis with a chiral HPLC column (DAICEL CHIRALCEL IC-3, 33\% 2-propanol in hexane, $1.0 \mathrm{~mL} / \mathrm{min}$ ). The retention times for $\mathbf{1 1 b}$ and its enantiomer were 44.6 and $55.6 \mathrm{~min}$, respectively. 


\section{HPLC data of 11b}

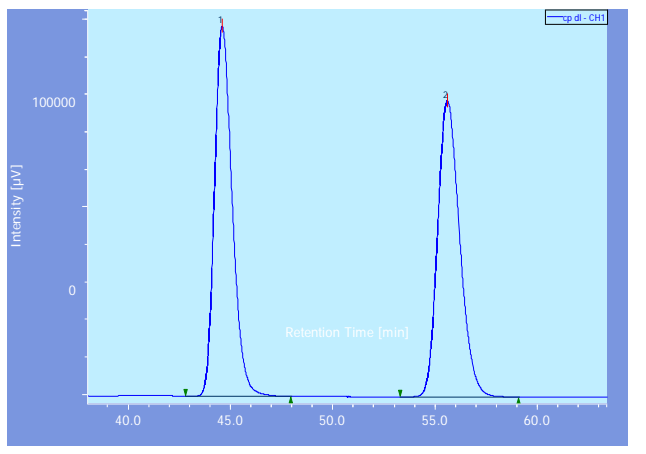

11b

(racemate)

\begin{tabular}{|c|c|c|c|c|}
\hline No & TR & Area & Height & Area (\%) \\
\hline 1 & 44.592 & 11845252 & 196926 & 50.079 \\
\hline 2 & 55.583 & 11807999 & 157572 & 49.921 \\
\hline
\end{tabular}

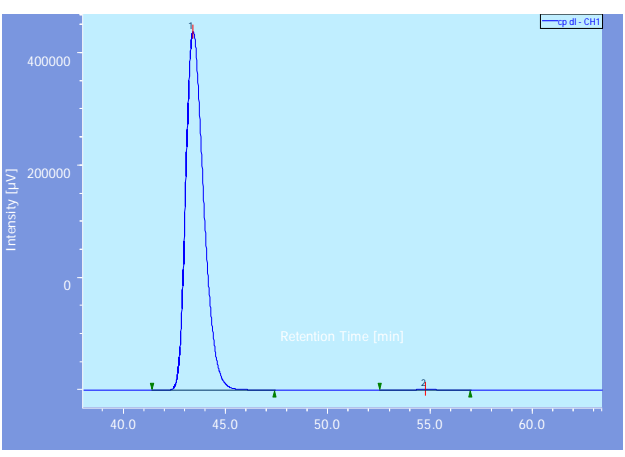

11b

(chiral)

$99.6 \%$ ee

\section{Preparation of 11c}<smiles>CC(C)=CCCC1(C)OC1COC(=O)c1cc([N+](=O)[O-])cc([N+](=O)[O-])c1</smiles>

$11 \mathrm{~b}$

$100 \%$ ee

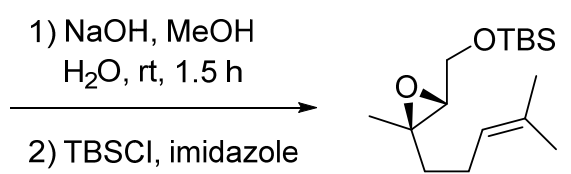

$\mathrm{CH}_{2} \mathrm{Cl}_{2}$, rt, $1.5 \mathrm{~h}$

$11 \mathrm{c}$

To a stirred solution of $\mathbf{1 1 b}(154.8 \mathrm{~g}, 443 \mathrm{mmol})$ in $\mathrm{MeOH}(1100 \mathrm{~mL})$ and $\mathrm{H}_{2} \mathrm{O}(110$ $\mathrm{mL})$ was added $\mathrm{NaOH}(20 \mathrm{~g}, 500 \mathrm{mmol})$ at room temperature. The reaction mixture was stirred at room temperature for $1.5 \mathrm{~h}$, and $\mathrm{MeOH}$ was removed under reduced pressure. The residue was extracted with ethyl acetate $(600 \mathrm{~mL} \times 3)$, and the combined organic layer was washed with brine $(800 \mathrm{~mL})$, dried $\mathrm{Na}_{2} \mathrm{SO}_{4}$, and concentrated under reduced pressure. The obtained crude epoxy alcohol was used for the next reaction without further purification.

To a stirred solution of the crude epoxy alcohol prepared as above in $\mathrm{CH}_{2} \mathrm{Cl}_{2}(1100$ 
$\mathrm{mL})$ were added imidazole $(60.3 \mathrm{~g}, 886 \mathrm{mmol})$ and $\mathrm{TBSCl}(73.5 \mathrm{~g}, 487 \mathrm{mmol})$ at $0{ }^{\circ} \mathrm{C}$. The mixture was stirred at $0{ }^{\circ} \mathrm{C}$ for $1.5 \mathrm{~h}$, and quenched with saturated aqueous $\mathrm{NaHCO}_{3}$ solution $(500$ $\mathrm{mL})$. The aqueous layer was extracted with ethyl acetate $(600 \mathrm{~mL} \times 3)$, and the combined organic layer was washed with brine $(800 \mathrm{~mL})$, dried $\mathrm{Na}_{2} \mathrm{SO}_{4}$, and concentrated under reduced pressure. The residue was purified by flash column chromatography (hexane/ethyl acetate $=20 / 1$ ) to afford 11c (108 g, $378.9 \mathrm{mmol}, 86 \%$, 2 steps) as a colorless oil: $R_{f}=0.50$ (hexane/ethyl acetate $\left.=10 / 1\right)$. The spectroscopic data of $\mathbf{1 1} \mathbf{c}$ were identical to the reported ones (reference c).

\section{(R)-5-tert-butyldimethylsilyloxymethyl-2-isopropyl-5-methylcyclopent-1-en-1-yl}

\section{trifluoromethanesulfonate (14)}

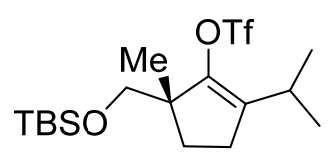

To a stirred solution of $\mathbf{1 1}(24.3 \mathrm{~g}, 85.5 \mathrm{mmol})$ in TBME $(1315 \mathrm{~mL})$ were added $\mathrm{CuCl}$ (1.30 g, $13.1 \mathrm{mmol}$ ), 2,2'-bipyridyl (3.08g, $19.7 \mathrm{mmol}$ ), TBHP (3.0 M solution in $\mathrm{CH}_{2} \mathrm{Cl}_{2}, 88 \mathrm{~mL}$, $262.9 \mathrm{mmol})$ and tert-dodecanthiol $(61.9 \mathrm{~mL}, 262.9 \mathrm{mmol})$ at room temperature. The reaction mixture was degassed and stirred at $50{ }^{\circ} \mathrm{C}$ for $12 \mathrm{~h}$. Then the reaction mixture was cooled to room temperature and the resulting mixture was passed through a Celite pad and the filtrate was concentrated under reduced pressured. The residue was purified by flash column chromatography (hexane to hexane/ethyl acetate $=100 / 1$ ). The crude ketone 11a (ca. $34.1 \mathrm{~g}$ including a small amount of impurities) was used for the next step without further purification.

To a stirred solution of the crude ketone11a (ca. $34.1 \mathrm{~g})$ and $\operatorname{PhNTf}_{2}(63.9 \mathrm{~g}, 178.9$ mmol) in THF (596 mL) was added NaHMDS $(1.9 \mathrm{M}, 94 \mathrm{~mL}, 178.9 \mathrm{mmol})$ at $0{ }^{\circ} \mathrm{C}$. The mixture was stirred at $0{ }^{\circ} \mathrm{C}$ for $1 \mathrm{~h}$, and quenched with saturated aqueous $\mathrm{NH}_{4} \mathrm{Cl}$ solution $(500 \mathrm{~mL})$. The aqueous layer was extracted with hexane $(1000 \mathrm{~mL} \times 1)$, and the combined organic layer was washed with brine $(500 \mathrm{~mL})$, dried over $\mathrm{Na}_{2} \mathrm{SO}_{4}$, filtered, and concentrated under reduced pressure. The residue was purified by flash column chromatography (hexane) to afford 14 (29.5 g, $54 \%$ (2 steps)) as a colorless oil: $\mathrm{R}_{\mathrm{f}}=0.79$ (hexane/ethyl acetate $\left.=10 / 1\right) ;{ }^{1} \mathrm{H} \mathrm{NMR}(500 \mathrm{MHz}$, $\left.\mathrm{CDCl}_{3}\right) \delta 3.51(\mathrm{~d}, J=10.0 \mathrm{~Hz}, 1 \mathrm{H}), 3.32(\mathrm{~d}, J=10.0 \mathrm{~Hz}, 1 \mathrm{H}), 2.80-2.88(\mathrm{~m}, 1 \mathrm{H}), 2.21-2.32(\mathrm{~m}$, 2H), 2.10-2.15 (m, 1H), 1.59-1.65 (m, 1H), 1.07 (s, 3H), 1.03 (d, $J=7.0 \mathrm{~Hz}, 3 \mathrm{H}), 1.01$ (d, $J=$ $7.0 \mathrm{~Hz}, 3 \mathrm{H}), 0.88$ (s, 9H), 0.03 (s, 3H), $0.02(\mathrm{~s}, 3 \mathrm{H}) ;{ }^{13} \mathrm{C} \mathrm{NMR}\left(125 \mathrm{MHz}, \mathrm{CDCl}_{3}\right) \delta 142.9,139.4$, 118.6 (q, $J=319.9 \mathrm{~Hz}), 67.4,48.8,31.6,25.8,24.0,20.8,20.4,20.3,18.2,-5.69,-5.74 ;{ }^{19} \mathrm{~F}$ NMR 
(471 MHz, $\mathrm{CDCl}_{3}$, internal standard $\mathrm{C}_{6} \mathrm{H}_{5} \mathrm{CF}_{3}$ ) $\delta$-75.1 (s); IR (ATR) $v_{\max }$ 2960, 2931, 2857, 1471, 1406, 1250, 1207, 1140, 1102, 1047, 1028, 1006, 971, 890, 775, 709, 670, 645, 612, $571 \mathrm{~cm}^{-1}$; HRMS (ESI) $[\mathrm{M}+\mathrm{Na}]^{+}$calculated for $\mathrm{C}_{17} \mathrm{H}_{31} \mathrm{~F}_{3} \mathrm{O}_{4} \mathrm{SSiNa}$; 439.1562, found, 439.1557; $[\alpha]_{\mathrm{D}}^{25}-23$ (c $2.6, \mathrm{MeOH})$.

(R)-5-hydroxymethyl-2-isopropyl-5-methylcyclopent-1-en-1-yl trifluoromethanesulfonate (14a)

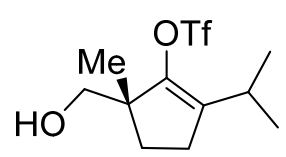

To a stirred solution of $14(10.8 \mathrm{~g}, 26.0 \mathrm{mmol})$ in $\mathrm{CH}_{2} \mathrm{Cl}_{2}(130 \mathrm{~mL})$ was added $3 \mathrm{HF} \cdot \mathrm{Et}_{3} \mathrm{~N}(26.0 \mathrm{~mL})$ at room temperature. The mixture was stirred at the same temperature for $47 \mathrm{~h}$ and quenched with saturated $\mathrm{NaHCO}_{3}$ solution at $0{ }^{\circ} \mathrm{C}$. The aqueous layer was extracted with $\mathrm{CH}_{2} \mathrm{Cl}_{2}(250 \mathrm{~mL} \times 3)$, and the combined organic layer was washed with brine $(150 \mathrm{~mL})$, dried over $\mathrm{Na}_{2} \mathrm{SO}_{4}$, filtered, and concentrated under reduced pressure. The residue was purified by flash column chromatography (hexane/ethyl acetate $=20 / 1)$ to afford $14 a(6.94 \mathrm{~g}, 88 \%)$ as a yellow clear oil: $\mathrm{R}_{\mathrm{f}}=0.65$ (hexane/ethyl acetate $\left.=2 / 1\right) ;{ }^{1} \mathrm{H}$ NMR $\left(500 \mathrm{MHz}, \mathrm{CDCl}_{3}\right) \delta 3.58(\mathrm{~d}, J$ $=11.5 \mathrm{~Hz}, 1 \mathrm{H}), 3.31(\mathrm{~d}, J=11.5 \mathrm{~Hz}, 1 \mathrm{H}), 2.84-2.92(\mathrm{~m}, 1 \mathrm{H}), 2.34-2.40(\mathrm{~m}, 1 \mathrm{H}), 2.24-2.30(\mathrm{~m}$, $1 \mathrm{H}), 2.17-2.22(\mathrm{~m}, 1 \mathrm{H}), 1.87(\mathrm{~s}, 1 \mathrm{H}), 1.67-1.72(\mathrm{~m}, 1 \mathrm{H}), 1.05(\mathrm{~s}, 3 \mathrm{H}), 1.04(\mathrm{~d}, J=6.5 \mathrm{~Hz}, 3 \mathrm{H})$, $1.04(\mathrm{~d}, J=6.5 \mathrm{~Hz}, 3 \mathrm{H}) ;{ }^{13} \mathrm{C} \mathrm{NMR}\left(125 \mathrm{MHz}, \mathrm{CDCl}_{3}\right) \delta 141.8,141.7,118.6(\mathrm{q}, J=318 \mathrm{~Hz}), 67.4$, 49.1, 31.6, 25.9, 24.2, 20.9, 20.5, 20.3; ${ }^{19} \mathrm{~F}$ NMR (471 MHz, $\mathrm{CDCl}_{3}$, internal standard $\mathrm{C}_{6} \mathrm{H}_{5} \mathrm{CF}_{3}$ ) $\delta-74.7$ (s); IR (ATR) $v_{\max } 3389,2968,2875,1682,1457,1404,1204,1137,1091,1045,1025$, 1002, 966, 886, 841, 768, 708, 645, 612, $571 \mathrm{~cm}^{-1}$; HRMS (ESI) $[\mathrm{M}+\mathrm{Na}]^{+}$calculated for $\mathrm{C}_{11} \mathrm{H}_{17} \mathrm{~F}_{3} \mathrm{O}_{4} \mathrm{SNa}$ : 325.0697, found 325.0692; $[\alpha]_{\mathrm{D}}^{25}-21$ (c 2.8, MeOH).

\section{(S)-5-formyl-2-isopropyl-5-methylcyclopent-1-en-1-yl trifluoromethanesulfonate (7)}

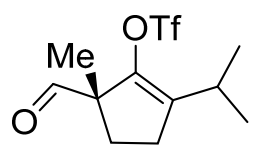

To a stirred solution of 14a $(6.94 \mathrm{~g}, 23.0 \mathrm{mmol})$ in $\mathrm{CH}_{2} \mathrm{Cl}_{2}(115 \mathrm{~mL})$ were added $\mathrm{NaHCO}_{3}(14.5 \mathrm{~g}, 17.2 \mathrm{mmol})$ and Dess-Martin periodinane $(21.4 \mathrm{~g}, 50.37 \mathrm{mmol})$ successively at $0{ }^{\circ} \mathrm{C}$. The mixture was stirred at room temperature for $50 \mathrm{~min}$ and quenched with saturated aqueous $\mathrm{NaHCO}_{3}$ solution $(100 \mathrm{~mL})$, and with saturated aqueous $\mathrm{Na}_{2} \mathrm{~S}_{2} \mathrm{O}_{3}$ solution $(100 \mathrm{~mL})$ at 
$0{ }^{\circ} \mathrm{C}$. The aqueous layer was extracted with $\mathrm{CH}_{2} \mathrm{Cl}_{2}(200 \mathrm{~mL} \times 3)$, and the combined organic layer was washed with brine $(100 \mathrm{~mL})$, dried over $\mathrm{Na}_{2} \mathrm{SO}_{4}$, and concentrated under reduced pressure. The residue was purified by flash column chromatography (hexane/ethyl acetate $=10 / 1$ ) to afford $7(6.16 \mathrm{~g}, 89 \%)$ as a clear orange oil: $\mathrm{R}_{\mathrm{f}}=0.28$ (hexane/ethyl acetate $\left.=10 / 1\right) ;{ }^{1} \mathrm{H}$ NMR $(500 \mathrm{MHz}$, $\left.\mathrm{CDCl}_{3}\right) \delta 9.56(\mathrm{~s}, 1 \mathrm{H}), 2.88-2.96(\mathrm{~m}, 1 \mathrm{H}), 2.38-2.48(\mathrm{~m}, 2 \mathrm{H}), 2.29-2.34(\mathrm{~m}, 1 \mathrm{H}), 1.78-1.84(\mathrm{~m}$, 1H), 1.29 (s, 3H), 1.08 (d, $J=7.0 \mathrm{~Hz}, 3 \mathrm{H}), 1.08(\mathrm{~d}, J=7.0 \mathrm{~Hz}, 3 \mathrm{H}) ;{ }^{13} \mathrm{C}$ NMR $\left(125 \mathrm{MHz}, \mathrm{CDCl}_{3}\right)$ $\delta 199.2,144.5,139.3,118.4(\mathrm{q}, J=318 \mathrm{~Hz}), 58.0,31.0,26.1,24.4,20.5,20.2,17.3 ;{ }^{19} \mathrm{~F}$ NMR (471 MHz, $\mathrm{CDCl}_{3}$, internal standard $\mathrm{C}_{6} \mathrm{H}_{5} \mathrm{CF}_{3}$ ) $\delta-74.8$ (s); IR (ATR) $v_{\max } 2971,1731,1458,1408$, 1206, 1136, 1093, 1050, 1030, 971, 890, 836, 768, 689, 606, $568 \mathrm{~cm}^{-1}$; HRMS (ESI) $[\mathrm{M}+\mathrm{Na}]^{+}$ calculated for $\mathrm{C}_{11} \mathrm{H}_{15} \mathrm{~F}_{3} \mathrm{O}_{4} \mathrm{SNa}$ : 323.0541, found: 323.0535; $[\alpha]_{\mathrm{D}}^{25}-32(c 1.8, \mathrm{MeOH})$.

\section{Enantioselective Total Synthesis of Cotylenol}

(5R)-5-((2S)-2-((R)-1-cyanoethyl)-5-oxocyclopentylhydroxymethyl)-2-isopropyl-5methylcyclopent-1-en-1-yl trifluoromethanesulfonate (17)

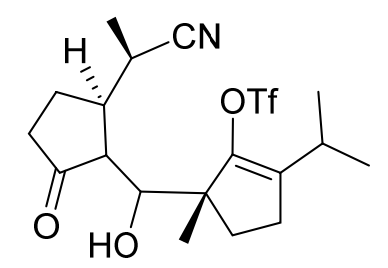

To a stirred solution of $\mathbf{1 6}(7.36 \mathrm{~g}, 27.0 \mathrm{mmol})$ and $\mathbf{7}(4.6 \mathrm{~g}, 15.33 \mathrm{mmol})$ in benzene $(90 \mathrm{~mL})$ were added $\mathrm{Ph}_{3} \mathrm{SnH}(9.46 \mathrm{~g}, 26.96 \mathrm{mmol})$ and $\mathrm{Et}_{3} \mathrm{~B}(0.99 \mathrm{M}$ in hexane, $27.3 \mathrm{~mL}, 27.0$ $\mathrm{mmol}$ ) at room temperature. The mixture was stirred at room temperature for $2 \mathrm{~h}$ and quenched with saturated aqueous KF solution $(100 \mathrm{~mL})$, and stirred for $30 \mathrm{~min}$. The resulting mixture was passed through a Celite pad and the aqueous layer was extracted with ethyl acetate $(100 \mathrm{~mL} \times 3)$. The combined organic layer was washed with brine $(100 \mathrm{~mL})$, dried over $\mathrm{Na}_{2} \mathrm{SO}_{4}$, filtered, and concentrated under reduced pressure. The residue was purified by flash column chromatography (hexane to hexane/ethyl acetate $=3 / 1$ ). The crude 17 (ca. $6.60 \mathrm{~g}$ including a small amount of impurities) was used for the next step without further purification: $\mathrm{R}_{\mathrm{f}}=0.50$ (hexane/ethyl acetate $=1 / 1) ;{ }^{1} \mathrm{H}$ NMR $\left(500 \mathrm{MHz}, \mathrm{CDCl}_{3}\right) \delta 3.66(\mathrm{~s}, 1 \mathrm{H}), 3.00-2.92(\mathrm{~m}, 1 \mathrm{H}), 2.92-2.78(\mathrm{~m}, 1 \mathrm{H}), 2.60$ $2.50(\mathrm{~m}, 1 \mathrm{H}), 2.50-2.15(\mathrm{~m}, 7 \mathrm{H}), 1.80-1.60(\mathrm{~m}, 2 \mathrm{H}), 1.43(\mathrm{~d}, J=7.5 \mathrm{~Hz}, 3 \mathrm{H}), 1.19(\mathrm{~s}, 3 \mathrm{H}), 1.03$ $(\mathrm{d}, J=3.0 \mathrm{~Hz}, 3 \mathrm{H}), 1.02(\mathrm{~d}, J=3.0 \mathrm{~Hz}, 3 \mathrm{H}) ;{ }^{13} \mathrm{C}\left(125 \mathrm{MHz}, \mathrm{CDCl}_{3}\right) \delta 215.2,142.4,141.0,120.2$, $76.8,73.1,53.5,52.0,44.5,38.5,28.8,28.6,25.8,24.2,22.6,22.1,20.4,20.2,16.2 ;{ }^{19} \mathrm{~F}$ NMR (471 MHz, $\mathrm{CDCl}_{3}$, internal standard $\mathrm{C}_{6} \mathrm{H}_{5} \mathrm{CF}_{3}$ ) $\delta-73.5$ (s); IR (ATR) $v_{\max } 3499,2969,1740,1457$, 
1403, 1206, 1136, 1045, 843, $604 \mathrm{~cm}^{-1}$; HRMS (ESI) $[\mathrm{M}+\mathrm{Na}]$ calcd for $\mathrm{C}_{19} \mathrm{H}_{26} \mathrm{O}_{5} \mathrm{NF}_{3} \mathrm{NaS}$ 460.1376, found 460.1375; $[\alpha]_{\mathrm{D}}^{25}-13\left(c 0.50, \mathrm{CHCl}_{3}\right)$.

\section{(R)-5-((E)-(S)-2-((R)-1-cyanoethyl)-5-oxocyclopentylidenemethyl)-2-isopropyl-5-}

\section{methylcyclopent-1-en-1-yl trifluoromethanesulfonate (5)}

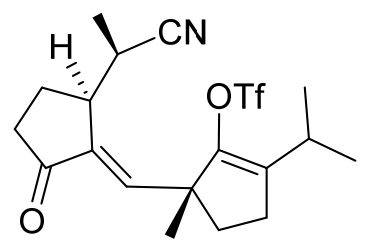

To a stirred solution of $\mathbf{1 7}(6.60 \mathrm{~g}, 15.1 \mathrm{mmol})$ in benzene $(151 \mathrm{~mL})$ was added Burgess reagent (14.4 g, $60.4 \mathrm{mmol})$ at room temperature. The mixture was stirred at room temperature for $12 \mathrm{~h}$, and then quenched with saturated aqueous $\mathrm{NaHCO}_{3}$ solution $(150 \mathrm{~mL})$. The aqueous layer was extracted with ethyl acetate $(100 \mathrm{~mL} \times 3)$, and the combined organic layer was washed with brine $(150 \mathrm{~mL})$, dried $\mathrm{Na}_{2} \mathrm{SO}_{4}$, filtered, and concentrated under reduced pressure. The residue was purified by flash column chromatography (hexane/ethyl acetate $=10 / 1)$ to afford $5(5.31 \mathrm{~g}$, $12.7 \mathrm{mmol}, 84 \%$ (2 steps)) as a colorless oil: $\mathrm{R}_{\mathrm{f}}=0.60$ (hexane/ethyl acetate $\left.=3 / 2\right) ;{ }^{1} \mathrm{H}$ NMR $(500$ MHz, $\left.\mathrm{CDCl}_{3}\right) \delta 6.64(\mathrm{~d}, J=1.5 \mathrm{~Hz}, 1 \mathrm{H}), 3.23(\mathrm{dd}, J=8.0,4.0 \mathrm{~Hz}, 1 \mathrm{H}), 2.87-2.75(\mathrm{~m}, 2 \mathrm{H}), 2.75-$ $2.62(\mathrm{~m}, 1 \mathrm{H}), 2.40-2.26(\mathrm{~m}, 2 \mathrm{H}), 2.35(\mathrm{~d}, J=8.0 \mathrm{~Hz}, 1 \mathrm{H}), 2.24-2.10(\mathrm{~m}, 2 \mathrm{H}), 2.05-1.90(\mathrm{~m}, 2 \mathrm{H})$, $1.42(\mathrm{~s}, 3 \mathrm{H}), 1.40(\mathrm{~d}, J=7.0 \mathrm{~Hz}, 3 \mathrm{H}), 1.04(\mathrm{~d}, J=7.0 \mathrm{~Hz}, 3 \mathrm{H}), 1.00(\mathrm{~d}, J=6.5 \mathrm{~Hz}, 3 \mathrm{H}) ;{ }^{13} \mathrm{C}(125$ $\left.\mathrm{MHz} \mathrm{CDCl}_{3}\right) \delta 205.9,143.0,141.8,140.1,137.6,121.3,118.4(\mathrm{q}, J=318 \mathrm{~Hz}), 77.4,48.7,40.8$, 37.5, 34.0, 30.8, 25.8, 24.0, 22.9, 21.8, 20.1, 17.1; ${ }^{19} \mathrm{~F}$ NMR (471 MHz, $\mathrm{CDCl}_{3}$, internal standard $\mathrm{C}_{6} \mathrm{H}_{5} \mathrm{CF}_{3}$ ) $\delta-74.6(\mathrm{~s})$; IR (ATR) $v_{\max } 2969,1754,1723,1632,1406,1206,1136,1046,1026,838$, $606 \mathrm{~cm}^{-1}$; HRMS (ESI) $[\mathrm{M}+\mathrm{Na}]$ calcd for $\mathrm{C}_{19} \mathrm{H}_{24} \mathrm{O}_{4} \mathrm{NF}_{3} \mathrm{NaS} 442.1270$, found $442.1271 ;[\alpha]_{\mathrm{D}}^{25}-$ 115 (c $\left.0.50, \mathrm{CHCl}_{3}\right)$.

\section{(R)-5-(E)-((S)-2-((R)-1-cyanoethyl)-5-oxocyclopentylidene)methyl-2-isopropyl-5-}

\section{methylcyclopent-1-en-1-yl trifluoromethanesulfonate (5a)}

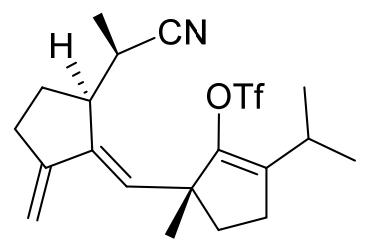

To a stirred suspension of $\mathrm{Zn}(17.3 \mathrm{~g}, 264 \mathrm{mmol})$ and $\mathrm{PbCl}_{2}(3.04 \mathrm{~g}, 10.9 \mathrm{mmol})$ in THF $(35.9 \mathrm{~mL}, 0.3 \mathrm{M})$ was added $\mathrm{CH}_{2} \mathrm{I}_{2}(6.91 \mathrm{~mL}, 86.2 \mathrm{mmol})$ at $0{ }^{\circ} \mathrm{C}$. The mixture was warmed 
up to room temperature and stirred for $1 \mathrm{~h}$. To the resultant mixture was added $\mathrm{ZrCl}_{4}(7.54 \mathrm{~g}, 32.3$ mmol) and the mixture was stirred at the same temperature. After $1 \mathrm{~h}$, a solution of ketone 5 $(4.52 \mathrm{~g}, 10.8 \mathrm{mmol})$ in THF $(21.6 \mathrm{~mL}, 0.5 \mathrm{M})$ was added to the stirred mixture at $0{ }^{\circ} \mathrm{C}$. After $1 \mathrm{~h}$, the reaction mixture was quenched with saturated aqueous $\mathrm{NaHCO}_{3}$ solution $(100 \mathrm{~mL})$. The resulting mixture was passed through a Celite pad and the aqueous layer was extracted with ethyl acetate $(150 \mathrm{~mL} \times 3)$. The combined organic layer was washed with brine $(300 \mathrm{~mL})$, dried over $\mathrm{Na}_{2} \mathrm{SO}_{4}$, filtered, and concentrated under reduced pressure. The crude $5 \mathrm{a}(8.33 \mathrm{~g})$ was used for the next reaction without further purification.

\section{$(R)-5-((E)-(2 R, 5 S)-5-((R)-1-c y a n o e t h y l)-2-h y d r o x y-2-$}

\section{hydroxymethylcyclopentylidenemethyl)-2-isopropyl-5-methylcyclopent-1-en-1-yl}

trifluoromethanesulfonate (18)

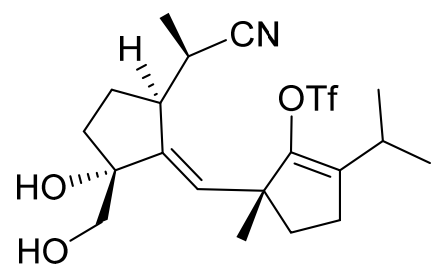

To a stirred solution of the crude $5 a(8.33 \mathrm{~g})$ in THF $(108 \mathrm{~mL})$ were added $\mathrm{H}_{2} \mathrm{O}(10.8$ $\mathrm{mL}), \mathrm{K}_{2} \mathrm{OsO}_{4} \cdot 2 \mathrm{H}_{2} \mathrm{O}(388.2 \mathrm{mg}, 1.08 \mathrm{mmol})$, and NMO (2.53 g, $\left.21.6 \mathrm{mmol}\right)$. The mixture was stirred at $0{ }^{\circ} \mathrm{C}$ for $38 \mathrm{~h}$, and then quenched with saturated aqueous $\mathrm{NaHCO}_{3}$ solution $(100 \mathrm{~mL})$, and with saturated aqueous $\mathrm{Na}_{2} \mathrm{~S}_{2} \mathrm{O}_{3}$ solution $(100 \mathrm{~mL})$. The resulting mixture was extracted with ethyl acetate $(200 \mathrm{~mL} \times 3)$. The combined organic layer was washed with brine $(300 \mathrm{~mL})$, dried over $\mathrm{Na}_{2} \mathrm{SO}_{4}$, filtered, and concentrated under reduced pressure. The residue was purified by flash column chromatography (hexane/ethyl acetate $=3 / 1)$ to afford $18(3.45 \mathrm{~g}, 7.64 \mathrm{mmol}, 71 \%(2$ steps)) as a colorless oil: $\mathrm{R}_{\mathrm{f}}=0.40$ (hexane/ethyl acetate $\left.=2 / 3\right) ;{ }^{1} \mathrm{H}$ NMR $\left(500 \mathrm{MHz}, \mathrm{CDCl}_{3}\right) \delta$ $5.86(\mathrm{~d}, J=2.5 \mathrm{~Hz}, 1 \mathrm{H}), 3.70(\mathrm{~d}, J=11.5 \mathrm{~Hz}, 1 \mathrm{H}), 3.58(\mathrm{~d}, J=11.5 \mathrm{~Hz}, 1 \mathrm{H}), 3.10-2.98(\mathrm{~m}, 2 \mathrm{H})$, 2.85-2.75 (m, 1H), 2.45-2.30 (m, 3H), 2.30-2.10 (m, 3H), 2.10-1.98 (m, 1H), 1.98-1.85 (m, 1H), 1.75-1.65 (m, 1H), 1.65-1.58 (m, 1H), 1.40 (s, 3H), 1.33 (d, $J=7.0 \mathrm{~Hz}, 3 \mathrm{H}), 1.04$ (d, $J=7.0 \mathrm{~Hz}$, $3 \mathrm{H}), 1.03(\mathrm{~d}, J=6.9 \mathrm{~Hz}, 3 \mathrm{H}) ;{ }^{13} \mathrm{C}\left(125 \mathrm{MHz}, \mathrm{CDCl}_{3}\right) \delta 146.9,145.6,138.0,132.7,121.8,118.2$ (q, $J=334 \mathrm{~Hz}), 82.3,67.5,48.0,42.7,36.7,34.5,30.3,25.6,24.7,24.0,23.6,20.2,20.1,17.0$; ${ }^{19} \mathrm{~F}$ NMR (471 MHz, $\mathrm{CDCl}_{3}$, internal standard $\left.\mathrm{C}_{6} \mathrm{H}_{5} \mathrm{CF}_{3}\right) \delta-74.9$ (s); IR (ATR) v $v_{\max } 3419,2967$, $2875,1456,1404,1207,1136,1028,968,840,755,605 \mathrm{~cm}^{-1}$; HRMS (ESI) [M+Na] calcd for $\mathrm{C}_{20} \mathrm{H}_{28} \mathrm{O}_{5} \mathrm{NF}_{3} \mathrm{NaS}$ 474.1532, found 474.1532; $[\alpha]_{\mathrm{D}}^{25}-49$ (c $\left.0.20, \mathrm{CHCl}_{3}\right)$. 
$(R)-5-((E)-((2 R, 5 S)-5-((R)-1-c y a n o e t h y l)-2-m e t h o x y m e t h y l-2-$

trimethylsilyloxycyclopentylidenemethyl)-2-isopropyl-5-methylcyclopent-1-en-1-yl

trifluoromethanesulfonate (18a)

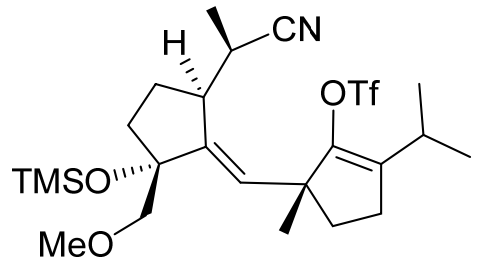

To a stirred solution of $\mathbf{1 8}(2.67 \mathrm{~g}, 5.91 \mathrm{mmol})$ in $\mathrm{CH}_{2} \mathrm{Cl}_{2}(59.1 \mathrm{~mL})$ were added 2,6di-tert-butyl-4-methylpyridine $(6.07 \mathrm{~g}, 29.5 \mathrm{mmol})$ and $\mathrm{Me}_{3} \mathrm{OBF}_{4}(2.19 \mathrm{~g}, 14.8 \mathrm{mmol})$ at $0{ }^{\circ} \mathrm{C}$. After the completion of methylation, pyridine $(2.4 \mathrm{~mL}, 29.5 \mathrm{mmol})$ and TMSCl (7.5 mL, 59.1 mmol) were added to the reaction mixture at $0{ }^{\circ} \mathrm{C}$. The mixture was stirred at $0{ }^{\circ} \mathrm{C}$ for $4 \mathrm{~h}$, and was quenched with saturated aqueous $\mathrm{NaHCO}_{3}$ solution $(100 \mathrm{~mL})$. The aqueous layer was extracted with ethyl acetate $(100 \mathrm{~mL} \times 3)$, and the combined organic layer was washed with brine $(200 \mathrm{~mL})$, dried $\mathrm{Na}_{2} \mathrm{SO}_{4}$, filtered, and concentrated under reduced pressure. The residue was purified by flash column chromatography (hexane/ethyl acetate $=20 / 1)$ to afford 18a $(2.23 \mathrm{~g}$, $4.15 \mathrm{mmol}, 71 \%$ ) as a white solid: $\mathrm{R}_{\mathrm{f}}=0.70$ (hexane/ethyl acetate $=5 / 1$ ); mp 49-50 ${ }^{\circ} \mathrm{C} ;{ }^{1} \mathrm{H}$ NMR $\left(500 \mathrm{MHz}, \mathrm{CDCl}_{3}\right) \delta 5.77(\mathrm{~d}, J=3.0 \mathrm{~Hz}, 1 \mathrm{H}), 3.48(\mathrm{~d}, J=10.0 \mathrm{~Hz}, 1 \mathrm{H}), 3.34(\mathrm{~s}, 3 \mathrm{H}), 3.25-3.12$ (m, 1H), $3.05(\mathrm{~d}, J=10.0 \mathrm{~Hz}, 1 \mathrm{H}), 2.96-2.88(\mathrm{~m}, 1 \mathrm{H}), 2.88-2.78(\mathrm{~m}, 1 \mathrm{H}), 2.43-2.25(\mathrm{~m}, 3 \mathrm{H})$, 2.24-2.10 (m, 1H), 2.05-1.85 (m, 2H), 1.70-1.53 (m, 2H), $1.36(\mathrm{~s}, 3 \mathrm{H}), 1.30$ (d, J = 6.5 Hz, 3H), $1.05(\mathrm{~d}, J=6.5 \mathrm{~Hz}, 3 \mathrm{H}), 1.04(\mathrm{~d}, J=6.5 \mathrm{~Hz}, 3 \mathrm{H}), 0.08(\mathrm{~s}, 9 \mathrm{H}) ;{ }^{13} \mathrm{C}\left(125 \mathrm{MHz}, \mathrm{CDCl}_{3}\right) \delta 146.7$, $145.9,137.3,132.0,121.9,118.5$ (q, $J=318 \mathrm{~Hz}), 84.4,77.1,58.9,47.4,42.2,36.5,33.5,29.7$, 25.6, 25.4, 23.97, 23.91, 20.14, 20.09. 17.2, 2.0; ${ }^{19} \mathrm{~F}$ NMR (471 MHz, $\mathrm{CDCl}_{3}$, internal standard $\left.\mathrm{C}_{6} \mathrm{H}_{5} \mathrm{CF}_{3}\right) \delta-74.8$ (s); IR (ATR) $v_{\max } 2965,1407,1248,1207,1138,1063,837,605 \mathrm{~cm}^{-1}$; HRMS (ESI) $[\mathrm{M}+\mathrm{Na}]$ calcd for $\mathrm{C}_{24} \mathrm{H}_{38} \mathrm{~F}_{3} \mathrm{NNaO}_{5} \mathrm{SSi} 560.2084$, found $560.2079 ;[\alpha]_{\mathrm{D}}^{25}-99$ (c 0.80 , $\mathrm{MeOH})$.

(R)-2-isopropyl-5-((E)-(2R,5S)-2-methoxymethyl)-5-((R)-1-oxopropan-2-yl)-2trimethylsilyloxycyclopentylidenemethyl-5-methylcyclopent-1-en-1-yl trifluoromethanesulfonate (19) 


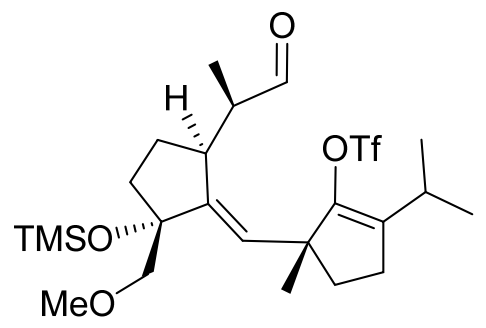

\section{$100 \mathrm{mg}$ scale prepration}

To a stirred solution of 18a $(100 \mathrm{mg}, 0.186 \mathrm{mmol})$ in $\mathrm{CH}_{2} \mathrm{Cl}_{2}(1.9 \mathrm{~mL})$ was added DIBAL-H (1.0 M in toluene, $0.22 \mathrm{~mL}, 0.22 \mathrm{mmol})$ at $-78{ }^{\circ} \mathrm{C}$. The mixture was stirred at $-78{ }^{\circ} \mathrm{C}$ for $1 \mathrm{~h}$, and quenched with saturated aqueous Rochelle salt solution $(10 \mathrm{~mL})$ at $0{ }^{\circ} \mathrm{C}$. The resulting mixture was passed through a Celite pad and the aqueous layer was extracted with ethyl acetate $(15 \mathrm{~mL} \times 3)$. The combined organic layer was washed with brine $(15 \mathrm{~mL})$, dried over $\mathrm{Na}_{2} \mathrm{SO}_{4}$, filtered, and concentrated under reduced pressure. The residue was purified by flash column chromatography (hexane/ethyl acetate $=10 / 1)$ to afford $19(96.3 \mathrm{mg}, 96 \%)$ as a colorless oil: $\mathrm{R}_{\mathrm{f}}$ $=0.70($ hexane/ethyl acetate $=4 / 1) ;{ }^{1} \mathrm{H}$ NMR $\left(500 \mathrm{MHz}, \mathrm{C}_{6} \mathrm{D}_{6}\right) \delta 9.72(\mathrm{~s}, 1 \mathrm{H}), 5.95-5.87(\mathrm{br}, 1 \mathrm{H})$, 3.19 (d, $J=10.0 \mathrm{~Hz}, 1 \mathrm{H}), 3.14$ (d, $J=10.0 \mathrm{~Hz}, 1 \mathrm{H}), 3.05$ (s, 3H), 3.00-2.85 (m, 2H), 2.80-2.70 (m, 1H), 2.15-1.85 (m, 4H), 1.75-1.48 (m, 3H), 1.39 (s, 3H), 1.38-1.32 (m, 1H), 0.93 (d, J = 7.0 $\mathrm{Hz}, 3 \mathrm{H}), 0.90$ (d, J=6.5 Hz, 3H), 0.79 (d, J=7.0 Hz, 3H), $0.21(\mathrm{~s}, 9 \mathrm{H}) ;{ }^{13} \mathrm{C}\left(125 \mathrm{MHz}, \mathrm{CDCl}_{3}\right)$ $\delta 203.7,148.1,146.5,137.4,130.9,119.3$ (q, $J=318 \mathrm{~Hz}$ ), 84.9, 78.9, 58.6, 48.9, 47.9, 42.0, 35.8, $35.1,26.0,25.5,25.0,23.9,20.08,19.94,12.8,2.4 ;{ }^{19} \mathrm{~F} \mathrm{NMR}\left(471 \mathrm{MHz}, \mathrm{CDCl}_{3}\right.$, internal standard $\left.\mathrm{C}_{6} \mathrm{H}_{5} \mathrm{CF}_{3}\right) \delta-74.9(\mathrm{~s})$; IR (ATR) $v_{\max } 2966,1722,1407,1248,1207,1137,836,605 \mathrm{~cm}^{-1}$; HRMS (ESI) $[\mathrm{M}+\mathrm{Na}]$ calcd for $\mathrm{C}_{24} \mathrm{H}_{39} \mathrm{~F}_{3} \mathrm{NaO}_{6} \mathrm{SSi} 563.2081$, found $563.2081 ;[\alpha]_{\mathrm{D}}^{25}-89$ (c 1.23, $\mathrm{CH}_{2} \mathrm{Cl}_{2}$ )

\section{Gram scale preparation}

To a stirred solution of 18a $(3.90 \mathrm{~g}, 7.10 \mathrm{mmol})$ in $\mathrm{CH}_{2} \mathrm{Cl}_{2}(71.0 \mathrm{~mL})$ was added DIBAL-H $(1.0 \mathrm{M}$ in toluene, $8.4 \mathrm{~mL}, 8.52 \mathrm{mmol})$ at $-78{ }^{\circ} \mathrm{C}$. The mixture was stirred at $-78{ }^{\circ} \mathrm{C}$ for one hour, and quenched with saturated aqueous Rochelle salt solution $(100 \mathrm{~mL})$ at $0{ }^{\circ} \mathrm{C}$. The resulting mixture was passed through a Celite pad and the aqueous layer was extracted with ethyl acetate $(150 \mathrm{~mL} \times 3)$. The combined organic layer was washed with brine $(150 \mathrm{~mL})$, dried over $\mathrm{Na}_{2} \mathrm{SO}_{4}$, filtered, and concentrated under reduced pressure. The residue was purified by flash column chromatography (hexane/ethyl acetate $=10 / 1)$ to afford $19(3.25 \mathrm{~g}, 85 \%)$ as a colorless oil. 
(5R)-5-((E)-(2R,5S)-5-((2R)-3-hydroxybutan-2-yl)-2-methoxymethyl-2-

\section{trimethylsilyloxycyclopentylidenemethyl)-2-isopropyl-5-methylcyclopent-1-en-1-yl \\ trifluoromethane sulfonate (19a)}

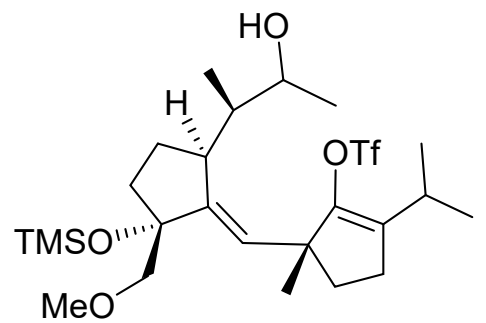

To a stirred solution of 19 (3.25 g, $6.01 \mathrm{mmol})$ was added $\mathrm{MeMgBr}(0.98 \mathrm{M}$ in THF, $6.7 \mathrm{~mL}, 6.61 \mathrm{mmol}$ ) at $-78{ }^{\circ} \mathrm{C}$. The reaction mixture was warmed up to $0{ }^{\circ} \mathrm{C}$ and stirred for 30 min, and quenched with saturated aqueous $\mathrm{NH}_{4} \mathrm{Cl}$ solution $(10 \mathrm{~mL})$. The aqueous layer was extracted with ethyl acetate $(10 \mathrm{~mL} \times 3)$, and the combined organic layer was washed with brine $(20 \mathrm{~mL})$, dried over $\mathrm{Na}_{2} \mathrm{SO}_{4}$, filtered, and concentrated under reduced pressure. The crude 19a (3.66 g) was used for the next step without further purification.

(R)-2-isopropyl-5-((E)-((2R,5S)-2-methoxymethyl)-5-((R)-3-oxobutan-2-yl)-2-

trimethylsilyloxycyclopentylidenemethyl)-5-methylcyclopent-1-en-1-yl

trifluoromethanesulfonate (4)

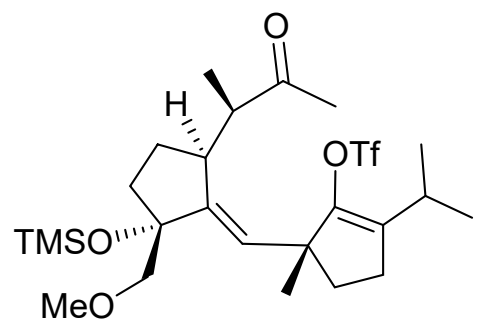

To a stirred solution of $19 \mathrm{a}(3.66 \mathrm{~g})$ in $\mathrm{CH}_{2} \mathrm{Cl}_{2}(60 \mathrm{~mL})$ were added $\mathrm{NaHCO}_{3}(3.53 \mathrm{~g}$, $42.1 \mathrm{mmol})$ and Dess-Martin periodinane $(5.10 \mathrm{~g}, 12.0 \mathrm{mmol})$ at room temperature. The reaction mixture was stirred at room temperature for $30 \mathrm{~min}$, and quenched with saturated aqueous $\mathrm{NaHCO}_{3}$ solution $(100 \mathrm{~mL})$, and with saturated aqueous $\mathrm{Na}_{2} \mathrm{~S}_{2} \mathrm{O}_{3}$ solution $(100 \mathrm{~mL})$. The aqueous layer was extracted with ethyl acetate $(100 \mathrm{~mL} \times 3)$, and the combined organic layer was washed with brine $(200 \mathrm{~mL})$, dried over $\mathrm{Na}_{2} \mathrm{SO}_{4}$, filtered, and concentrated under reduced pressure. The residue was purified by flash column chromatography (hexane/ethyl acetate $=10 / 1$ ) to afford 4 (3.03 g, 91\%, 2 steps) as a colorless oil: $\mathrm{R}_{\mathrm{f}}=0.40$ (hexane/ethyl acetate $\left.=3 / 1\right) ;{ }^{1} \mathrm{H}$ NMR $(500$ $\left.\mathrm{MHz}, \mathrm{C}_{6} \mathrm{D}_{6}\right) \delta 5.89(\mathrm{~d}, J=2.0 \mathrm{~Hz}, 1 \mathrm{H}), 3.38-3.30(\mathrm{br}, 1 \mathrm{H}), 3.22(\mathrm{~d}, J=2.0 \mathrm{~Hz}, 2 \mathrm{H}), 3.10(\mathrm{~s}, 3 \mathrm{H})$, 3.01-2.90 (m, 1H), 2.70-2.60 (m, 1H), 2.20-1.90 (m, 4H), 1.85 (s, 3H), 1.85-1.76 (m, 1H), 1.74- 
1.65 (m, 1H), 1.64-1.55 (m, 1H), 1.55-1.48 (m, 1H), 1.44 (s, 3H), 0.97 (d, J = 7.5 Hz, 3H), 0.92 $(\mathrm{d}, J=7.0 \mathrm{~Hz}, 3 \mathrm{H}), 0.82(\mathrm{~d}, J=7.0 \mathrm{~Hz}, 3 \mathrm{H}), 0.23(\mathrm{~s}, 9 \mathrm{H}) ;{ }^{13} \mathrm{C}\left(125 \mathrm{MHz}, \mathrm{CDCl}_{3}\right) \delta 209.4,148.2$, 146.6, 137.3, 130.8, 119.3 (q, $J=318 \mathrm{~Hz}), 84.7,79.7,58.7,48.8,48.7,42.0,36.5,34.1,30.1$, 27.5, 26.1, 24.9, 23.9, 20.2, 20.1, 16.4, 2.3; ${ }^{19} \mathrm{~F}$ NMR (471 MHz, $\mathrm{CDCl}_{3}$, internal standard $\left.\mathrm{C}_{6} \mathrm{H}_{5} \mathrm{CF}_{3}\right) \delta-74.8(\mathrm{~s})$; IR (ATR) $v_{\max } 2965,1712,1407,1247,1407,1207,1138,1107,837,606$ $\mathrm{cm}^{-1}$; HRMS (ESI) $[\mathrm{M}+\mathrm{Na}]$ calcd for $\mathrm{C}_{25} \mathrm{H}_{41} \mathrm{~F}_{3} \mathrm{NaO}_{6} \mathrm{SSi} 577.2237$, found 577.2236; $[\alpha]_{\mathrm{D}}^{25}-60(c$ $1.3, \mathrm{CH}_{2} \mathrm{Cl}_{2}$ ).

(1R,3aS,4R,9aR,E)-7-isopropyl-1-methoxymethyl-4,9a-dimethyl-1-trimethylsilyloxy2,3,3a,4,6,8,9,9a-octahydrodicyclopenta[a,d][8]annulen-5(1H)-one (3)

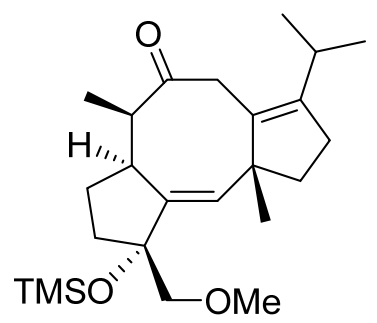

To a stirred solution of $\mathrm{PdCl}_{2}\left(\mathrm{PCy}_{3}\right)_{2}(146.3 \mathrm{mg}, 0.198 \mathrm{mmol})$ in toluene $(4.0 \mathrm{~mL})$ was added PhOK (209.6 mg, $1.59 \mathrm{mmol}$ ) at room temperature, and the resulting solution was warmed up to $110^{\circ} \mathrm{C}$. After $1 \mathrm{~h}$, the mixture was cooled to room temperature. To the prepared Pd solution was added $4(55.0 \mathrm{mg}, 0.0991 \mathrm{mmol})$ in toluene $(2.0 \mathrm{~mL})$ at room temperature. The resulting solution was warmed up to $50^{\circ} \mathrm{C}$. The reaction mixture was stirred at $50{ }^{\circ} \mathrm{C}$ for $8 \mathrm{~h}$, and quenched with saturated aqueous $\mathrm{NaHCO}_{3}$ solution $(10 \mathrm{~mL})$. The mixture was filtrated through a short Celite pad and the aqueous layer was extracted with ethyl acetate $(10 \mathrm{~mL} \times 3)$. The combined organic layer was washed with $1 \mathrm{M} \mathrm{NaOH}$ aq $(20 \mathrm{~mL})$, brine $(15 \mathrm{~mL})$, dried $\mathrm{Na}_{2} \mathrm{SO}_{4}$, filtered and concentrated under reduced pressure. The residue was purified by flash column chromatography (hexane/ethyl acetate $=20 / 1)$ to afford $3(38.0 \mathrm{mg}, 0.0938 \mathrm{mmol}, 95 \%)$ as a yellow oil: $\mathrm{R}_{\mathrm{f}}=0.65$ (hexane/ethyl acetate $=4 / 1)$; ${ }^{1} \mathrm{H}$ NMR $\left(500 \mathrm{MHz}, \mathrm{CDCl}_{3}\right) \delta 5.50(\mathrm{~s}, 1 \mathrm{H}), 3.40-3.30(\mathrm{br}, 1 \mathrm{H}), 3.35$ (s, 3H), 3.29-3.21 (br, 2H), 3.20 (d, $J=16.0 \mathrm{~Hz}, 1 \mathrm{H}), 3.10$ (d, $J=10.0 \mathrm{~Hz}, 1 \mathrm{H}), 2.99$ (d, $J=10.0$ $\mathrm{Hz}, 1 \mathrm{H}), 2.80(\mathrm{~d}, J=10.0 \mathrm{~Hz}, 1 \mathrm{H}), 2.75-2.65(\mathrm{~m}, 1 \mathrm{H}), 2.25$ (t, $J=6.5 \mathrm{~Hz}, 2 \mathrm{H}), 2.15-2.05$ (br, $1 \mathrm{H}), 1.90-1.70(\mathrm{~m}, 3 \mathrm{H}), 1.55-1.38(\mathrm{~m}, 2 \mathrm{H}), 1.35-0.80(\mathrm{~m}, 5 \mathrm{H}), 1.10(\mathrm{~s}, 3 \mathrm{H}), 1.13-0.95(\mathrm{~m}, 3 \mathrm{H})$, 0.07 (s, 9H); ${ }^{13} \mathrm{C}\left(125 \mathrm{MHz}, \mathrm{CDCl}_{3}\right) \delta 213.4,144.9,143.4,134.0,133.8,85.0,78.1,59.2,52.2$, $45.3,40.7,40.5,39.7,34.2,27.6,27.28,27.23,26.5,21.0,20.1,12.5,2.3$; IR (ATR) $v_{\max } 2956$, $1705,1451,1362,1246,1099,1020,904,836,752 \mathrm{~cm}^{-1}$; HRMS (ESI) [M+Na] calcd for $\mathrm{C}_{24} \mathrm{H}_{40} \mathrm{NaO}_{3} \mathrm{Si} 427.2639$, found 427.2638; $[\alpha]_{\mathrm{D}}^{25}-18$ (c 2.4, $\mathrm{CH}_{2} \mathrm{Cl}_{2}$ ). 
(1R,3aS,4S,9aR,E)-7-isopropyl-1-(methoxymethyl)-4,9a-dimethyl-1-((trimethylsilyl)oxy)2,3,3a,4,6,8,9,9a-octahydrodicyclopenta[a,d][8]annulen-5(1H)-one (3a)
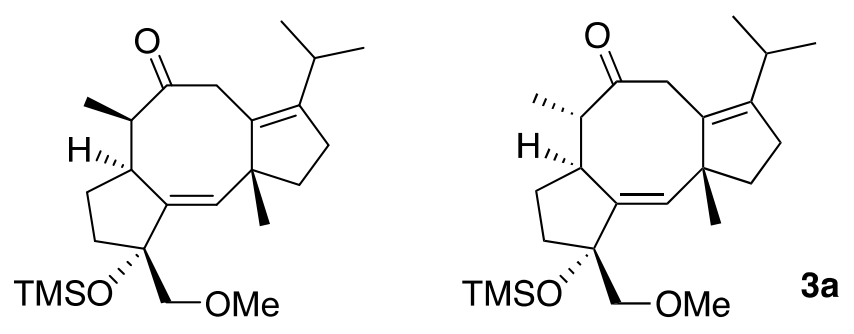

To a stirred solution of $4(185 \mathrm{mg}, 0.333 \mathrm{mmol})$ in toluene $(6.7 \mathrm{~mL})$ was added $\mathrm{PdCl}_{2}\left(\mathrm{PCy}_{3}\right)_{2}(123.1 \mathrm{mg}, 0.167 \mathrm{mmol})$ and $\mathrm{PhOK}(176.2 \mathrm{mg}, 1.33 \mathrm{mmol})$ at room temperature, and the resulting solution was warmed up to $100^{\circ} \mathrm{C}$. The reaction mixture was stirred at $100{ }^{\circ} \mathrm{C}$ for $3 \mathrm{~h}$ and quenched with saturated aqueous $\mathrm{NaHCO}_{3}$ solution $(10 \mathrm{~mL})$. The mixture was filtrated through a short Celite pad and the aqueous layer was extracted with ethyl acetate $(10 \mathrm{~mL} \times 3)$. The combined organic layer was washed with $1 \mathrm{M} \mathrm{NaOH}$ aq $(20 \mathrm{~mL})$, brine $(15 \mathrm{~mL})$, dried $\mathrm{Na}_{2} \mathrm{SO}_{4}$, filtered and concentrated under reduced pressure. The residue was purified by flash column chromatography (hexane/ethyl acetate $=20 / 1)$ to afford 3 and $3 a(117.1 \mathrm{mg}, 0.289 \mathrm{mmol}, 87 \%)$ as a yellow oil $(\mathbf{3}: \mathbf{3 a}=1.8: 1): \mathrm{R}_{\mathrm{f}}=0.65$ (hexane/ethyl acetate $\left.=4 / 1\right)$.

3a: $\mathrm{R}_{\mathrm{f}}=0.65$ (hexane/ethyl acetate $\left.=4 / 1\right) ; \mathrm{mp} 59-61{ }^{\circ} \mathrm{C} ;{ }^{1} \mathrm{H}$ NMR $\left(500 \mathrm{MHz}, \mathrm{CDCl}_{3}\right) \delta 5.40(\mathrm{~d}$, $J=1.7 \mathrm{~Hz}, 1 \mathrm{H}), 3.65$ (d, $J=14.9 \mathrm{~Hz}, 1 \mathrm{H}), 3.34$ (s, 3H), 3.25-3.19 (br, 1H), 3.13 (d, $J=10.3 \mathrm{~Hz}$, $1 \mathrm{H}), 3.10(\mathrm{~d}, J=10.3 \mathrm{~Hz}, 1 \mathrm{H}), 2.82-2.72(\mathrm{~m}, 2 \mathrm{H}), 2.23-2.14(\mathrm{~m}, 3 \mathrm{H}), 2.04-1.99(\mathrm{~m}, 1 \mathrm{H}), 1.92-$ $1.88(\mathrm{~m}, 1 \mathrm{H}), 1.84-1.78(\mathrm{~m}, 1 \mathrm{H}), 1.72-1.66(\mathrm{~m}, 1 \mathrm{H}), 1.60-1.54(\mathrm{~m}, 1 \mathrm{H}), 1.26-1.21(\mathrm{~m}, 1 \mathrm{H}), 1.16$ (d, $J=6.9 \mathrm{~Hz}, 3 \mathrm{H}), 1.12$ (s, 3H), 1.08 (d, $J=6.0 \mathrm{~Hz}, 3 \mathrm{H}), 0.99$ (d, $J=6.3 \mathrm{~Hz}, 3 \mathrm{H}), 0.07$ (s, 9H); ${ }^{13} \mathrm{C}\left(125 \mathrm{MHz}, \mathrm{CDCl}_{3}\right) \delta 213.3,144.0(\mathrm{x} 2), 132.7,132.3,84.0,79.1,59.2,56.0,52.8,41.8,39.8$, 38.1, 34.1, 28.2, 27.6, 27.4, 21.5, 20.0, 16.7, 2.3; IR (ATR) $v_{\max } 2958,2871,1704,1457,1248$, $1165,1035,861,839 \mathrm{~cm}^{-1}$; HRMS (ESI) $[\mathrm{M}+\mathrm{Na}]$ calcd for $\mathrm{C}_{24} \mathrm{H}_{40} \mathrm{NaO}_{3} \mathrm{Si} 427.2634$, found 427.2639; $[\alpha]_{\mathrm{D}}^{25}+134\left(\right.$ c $\left.1.5, \mathrm{CH}_{2} \mathrm{Cl}_{2}\right)$.

(1R,3aS,4R,6R,9aR,E)-6-hydroxy-7-isopropyl-1-methoxymethyl-4,9a-dimethyl-1trimethylsilyloxy-2,3,3a,4,6,8,9,9a-octahydrodicyclopenta[a,d][8]annulen-5(1H)-one (20) 


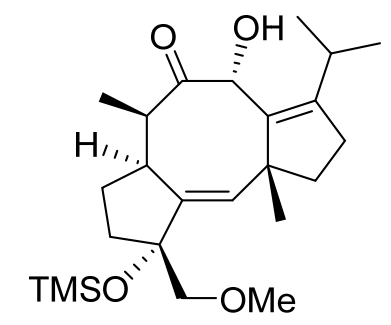

To a stirred solution of $3(275.2 \mathrm{mg}, 0.680 \mathrm{mmol})$ and $\mathrm{LiCl}(288.0 \mathrm{mg}, 6.80 \mathrm{mmol})$ in THF $(13.6 \mathrm{~mL})$ was added LHMDS $(1.12 \mathrm{M}$ in THF, $1.82 \mathrm{~mL}, 2.04 \mathrm{mmol})$ at $-50{ }^{\circ} \mathrm{C}$. After the completion of addition, the reaction mixture was gradually warmed up to $0{ }^{\circ} \mathrm{C}$. After $1 \mathrm{~h}$, to the reaction mixture cooled at $-60{ }^{\circ} \mathrm{C}$ was added $\mathrm{MoOPH}(1.18 \mathrm{~g}, 2.72 \mathrm{mmol})$. The reaction mixture was stirred at $-40{ }^{\circ} \mathrm{C}$ for $3 \mathrm{~h}$, and then quenched with saturated aqueous $\mathrm{NaHCO}_{3}$ solution $(20$ $\mathrm{mL}$ ) and then, saturated aqueous $\mathrm{Na}_{2} \mathrm{~S}_{2} \mathrm{O}_{3}$ solution $(20 \mathrm{~mL})$. The aqueous layer was extracted with ethyl acetate $(20 \mathrm{~mL} \times 3)$, and the combined organic layer was washed with brine $(50 \mathrm{~mL})$, dried over $\mathrm{Na}_{2} \mathrm{SO}_{4}$, filtered, and concentrated under reduced pressure. The residue was purified by flash column chromatography (hexane/ethyl acetate $=10 / 1)$ to afford $20(148.5 \mathrm{mg}, 0.353 \mathrm{mmol}, 52 \%)$ and C9 epimer 20a (55.0 mg, $0.131 \mathrm{mmol}, 19 \%)$ as a colorless oil:

20: $\mathrm{R}_{\mathrm{f}}=0.60$ (hexane/ethyl acetate $\left.=3 / 1\right) ;{ }^{1} \mathrm{H} \mathrm{NMR}\left(500 \mathrm{MHz}, \mathrm{CDCl}_{3}\right) \delta 5.63(\mathrm{~d}, J=2.0 \mathrm{~Hz}, 1 \mathrm{H})$, 4.81 (s, 1H), 3.80 (br, 1H), 3.36 (s, 3H), 3.31 (d, $J=10.0 \mathrm{~Hz}, 1 \mathrm{H}), 3.36-3.19$ (m, 1H), 3.06 (d, $J$ $=10.0 \mathrm{~Hz}, 1 \mathrm{H}), 2.80-2.70(\mathrm{~m}, 1 \mathrm{H}), 2.70-2.63(\mathrm{~m}, 1 \mathrm{H}), 2.23-2.11(\mathrm{~m}, 2 \mathrm{H}), 2.12-2.05(\mathrm{~m}, 1 \mathrm{H})$, 2.00-1.90 (m, 1H), 1.85-1.75 (m, 2H), 1.53-1.42 (m, 1H), 1.38-1.22 (m, 3H), $1.31(\mathrm{~s}, 3 \mathrm{H}), 1.15-$ $1.10(\mathrm{~m}, 2 \mathrm{H}), 1.08$ (d, J=7.0 Hz, 3H), 1.00 (d, $J=6.5 \mathrm{~Hz}, 3 \mathrm{H}), 0.81$ (d, J=7.0 Hz, 3H), 0.09 (s, $9 \mathrm{H}) ;{ }^{13} \mathrm{C}\left(125 \mathrm{MHz}, \mathrm{CDCl}_{3}\right) \delta 212.0,150.8,139.7,135.5,133.2,85.1,77.5,69.4,59.2,52.5,50.1$, $40.9,39.1,34.2,30.2,27.6,27.0,26.3,21.0,19.6,13.4,2.3$; IR (ATR) $v_{\max } 3473,2956,2361$, $1698,1451,1247,1149,1104,1053,839 \mathrm{~cm}^{-1}$; HRMS (ESI) [M+Na] calcd for $\mathrm{C}_{24} \mathrm{H}_{40} \mathrm{NaO}_{4} \mathrm{Si}$ 443.2588, found 443.2587; $[\alpha]_{\mathrm{D}}^{25}-9(c 0.50, \mathrm{MeOH})$.

20a: $\mathrm{R}_{\mathrm{f}}=0.62$ (hexane/ethyl acetate $\left.=3 / 1\right) ;{ }^{1} \mathrm{H}$ NMR $\left(500 \mathrm{MHz}, \mathrm{CDCl}_{3}\right) \delta 5.42(\mathrm{~d}, J=2.0 \mathrm{~Hz}$, 1H), 5.09 (d, $J=3.5 \mathrm{~Hz}, 1 \mathrm{H}), 3.90(\mathrm{~d}, \mathrm{~J}=4.0 \mathrm{~Hz}, 1 \mathrm{H}), 3.76$ (br, $1 \mathrm{H}), 3.35$ (s, 3H), 3.23 (d, $J=10$ $\mathrm{Hz}, 1 \mathrm{H}), 3.15(\mathrm{~d}, J=10 \mathrm{~Hz}, 1 \mathrm{H}), 3.05-2.95(\mathrm{~m}, 1 \mathrm{H}), 2.88-2.80(\mathrm{~m}, 1 \mathrm{H}), 2.30-2.15(\mathrm{~m}, 2 \mathrm{H})$, 2.08-1.96 (m, 1H), 1.96-1.85 (m, 1H), 1.86-1.78 (m, 1H), 1.78-1.68 (m, 1H), 1.65-1.55 (m, 2H), $1.11(\mathrm{~d}, J=6.5 \mathrm{~Hz}, 3 \mathrm{H}), 1.10$ (s, 3H), 1.051 (d, $J=7.0 \mathrm{~Hz}, 3 \mathrm{H}), 1.046$ (d, $J=7.0 \mathrm{~Hz}, 3 \mathrm{H}), 0.06$ (s, 9H); ${ }^{13} \mathrm{C}\left(125 \mathrm{MHz}, \mathrm{CDCl}_{3}\right) \delta 213.3,151.0,143.6,135.3,134.6,84.8,78.1,72.8,59.2,52.6$, $47.4,41.8,38.3,35.6,28.0,27.5,27.3,27.0,21.6,20.5,10.8,2.3(x 3)$; IR (ATR) $v_{\max } 3467,2955$, $1702,1453,1378,1246,1104,836,752,685 \mathrm{~cm}^{-1}$; HRMS (ESI) $[\mathrm{M}+\mathrm{Na}]$ calcd for $\mathrm{C}_{24} \mathrm{H}_{40} \mathrm{NaO}_{4} \mathrm{Si}$ 443.2588, found 443.2586; $[\alpha]_{\mathrm{D}}^{25}+73$ (c 2.8, MeOH). 
(4R,5R,6R,6aS,9R,10aR,E)-3-isopropyl-9-methoxymethyl-6,10a-dimethyl-9trimethylsilyloxy-1,2,4,5,6,6a,7,8,9,10a-decahydrodicyclopenta[a,d][8]annulene-4,5-diol (21)

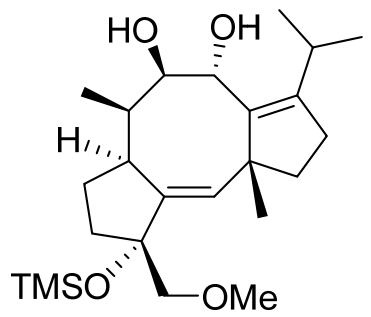

\section{Preparation of 1.0 M Me $4{ }_{4} \mathrm{NBH}\left(\mathrm{O}_{2} \mathrm{C}^{i} \mathrm{Pr}\right)_{3}$ in $\mathrm{CH}_{3} \mathrm{CN}$ solution}

To a stirred solution of $\mathrm{Me}_{4} \mathrm{NBH}_{4}(3.05 \mathrm{~g}, 30.8 \mathrm{mmol})$ in $\mathrm{CH}_{3} \mathrm{CN}(21 \mathrm{~mL})$ was added isobutyric acid $(10 \mathrm{~mL}, 107.8 \mathrm{mmol})$ at $0{ }^{\circ} \mathrm{C}$. After $30 \mathrm{~min}$, the reaction mixture was gradually warmed up to room temperature. After $4 \mathrm{~h}$, the resulting solution was used for the reaction of $\mathbf{2 0}$.

To a stirred solution of $20(23.0 \mathrm{mg}, 0.0546 \mathrm{mmol})$ in $\mathrm{CH}_{3} \mathrm{CN}(0.1 \mathrm{~mL})$ was added $\mathrm{Me}_{4} \mathrm{NBH}\left(\mathrm{O}_{2} \mathrm{C}^{i} \mathrm{Pr}\right)_{3}\left(1.0 \mathrm{M}\right.$ in $\left.\mathrm{CH}_{3} \mathrm{CN}, 2.1 \mathrm{~mL}, 2.18 \mathrm{mmol}\right)$ at room temperature. The reaction mixture was stirred at room temperature for $24 \mathrm{~h}$, and quenched with saturated aqueous $\mathrm{NaHCO}_{3}$ solution $(20 \mathrm{~mL})$, and with $5 \mathrm{M}$ aqueous trimethylolethane solution $(20 \mathrm{~mL})$. The mixture was stirred at room temperature for $12 \mathrm{~h}$, and the aqueous layer was extracted with ethyl acetate (10 $\mathrm{mL} \times 3)$. The combined organic layer was washed with brine $(15 \mathrm{~mL})$, dried over $\mathrm{Na}_{2} \mathrm{SO}_{4}$, filtered, and concentrated under reduced pressure. The residue was purified by flash column chromatography (hexane/ethyl acetate $=10 / 1)$ to afford $21(18.5 \mathrm{mg}, 80 \%)$ as colorless oil: $\mathrm{R}_{\mathrm{f}}=$ 0.45 (hexane/ethyl acetate $=3 / 1) ;{ }^{1} \mathrm{H}$ NMR $\left(500 \mathrm{MHz}, \mathrm{C}_{6} \mathrm{D}_{6}\right) \delta 5.64(\mathrm{~d}, J=2.0 \mathrm{~Hz}, 1 \mathrm{H}), 4.03(\mathrm{~d}$, $J=9.5 \mathrm{~Hz}, 1 \mathrm{H}), 3.91(\mathrm{dd}, J=9.5,4.0 \mathrm{~Hz}, 1 \mathrm{H}), 3.45-3.30(\mathrm{~m}, 1 \mathrm{H}), 3.30(\mathrm{~d}, J=10.0 \mathrm{~Hz}, 1 \mathrm{H}), 3.25$ (s, 3H), 2.99 (d, $J=10.5 \mathrm{~Hz}, 1 \mathrm{H}), 2.89$ (t, $J=9.0 \mathrm{~Hz}, 1 \mathrm{H}), 2,68$ (br, 1H), 2.25 (dd, $J=12.0,6.0$ $\mathrm{Hz}, 1 \mathrm{H}), 2.23-2.12(\mathrm{~m}, 1 \mathrm{H}), 2.01$ (dd, $J=15.5,8.0 \mathrm{~Hz}, 1 \mathrm{H}), 2.00-1.88(\mathrm{~m}, 1 \mathrm{H}), 1.80-1.60$ (m, 2H), 1.55-1.20 (m, 3H), 1.22-1.08 (m, 1H), $1.17(\mathrm{~s}, 3 \mathrm{H}), 1.02(\mathrm{~d}, J=7.0 \mathrm{~Hz}, 3 \mathrm{H}), 0.93(\mathrm{~d}, J=$ $7.0 \mathrm{~Hz}, 3 \mathrm{H}), 0.89(\mathrm{~d}, J=7.0 \mathrm{~Hz}, 3 \mathrm{H}), 0.26(\mathrm{br}, 9 \mathrm{H}) ;{ }^{13} \mathrm{C}\left(125 \mathrm{MHz}, \mathrm{C}_{6} \mathrm{D}_{6}\right) \delta 149.8,140.7,137.3$, 134.0, 85.6, 78.1, 77.5, 68.1, 58.9, 51.9, 43.4, 41.8, 40.3, 34.4, 31.5, 28.3, 27.3, 26.6, 21.5, 20.5, 8.5, 2.6; IR (ATR) $v_{\max } 3420,2954,2870,1452,1404,1312,1248,1103,1054,990,838,754 \mathrm{~cm}^{-}$ ${ }^{1}$; HRMS (ESI) $[\mathrm{M}+\mathrm{Na}]$ calcd for $\mathrm{C}_{24} \mathrm{H}_{42} \mathrm{NaO}_{4} \mathrm{Si} 445.2725$, found 445.2728; [ $\left.\alpha\right]_{\mathrm{D}}^{25}-40$ (c 0.20, $\mathrm{MeOH})$. 


\section{Cotylenol}

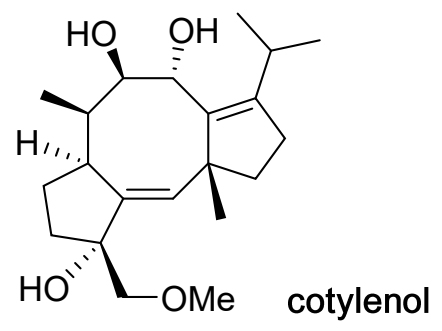

To a stirred solution of $21(1.3 \mathrm{mg}, 3.07 \mu \mathrm{mol})$ in THF $(0.47 \mathrm{~mL})$ was added TBAF $(1.0 \mathrm{M}$ in $\mathrm{THF}, 24 \mu \mathrm{L}, 23.6 \mu \mathrm{mol})$ at room temperature. The reaction mixture was stirred at room temperature for $2.5 \mathrm{~h}$, and was quenched with saturated aqueous $\mathrm{NH}_{4} \mathrm{Cl}$ solution $(10 \mathrm{~mL})$. The aqueous layer was extracted with ethyl acetate $(5 \mathrm{~mL} \times 3)$, and the combined organic layer was washed with brine $(10 \mathrm{~mL})$, dried over $\mathrm{Na}_{2} \mathrm{SO}_{4}$, filtered, and concentrated under reduced pressure. The residue was purified by flash column chromatography (hexane/ethyl acetate $=4 / 1$ ) to afford cotylenol (1.0 mg, $2.86 \mu \mathrm{mol}, 93 \%)$ as a white solid. $\mathrm{R}_{\mathrm{f}}=0.50$ (hexane/ethyl acetate $\left.=1 / 1\right) ; \mathrm{mp}$ $155-158^{\circ} \mathrm{C} ;{ }^{1} \mathrm{H}$ NMR $\left(600 \mathrm{MHz}, \mathrm{CDCl}_{3}\right) \delta 5.52(\mathrm{~d}, J=2.6 \mathrm{~Hz}, 1 \mathrm{H}), 4.07(\mathrm{~d}, J=10.2 \mathrm{~Hz}, 1 \mathrm{H})$, $3.94(\mathrm{dd}, J=10.2,4.4 \mathrm{~Hz}, 1 \mathrm{H}), 3.40(\mathrm{~s}, 3 \mathrm{H}), 3.36$ (d, $J=9.7 \mathrm{~Hz}, 1 \mathrm{H}), 3.29-3.25$ (m, 1H), 3.08 (dd, $J=9.5,1.3 \mathrm{~Hz}, 1 \mathrm{H}), 2.95-2.92(\mathrm{~m}, 2 \mathrm{H}), 2.52(\mathrm{br}, 1 \mathrm{H}), 2.16-2.08(\mathrm{~m}, 2 \mathrm{H}), 2.03-1.93(\mathrm{~m}$, $3 \mathrm{H}), 1.85(\mathrm{ddd}, J=11.9,6.8,2.0 \mathrm{~Hz}, 1 \mathrm{H}), 1.76(\mathrm{~s}, 1 \mathrm{H}), 1.71-1.66(\mathrm{~m}, 1 \mathrm{H}), 1.43-1.40(\mathrm{~m}, 1 \mathrm{H})$, 1.30-1.24 (m, 1H), $1.22(\mathrm{~s}, 3 \mathrm{H}), 1.04(\mathrm{~d}, J=6.9 \mathrm{~Hz}, 3 \mathrm{H}), 0.96(\mathrm{~d}, J=6.9 \mathrm{~Hz}, 3 \mathrm{H}), 0.81$ (d, $J=$ $7.2 \mathrm{~Hz}, 3 \mathrm{H}) ;{ }^{13} \mathrm{C}\left(150 \mathrm{MHz}, \mathrm{CDCl}_{3}\right) \delta 150.4,139.7,136.9,134.2,81.9,77.5,77.3,67.8,59.3$, $51.8,42.5,41.6,40.2,35.3,31.6,28.0,27.1,26.5,21.4,20.3,8.4$

${ }^{1} \mathrm{H}$ NMR (500 MHz, $\left.\mathrm{C}_{6} \mathrm{D}_{6}\right) \delta ; 5.74-5.71(\mathrm{~m}, 1 \mathrm{H}), 4.02(\mathrm{~d}, J=10.5 \mathrm{~Hz}, 1 \mathrm{H}), 3.93(\mathrm{dd}$, $J=10.5,4.0 \mathrm{~Hz}, 1 \mathrm{H}), 3.41-3.31(\mathrm{~m}, 1 \mathrm{H}), 3.28$ (d, $J=9.0 \mathrm{~Hz}, 1 \mathrm{H}), 3.06(\mathrm{~s}, 3 \mathrm{H}), 3.05-2.96(\mathrm{~m}$, 1H), 2.71 (br, 1H), 2.16-2.07 (m, 1H), 2.07-2.01 (m, 1H), 2.00-1.95 (m, 3H), 1.88-1.77 (m, 2H), $1.68-1.57(\mathrm{~m}, 1 \mathrm{H}), 1.57-1.45(\mathrm{~m}, 1 \mathrm{H}), 1.40-1.20(\mathrm{~m}, 2 \mathrm{H}), 1.16(\mathrm{~s}, 3 \mathrm{H}), 1.13-1.05(\mathrm{~m}, 1 \mathrm{H}), 1.00$ $(\mathrm{d}, J=6.0 \mathrm{~Hz}, 3 \mathrm{H}), 0.91(\mathrm{~d}, \mathrm{~J}=7.5 \mathrm{~Hz}, 3 \mathrm{H}), 0.89(\mathrm{~d}, J=6.0 \mathrm{~Hz}, 3 \mathrm{H}) ;{ }^{13} \mathrm{C}\left(125 \mathrm{MHz}, \mathrm{C}_{6} \mathrm{D}_{6}\right) \delta$ 149.9, 139.9, 137.5, 134.4, 81.9, 77.96, 77.93, 68.1, 58.9, 52.0, 43.0, 42.2 , 40.6, 35.7, 31.8, 28.3, 27.3, 26.7, 21.5, 20.4, 8.7; IR (ATR) $\mathrm{v}_{\max } 3408,2953,2926,2867,1723,1452,1359,1298,1100$, 1039, 957, $759 \mathrm{~cm}^{1}{ }^{1}$; HRMS (ESI+) $[\mathrm{M}+\mathrm{Na}]$ calcd for $\mathrm{C}_{21} \mathrm{H}_{34} \mathrm{NaO}_{4} 373.2349$, found 373.2347; $[\alpha]_{\mathrm{D}}^{25}-29(c 0.18, \mathrm{MeOH})$. 


\section{Preparation of Sugar Part Fragment}

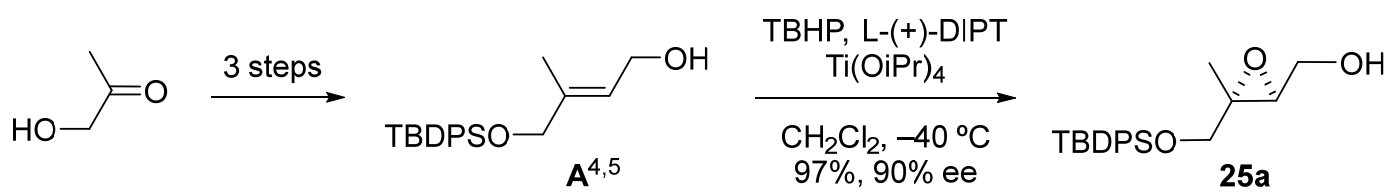

$\begin{array}{r}\text { 1) } \mathrm{TsCl} \text {, imidazole } \\ \mathrm{CH}_{2} \mathrm{Cl}_{2} \mathrm{rt}, 72 \% \\ \hline\end{array}$

2) TBAF, THF, rt, $84 \%$

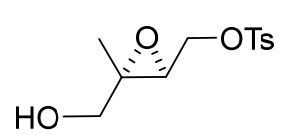

25c

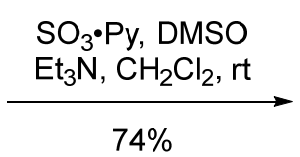

$74 \%$

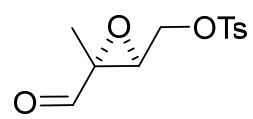

25

\section{(2S,3S)-3-tert-butyldiphenylsilyloxymethyl-3-methyloxiran-2-ylmethanol (25a)}

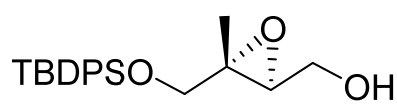

To a stirred suspension of activated powdered molecular sieves $4 \AA(15.0 \mathrm{~g})$ in $\mathrm{CH}_{2} \mathrm{Cl}_{2}$ $(200 \mathrm{~mL})$ were added a solution of $(L)$-(+)-diisopropyl tartrate $(10.4 \mathrm{~g}, 44.5 \mathrm{mmol})$ in $\mathrm{CH}_{2} \mathrm{Cl}_{2}(50$ $\mathrm{mL})$ and $\mathrm{Ti}(\mathrm{OiPr})_{4}(10.9 \mathrm{~mL}, 36.8 \mathrm{mmol})$ at room temperature. Then, a solution of tert-butyl hydroperoxide (3.0 $\mathrm{M}_{\text {in }} \mathrm{CH}_{2} \mathrm{Cl}_{2}, 98.9 \mathrm{~mL}, 297 \mathrm{mmol}$ ) was added dropwise to the reaction mixture over $10 \mathrm{~min}$ and the resultant mixture was stirred at $-20{ }^{\circ} \mathrm{C}$ for $30 \mathrm{~min}$. Then a solution of allylic alcohol $\mathrm{A}^{4,5}$ (50.5 g, $\left.148 \mathrm{mmol}\right)$ in $\mathrm{CH}_{2} \mathrm{Cl}_{2}(100 \mathrm{~mL})$ was added dropwise to the reaction mixture over 10 min with vigorous stirring. After stirring at $-20{ }^{\circ} \mathrm{C}$ for $12 \mathrm{~h}$, the reaction mixture was warmed up to room temperature and to the mixture was added saturated aqueous $\mathrm{Na}_{2} \mathrm{SO}_{4}(100$ $\mathrm{mL}$ ). After stirring for $3 \mathrm{~h}$, the resulting mixture was filtered through a Celite pad, and the filtrate was concentrated in vacuo. The residue was purified by flash column chromatography (hexane/ethyl acetate $=20 / 1)$ to afford 25a $(52.0 \mathrm{~g}, 146 \mathrm{mmol}, 97 \%, 88 \%$ ee) as a colorless oil. The enantiomeric excess was determined by HPLC analysis with a chiral HPLC column (DAICEL CHIRALCEL IC-3, 9\% 2-propanol in hexane, $1.0 \mathrm{~mL} / \mathrm{min}$ ). The retention times for 25a and its enantiomer were 8.88 and $7.13 \mathrm{~min}$, respectively: $\mathrm{R}_{\mathrm{f}}=0.32$ (hexane/ethyl acetate $=$ 2/1); ${ }^{1} \mathrm{H}$ NMR (500 MHz, $\left.\mathrm{CDCl}_{3}\right) \delta 7.68-7.65$ (m, 4H), 7.45-7.37 (m, 6H), 3.83 (dd, $J=12.0$, $4.0 \mathrm{~Hz}, 1 \mathrm{H}), 3.69(\mathrm{dd}, J=12.0,7.0 \mathrm{~Hz}, 1 \mathrm{H}), 3.66(\mathrm{~d}, J=11.5 \mathrm{~Hz}, 1 \mathrm{H}), 3.63(\mathrm{~d}, J=11.5 \mathrm{~Hz}, 1 \mathrm{H})$, $3.08(\mathrm{dd}, J=7.0,4.0 \mathrm{~Hz}, 1 \mathrm{H}), 1.34(\mathrm{~s}, 3 \mathrm{H}), 1.05(\mathrm{~s}, 9 \mathrm{H}) ;{ }^{13} \mathrm{C} \mathrm{NMR}\left(125 \mathrm{MHz}, \mathrm{CDCl}_{3}\right) \delta 135.65$, 135.57, 133.2, 129.8, 127.74, 127.72, 67.6, 61.2, 60.2, 26.8, 19.3, 14.4; IR (ATR) $v_{\max } 3444,2931$, 2857, 1739, 1472, 1427, 1376, 1265, 1104, 1028, 938, 901, 864 822, 740, 700, $613 \mathrm{~cm}^{-1}$; HRMS (ESI): $[\mathrm{M}+\mathrm{Na}]^{+}$calcd for $\mathrm{C}_{21} \mathrm{H}_{28} \mathrm{O}_{3} \mathrm{SiNa}$ : 379.1700 , found: $379.1700 ;[\alpha]_{\mathrm{D}}^{25}-3.4\left(c 0.50, \mathrm{CHCl}_{3}\right)$. 
HPLC data of 25

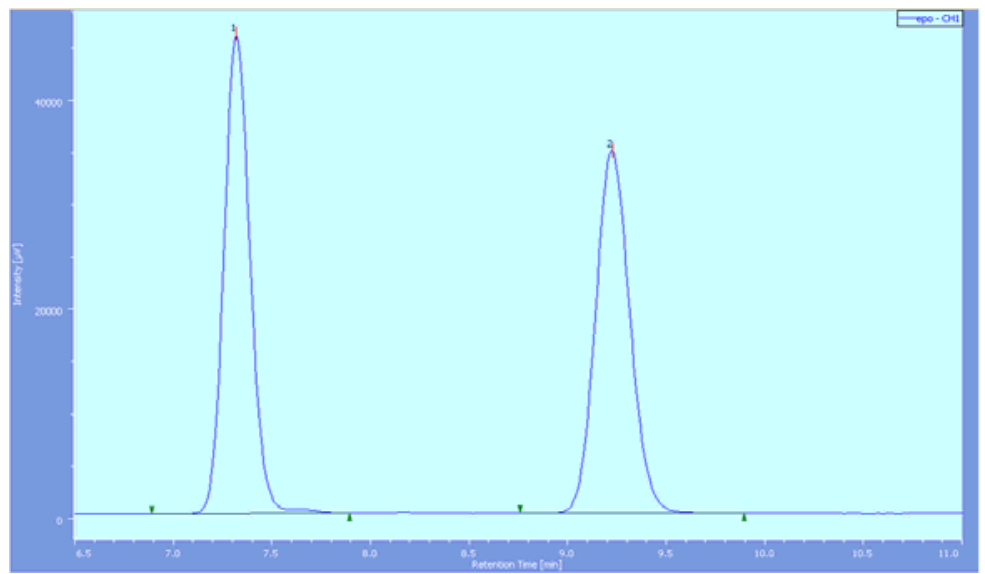

25 (racemate)

\begin{tabular}{|c|c|c|c|c|}
\hline No & TR & Area & Height & Area (\%) \\
\hline 1 & 7.317 & 421343 & 45742 & 50.117 \\
\hline 2 & 9.225 & 419379 & 34627 & 49.883 \\
\hline
\end{tabular}

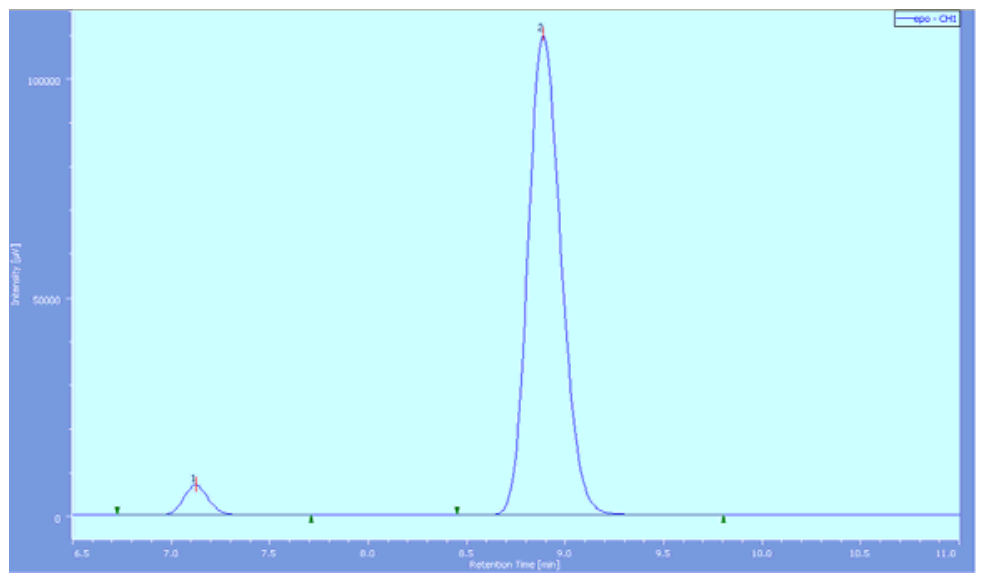

25 (the reaction product)

\begin{tabular}{|c|c|c|c|c|}
\hline No & TR & Area & Height & Area (\%) \\
\hline 1 & 7.125 & 60432 & 6845 & 5.878 \\
\hline 2 & 8.883 & 12816287 & 109610 & 94.122 \\
\hline
\end{tabular}

(2S,3S)-3-tert-butyldiphenylsilyloxymethyl-3-methyloxiran-2-ylmethyl-4-

methylbenzenesulfonate (25b)

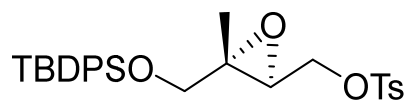

To a stirred solution of $25 \mathrm{a}(43.0 \mathrm{~g}, 121 \mathrm{mmol})$ in $\mathrm{CH}_{2} \mathrm{Cl}_{2}(400 \mathrm{~mL})$ were added $\mathrm{Et}_{3} \mathrm{~N}$ 
(20.2 mL, $145 \mathrm{mmol})$, DMAP (1.50 g, $12.1 \mathrm{mmol})$, and $p$-toluenesulfonyl chloride (27.6 g, 145 $\mathrm{mmol}$ ) at room temperature. The reaction mixture was stirred at room temperature for $3 \mathrm{~h}$, and was quenched with saturated aqueous $\mathrm{NH}_{4} \mathrm{Cl}(100 \mathrm{~mL})$. The aqueous layer was extracted with ethyl acetate $(200 \mathrm{~mL} \times 3)$, and the combined organic layer was washed with brine $(200 \mathrm{~mL})$, dried over $\mathrm{Na}_{2} \mathrm{SO}_{4}$, filtered, and concentrated in vacuo. The residue was purified by flash column chromatography (hexane/ethyl acetate $=30 / 1)$ to afford $25 \mathbf{b}(44.0 \mathrm{~g}, 87.1 \mathrm{mmol}, 72 \%)$ as a white solid: $\mathrm{R}_{\mathrm{f}}=0.30$ (hexane/ethyl acetate $=4 / 1$ ); $\mathrm{mp} 90.0-92.3{ }^{\circ} \mathrm{C} ;{ }^{1} \mathrm{H} \mathrm{NMR}\left(500 \mathrm{MHz}, \mathrm{CDCl}_{3}\right) \delta$ $7.81(\mathrm{~d}, J=8.0 \mathrm{~Hz}, 2 \mathrm{H}), 7.63(\mathrm{~d}, J=8.0 \mathrm{~Hz}, 4 \mathrm{H}), 7.45-7.36(\mathrm{~m}, 6 \mathrm{H}), 7.34(\mathrm{~d}, J=8.5 \mathrm{~Hz}, 2 \mathrm{H})$ $4.17(\mathrm{dd}, J=11.5,5.50 \mathrm{~Hz}, 1 \mathrm{H}), 4.10(\mathrm{dd}, J=11.5,6.5 \mathrm{~Hz}, 1 \mathrm{H}), 3.59$ (s, 2H), 3.13 (dd, $J=6.0$, $5.5 \mathrm{~Hz}, 1 \mathrm{H}), 2.44(\mathrm{~s}, 3 \mathrm{H}), 1.23(\mathrm{~s}, 3 \mathrm{H}), 1.03(\mathrm{~s}, 9 \mathrm{H}) ;{ }^{13} \mathrm{C} \mathrm{NMR}\left(125 \mathrm{MHz}, \mathrm{CDCl}_{3}\right) \delta 145.0,135.6$, 135.5, 133.0, 132.9, 132.8, 129.9, 129.84, 129.82, 128.0, 127.8, 68.4, 66.7, 60.9, 56.1, 26.7, 21.7, 19.2, 14.3; IR (ATR) $v_{\max } 1427,1357,1188,1173,1112,1090,971,854,832,820,774,734,706$, 697, 666, 616, 580, 562, $551 \mathrm{~cm}^{-1}$; HRMS (ESI) $[\mathrm{M}+\mathrm{Na}]^{+}$calcd for $\mathrm{C}_{28} \mathrm{H}_{34} \mathrm{O}_{5} \mathrm{SSiNa}$ : 533.1788, found: $533.1790 ;[\alpha]_{\mathrm{D}}^{25}-18\left(c 0.50, \mathrm{CHCl}_{3}\right)$.

\section{(2S,3S)-3-hydroxymethyl-3-methyloxiran-2-ylmethyl 4-methylbenzenesulfonate (25c)}<smiles>CC(C)(CO)[C@H](O)CO[S+](F)(F)F</smiles>

To a stirred solution of $\mathbf{2 5 b}(44.0 \mathrm{~g}, 87.1 \mathrm{mmol})$ in THF $(280 \mathrm{~mL})$ was added TBAF $(1.0 \mathrm{M}$ in THF, $86.0 \mathrm{~mL}, 86.0 \mathrm{mmol})$ at room temperature. After $30 \mathrm{~min}$, the reaction mixture was quenched with saturated aqueous $\mathrm{NH}_{4} \mathrm{Cl}(100 \mathrm{~mL})$, and the aqueous layer was extracted with ethyl acetate $(200 \mathrm{~mL} \times 3)$. The combined organic layer was washed with brine $(200 \mathrm{~mL})$, dried over $\mathrm{Na}_{2} \mathrm{SO}_{4}$, filtered, and concentrated in vacuo. The residue was purified by flash column chromatography (hexane/ethyl acetate $=2 / 1)$ to afford $25 \mathrm{c}(20.0 \mathrm{~g}, 73.4 \mathrm{mmol}, 84 \%)$ as a colorless oil: $\mathrm{R}_{\mathrm{f}}=0.30$ (hexane/ethyl acetate $\left.=1 / 1\right) ;{ }^{1} \mathrm{H}$ NMR $\left(500 \mathrm{MHz}, \mathrm{CDCl}_{3}\right) \delta 7.82(\mathrm{~d}, J=8.0 \mathrm{~Hz}, 2 \mathrm{H})$, $7.37(\mathrm{~d}, J=8.0 \mathrm{~Hz}, 2 \mathrm{H}), 4.23(\mathrm{dd}, J=11.5,5.50 \mathrm{~Hz}, 1 \mathrm{H}), 4.15(\mathrm{dd}, J=11.5,6.0 \mathrm{~Hz}, 1 \mathrm{H}), 3.66$ (d, $J=12.5 \mathrm{~Hz}, 1 \mathrm{H}), 3.56$ (d, $J=12.5 \mathrm{~Hz}, 1 \mathrm{H}), 3.32(\mathrm{dd}, J=6.5,5.0 \mathrm{~Hz}, 1 \mathrm{H}), 2.46$ (s, 3H), 1.25 (s, 3H); ${ }^{13} \mathrm{C}$ NMR $\left(125 \mathrm{MHz}, \mathrm{CDCl}_{3}\right) \delta 145.3,132.8,130.1,128.1,68.3,64.7,61.1,55.8,21.8$, 14.3; IR (ATR) $v_{\max }$ 3430, 2930, 1598, 1495, 1450, 1354, 1308, 1293, 1211, 1189, 1173, 1095 , 1042, 1020, 958, 850, 814, 763, 685, 663, $552 \mathrm{~cm}^{-1}$; HRMS (ESI) $[\mathrm{M}+\mathrm{Na}]^{+}$calcd for $\mathrm{C}_{12} \mathrm{H}_{16} \mathrm{O}_{5} \mathrm{SNa}: 295.0611$, found: 295.0610; $[\alpha]_{\mathrm{D}}^{25}-31\left(c 0.50, \mathrm{CHCl}_{3}\right)$.

(2S,3R)-3-formyl-3-methyloxiran-2-ylmethyl 4-methylbenzenesulfonate (25) 
<smiles>CC(C)(C=O)CC[OH2+]</smiles>

To a stirred solution of $25 \mathrm{c}(19.0 \mathrm{~g}, 69.8 \mathrm{mmol})$ in $\mathrm{CH}_{2} \mathrm{Cl}_{2}(233 \mathrm{~mL})$ and DMSO (16.3 $\mathrm{mL}, 230 \mathrm{mmol})$ were added $\mathrm{Et}_{3} \mathrm{~N}(58.2 \mathrm{~mL}, 418 \mathrm{mmol})$ and sulfur trioxide pyridine complex $(33.3 \mathrm{~g}, 209 \mathrm{mmol})$ at room temperature. After $1 \mathrm{~h}$, the reaction mixture was quenched with saturated aqueous $\mathrm{NaHCO}_{3}(100 \mathrm{~mL})$ and the aqueous layer was extracted with $\mathrm{CH}_{2} \mathrm{Cl}_{2}(200$ $\mathrm{mL} \times 3)$. The combined organic layer was washed with brine $(200 \mathrm{~mL})$, dried over $\mathrm{Na}_{2} \mathrm{SO}_{4}$, filtered, and concentrated in vacuo. The residue was purified by flash column chromatography (hexane/ethyl acetate $=10 / 1)$ to afford $25(14.0 \mathrm{~g}, 51.8 \mathrm{mmol}, 74 \%)$ as a yellow oil: $\mathrm{R}_{\mathrm{f}}=0.52$ (hexane/ethyl acetate $=1 / 1) ;{ }^{1} \mathrm{H}$ NMR $\left(500 \mathrm{MHz}, \mathrm{CDCl}_{3}\right) \delta 8.80(\mathrm{~s}, 1 \mathrm{H}), 7.82(\mathrm{~d}, J=8.0 \mathrm{~Hz}, 2 \mathrm{H})$, $7.38(\mathrm{~d}, J=8.0 \mathrm{~Hz}, 2 \mathrm{H}), 4.24(\mathrm{dd}, J=11.0,5.5 \mathrm{~Hz}, 1 \mathrm{H}), 4.21(\mathrm{dd}, J=11.0,5.5 \mathrm{~Hz}, 1 \mathrm{H}), 3.44(\mathrm{dd}$, $J=5.5,5.5 \mathrm{~Hz}, 1 \mathrm{H}), 2.47(\mathrm{~s}, 3 \mathrm{H}), 1.37(\mathrm{~s}, 3 \mathrm{H}) ;{ }^{13} \mathrm{C} \mathrm{NMR}\left(125 \mathrm{MHz}, \mathrm{CDCl}_{3}\right) \delta$ 197.6, 145.7, 132.5, 130.2, 128.2, 66.9, 61.7, 56.2, 21.9, 10.1; IR (ATR): $v_{\max } 1731,1598,1361,1190,1174$, 1095, 967, 914, 814, 762, 732, 705, 687, 664, $552 \mathrm{~cm}^{-1}$; HRMS (ESI): $[\mathrm{M}+\mathrm{Na}]^{+}$calcd for $\mathrm{C}_{12} \mathrm{H}_{14} \mathrm{O}_{5} \mathrm{SNa}: 293.0454$, found: 293.0454; $[\alpha]_{\mathrm{D}}^{25}+22\left(c 0.50, \mathrm{CHCl}_{3}\right)$.

\section{Preparation of 23}
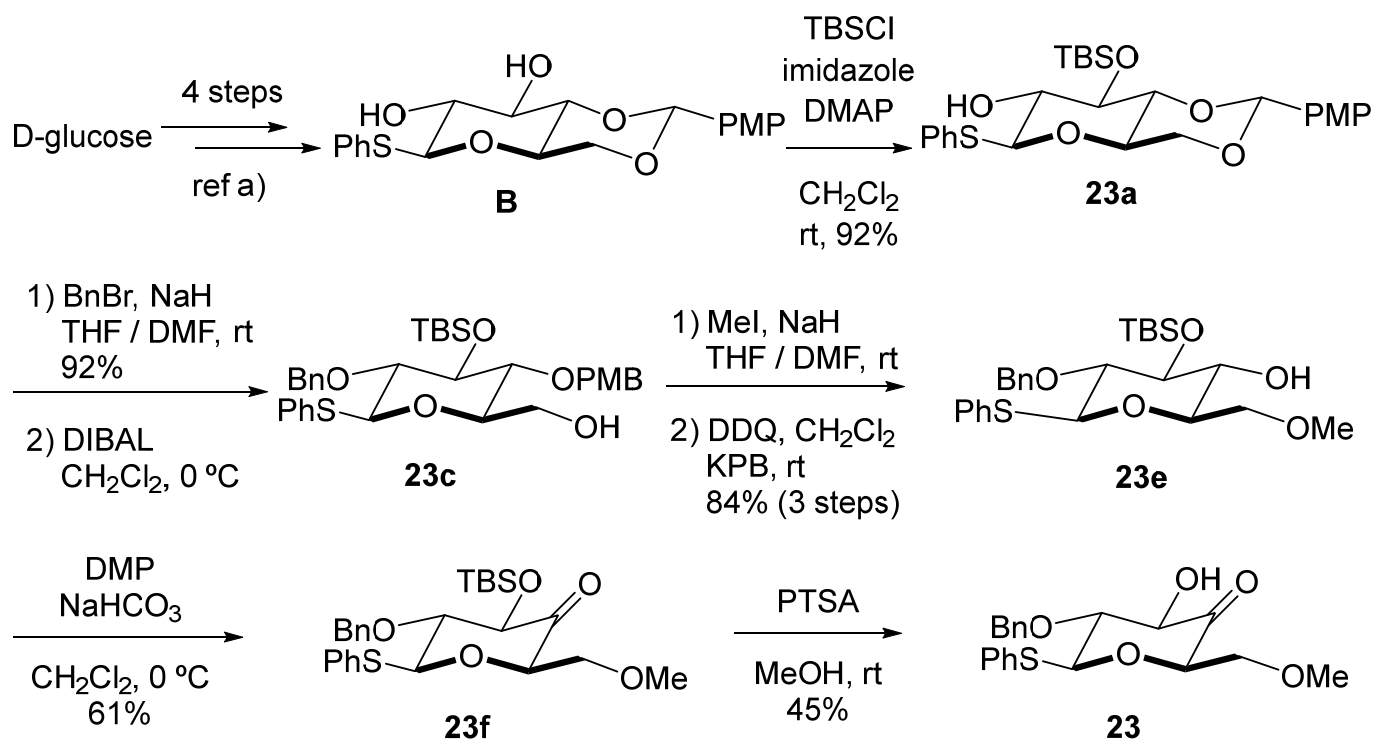

(2R,4aR,6S,7R,8R,8aR)-8-tert-butyldimethylsilyloxy-2-(4-methoxyphenyl)-6phenylthiohexahydropyrano[3,2-d][1,3]dioxin-7-ol (23a) 


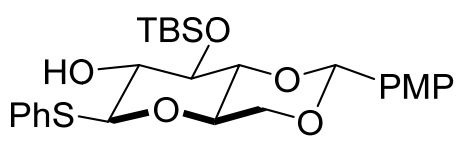

To a stirred solution of diol $\mathbf{B}(5.0 \mathrm{~g}, 12.8 \mathrm{mmol})$ in $\mathrm{CH}_{2} \mathrm{Cl}_{2}(64 \mathrm{~mL})$ were added imidazole (2.62 g, $38.4 \mathrm{mmol})$, DMAP (157 mg, $1.28 \mathrm{mmol})$, and TBSCl (2.90 g, $19.2 \mathrm{mmol})$ at room temperature. After $25 \mathrm{~h}$, the reaction mixture was quenched with saturated aqueous $\mathrm{NH}_{4} \mathrm{Cl}$ $(40 \mathrm{~mL})$, and the aqueous layer was extracted with $\mathrm{CH}_{2} \mathrm{Cl}_{2}(50 \mathrm{~mL} \times 3)$. The combined organic layer was washed with brine $(100 \mathrm{~mL})$, dried over $\mathrm{Na}_{2} \mathrm{SO}_{4}$, filtered, and concentrated in vacuo. The residue was purified by flash column chromatography (hexane/ethyl acetate $=100 / 1$ ) to afford 23a (6.40 g, $12.7 \mathrm{mmol}, 99 \%)$ as a white solid: $\mathrm{R}_{f}=0.33$ (hexane/ethyl acetate $\left.=4 / 1\right)$; $\mathrm{mp}$ 76.7-80.2 ${ }^{\circ} \mathrm{C} ;{ }^{1} \mathrm{H}$ NMR (500 MHz, $\left.\mathrm{CDCl}_{3}\right) \delta$ 7.55-7.52 (m, 2H), 7.41-7.37 (m, 2H), 7.35-7.30 (m, 3H), 6.90-6.86 (m, 2H), $5.46(\mathrm{~s}, 1 \mathrm{H}), 4.65(\mathrm{~d}, J=11.0 \mathrm{~Hz}, 1 \mathrm{H}), 4.35-4.32(\mathrm{~m}, 1 \mathrm{H}), 3.81(\mathrm{~s}$, $3 \mathrm{H}), 3.77(\mathrm{~d}, J=8.0 \mathrm{~Hz}, 1 \mathrm{H}), 3.74(\mathrm{~d}, J=8.0 \mathrm{~Hz}, 1 \mathrm{H}), 3.50-3.40(\mathrm{~m}, 3 \mathrm{H}), 2.47(\mathrm{~d}, J=2.5 \mathrm{~Hz}$, $1 \mathrm{H}), 0.86(\mathrm{~s}, 9 \mathrm{H}), 0.09(\mathrm{~s}, 3 \mathrm{H}), 0.02(\mathrm{~s}, 3 \mathrm{H}) ;{ }^{13} \mathrm{C} \mathrm{NMR}\left(125 \mathrm{MHz}, \mathrm{CDCl}_{3}\right) \delta 160.2,132.9,132.2$, $129.8,129.2,128.3,127.6,113.6,101.8,89.1,81.0,76.1,73.8,71.0,68.7,55.4,26.0,18.5,-4.2$, -4.6; IR (ATR): $v_{\max } 3495,2928,2855,1615,1586,1518,1463,1440,1382,1303,1246,1169$, 1139, 1108, 1067, 1011, 982, 928, 860, 826, 778, 742, 690, 667, 624, 606, 578, $541 \mathrm{~cm}^{-1}$; HRMS (ESI) $[\mathrm{M}+\mathrm{Na}]^{+}$calcd for $\mathrm{C}_{26} \mathrm{H}_{36} \mathrm{O}_{6} \mathrm{SSiNa}: 527.1894$, found: $527.1895 ;[\alpha]_{\mathrm{D}}^{25}-59\left(c 0.50, \mathrm{CHCl}_{3}\right)$.

(2R,4aR,6S,7R,8S,8aR)-7-benzyloxy-2-(4-methoxyphenyl)-6-

phenylthiohexahydropyrano[3,2-d][1,3]dioxin-8-yloxy-tert-butyldimethylsilane (23b)

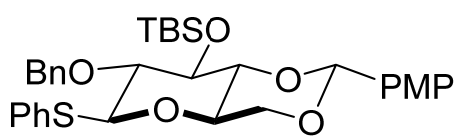

To a stirred solution of 23a $(6.40 \mathrm{~g}, 12.7 \mathrm{mmol})$ in THF $(50 \mathrm{~mL})$ were added $\mathrm{NaH}(60 \%$, $558 \mathrm{mg}, 13.9 \mathrm{mmol}), \mathrm{BnBr}(0.663 \mathrm{~mL}, 13.9 \mathrm{mmol})$, and TBAI (503 mg, $1.27 \mathrm{mmol})$ at $0{ }^{\circ} \mathrm{C}$, and the mixture was allowed to warm to room temperature. After $14 \mathrm{~h}$, the reaction mixture was quenched with saturated aqueous $\mathrm{NH}_{4} \mathrm{Cl}(30 \mathrm{~mL})$ at $0{ }^{\circ} \mathrm{C}$. The mixture was then allowed to warm to room temperature and the aqueous layer was extracted with ethyl acetate $(30 \mathrm{~mL} \times 3)$. The combined organic layer was washed with brine $(100 \mathrm{~mL})$, dried over $\mathrm{Na}_{2} \mathrm{SO}_{4}$, filtered, and concentrated in vacuo. The residue was purified by flash column chromatography (hexane/ethyl acetate $=50 / 1)$ to afford $23 \mathrm{~b}(7.53 \mathrm{~g}, 12.7 \mathrm{mmol}, 100 \%)$ as a white solid: $\mathrm{R}_{\mathrm{f}}=0.44$ (hexane/ethyl

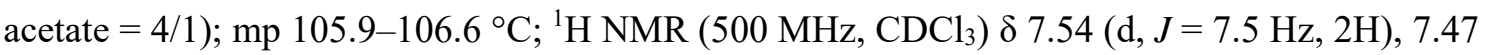
(d, $J=7.5 \mathrm{~Hz}, 2 \mathrm{H}), 7.40(\mathrm{~d}, J=8.5 \mathrm{~Hz}, 2 \mathrm{H}), 7.37-7.29(\mathrm{~m}, 6 \mathrm{H}), 6.89(\mathrm{~d}, J=8.5 \mathrm{~Hz}, 2 \mathrm{H}), 5.46(\mathrm{~s}$, 1H), $4.92(\mathrm{~d}, J=10.0 \mathrm{~Hz}, 1 \mathrm{H}), 4.83(\mathrm{~d}, J=10.0 \mathrm{~Hz}, 1 \mathrm{H}), 4.79(\mathrm{~d}, J=10.5 \mathrm{~Hz}, 1 \mathrm{H}), 4.34(\mathrm{dd}, J$ 
$=10.5,5.0 \mathrm{~Hz}, 1 \mathrm{H}), 3.91(\mathrm{dd}, J=9.5,9.5 \mathrm{~Hz}, 1 \mathrm{H}), 3.81(\mathrm{~s}, 3 \mathrm{H}), 3.77(\mathrm{dd}, J=9.5,9.5 \mathrm{~Hz}, 1 \mathrm{H})$, 3.51-3.40 (m, 3H), 0.87 (s, 9H), 0.045 (s, 3H), -0.009 (s, 3H); $\left.{ }^{13} \mathrm{C} \mathrm{NMR} \mathrm{(125} \mathrm{MHz,} \mathrm{CDCl}_{3}\right) \delta$ $160.2,138.2,133.9,132.0,129.8,129.2,128.4,128.2,127.8,127.8,113.6,102.0,88.8,82.1,81.5$, 76.4, 75.9, 70.4, 68.8, 55.4, 26.0, 18.4, -4.0, -4.3; IR (ATR) $v_{\max } 2928,2855,1615,1586,1518$, $1463,1439,1381,1303,1247,1170,1142,1069,1027,985,927,859,827,778,739,692,668$, 646, 619, 594, $543 \mathrm{~cm}^{-1}$; HRMS (ESI) $[\mathrm{M}+\mathrm{Na}]^{+}$calcd for $\mathrm{C}_{33} \mathrm{H}_{42} \mathrm{O}_{6} \mathrm{SSiNa}: 617.2364$, found: $617.2361 ;[\alpha]_{\mathrm{D}}^{25}-33\left(c 0.50, \mathrm{CHCl}_{3}\right)$.

\section{(2S,3R,4S,5R,6R)-3-benzyloxy-5-(4-methoxybenzyloxy)-6-methoxymethyl-2-} phenylthiotetrahydro-2H-pyran-4-yloxy-tert-butyldimethylsilane (23d)

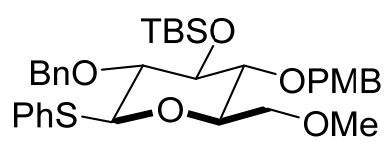

To a stirred solution of $\mathbf{2 3 b}(3.00 \mathrm{~g}, 5.04 \mathrm{mmol})$ in $\mathrm{CH}_{2} \mathrm{Cl}_{2}(16.8 \mathrm{~mL})$ was added DIBAL$\mathrm{H}(1.0 \mathrm{M}$ in hexane, $10.1 \mathrm{~mL}, 10.1 \mathrm{mmol})$ at $0{ }^{\circ} \mathrm{C}$. After $2 \mathrm{~h}$, the reaction mixture was quenched with saturated aqueous $\mathrm{Na}_{2} \mathrm{SO}_{4}(10 \mathrm{~mL})$. After stirring for $3 \mathrm{~h}$, the resulting mixture was filtered through a Celite pad, and the filtrate was concentrated in vacuo. The crude 23c (3.64 g) was used for the next reaction without further purification. To a stirred solution of the crude 23c $(3.64 \mathrm{~g})$ in a mixture of THF (12.6 mL) and DMF (12.6 mL) were added $\mathrm{NaH}(60 \%, 302.4 \mathrm{mg}, 7.56 \mathrm{mmol})$, and then, iodomethane $(0.94 \mathrm{~mL}, 15.1 \mathrm{mmol})$ at room temperature. After $5 \mathrm{~h}$, the reaction mixture was quenched with saturated aqueous $\mathrm{NH}_{4} \mathrm{Cl}(20 \mathrm{~mL})$, and the aqueous layer was extracted with ethyl acetate $(20 \mathrm{~mL} \times 3)$. The combined organic layer was washed with brine $(30 \mathrm{~mL})$, dried over $\mathrm{Na}_{2} \mathrm{SO}_{4}$, filtered, and concentrated in vacuo. The residue was purified by flash column chromatography (hexane/ethyl acetate $=10 / 1)$ to afford 23d $(2.98 \mathrm{~g}, 1.25 \mathrm{mmol}, 4.88 \mathrm{mmol}, 97 \%)$ as a yellow oil: $\mathrm{R}_{\mathrm{f}}=0.45$ (hexane/ethyl acetate $\left.=4 / 1\right) ;{ }^{1} \mathrm{H}$ NMR $\left(500 \mathrm{MHz}, \mathrm{CDCl}_{3}\right) \delta 7.45(\mathrm{~d}, J$ $=7.0 \mathrm{~Hz}, 2 \mathrm{H}), 7.37(\mathrm{~d}, J=7.0 \mathrm{~Hz}, 2 \mathrm{H}), 7.28-7.14(\mathrm{~m}, 8 \mathrm{H}), 6.80(\mathrm{~d}, J=8.5 \mathrm{~Hz}, 2 \mathrm{H}), 4.82(\mathrm{~d}, J$ $=11.0 \mathrm{~Hz}, 1 \mathrm{H}), 4.70(\mathrm{~d}, J=10.5 \mathrm{~Hz}, 1 \mathrm{H}), 4.66(\mathrm{~d}, J=10.5 \mathrm{~Hz}, 1 \mathrm{H}), 4.59(\mathrm{~d}, J=10.0 \mathrm{~Hz}, 1 \mathrm{H})$, $4.46(\mathrm{~d}, J=11.0 \mathrm{~Hz}, 1 \mathrm{H}), 3.72(\mathrm{~s}, 3 \mathrm{H}), 3.65(\mathrm{dd}, J=8.5,8.5 \mathrm{~Hz}, 1 \mathrm{H}), 3.53(\mathrm{dd}, J=11.0,2.0 \mathrm{~Hz}$, $1 \mathrm{H}), 3.49$ (dd, $J=11.0,4.0 \mathrm{~Hz}, 1 \mathrm{H}), 3.41(\mathrm{dd}, J=8.5,8.5 \mathrm{~Hz}, 1 \mathrm{H}), 3.33-3.24$ (m, 2H), 3.28 (s, $3 \mathrm{H}), 0.88(\mathrm{~s}, 9 \mathrm{H}),-0.034(\mathrm{~s}, 3 \mathrm{H}),-0.078(\mathrm{~s}, 3 \mathrm{H}) ;{ }^{13} \mathrm{C} \mathrm{NMR}\left(125 \mathrm{MHz}, \mathrm{CDCl}_{3}\right) \delta 159.2,138.4$, $134.8,131.5,130.6,129.1,129.0,128.3,127.9,127.6,127.3,113.9,88.3,81.7,79.9,78.7,78.5$, 75.3, 74.6, 71.5, 59.4, 55.4, 26.2, 18.1, -3.79, -3.82; IR (ATR): $v_{\max } 2917,2890,2855,1613$, $1514,1472,1462$, 1440, 1301, 1247, 1202, 1145, 1079, 1028, 1004, 898, 835, 777, 739, 692, 671, 635, $597 \mathrm{~cm}^{-1}$; HRMS (ESI): $[\mathrm{M}+\mathrm{Na}]^{+}$calcd for $\mathrm{C}_{34} \mathrm{H}_{46} \mathrm{O}_{6} \mathrm{SSiNa}$ : 633.2677, found: 633.2672; 
$[\alpha]_{\mathrm{D}}^{25}-1.2\left(\right.$ c $\left.0.50, \mathrm{CHCl}_{3}\right)$.

(2R,3R,4S,5R,6S)-5-benzyloxy-4-tert-butyldimethylsilyloxy-3-4-methoxybenzyloxy-6-

phenylthiotetrahydro-2H-pyran-2-ylmethanol (23c)

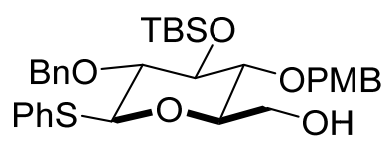

To a stirred solution of $\mathbf{2 3 b}(1.00 \mathrm{~g}, 1.68 \mathrm{mmol})$ in $\mathrm{CH}_{2} \mathrm{Cl}_{2}(16.8 \mathrm{~mL})$ was added DIBAL$\mathrm{H}(1.0 \mathrm{M}$ in hexane, $3.70 \mathrm{~mL}, 3.70 \mathrm{mmol})$ at $0{ }^{\circ} \mathrm{C}$. Then, the mixture was allowed to warm to room temperature. After $2 \mathrm{~h}$, the reaction mixture was quenched with saturated aqueous $\mathrm{Na}_{2} \mathrm{SO}_{4}$ $(10 \mathrm{~mL})$. After stirring for $3 \mathrm{~h}$, the resulting mixture was filtered through a Celite pad, and the filtrate was concentrated in vacuo. The residue was purified by flash column chromatography (hexane/ethyl acetate $=10 / 1)$ to afford $23 \mathrm{c}(897 \mathrm{mg}, 1.50 \mathrm{mmol}, 89 \%)$ as a colorless oil: $\mathrm{R}_{\mathrm{f}}=0.54$ (hexane/ethyl acetate $=2 / 1) ;{ }^{1} \mathrm{H}$ NMR $\left(500 \mathrm{MHz}, \mathrm{CDCl}_{3}\right) \delta 7.49-7.47(\mathrm{~m}, 2 \mathrm{H}), 7.44(\mathrm{~d}, J=7.5$ $\mathrm{Hz}, 2 \mathrm{H}), 7.38-7.24(\mathrm{~m}, 8 \mathrm{H}), 6.87$ (d, $J=8.5 \mathrm{~Hz}, 2 \mathrm{H}), 4.92$ (d, $J=11.0 \mathrm{~Hz}, 1 \mathrm{H}), 4.79$ (d, $J=11.5$ $\mathrm{Hz}, 1 \mathrm{H}), 4.77(\mathrm{~d}, J=11.5 \mathrm{~Hz}, 1 \mathrm{H}), 4.72(\mathrm{~d}, J=10.5 \mathrm{~Hz}, 1 \mathrm{H}), 4.54(\mathrm{~d}, J=11.0 \mathrm{~Hz}, 1 \mathrm{H}), 3.81-$ $3.76(\mathrm{~m}, 1 \mathrm{H}), 3.81(\mathrm{~s}, 3 \mathrm{H}), 3.75(\mathrm{dd}, J=8.5,8.5 \mathrm{~Hz}, 1 \mathrm{H}), 3.60(\mathrm{dd}, J=12.0,5.0 \mathrm{~Hz}, 1 \mathrm{H}), 3.41$ (dd, $J=8.5,8.5 \mathrm{~Hz}, 1 \mathrm{H}), 3.35-3.33$ (m, 1H), 3.32 (dd, $J=10.5,8.5 \mathrm{~Hz}, 1 \mathrm{H}), 0.97$ (s, 9H), 0.07 (s, 3H), $0.01(\mathrm{~s}, 3 \mathrm{H}) ;{ }^{13} \mathrm{C}$ NMR $\left(125 \mathrm{MHz}, \mathrm{CDCl}_{3}\right) \delta 159.4,138.3,134.1,131.6,130.2,129.5$ $129.2,128.4,128.0,127.74,127.67,114.0,88.0,81.9,79.4,78.6,75.5,74.9,62.4,55.4,26.3$, 18.1,-3.76, -3.80; IR (ATR): $v_{\max } 3490,2953,2927,2855,1612,1585,1514,1473,1462,1440$, 1381, 1348, 1302, 1247, 1173, 1151, 1120, 1070, 1028, 935, 886, 835, 777, 738, 692, 671, 635, 607, $573 \mathrm{~cm}^{-1}$; HRMS (ESI) $[\mathrm{M}+\mathrm{Na}]^{+}$calcd for $\mathrm{C}_{33} \mathrm{H}_{44} \mathrm{O}_{6} \mathrm{SSiNa}$ : 619.2520, found: 619.2517; $[\alpha]_{\mathrm{D}}^{25}-11\left(c 0.50, \mathrm{CHCl}_{3}\right)$.

\section{(2S,3R,4S,5R,6R)-3-benzyloxy-5-(4-methoxybenzyloxy)-6-methoxymethyl-2-}

phenylthiotetrahydro-2H-pyran-4-yloxy-tert-butyldimethylsilane (23d)

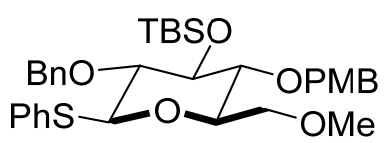

To a stirred solution of 23c ( $878 \mathrm{mg}, 1.47 \mathrm{mmol})$ in a mixture of THF $(14.7 \mathrm{~mL})$ and DMF $(14.7 \mathrm{~mL})$ were added $\mathrm{NaH}(60 \%, 88.4 \mathrm{mg}, 2.21 \mathrm{mmol})$, and then, iodomethane $(0.138 \mathrm{~mL}$, $2.21 \mathrm{mmol}$ ) at room temperature. After $5 \mathrm{~h}$, the reaction mixture was quenched with saturated aqueous $\mathrm{NH}_{4} \mathrm{Cl}(10 \mathrm{~mL})$ and the aqueous layer was extracted with $\mathrm{CH}_{2} \mathrm{Cl}_{2}(15 \mathrm{~mL} \times 3)$. The combined organic layer was washed with brine $(30 \mathrm{~mL})$, dried over $\mathrm{Na}_{2} \mathrm{SO}_{4}$, filtered, and 
concentrated in vacuo. The residue was purified by flash column chromatography (hexane/ethyl acetate $=50 / 1)$ to afford 23d $(764 \mathrm{mg}, 1.25 \mathrm{mmol}, 85 \%)$ as a yellow oil: $\mathrm{R}_{\mathrm{f}}=0.45$ (hexane/ethyl acetate $=4 / 1) ; \mathrm{R}_{\mathrm{f}}=0.45($ hexane/ethyl acetate $=4 / 1) ;{ }^{1} \mathrm{H} \mathrm{NMR}\left(500 \mathrm{MHz}, \mathrm{CDCl}_{3}\right) \delta 7.45(\mathrm{~d}, J=$ $7.0 \mathrm{~Hz}, 2 \mathrm{H}), 7.37$ (d, $J=7.0 \mathrm{~Hz}, 2 \mathrm{H}), 7.28-7.14$ (m, $8 \mathrm{H}), 6.80$ (d, $J=8.5 \mathrm{~Hz}, 2 \mathrm{H}), 4.82$ (d, $J=$ $11.0 \mathrm{~Hz}, 1 \mathrm{H}), 4.70(\mathrm{~d}, J=10.5 \mathrm{~Hz}, 1 \mathrm{H}), 4.66(\mathrm{~d}, J=10.5 \mathrm{~Hz}, 1 \mathrm{H}), 4.59(\mathrm{~d}, J=10.0 \mathrm{~Hz}, 1 \mathrm{H}), 4.46$ $(\mathrm{d}, J=11.0 \mathrm{~Hz}, 1 \mathrm{H}), 3.72(\mathrm{~s}, 3 \mathrm{H}), 3.65(\mathrm{dd}, J=8.5,8.5 \mathrm{~Hz}, 1 \mathrm{H}), 3.53(\mathrm{dd}, J=11.0,2.0 \mathrm{~Hz}, 1 \mathrm{H})$, $3.49(\mathrm{dd}, J=11.0,4.0 \mathrm{~Hz}, 1 \mathrm{H}), 3.41(\mathrm{dd}, J=8.5,8.5 \mathrm{~Hz}, 1 \mathrm{H}), 3.33-3.24(\mathrm{~m}, 2 \mathrm{H}), 3.28(\mathrm{~s}, 3 \mathrm{H})$, $0.88(\mathrm{~s}, 9 \mathrm{H}),-0.034(\mathrm{~s}, 3 \mathrm{H}),-0.078(\mathrm{~s}, 3 \mathrm{H}) ;{ }^{13} \mathrm{C} \mathrm{NMR}\left(125 \mathrm{MHz}, \mathrm{CDCl}_{3}\right) \delta 159.2,138.4,134.8$, 131.5, 130.6, 129.1, 129.0, 128.3, 127.9, 127.6, 127.3, 113.9, 88.3, 81.7, 79.9, 78.7, 78.5, 75.3, 74.6, 71.5, 59.4, 55.4, 26.2, 18.1, -3.79, -3.82; IR (ATR): $v_{\max } 2917,2890,2855,1613,1514$, $1472,1462,1440,1301,1247,1202,1145,1079,1028,1004,898,835,777,739,692,671,635$, $597 \mathrm{~cm}^{-1}$; HRMS (ESI): $[\mathrm{M}+\mathrm{Na}]^{+}$calcd for $\mathrm{C}_{34} \mathrm{H}_{46} \mathrm{O}_{6} \mathrm{SSiNa}$ : 633.2677, found: 633.2672; $[\alpha]_{\mathrm{D}}^{25}$ $-1.2\left(\right.$ c $\left.0.50, \mathrm{CHCl}_{3}\right)$.

\section{(2R,3R,4S,5R,6S)-5-benzyloxy-4-tert-butyldimethylsilyloxy-2-methoxymethyl-6-}

\section{phenylthiotetrahydro-2H-pyran-3-ol (23e)}

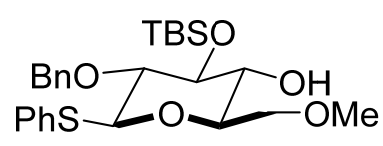

To a stirred solution of $23 \mathbf{d}(6.0 \mathrm{~g}, 9.82 \mathrm{mmol})$ in a mixture of $\mathrm{CH}_{2} \mathrm{Cl}_{2}(90 \mathrm{~mL})$ and aqueous phosphate buffer solution ( $\mathrm{pH} 6.8,9.0 \mathrm{~mL})$ was added DDQ $(3.10 \mathrm{~g}, 13.7 \mathrm{mmol})$ at room temperature. After $2 \mathrm{~h}$, the reaction mixture was quenched with saturated aqueous $\mathrm{NaHCO}_{3}(40$ $\mathrm{mL})$, and the aqueous layer was extracted with $\mathrm{CH}_{2} \mathrm{Cl}_{2}(40 \mathrm{~mL} \times 3)$. The combined organic layer was washed with brine $(100 \mathrm{~mL})$, dried over $\mathrm{Na}_{2} \mathrm{SO}_{4}$, filtered, and concentrated in vacuo. The residue was purified by flash column chromatography (hexane/ethyl acetate $=30 / 1$ ) to afford 23e (4.10 g, $8.36 \mathrm{mmol}, 85 \%)$ as a white solid: $\mathrm{R}_{\mathrm{f}}=0.15$ (hexane/ethyl acetate $\left.=4 / 1\right)$; $\mathrm{mp} 96.1-$ $97.6{ }^{\circ} \mathrm{C} ;{ }^{1} \mathrm{H}$ NMR $\left(500 \mathrm{MHz}, \mathrm{CDCl}_{3}\right) \delta 7.53-7.49$ (m, 2H), 7.44 (d, J=7.5 Hz, 2H), 7.37-7.24 (m, 6H), 4.91 (d, $J=10.5 \mathrm{~Hz}, 1 \mathrm{H}), 4.72(\mathrm{~d}, J=10.5 \mathrm{~Hz}, 1 \mathrm{H}), 4.68$ (d, $J=9.5 \mathrm{~Hz}, 1 \mathrm{H}), 3.71$ (dd, $J=9.5,5.0 \mathrm{~Hz}, 1 \mathrm{H}), 3.67(\mathrm{dd}, J=9.5,5.0 \mathrm{~Hz}, 1 \mathrm{H}), 3.63(\mathrm{dd}, J=8.5,8.5 \mathrm{~Hz}, 1 \mathrm{H}), 3.56(\mathrm{dd}, J=$ 8.5, 8.5 Hz, 1H), 3.44-3.39 (m, 1H), 3.41 (s, 3H), 3.32 (dd, $J=9.5,8.5 \mathrm{~Hz}, 1 \mathrm{H}), 0.93$ (s, 9H), $0.11(\mathrm{~s}, 3 \mathrm{H}), 0.059$ (s, 3H); ${ }^{13} \mathrm{C} \mathrm{NMR}\left(125 \mathrm{MHz}, \mathrm{CD}_{2} \mathrm{Cl}_{2}\right) \delta 138.9,135.0,131.5,129.3,128.5$, $128.1,127.8,127.6,88.3,81.2,79.6,77.9,75.3,73.3,72.9,59.7,26.2,18.5,-4.0,-4.1$; IR (ATR) $v_{\max } 3547,2923,2852,1584,1497,1481,1471,1455,1440,1403,1378,1358,1303,1278,1245$, 1212, 1198, 1141, 1119, 1066, 1049, 1025, 979, 969, 941, 919, 886, 836, 779, 762, 738, 707, 697 , 
688, 669, 630, 570, 552, $530 \mathrm{~cm}^{-1}$; HRMS (ESI) $[\mathrm{M}+\mathrm{Na}]^{+}$calcd for $\mathrm{C}_{26} \mathrm{H}_{38} \mathrm{O}_{5} \mathrm{SSiNa}$ : 513.2101, found: $513.2102 ;[\alpha]_{\mathrm{D}}^{25}-33\left(c 0.50, \mathrm{CHCl}_{3}\right)$.

$(2 R, 4 R, 5 R, 6 S)-5$-benzyloxy-4-tert-butyldimethylsilyloxy-2-methoxymethyl-6phenylthiodihydro-2H-pyran-3(4H)-one (23f)

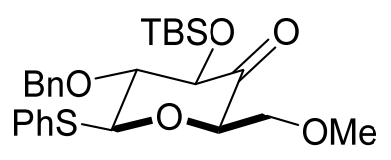

To a stirred solution of DMSO $(2.33 \mathrm{~mL}, 32.9 \mathrm{mmol})$ in $\mathrm{CH}_{2} \mathrm{Cl}_{2}(40 \mathrm{~mL})$ was added oxalyl chloride $(2.25 \mathrm{~mL}, 26.3 \mathrm{mmol})$ at $-78^{\circ} \mathrm{C}$. After $10 \mathrm{~min}$, to the mixture was added $23 \mathbf{e}(4.76$ $\mathrm{g}, 8.76 \mathrm{mmol})$ in $\mathrm{CH}_{2} \mathrm{Cl}_{2}(47 \mathrm{~mL})$ and the mixture was stirred at the same temperature for $30 \mathrm{~min}$. To the reaction mixture was added triethylamine $(9.16 \mathrm{~mL}, 65.7 \mathrm{mmol})$, and the resultant mixture was stirred at room temperature for $2 \mathrm{~h}$. The reaction mixture was quenched with saturated aqueous $\mathrm{NH}_{4} \mathrm{Cl}(90 \mathrm{~mL})$, and the aqueous layer was extracted with $\mathrm{CH}_{2} \mathrm{Cl}_{2}(50 \mathrm{~mL} \times 3)$. The combined organic layer was washed with brine $(100 \mathrm{~mL})$, dried over $\mathrm{Na}_{2} \mathrm{SO}_{4}$, filtered, and concentrated in vacuo. The residue was purified by flash column chromatography (hexane/ethyl acetate $=40 / 1)$ to afford $23 \mathrm{f}(3.47 \mathrm{~g}, 7.10 \mathrm{mmol}, 81 \%)$ as a white solid: $\mathrm{R}_{\mathrm{f}}=0.73$ (hexane/ethyl acetate $=2 / 1) ; \mathrm{mp} 79.7-82.0{ }^{\circ} \mathrm{C} ;{ }^{1} \mathrm{H}$ NMR $\left(500 \mathrm{MHz}, \mathrm{CDCl}_{3}\right) \delta 7.58-7.55(\mathrm{~m}, 2 \mathrm{H}), 7.44(\mathrm{~d}, J=$ $6.9 \mathrm{~Hz}, 2 \mathrm{H}), 7.38-7.27(\mathrm{~m}, 6 \mathrm{H}), 5.04(\mathrm{~d}, J=9.0 \mathrm{~Hz}, 1 \mathrm{H}), 4.83$ (d, $J=10.5 \mathrm{~Hz}, 1 \mathrm{H}), 4.80(\mathrm{~d}, J=$ $10.5 \mathrm{~Hz}, 1 \mathrm{H}), 4.35$ (d, $J=9.0 \mathrm{~Hz}, 1 \mathrm{H}), 4.09$ (dd, $J=6.0,3.5 \mathrm{~Hz}, 1 \mathrm{H}), 3.90$ (dd, $J=11.5,3.5 \mathrm{~Hz}$, $1 \mathrm{H}), 3.70(\mathrm{dd}, J=9.0,9.0 \mathrm{~Hz}, 1 \mathrm{H}), 3.64(\mathrm{dd}, J=11.5,6.0 \mathrm{~Hz}, 1 \mathrm{H}), 3.38$ (s, 3H), 0.95 (s, 9H), $0.13(\mathrm{~s}, 3 \mathrm{H}), 0.04(\mathrm{~s}, 3 \mathrm{H}) ;{ }^{13} \mathrm{C} \mathrm{NMR}\left(125 \mathrm{MHz}, \mathrm{CDCl}_{3}\right) \delta 200.9,137.7,133.8,132.1,129.2,128.5$, $128.4,128.0,127.9,88.2,83.9,80.8,80.2,75.6,70.5,59.6,25.9,18.6,-4.5,-5.1$; IR (ATR) $v_{\max }$ 2929, 2856, 1738, 1481, 1472, 1441, 1249, 1213, 1158, 1102, 1077, 1044, 1017, 997, 909, 836, $813,777,753,742,701,692,620,582,565 \mathrm{~cm}^{-1}$; HRMS (ESI): $[\mathrm{M}+\mathrm{Na}]^{+}$calcd for $\mathrm{C}_{26} \mathrm{H}_{36} \mathrm{O}_{5} \mathrm{SSiNa}$ : 511.1945, found: 511.1946; $[\alpha]_{\mathrm{D}}^{25}-17$ (c 0.50, $\left.\mathrm{CHCl}_{3}\right)$.

\section{(2R,4R,5R,6S)-5-benzyloxy-4-hydroxy-2-methoxymethyl-6-phenylthiodihydro-2H-pyran-}

\section{3(4H)-one (23)}

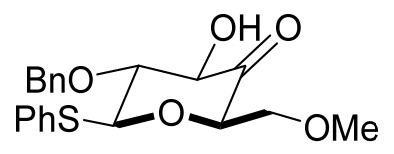

To a stirred solution of $23 \mathrm{f}(1.22 \mathrm{~g}, 2.50 \mathrm{mmol})$ in $\mathrm{MeOH}(25.0 \mathrm{~mL})$ was added $p$ - 
toluensulfonic acid monohydrate $(475 \mathrm{mg}, 2.50 \mathrm{mmol}$ ) at room temperature. After $8 \mathrm{~h}$, the reaction mixture was quenched with saturated aqueous $\mathrm{NaHCO}_{3}(10 \mathrm{~mL})$ and the aqueous layer was extracted with $\mathrm{Et}_{2} \mathrm{O}(30 \mathrm{~mL} \times 3)$. The combined organic layer was washed with brine $(40 \mathrm{~mL})$, dried over $\mathrm{Na}_{2} \mathrm{SO}_{4}$, filtered, and concentrated in vacuo. The residue was purified by silica gel column chromatography (hexane/ethyl acetate $=15 / 1)$ to afford $23(438 \mathrm{mg}, 1.17 \mathrm{mmol}, 47 \%)$ as a yellow oil: $\mathrm{R}_{\mathrm{f}}=0.23$ (hexane/ethyl acetate $\left.=2 / 1\right) ;{ }^{1} \mathrm{H} \mathrm{NMR}\left(500 \mathrm{MHz}, \mathrm{CDCl}_{3}\right) \delta 7.57-7.54(\mathrm{~m}$, 2H), $7.46(\mathrm{~d}, J=7.0 \mathrm{~Hz}, 2 \mathrm{H}), 7.37(\mathrm{t}, J=7.0 \mathrm{~Hz}, 2 \mathrm{H}), 7.33-7.29(\mathrm{~m}, 4 \mathrm{H}), 5.06(\mathrm{~d}, J=9.5 \mathrm{~Hz}$, $1 \mathrm{H}), 4.92(\mathrm{~d}, J=10.5 \mathrm{~Hz}, 1 \mathrm{H}), 4.81(\mathrm{~d}, J=10.5 \mathrm{~Hz}, 1 \mathrm{H}), 4.40(\mathrm{~d}, J=9.5 \mathrm{~Hz}, 1 \mathrm{H}), 4.17(\mathrm{dd}, J=$ $6.5,4.0 \mathrm{~Hz}, 1 \mathrm{H}), 3.91(\mathrm{dd}, J=11.0,4.0 \mathrm{~Hz}, 1 \mathrm{H}), 3.66(\mathrm{dd}, J=11.0,6.5 \mathrm{~Hz}, 1 \mathrm{H}), 3.63(\mathrm{dd}, J=$ 9.5, $9.5 \mathrm{~Hz}, 1 \mathrm{H}), 3.38(\mathrm{~s}, 3 \mathrm{H}) ;{ }^{13} \mathrm{C} \mathrm{NMR}\left(125 \mathrm{MHz}, \mathrm{CDCl}_{3}\right) \delta 201.8,137.6,133.0,132.3,129.0$, 128.4, 128.3, 128.0, 127.9, 87.3, 83.8, 79.6, 79.4, 74.7, 69.8, 59.5; IR (ATR): $v_{\max } 3430,2892$, $1732,1583,1497,1479,1454,1439,1242,1203,1075,1043,1026,988,902,836,737,692,553$ $\mathrm{cm}^{-1}$; HRMS (ESI) $[\mathrm{M}+\mathrm{Na}]^{+}$calcd for $\mathrm{C}_{20} \mathrm{H}_{22} \mathrm{O}_{5} \mathrm{SNa}$ : 397.1080, found: 397.1081; $[\alpha]_{\mathrm{D}}^{25}-14(c$ $\left.0.1, \mathrm{CHCl}_{3}\right)$

\section{(2S,3S,5R,7S,8R,8aR)-8-benzyloxy-5-methoxymethyl-3-methyl-3-((S)-oxiran-2-yl)-7-}

phenylthiotetrahydro-5H,7H-2,4a-epoxypyrano[3,4-b][1,4]dioxine (24)

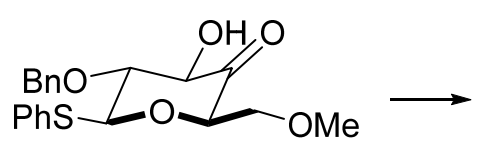

23

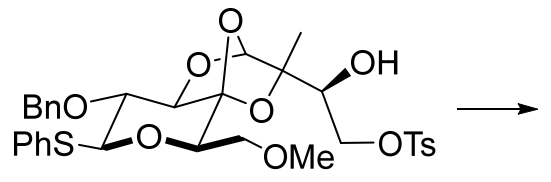

28

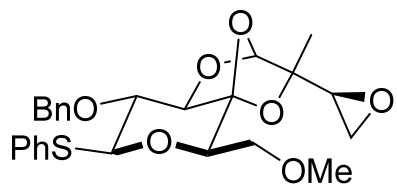

24

To a stirred mixture of $23(708 \mathrm{mg}, 1.89 \mathrm{mmol})$ and $25(511 \mathrm{mg}, 1.89 \mathrm{mmol})$ in $\mathrm{CH}_{3} \mathrm{CN}$ $(0.95 \mathrm{~mL})$ was added $(+)-C S A(439.0 \mathrm{mg}, 1.89 \mathrm{mmol})$ at room temperature. The reaction mixture was stirred at room temperature for $24 \mathrm{~h}$, and then quenched with saturated aqueous $\mathrm{NaHCO}_{3}(20$ $\mathrm{mL})$. The aqueous layer was extracted with $\mathrm{CH}_{2} \mathrm{Cl}_{2}(20 \mathrm{~mL} \times 3)$, and the combined organic layer was washed with brine $(40 \mathrm{~mL})$, dried over $\mathrm{Na}_{2} \mathrm{SO}_{4}$, filtered, and concentrated in vacuo. The residue was roughly purified by short silica gel column chromatography to afford crude $\mathbf{2 8}$ as a colorless oil, which was used for the next reaction without further purification.

To a stirred solution of the crude $\mathbf{2 8}$ in THF (18.9 mL) was added NaH (60\%, $75.6 \mathrm{mg}$, $1.89 \mathrm{mmol}$ ) at $0{ }^{\circ} \mathrm{C}$. After $30 \mathrm{~min}$, the reaction mixture was quenched with saturated aqueous $\mathrm{NH}_{4} \mathrm{Cl}(10 \mathrm{~mL})$, and the aqueous layer was extracted with ethyl acetate $(10 \mathrm{~mL} \times 3)$. The combined organic layer was washed with brine $(30 \mathrm{~mL})$, dried over $\mathrm{Na}_{2} \mathrm{SO}_{4}$, filtered, and concentrated in vacuo. The residue was purified by flash column chromatography (hexane/ethyl acetate $=30 / 1$ ) 
to afford 24 ( $206 \mathrm{mg}, 0.436 \mathrm{mmol}, 23 \%$ ( 2 steps)) as a yellow oil: $\mathrm{R}_{\mathrm{f}}=0.51$ (hexane/ethyl acetate $=2 / 1) ;{ }^{1} \mathrm{H} \mathrm{NMR}\left(500 \mathrm{MHz}, \mathrm{CDCl}_{3}\right) \delta 7.56-7.54(\mathrm{~m}, 2 \mathrm{H}), 7.42(\mathrm{~d}, J=7.0 \mathrm{~Hz}, 2 \mathrm{H}), 7.35(\mathrm{t}, J=$ $7.5 \mathrm{~Hz}, 2 \mathrm{H}), 7.31-7.26(\mathrm{~m}, 4 \mathrm{H}), 5.60(\mathrm{~s}, 1 \mathrm{H}), 4.76(\mathrm{~d}, J=11.0 \mathrm{~Hz}, 1 \mathrm{H}), 4.69(\mathrm{~d}, J=10.0 \mathrm{~Hz}, 1 \mathrm{H})$, $4.67(\mathrm{~d}, J=11.0 \mathrm{~Hz}, 1 \mathrm{H}), 4.05(\mathrm{dd}, J=8.0$ and $2.0 \mathrm{~Hz}, 1 \mathrm{H}), 3.99(\mathrm{~d}, J=6.5 \mathrm{~Hz}, 1 \mathrm{H}), 3.82(\mathrm{dd}, J$ $=11.0,2.0 \mathrm{~Hz}, 1 \mathrm{H}), 3.72(\mathrm{dd}, J=11.0,8.0 \mathrm{~Hz}, 1 \mathrm{H}), 3.37(\mathrm{~s}, 3 \mathrm{H}), 3.34(\mathrm{dd}, J=10.0,6.5 \mathrm{~Hz}, 1 \mathrm{H})$, $3.16(\mathrm{dd}, J=3.5,3.0 \mathrm{~Hz}, 1 \mathrm{H}), 2.83-2.80(\mathrm{~m}, 2 \mathrm{H}), 1.04(\mathrm{~s}, 3 \mathrm{H}) ;{ }^{13} \mathrm{C} \mathrm{NMR}\left(125 \mathrm{MHz}, \mathrm{CDCl}_{3}\right) \delta$ 137.7, 133.1, 132.2, 128.9, 128.3, 128.0, 127.8, 127.7, 105.9, 104.1, 86.1, 85.5, 84.5, 79.1, 74.7, 73.6, 69.9, 59.4, 52.5, 44.1, 18.1; IR (ATR) $v_{\max } 2930,1735,1583,1497,1480,1454,1439,1401$, $1371,1339,1267,1243,1202,1109,1026,987,952,923,895,870,854,825,735,693,646,555$ $\mathrm{cm}^{-1}$; HRMS (ESI) $[\mathrm{M}+\mathrm{Na}]^{+}$calcd for $\mathrm{C}_{25} \mathrm{H}_{28} \mathrm{O}_{7} \mathrm{SNa}$ : 495.1448, found: 495.1450; $[\alpha]_{\mathrm{D}}^{25}+17(c$ $\left.0.63, \mathrm{CHCl}_{3}\right)$.

\section{Gram-scale preparation of 24}

To a stirred mixture of $23(5.48 \mathrm{~g}, 15.9 \mathrm{mmol})$ and 25 (4.30 g, $15.9 \mathrm{mmol})$ in $\mathrm{CH}_{3} \mathrm{CN}$ $(7.0 \mathrm{~mL})$ was added $(+)-\mathrm{CSA}(439.0 \mathrm{mg}, 1.89 \mathrm{mmol})$ at room temperature. The reaction mixture was stirred at room temperature for $24 \mathrm{~h}$, and was quenched with saturated aqueous $\mathrm{NaHCO}_{3}(100$ $\mathrm{mL})$. The aqueous layer was extracted with $\mathrm{CH}_{2} \mathrm{Cl}_{2}(200 \mathrm{~mL} \times 3)$, and the combined organic layer was washed with brine $(200 \mathrm{~mL})$, dried over $\mathrm{Na}_{2} \mathrm{SO}_{4}$, filtered, and concentrated in vacuo. The residue was roughly purified by short silica gel column chromatography to afford crude $\mathbf{2 8}$ as a colorless oil, which was used for the next reaction without further purification.

To a stirred solution of the crude 28 in THF $(160 \mathrm{~mL})$ was added $\mathrm{NaH}(60 \%, 636 \mathrm{mg}$, $15.9 \mathrm{mmol}$ ) at $0{ }^{\circ} \mathrm{C}$. After $30 \mathrm{~min}$, the reaction mixture was quenched with saturated aqueous $\mathrm{NH}_{4} \mathrm{Cl}(100 \mathrm{~mL})$ and the aqueous layer was extracted with ethyl acetate $(100 \mathrm{~mL} \times 3)$. The organic layer was washed with brine $(200 \mathrm{~mL})$, dried over $\mathrm{Na}_{2} \mathrm{SO}_{4}$, filtered, and concentrated in vacuo. The residue was purified by flash column chromatography (hexane/ethyl acetate $=30 / 1$ ) to afford 24 (1.60 g, $3.39 \mathrm{mmol}, 21 \%$ (2 steps)) as a yellow oil. 


\section{Enantioselective Total Synthesis of Cotylenin A}

$(1 R, 3 a S, 4 R, 5 R, 6 R, 9 a R, E)-6$-hydroxy-7-isopropyl-1-methoxymethyl-4,9a-dimethyl-1trimethylsilyloxy-1,2,3,3a,4,5,6,8,9,9a-decahydrodicyclopenta[a,d][8]annulen-5-yl acetate (29)

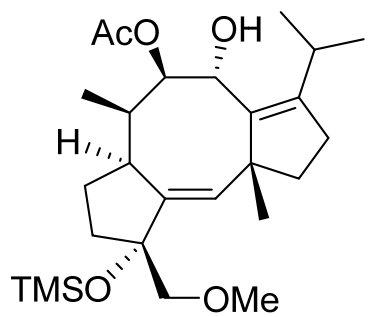

To a stirred solution of $21(91.1 \mathrm{mg}, 0.215 \mathrm{mmol})$ in pyridine $(4.3 \mathrm{~mL})$ was added $\mathrm{Ac}_{2} \mathrm{O}(48.7 \mu \mathrm{L}, 0.431 \mathrm{mmol})$ at room temperature. The reaction mixture was stirred at room temperature for $4 \mathrm{~h}$, and then $\mathrm{Ac}_{2} \mathrm{O}(73.1 \mu \mathrm{L}, 0.647 \mathrm{mmol})$ was added to the mixture. After $4 \mathrm{~h}$, the reaction mixture was quenched with saturated aqueous $\mathrm{NaHCO}_{3}(30 \mathrm{~mL})$, and the aqueous layer was extracted with ethyl acetate $(50 \mathrm{~mL} \times 3)$. The combined organic layer was washed with brine $(50 \mathrm{~mL})$, dried over $\mathrm{Na}_{2} \mathrm{SO}_{4}$, filtered, and concentrated in vacuo. The residue was purified by flash column chromatography (hexane/ethyl acetate $=10 / 1)$ to afford $29(58.8 \mathrm{mg}, 0.127 \mathrm{mmol}$, $59 \%$ [77\%, brsm]) as a colorless oil, diacetate 29a (16.7 mg, $0.033 \mathrm{mmol}, 15 \%)$, and 21 (21.4 mg, $0.0506 \mathrm{mmol}, 24 \%)$.

29: $\mathrm{R}_{\mathrm{f}}=0.40$ (hexane/ethyl acetate $\left.=2 / 1\right) ;{ }^{1} \mathrm{H}$ NMR $\left(500 \mathrm{MHz}, \mathrm{CDCl}_{3}\right) \delta 5.67($ br $1 \mathrm{H}), 5.53-5.50$ (m, 1H), $4.30(\mathrm{~d}, J=10.3 \mathrm{~Hz}, 1 \mathrm{H}), 3.73-3.62(\mathrm{~m}, 1 \mathrm{H}), 3.27(\mathrm{~d}, J=9.7 \mathrm{~Hz}, 1 \mathrm{H}), 3.15(\mathrm{~s}, 3 \mathrm{H})$, 3.13-3.05 (m, 1H), 2.99 (d, $J=9.7 \mathrm{~Hz}, 1 \mathrm{H}), 2.24-2.18$ (m, 2H), 2.08-1.91 (m, 3H), 1.76-1.69 (m, 2H), $1.73(\mathrm{~s}, 3 \mathrm{H}), 1.62-1.52(\mathrm{~m}, 1 \mathrm{H}), 1.47-1.36(\mathrm{~m} \mathrm{1H}), 1.24(\mathrm{~s}, 3 \mathrm{H}), 1.11$ (d, J=6.9 Hz, $3 \mathrm{H}), 1.10-0.95(\mathrm{~m}, 1 \mathrm{H}), 1.02(\mathrm{~d}, J=6.9 \mathrm{~Hz}, 3 \mathrm{H}), 0.86(\mathrm{~d}, J=6.9 \mathrm{~Hz}, 3 \mathrm{H}), 0.25(\mathrm{~s}, 9 \mathrm{H}) ;{ }^{13} \mathrm{C} \mathrm{NMR}$ $\left(125 \mathrm{MHz}, \mathrm{CDCl}_{3}\right) \delta 170.0,151.0,140.4,136.2,134.5,85.5,79.9,77.6,65.8,58.9,52.0,43.3$, 40.3, 40.1, 34.3, 31.3, 28.4, 27.4, 26.3, 21.5, 20.9, 20.4, 9.2, 2.5; IR (ATR) $v_{\max } 3496,2955,2875$, $2365,2155,2022,1742,1715,1449,1367,1247,1053,840 \mathrm{~cm}^{-1}$; HRMS (ESI) $[\mathrm{M}+\mathrm{Na}]^{+}$calcd for $\mathrm{C}_{26} \mathrm{H}_{44} \mathrm{O}_{5} \mathrm{SiNa}$ : 487.2853, found: 487.2850; $[\alpha]_{\mathrm{D}}^{25}-77$ (c 1.05, MeOH).

29a: $\mathrm{R}_{\mathrm{f}}=0.70$ (hexane/ethyl acetate $\left.=2 / 1\right) ;{ }^{1} \mathrm{H}$ NMR $\left(500 \mathrm{MHz}, \mathrm{CDCl}_{3}\right) \delta 5.47(\mathrm{~d}, J=10.9 \mathrm{~Hz}$, $1 \mathrm{H}), 5.42(\mathrm{~d}, J=2.9 \mathrm{~Hz}, 1 \mathrm{H}), 5.30(\mathrm{dd}, J=10.9,4.6 \mathrm{~Hz}, 1 \mathrm{H}), 3.36(\mathrm{~s}, 3 \mathrm{H}), 3.29-3.22(\mathrm{~m}, 2 \mathrm{H})$, 2.92-2.88 (m, 2H), 2.14-2.00 (m, 4H), 2.03 (s, 3H), 1.98 (s, 3H), 1.95-1.78 (m, 3H), 1.69-1.62 (m, 1H), 1.43-1.36 (m, 1H), 1.33-1.25 (m, 1H), $1.28(\mathrm{~s}, 3 \mathrm{H}), 1.20-1.11(\mathrm{~m}, 1 \mathrm{H}), 0.99(\mathrm{~d}, J=6.9$ $\mathrm{Hz}, 3 \mathrm{H}), 0.98(\mathrm{~d}, J=6.9 \mathrm{~Hz}, 3 \mathrm{H}), 0.86(\mathrm{~d}, J=6.9 \mathrm{~Hz}, 3 \mathrm{H}), 0.076(\mathrm{~s}, 9 \mathrm{H}) ;{ }^{13} \mathrm{C} \mathrm{NMR}(125 \mathrm{MHz}$, 
$\left.\mathrm{CDCl}_{3}\right) \delta 170.2,169.4,152.5,139.5,134.3,132.4,85.1,76.9,67.9,59.3,51.7,42.9,39.8,39.7$, $33.5,31.2,28.0,27.1,25.7,21.2,21.1,21.0,20.3,8.9,2.2$; IR (ATR) $v_{\max } 2956,1744,1450,1367$, 1205, 1052, 1027, $839 \mathrm{~cm}^{-1}$; HRMS (ESI) $[\mathrm{M}+\mathrm{Na}]^{+}$calcd for $\mathrm{C}_{28} \mathrm{H}_{46} \mathrm{O}_{6} \mathrm{NaSi}$ : 529.2952, found: $529.2956 ;[\alpha]_{\mathrm{D}}^{25}-202\left(c 1.15, \mathrm{CH}_{2} \mathrm{Cl}_{2}\right)$.

$(1 R, 3 a S, 4 R, 5 R, 6 R, 9 a R, E)-6-((2 S, 3 S, 5 R, 7 S, 8 R, 8 \mathrm{a} R)-8$-benzyloxy-5-methoxymethyl-3methyl-3-((S)-oxiran-2-yl)tetrahydro-5H,7H-2,4a-epoxypyrano[3,4-b][1,4]dioxin-7-yloxy)7-isopropyl-1-methoxymethyl-4,9a-dimethyl-1-trimethylsilyloxy-1,2,3,3a,4,5,6,8,9,9adecahydrodicyclopenta[a,d][8]annulen-5-yl acetate (30)
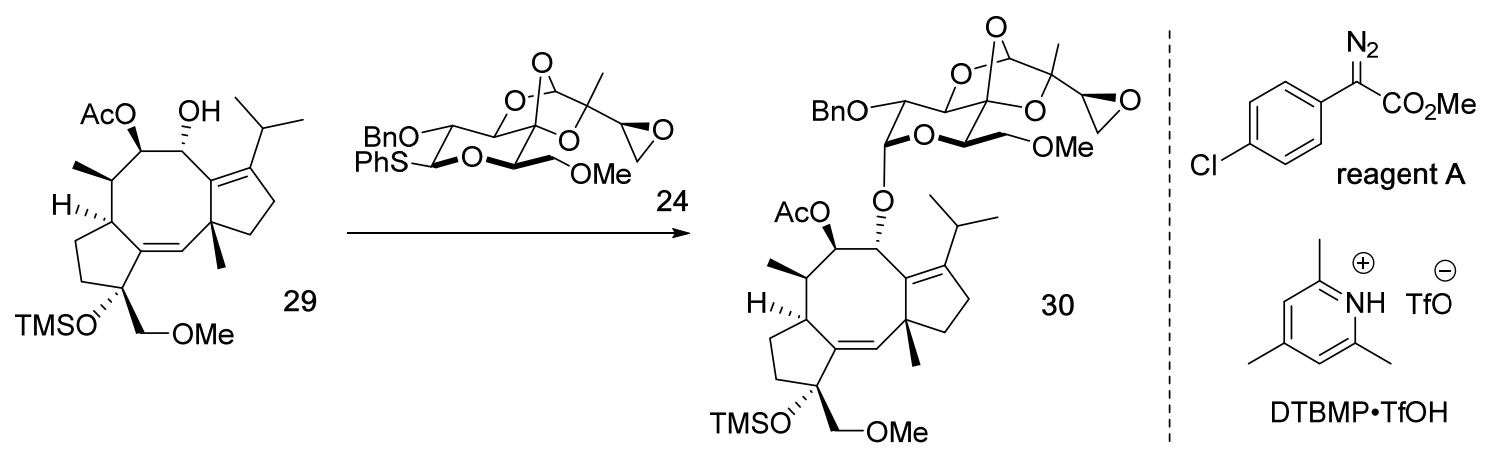

To a stirred mixture of $29(77.5 \mathrm{mg}, 0.167 \mathrm{mmol})$ and $24(94.6 \mathrm{mg}, 0.200 \mathrm{mmol})$ in $\mathrm{CH}_{2} \mathrm{Cl}_{2}(3.3 \mathrm{~mL})$ were added MS3 $\AA$ (100 mg), reagent $\mathrm{A}(105.5 \mathrm{mg}, 0.500 \mathrm{mmol})$ and DTBMP•TfOH $(5.9 \mathrm{mg}, 16.7 \mu \mathrm{mol})$ at room temperature. Then, to the mixture was added $\mathrm{Rh}_{2}$ (oct) $)_{4}\left(2 \mathrm{mg} / \mathrm{mL}\right.$ in $\left.\mathrm{CH}_{2} \mathrm{Cl}_{2}, 0.33 \mathrm{~mL}, 0.834 \mu \mathrm{mol}\right)$ at $0{ }^{\circ} \mathrm{C}$. The reaction mixture was stirred at $0{ }^{\circ} \mathrm{C}$ for $2 \mathrm{~h}$, and then quenched with saturated aqueous $\mathrm{NaHCO}_{3}(10 \mathrm{~mL})$. The mixture was filtrated through a short Celite pad and the aqueous layer was extracted with ethyl acetate (10 $\mathrm{mL} \times 3)$. The combined organic layer was washed with brine $(20 \mathrm{~mL})$, dried over $\mathrm{Na}_{2} \mathrm{SO}_{4}$, filtered, and concentrated in vacuo. The residue was purified by flash column chromatography (hexane $/ \mathrm{CH}_{2} \mathrm{Cl}_{2}=3: 1$ to hexane/ethyl acetate $\left.=10 / 1\right)$ to afford crude $30(51.7 \mathrm{mg}$ including inseparable 29 and unknown impurities) as a colorless oil. The mixture was used for the next reaction without further purification: $\mathrm{R}_{\mathrm{f}}=0.65$ (hexane/ethyl acetate $=2 / 1$ ).

$(1 R, 3 \mathrm{a} S, 4 R, 5 R, 6 R, 9 \mathrm{a} R, E)-6-(((2 S, 3 S, 5 R, 7 S, 8 R, 8 \mathrm{a} R)-8$-benzyloxy-5-methoxymethyl-3methyl-3-((S)-oxiran-2-yl)tetrahydro-5H,7H-2,4a-epoxypyrano[3,4-b][1,4]dioxin-7-yl)oxy)7-isopropyl-1-methoxymethyl-4,9a-dimethyl-1-trimethylsilyloxy-1,2,3,3a,4,5,6,8,9,9adecahydrodicyclopenta[a,d][8]annulen-5-ol (31) 


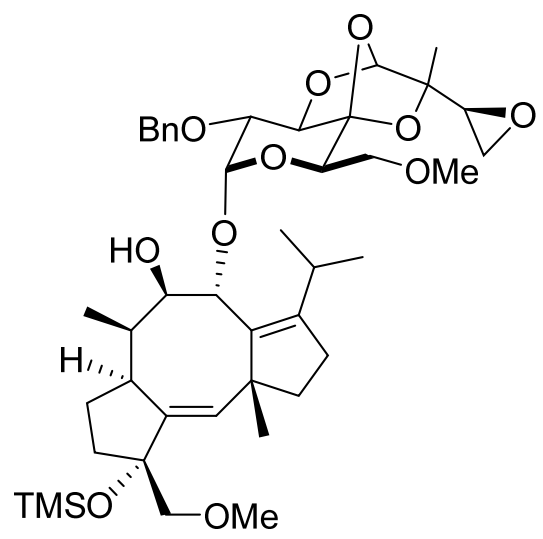

To a stirred solution of the crude 30 (ca. $51.7 \mathrm{mg}$ ) in $\mathrm{Et}_{2} \mathrm{O}(1.3 \mathrm{~mL}$ ) was added $\mathrm{MeLi}$ $\left(1.09 \mathrm{M}\right.$ in $\left.\mathrm{Et}_{2} \mathrm{O}, 1.3 \mathrm{~mL}, 0.625 \mathrm{mmol}\right)$ at $-78^{\circ} \mathrm{C}$. The reaction mixture was gradually warmed up to $-30{ }^{\circ} \mathrm{C}$ and stirred for $2 \mathrm{~h}$. The mixture was quenched with saturated aqueous $\mathrm{NaHCO}_{3}(15$ $\mathrm{mL})$. The aqueous layer was extracted with ethyl acetate $(10 \mathrm{~mL} \times 3)$. The combined organic layer was washed with brine $(20 \mathrm{~mL})$, dried over $\mathrm{Na}_{2} \mathrm{SO}_{4}$, filtered, and concentrated in vacuo. The crude $31(54.3 \mathrm{mg})$ was used for the next reaction without further purification.

$(1 R, 3 \mathrm{a} S, 4 R, 5 R, 6 R, 9 \mathrm{a} R, E)-6-(((2 S, 3 S, 5 R, 7 S, 8 R, 8 \mathrm{a} R)-8$-benzyloxy-5-methoxymethyl-3methyl-3-((S)-oxiran-2-yl)tetrahydro-5H,7H-2,4a-epoxypyrano[3,4-b][1,4]dioxin-7-yl)oxy)7-isopropyl-1-methoxymethyl-4,9a-dimethyl-1,2,3,3a,4,5,6,8,9,9adecahydrodicyclopenta[a,d][8]annulene-1,5-diol (32)

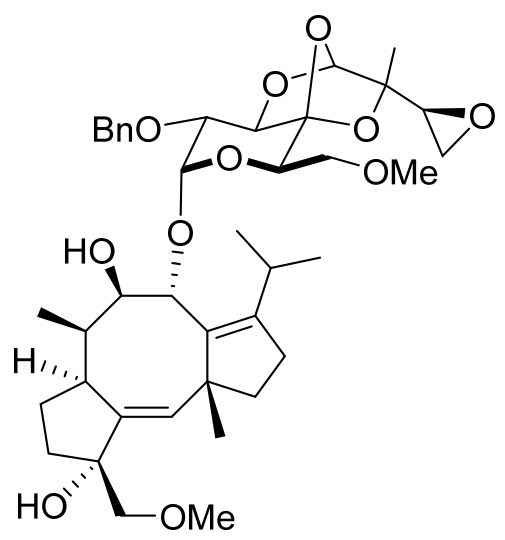

To a stirred solution of the crude 31 (ca. $54.3 \mathrm{mg}$ ) in THF $(1.3 \mathrm{~mL}$ ) was added TBAF (1.0 M in THF, $0.31 \mathrm{~mL}, 0.313 \mathrm{mmol}$ ) at room temperature. The reaction mixture was stirred at room temperature for $4 \mathrm{~h}$, and quenched with saturated aqueous $\mathrm{NH}_{4} \mathrm{Cl}$ solution $(15 \mathrm{~mL})$. The aqueous layer was extracted with ethyl acetate $(10 \mathrm{~mL} \times 3)$, and the combined organic layer was washed with brine $(10 \mathrm{~mL})$, dried over $\mathrm{Na}_{2} \mathrm{SO}_{4}$, filtered, and concentrated under reduced pressure. The residue was purified by flash silica gel $60 \mathrm{NH}_{2}$ chromatography (dichloromethane / ethyl 
acetate $=100 / 1)$ to afford cotylenol $(13.7 \mathrm{mg}, 0.0391 \mathrm{mmol})$ and $32(21.4 \mathrm{mg}$ that includes inseparable unknown impurities) as a colorless oil. The mixture was used next reaction without further purification: $\mathrm{R}_{\mathrm{f}}=0.40$ (hexane/ethyl acetate $\left.=1 / 1\right) ;{ }^{1} \mathrm{H} \mathrm{NMR}\left(500 \mathrm{MHz}, \mathrm{CDCl}_{3}\right) \delta 7.38-$ $7.23(\mathrm{~m}, 24 \mathrm{H}), 5.69$ (d, $J=1.7 \mathrm{~Hz}, 1 \mathrm{H}), 5.59(\mathrm{~s}, 1 \mathrm{H}), 5.57$ (s, 2.3H), $5.51(\mathrm{~d}, J=2.3 \mathrm{~Hz}, 2.3 \mathrm{H})$, $5.40(\mathrm{~d}, J=3.4 \mathrm{~Hz}, 1 \mathrm{H}), 5.01(\mathrm{~d}, J=3.4 \mathrm{~Hz}, 2.3 \mathrm{H}), 4.83(\mathrm{~d}, J=12.0 \mathrm{~Hz}, 2.3 \mathrm{H}), 4.68(\mathrm{~d}, J=12.0$ $\mathrm{Hz}, 2.3 \mathrm{H}), 4.65$ (br 2H), 4.54 (dd, $J=7.4,2.9 \mathrm{~Hz}, 1 \mathrm{H}), 4.34$ (dd, J=7.2, 2.6 Hz, 2.3H), 4.27 (dd, $J=10.3,4.6 \mathrm{~Hz}, 1 \mathrm{H}), 4.20-4.11(\mathrm{~m}, 4.4 \mathrm{H}), 3.95(\mathrm{dd}, J=10.3,4.0 \mathrm{~Hz}, 2.3 \mathrm{H}), 3.88(\mathrm{~d}, J=9.7 \mathrm{~Hz}$, 2.3H), 3.79-3.63 (m, 8.6H), 3.43-3.31 (m, 30H), 3.22-3.12 (m, 6.8H), 3.07 (d, J=9.2 Hz, 2.3H), 2.92-2.76 (m, 10H), 2.70-2.65 (m, 1H), 2.51 (br, 2H), 2.39 (br, 1H), 2.24-2.18 (br, 2H), 2.131.48 (m, 30H), 1.42-1.20 (m, 28H), 1.16 (d, $J=13.2 \mathrm{~Hz}, 3 \mathrm{H}), 1.07$ (d, $J=6.3 \mathrm{~Hz}, 6.9 \mathrm{H}), 1.05-$ $1.02(\mathrm{~m}, 9 \mathrm{H}), 1.00(\mathrm{~d}, J=6.9 \mathrm{~Hz}, 3 \mathrm{H}), 0.96(\mathrm{~d}, J=6.9 \mathrm{~Hz}, 6.9 \mathrm{H}), 0.90-0.85(\mathrm{~m}, 9 \mathrm{H}), 0.84-0.77$ $(\mathrm{m}, 9 \mathrm{H}) ;{ }^{13} \mathrm{C} \mathrm{NMR}\left(125 \mathrm{MHz}, \mathrm{CDCl}_{3}\right) \delta 149.8,139.3,137.6,137.3,134.7,134.6,128.5,128.2$, $128.0,127.5,126.9,121.2,106.1,103.9,103.8,100.6,91.4,85.2,82.1,81.9,81.3,80.9,78.9$, $78.5,78.3,78.2,78.0,74.6,72.8,71.7,70.0,69.8,66.2,65.7,59.3,59.3,59.2,53.0,52.6,52.4$, 51.8, 44.1, 42.4, 40.9, 40.4, 39.8, 39.6, 37.2, 36.4, 35.2, 32.0, 31.6, 31.5, 28.1, 27.5, 26.8, 26.6, 25.5, 25.4, 23.6, 22.6, 21.4, 21.3, 21.2, 20.7, 18.3, 17.9, 14.1, 8.7, 8.3, -0.0; IR (ATR) $v_{\max } 3499$, 2930, 1454, 1372, 1299, 1245, 1098, 1076, 1053, 1018, 956, 922, 894, 750, 698, $667 \mathrm{~cm}^{-1}$; HRMS (ESI) $[\mathrm{M}+\mathrm{Na}]^{+}$calcd for $\mathrm{C}_{40} \mathrm{H}_{56} \mathrm{O}_{11} \mathrm{Na}: 735.3708$, found: 735.3715

\section{Cotylenin A}

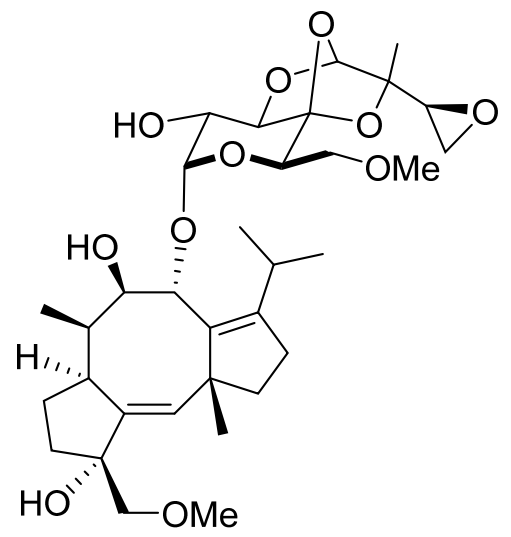

To a stirred solution of 32 (ca. $21.4 \mathrm{mg}, 31.3 \mu \mathrm{mol})$ in THF $(3.1 \mathrm{~mL})$ was added Pd black (33 mg, $0.313 \mathrm{mmol}$ ) at room temperature. The mixture was stirred under an atmosphere of $\mathrm{H}_{2}$ (balloon) at $45^{\circ} \mathrm{C}$ for $15 \mathrm{~h}$. The mixture was filtered through a Celite pad and the residue was washed with ethyl acetate. The combined filtrate was concentrated under reduced pressure. The residue was purified by silica gel $60 \mathrm{NH}_{2}$ chromatography (hexane/ethyl acetate $=3 / 1$ to $1 / 1$ ) to 
afford cotylenin A (5.7 mg, $9.15 \mu \mathrm{mol}, 6 \%$ (7\% brsm), 4 steps from 29$)$ as a white solid: $R_{f}=0.4$ (hexane/ethyl acetate $=1 / 2) ; \mathrm{mp} 95-97{ }^{\circ} \mathrm{C} ;{ }^{1} \mathrm{H}$ NMR $\left(600 \mathrm{MHz}, \mathrm{CDCl}_{3}\right) \delta 5.64(\mathrm{~s}, 1 \mathrm{H}), 5.53(\mathrm{~d}$, $J=2.6 \mathrm{~Hz}, 1 \mathrm{H}), 5.09$ (d, $J=3.3 \mathrm{~Hz}, 1 \mathrm{H}), 4.36(\mathrm{dd}, J=6.7,3.6 \mathrm{~Hz}, 1 \mathrm{H}), 4.06(\mathrm{~d}, J=8.2 \mathrm{~Hz}, 1 \mathrm{H})$, $4.01(\mathrm{dd}, J=10.1,4.2 \mathrm{~Hz}, 1 \mathrm{H}), 3.93$ (d, $J=10.1 \mathrm{~Hz}, 1 \mathrm{H}), 3.78$ (br, $1 \mathrm{H}), 3.75-3.68$ (m, 2H), 3.63$3.61(\mathrm{~m}, 1 \mathrm{H}), 3.41(\mathrm{~s}, 3 \mathrm{H}), 3.37(\mathrm{~s}, 3 \mathrm{H}), 3.35(\mathrm{~d}, J=9.5 \mathrm{~Hz}, 1 \mathrm{H}), 3.20-3.13(\mathrm{~m}, 1 \mathrm{H}), 3.13(\mathrm{t}, J=$ $3.3 \mathrm{~Hz}, 1 \mathrm{H}), 3.08$ (d, $J=9.5 \mathrm{~Hz}, 1 \mathrm{H}), 2.93$ (dd, $J=8.6,2.6 \mathrm{~Hz}, 1 \mathrm{H}), 2.85$ (br, 1H), 2.81 (d, $J=$ $3.3 \mathrm{~Hz}, 2 \mathrm{H}), 2.52$ (br, 1H), 2.12-2.07 (m, 2H), 2.04-1.89 (m, 3H), 1.87-1.79 (m, 1H), 1.76-1.69 (m, 1H), 1.45-1.36 (m, 1H), 1.30 (s, 3H), 1.32-1.22 (m, 1H), 1.09 (d, J = 6.7 Hz, 3H), 1.06 (s, $3 \mathrm{H}), 0.97(\mathrm{~d}, J=6.9 \mathrm{~Hz}, 3 \mathrm{H}), 0.80(\mathrm{~d}, J=7.0 \mathrm{~Hz}, 3 \mathrm{H}) ;{ }^{13} \mathrm{C} \mathrm{NMR}\left(150 \mathrm{MHz}, \mathrm{CDCl}_{3}\right) \delta 149.9$, 139.3, 134.74, 134.72, 106.0, 104.0, 101.3, 85.3, 81.9, 81.3, 78.4, 78.0, 77.3, 73.0, 70.0, 66.3, $59.30,59.27,52.7,51.9,44.1,42.4,41.5,40.4,35.2,31.6,28.2,26.9,25.5,21.5,20.6,18.5,8.3$; IR (ATR) $v_{\max }$ 3456, 2927, 1727, 1455, 1374, 1298, 1261, 1118, 1078, 1013, 958, $754 \mathrm{~cm}^{-}$ ${ }^{1}$;HRMS (ESI) $[\mathrm{M}+\mathrm{Na}]^{+}$calcd for $\mathrm{C}_{33} \mathrm{H}_{50} \mathrm{O}_{11} \mathrm{Na}$ : 645.3243, found: 645.3245; $[\alpha]_{\mathrm{D}}^{25}+31$ (c 0.28, $\left.\mathrm{CHCl}_{3}\right)$. 


\section{Comparison of NMR Data between Synthetic and Natural Cotylenin A}

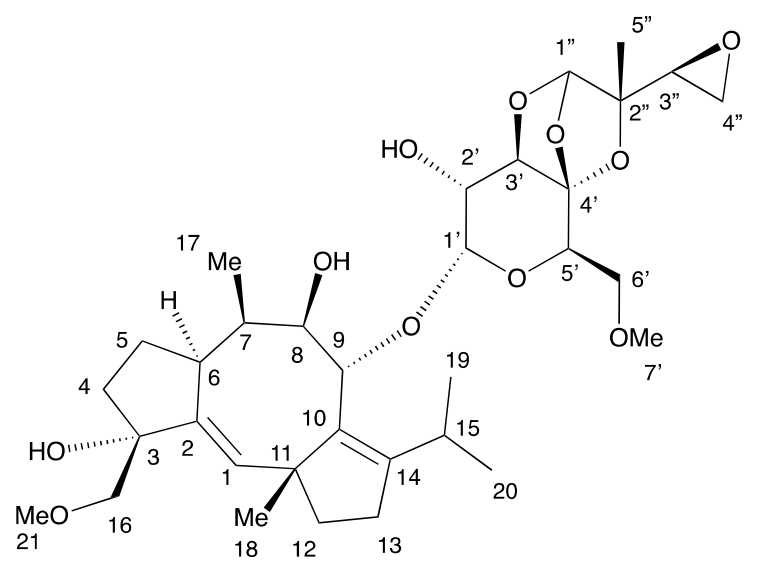

\begin{tabular}{|c|c|c|c|c|}
\hline & \multicolumn{4}{|c|}{ natural $[\mathrm{CDCl} 3600 \mathrm{MHz}]$} \\
\hline & $\mathrm{H}$ & & $\mathrm{J}(\mathrm{Hz})$ & $13 \mathrm{C}$ \\
\hline 1 & 5.53 & d & 2.6 & 134.76 \\
\hline 2 & & & & 139.30 \\
\hline 3 & & & & 81.97 \\
\hline \multirow{2}{*}{4} & 1.40 & $b r, d d$ & $12,12,7$ & \multirow{2}{*}{35.26} \\
\hline & 2.01 & $\mathrm{~m}$ & & \\
\hline \multirow{2}{*}{5} & 1.26 & $\mathrm{~m}$ & & \multirow{2}{*}{31.60} \\
\hline & 1.98 & $\mathrm{~m}$ & & \\
\hline 6 & 2.93 & dd & $8.6,2.6$ & 40.36 \\
\hline 7 & 1.93 & qd & $7.1,4.0$ & 41.46 \\
\hline 8 & 4.01 & dd & $10.1,4.0$ & 78.42 \\
\hline 9 & 3.93 & d & 10.1 & 78.00 \\
\hline 10 & & & & 134.76 \\
\hline 11 & & & & 51.92 \\
\hline \multirow{2}{*}{12} & 1.725 & ddd & $11.8,9.9,8.5$ & \multirow{2}{*}{42.43} \\
\hline & 1.825 & ddd & $11.8,6.2,2.6$ & \\
\hline 13 & $2.12-2.07$ & $\mathrm{~m}, 2 \mathrm{H}$ & & 26.88 \\
\hline 14 & & & & 149.94 \\
\hline 15 & 3.163 & br, sep, & 6.8 & 28.22 \\
\hline \multirow{2}{*}{16} & 3.35 & $d$ & 9.5 & \multirow{2}{*}{77.34} \\
\hline & 3.08 & dd & $9.5,1.2$ & \\
\hline 17 & 0.80 & $\mathrm{~d}, 3 \mathrm{H}$ & 7.1 & 8.36 \\
\hline 18 & 1.30 & $\mathrm{~s}, 3 \mathrm{H}$ & & 25.57 \\
\hline 19 & 1.09 & $\mathrm{~d}, 3 \mathrm{H}$ & 6.6 & 21.53 \\
\hline 20 & 0.97 & $\mathrm{~d}, 3 \mathrm{H}$ & 7.0 & 20.59 \\
\hline 21 & 3.37 & \multicolumn{2}{|l|}{$\mathrm{s}, 3 \mathrm{H}$} & 59.31 \\
\hline $1^{\prime}$ & 5.09 & $d$ & 3.5 & 101.39 \\
\hline $2^{\prime}$ & 3.62 & ddd & $8.2,5.0,3.5$ & 72.82 \\
\hline 3 & 4.06 & $d$ & 8.2 & 81.33 \\
\hline $4^{\prime}$ & & & & 106.05 \\
\hline $5^{\prime}$ & 4.36 & dd & $6.8,3.7$ & 66.31 \\
\hline $6^{\prime}$ & $3.72-3.67$ & \multicolumn{2}{|l|}{$\mathrm{m}, 2 \mathrm{H}$} & 70.04 \\
\hline 7 & 3.41 & $\mathrm{~s}, 3 \mathrm{H}$ & & 59.27 \\
\hline $1^{\prime \prime}$ & 5.57 & \multirow{2}{*}{\multicolumn{2}{|c|}{0.5}} & 103.97 \\
\hline $2^{\prime \prime}$ & & & & 85.24 \\
\hline $3^{\prime \prime}$ & 3.15 & \multicolumn{2}{|r|}{$3.6,3.0$} & 52.72 \\
\hline $4^{\prime \prime}$ & $2.83-2.79$ & $\mathrm{~m}, 2 \mathrm{H}$ & & 44.15 \\
\hline $5^{\prime \prime}$ & 1.06 & \multicolumn{2}{|l|}{$\mathrm{s}, 3 \mathrm{H}$} & 18.45 \\
\hline
\end{tabular}

\begin{tabular}{|c|c|c|c|c|}
\hline & \multicolumn{4}{|c|}{ synthetic [CDCl3 600MHz] } \\
\hline & $\mathrm{H}$ & & $\mathrm{J}(\mathrm{Hz})$ & $13 \mathrm{C}$ \\
\hline 1 & 5.53 & $\mathrm{~d}$ & 2.6 & 134.74 \\
\hline 2 & & & & 139.29 \\
\hline 3 & & & & 81.92 \\
\hline \multirow{2}{*}{4} & $1.45-1.36$ & $m$ & & \multirow{2}{*}{35.24} \\
\hline & $2.04-1.89$ & $\mathrm{~m}, 1 \mathrm{H}$ & & \\
\hline \multirow{2}{*}{5} & $1.32-1.22$ & $\mathrm{~m}$ & & \multirow{2}{*}{31.58} \\
\hline & $2.04-1.89$ & $\mathrm{~m}, 1 \mathrm{H}$ & & \\
\hline 6 & 2.93 & dd & 8.62 .6 & 40.35 \\
\hline 7 & $2.04-1.89$ & $\mathrm{~m}, 1 \mathrm{H}$ & & 41.52 \\
\hline 8 & 4.01 & $\mathrm{dd}$ & 10.14 .2 & 78.39 \\
\hline 9 & 3.93 & d & 10.1 & 77.98 \\
\hline 10 & & & & 134.72 \\
\hline 11 & & & & 51.91 \\
\hline \multirow{2}{*}{12} & $1.76-1.69$ & $\mathrm{~m}$ & & \multirow{2}{*}{42.42} \\
\hline & $1.87-1.79$ & $\mathrm{~m}$ & & \\
\hline 13 & $2.12-2.07$ & $\mathrm{~m}$ & & 26.86 \\
\hline 14 & & & & 149.94 \\
\hline 15 & $3.20-3.13$ & $\mathrm{~m}$ & & 28.22 \\
\hline \multirow{2}{*}{16} & 3.35 & $d$ & 9.5 & \multirow{2}{*}{77.30} \\
\hline & 3.08 & $d$ & 9.5 & \\
\hline 17 & 0.80 & $\mathrm{~d}, 3 \mathrm{H}$ & 7.0 & 8.32 \\
\hline 18 & 1.30 & $\mathrm{~s}, 3 \mathrm{H}$ & & 25.53 \\
\hline 19 & 1.09 & $\mathrm{~d}, 3 \mathrm{H}$ & 6.7 & 21.51 \\
\hline 20 & 0.97 & $\mathrm{~d}, 3 \mathrm{H}$ & 6.9 & 20.58 \\
\hline 21 & 3.37 & $\mathrm{~s}, 3 \mathrm{H}$ & & 59.30 \\
\hline $1^{\prime}$ & 5.09 & $d$ & 3.3 & 101.25 \\
\hline $2^{\prime}$ & $3.63-3.61$ & $\mathrm{~m}, 1 \mathrm{H}$ & & 72.97 \\
\hline $3^{\prime}$ & 4.06 & d & 8.2 & 81.27 \\
\hline $4^{\prime}$ & & & & 106.02 \\
\hline $5^{\prime}$ & 4.36 & $\mathrm{dd}$ & 6.73 .6 & 66.32 \\
\hline $6^{\prime}$ & $3.75-3.68$ & $\mathrm{~m}, 2 \mathrm{H}$ & & 70.02 \\
\hline $7^{\prime}$ & 3.41 & $\mathrm{~s}, 3 \mathrm{H}$ & & 59.27 \\
\hline $1^{\prime \prime}$ & 5.56 & $\mathrm{~s}$ & & 103.99 \\
\hline $2^{\prime \prime}$ & & & & 85.26 \\
\hline $3 "$ & 3.13 & $t$ & 3.3 & 52.68 \\
\hline $4^{\prime \prime}$ & 2.82 & $\mathrm{~d}, 2 \mathrm{H}$ & 3.3 & 44.11 \\
\hline $5^{\prime \prime}$ & 1.06 & $\mathrm{~s}, 3 \mathrm{H}$ & & 18.46 \\
\hline
\end{tabular}




\section{References}

1. Katsuki, T.; Sharpless, B. The First Practical Method for Asymmetric Epoxidation. J. Am. Chem. Soc. 1980, 102, 5974-5976.

2. Yamano, Y.; Nishiyama, Y.; Aoki, A.; Maoka, T.; Wada, A. Total synthesis of lycopene-5,6diol and $\gamma$-carotene-5', $6^{\prime}$-diol stereoisomers and their HPLC separation. Tetrahedron 2017, 73, 2043-2052.

3. Maruoka, K.; Ooi, T.; Nagahara, S.; Yamamoto, H. Organoaluminum-catalyzed rearrangement of epoxides a facile route to the synthesis of optically active $\beta$-siloxy aldehydes. Tetrahedron 1991, 47, 6983-6998.

4. Shi, L.; Lei, X.; Zhang, J.; Lin, G. Synthesis of Racemic $\Delta^{3}-2$-Hydroxybakuchiol and Its Analogues. Helvetica Chim. Acta 2010, 93, 555-564.

5. Ikeda, S.; Shibuya, M.; Iwabuchi, Y. Asymmetric total synthesis of martinelline and martinellic acid. Chem. Commun. 2007, 5, 504-506. 


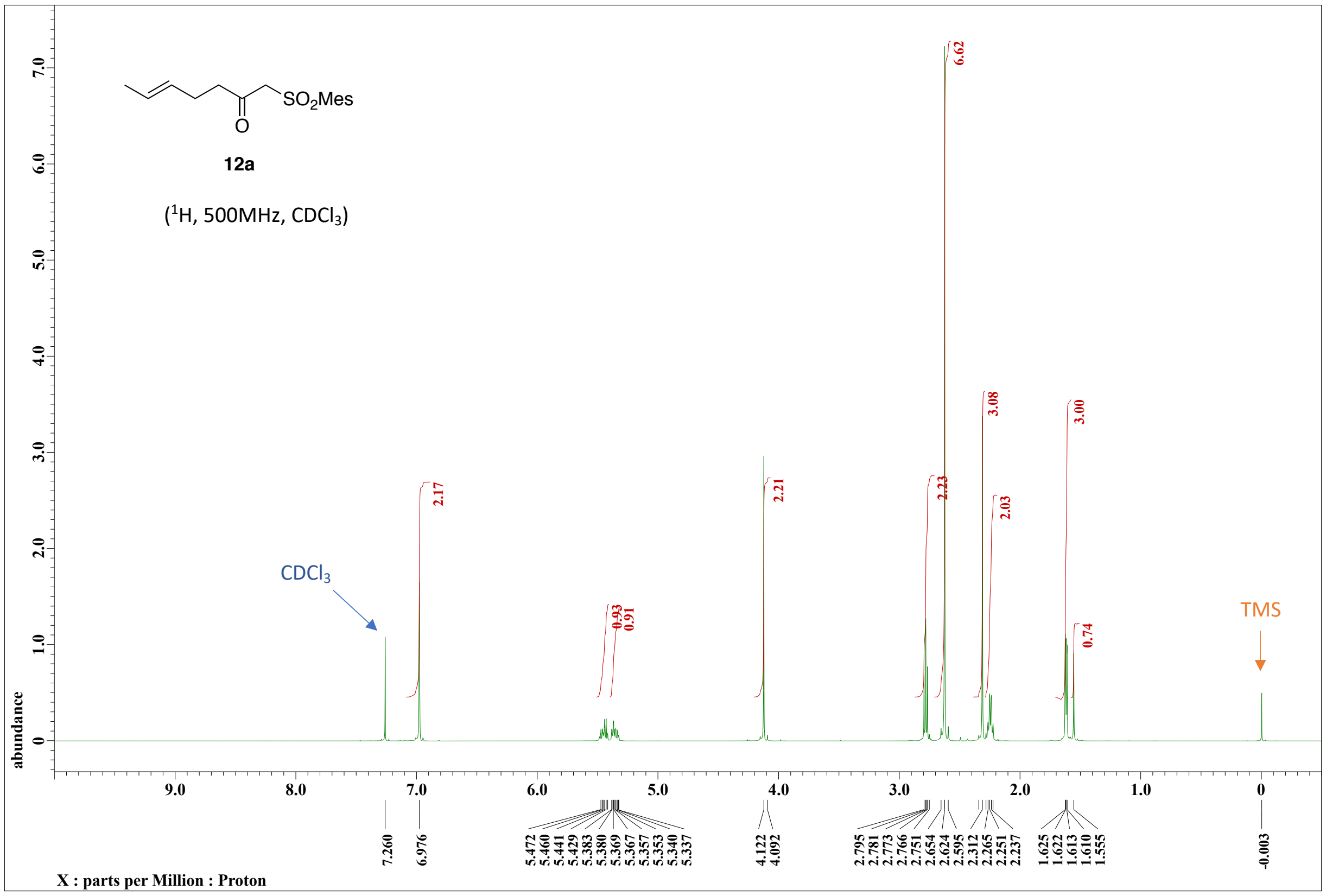




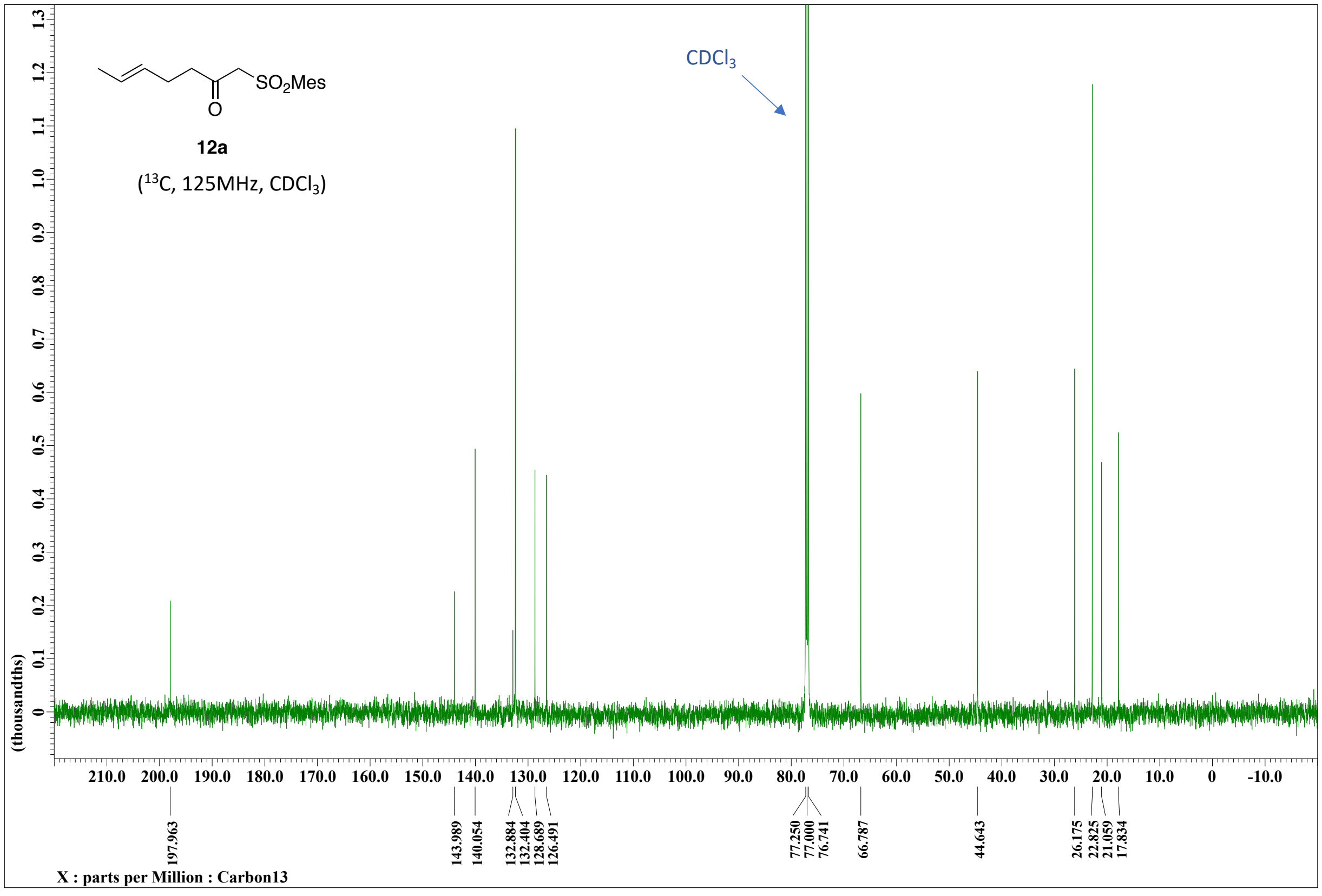




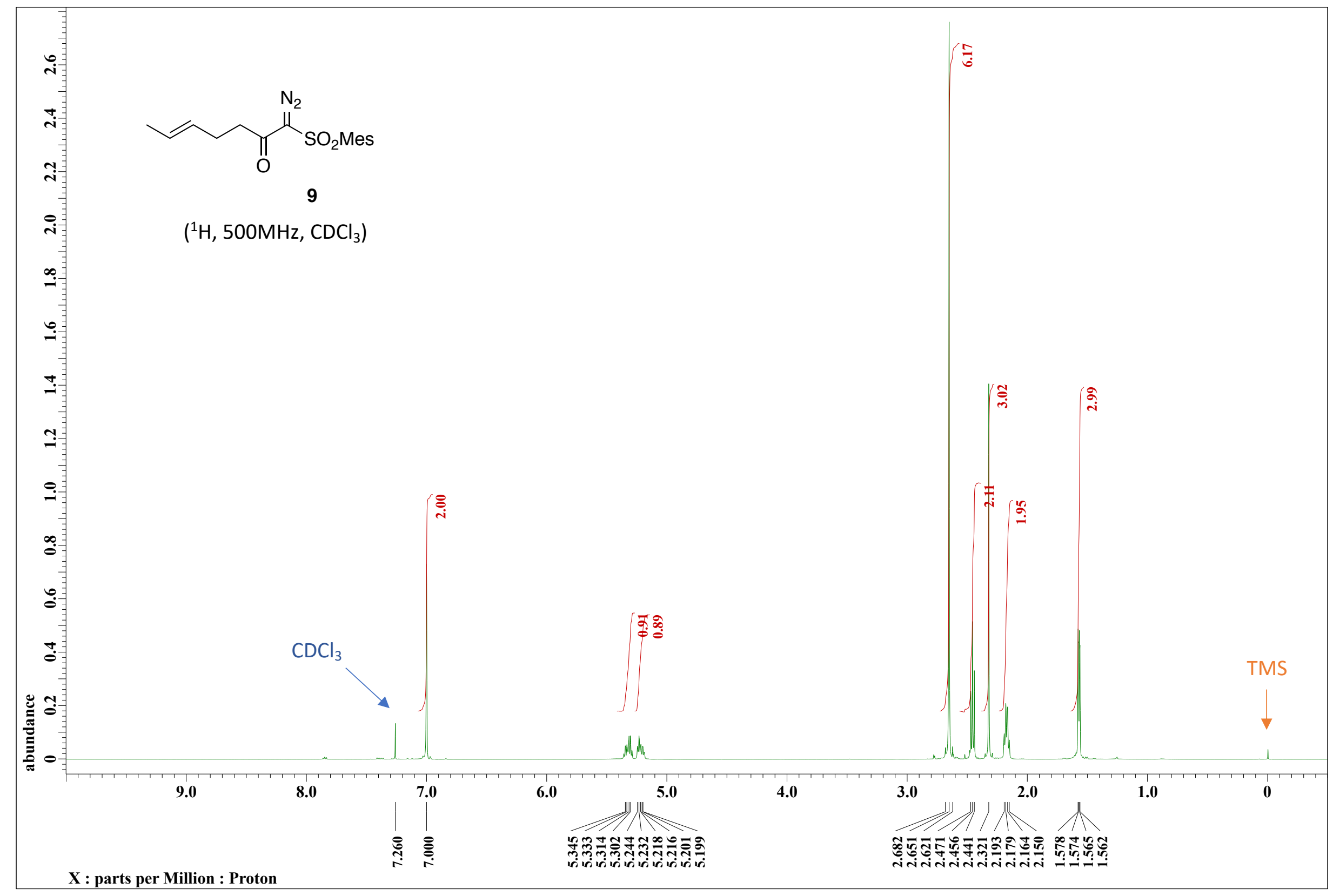




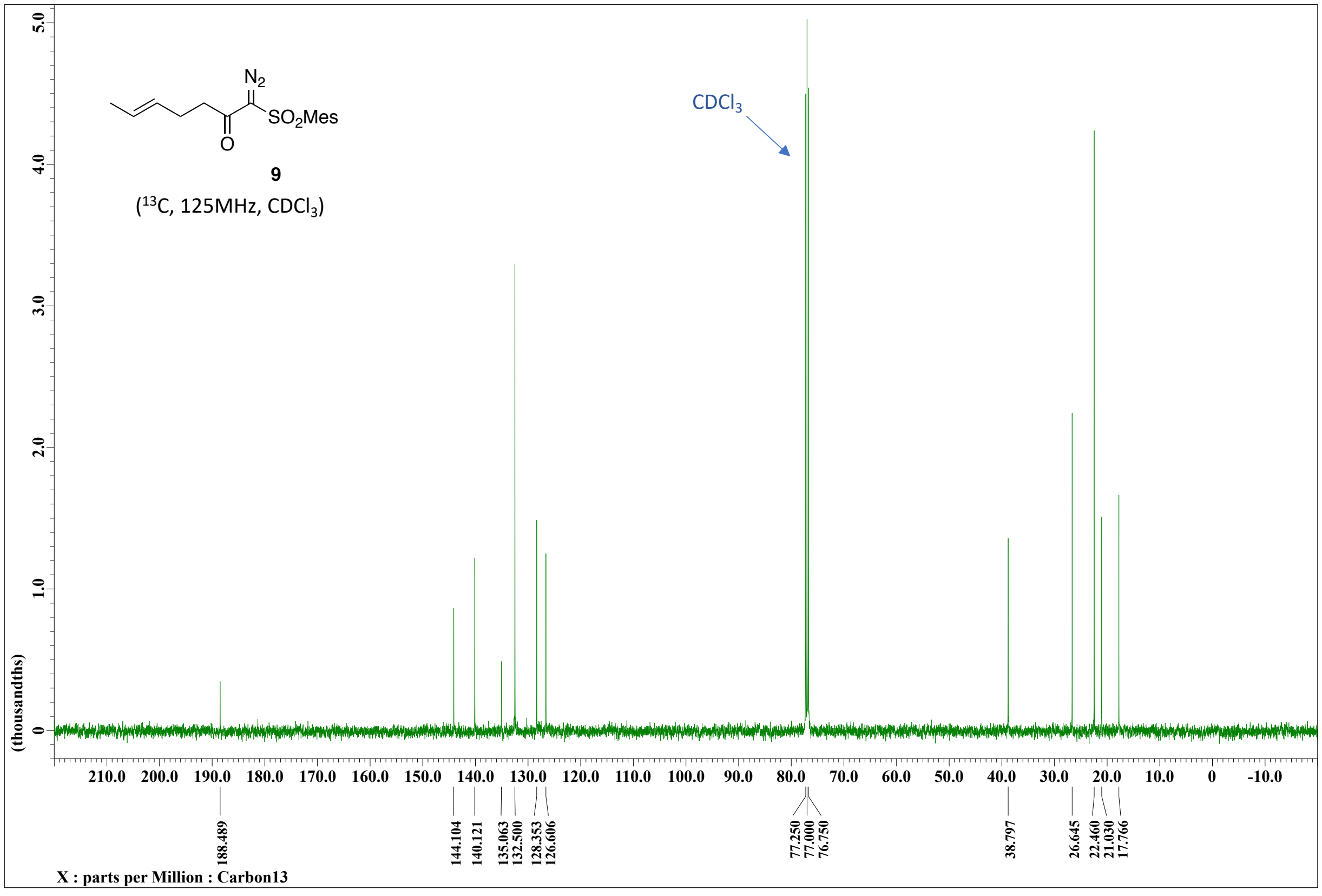




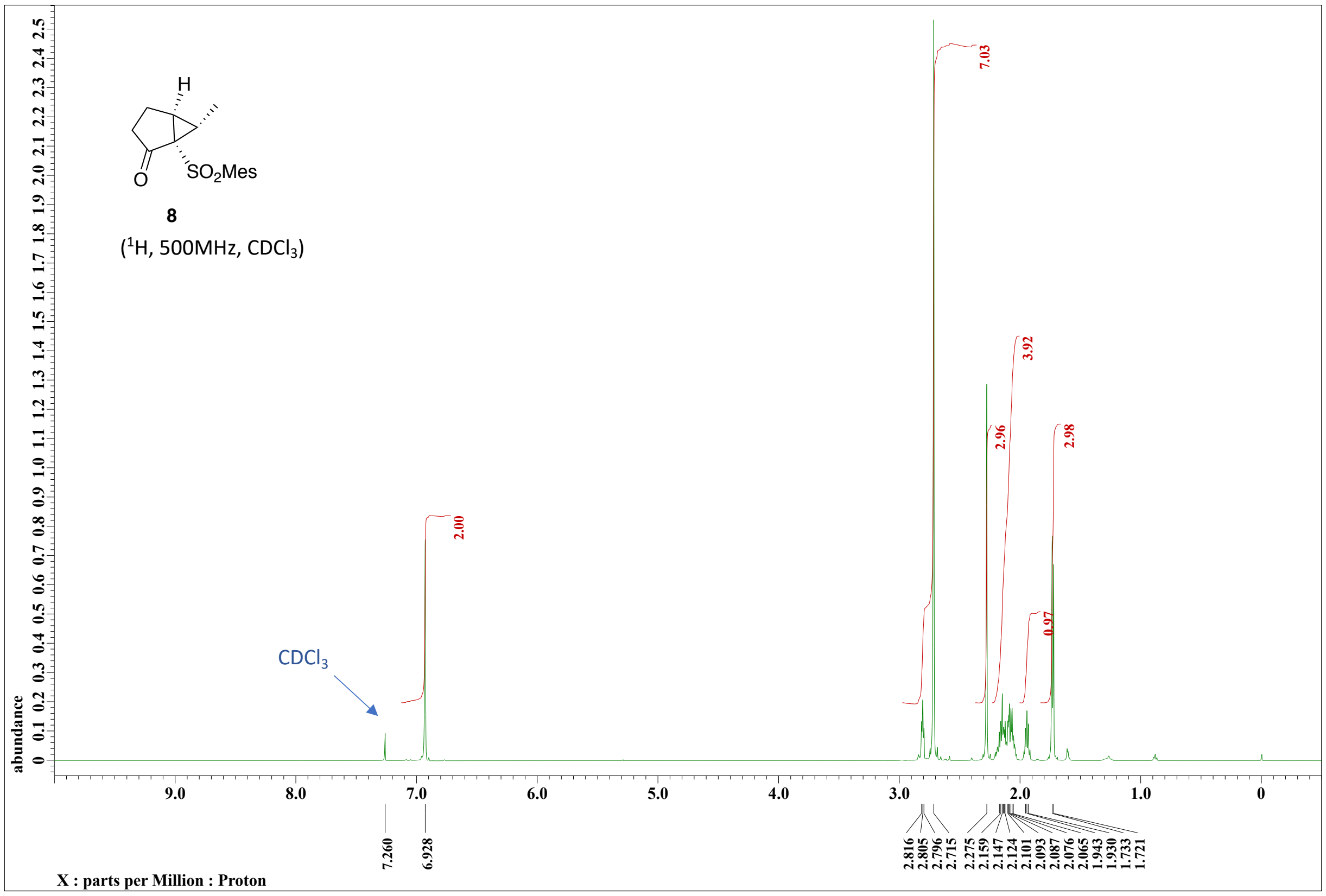




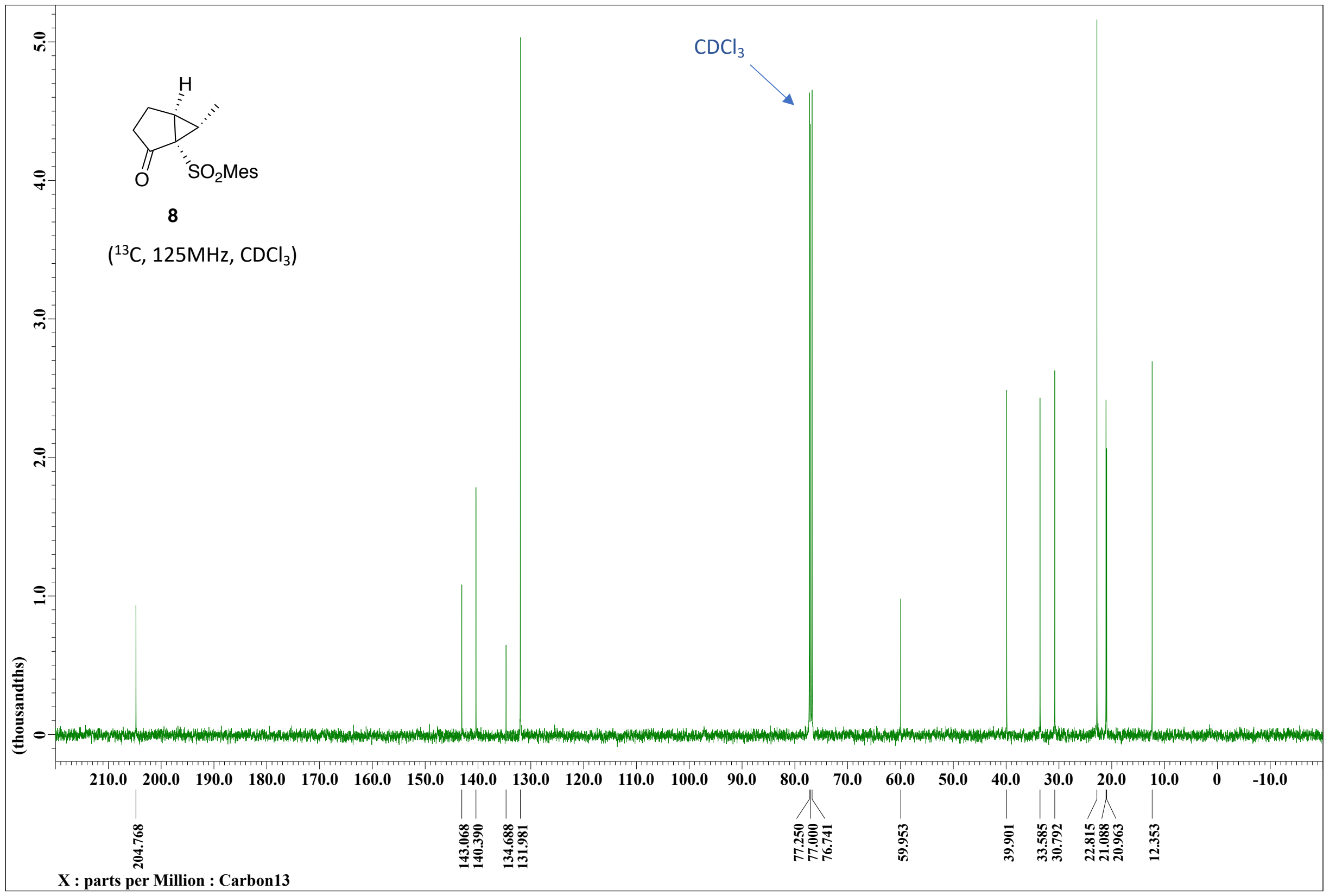




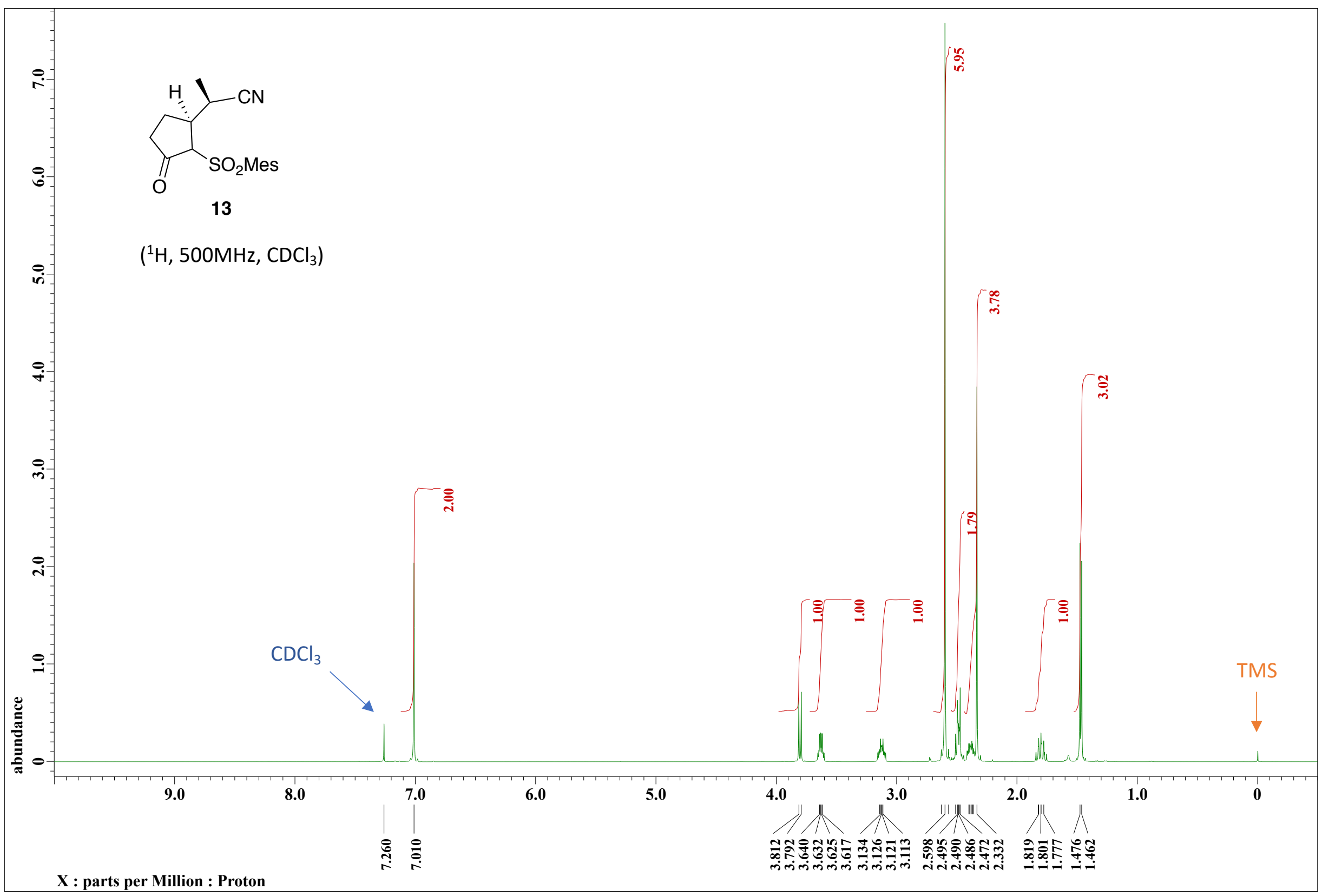




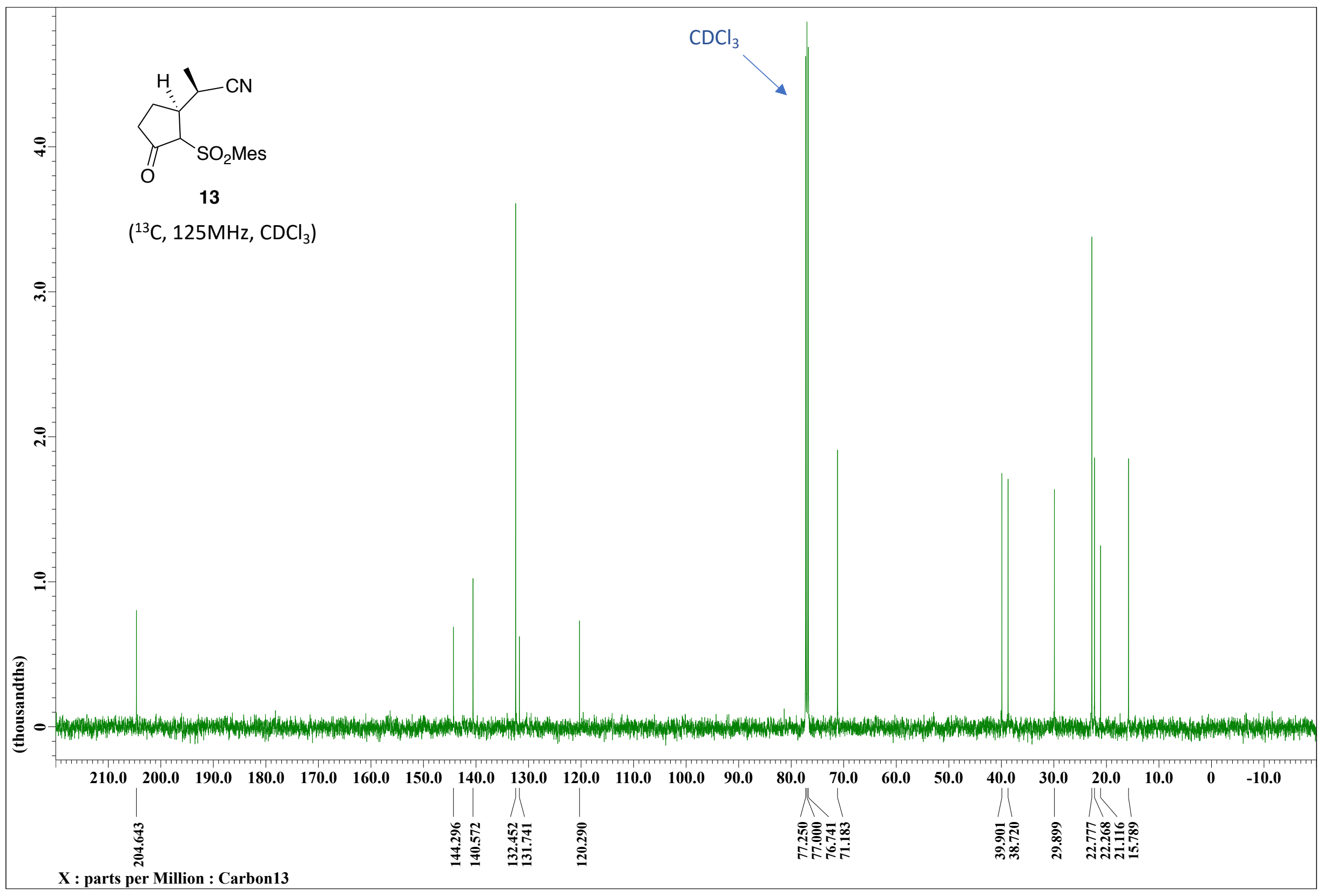




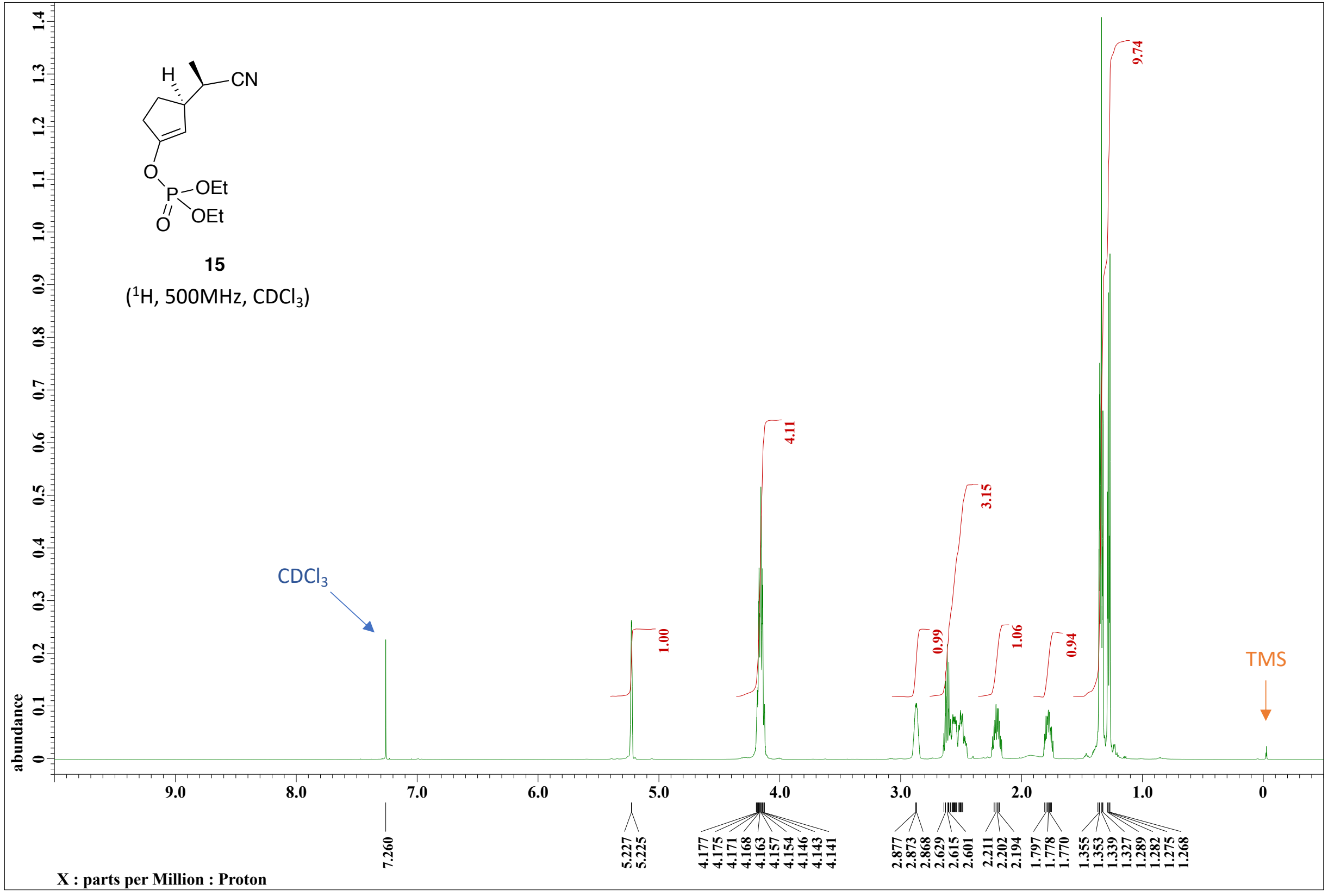




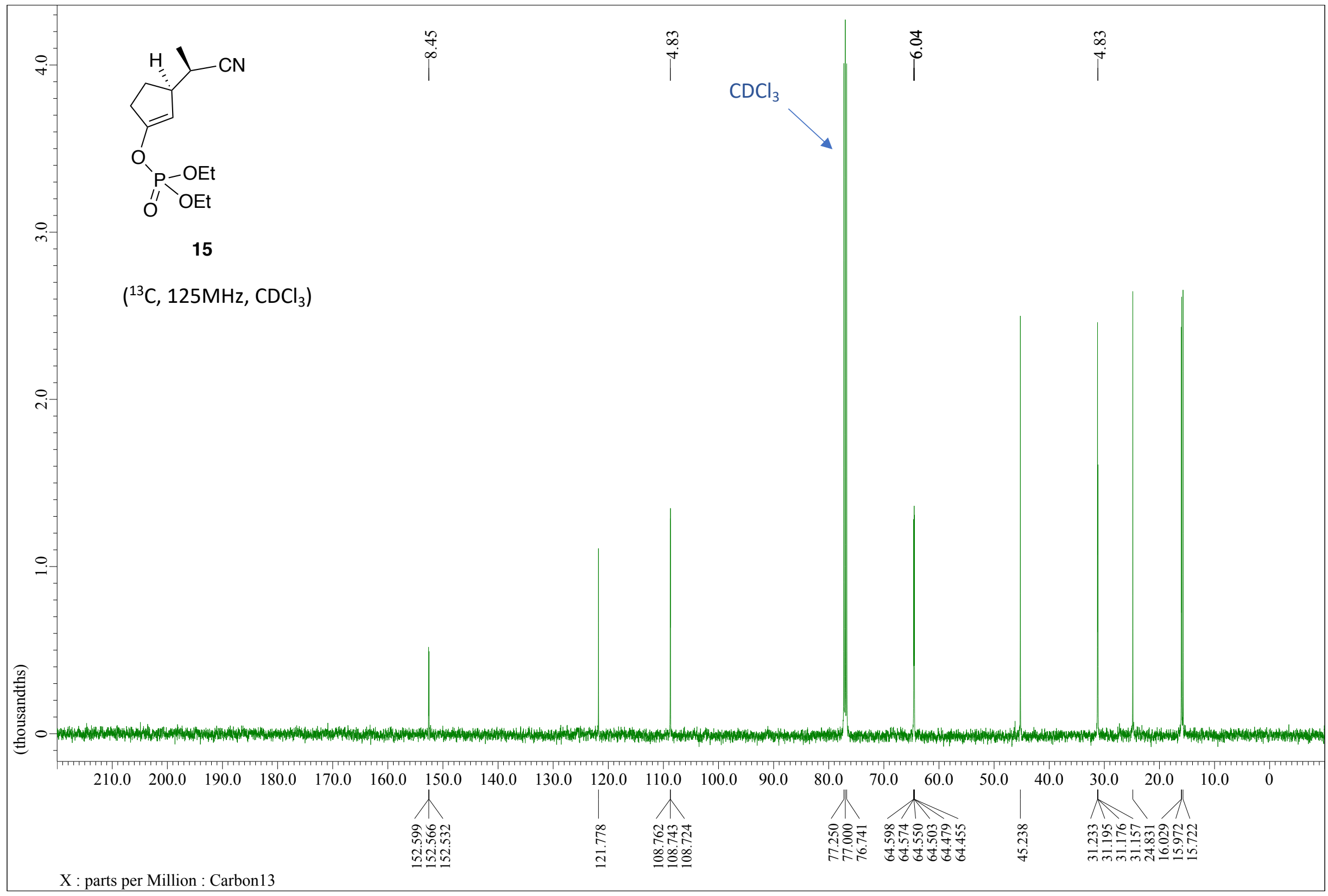




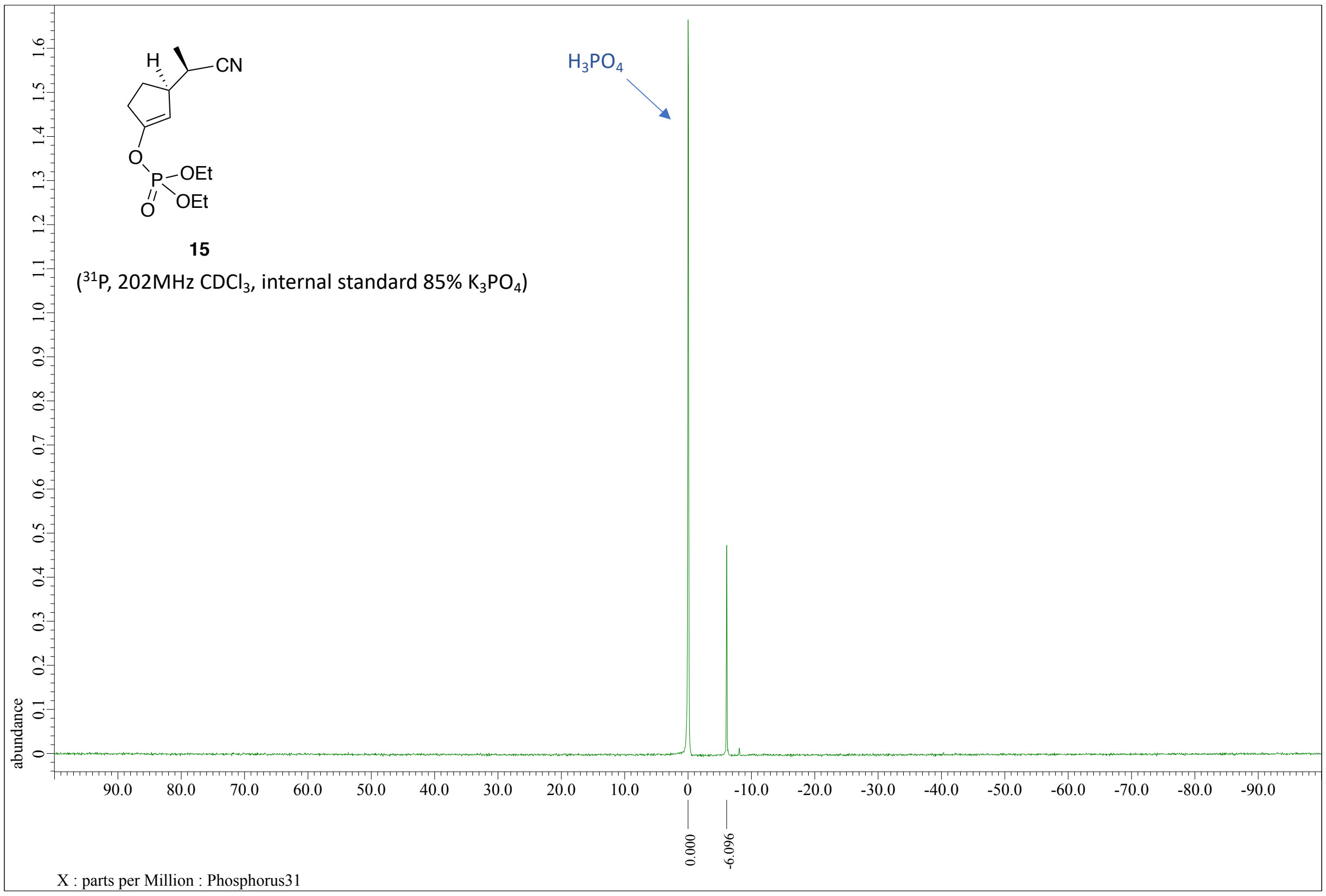




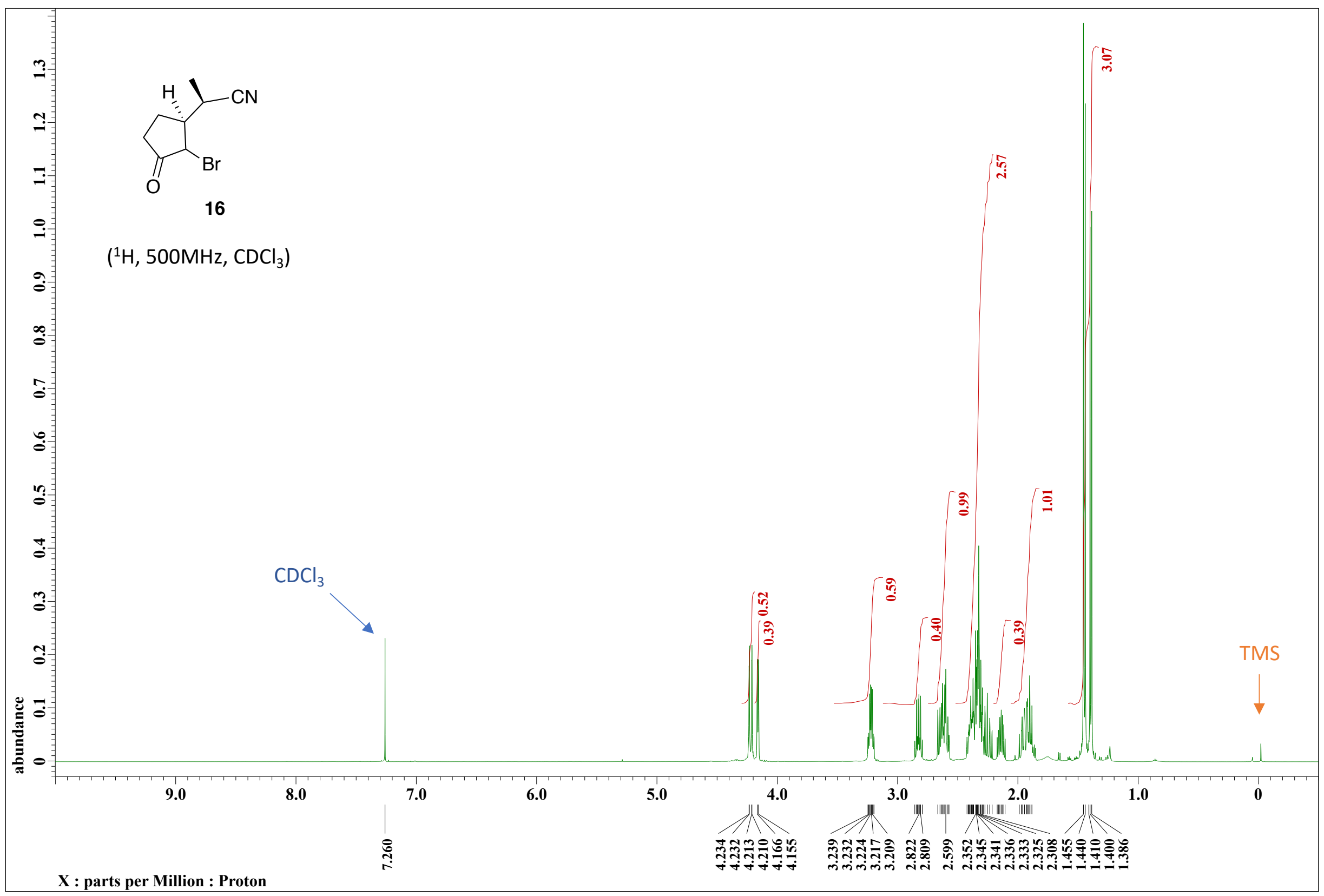




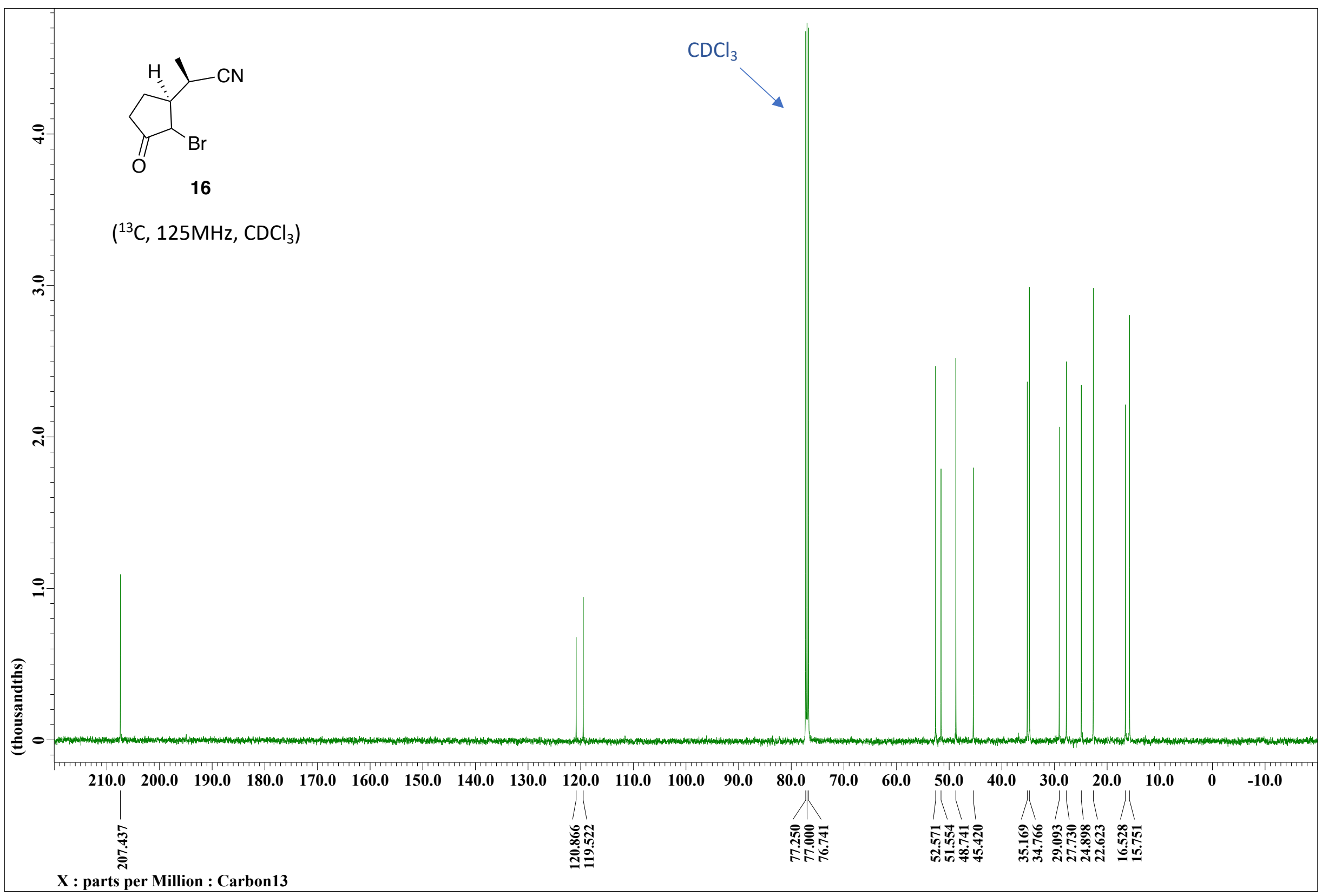




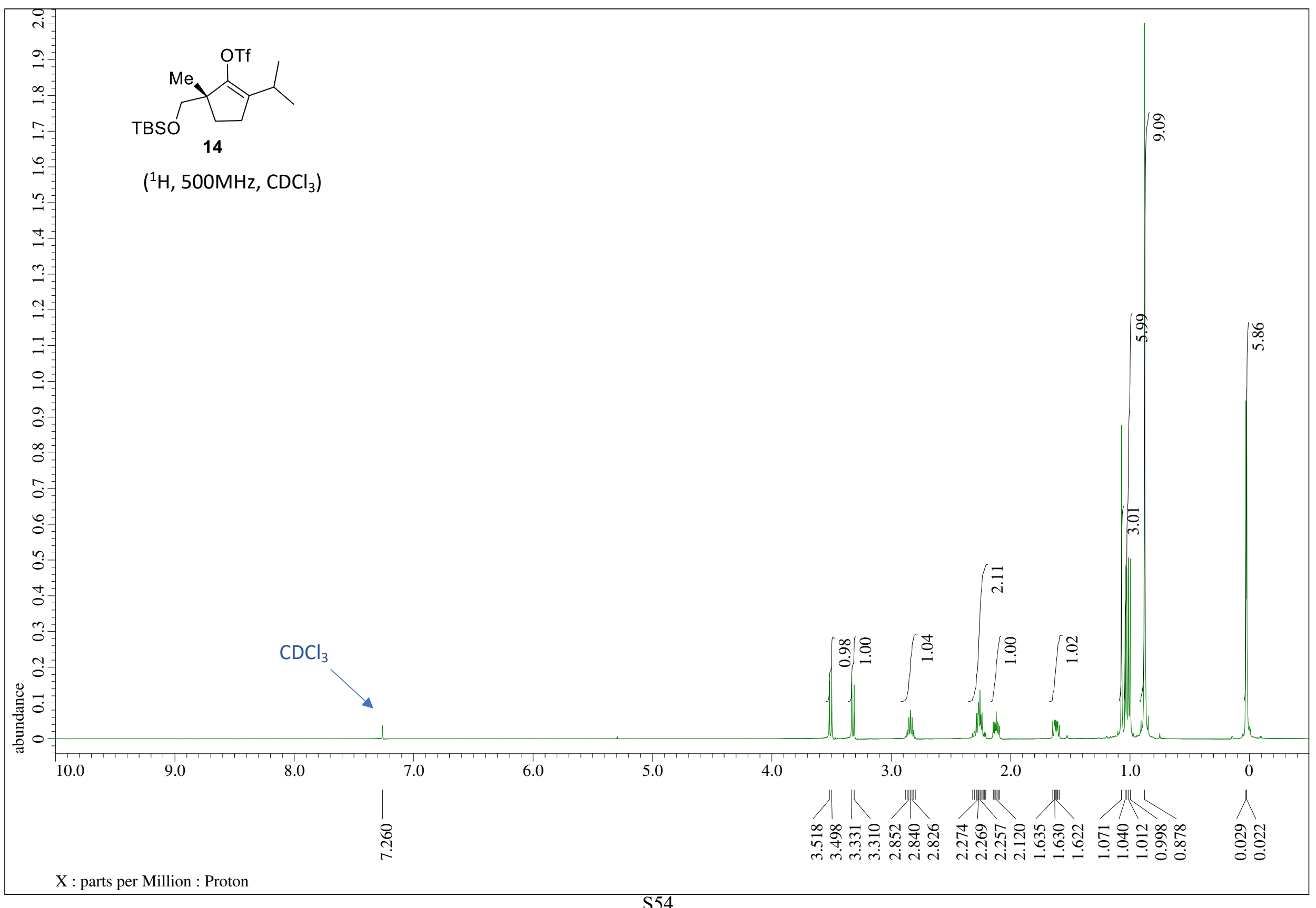




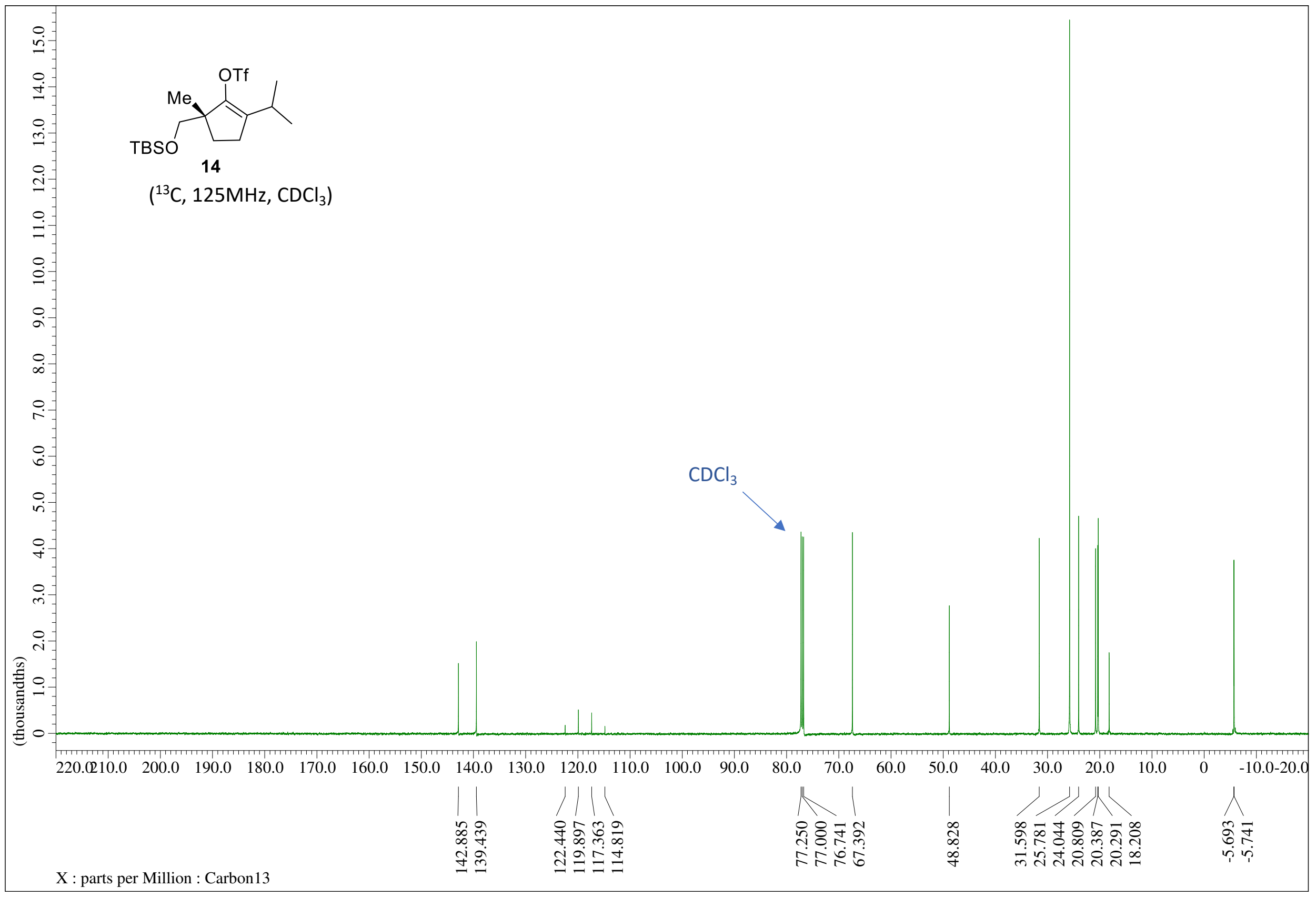




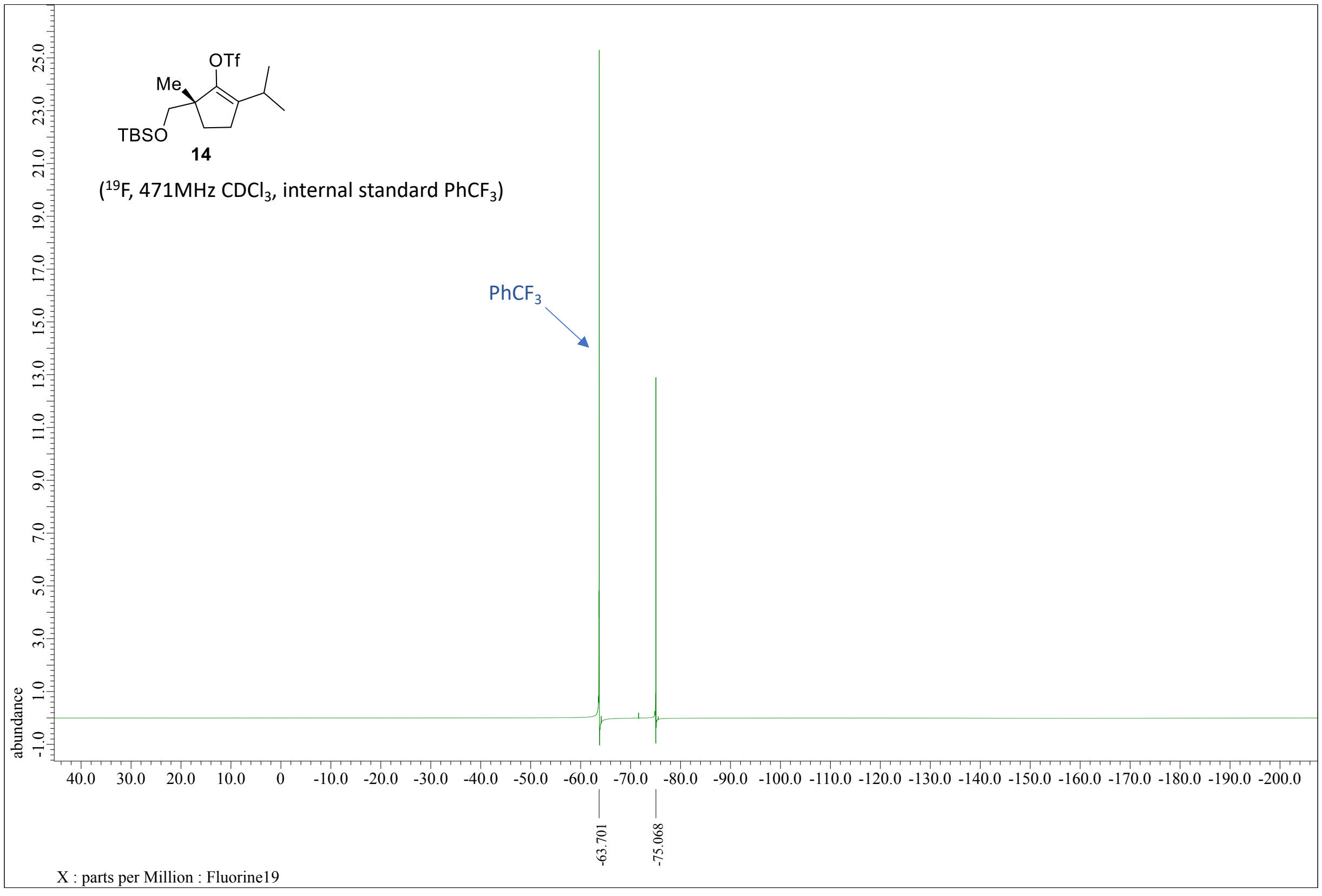




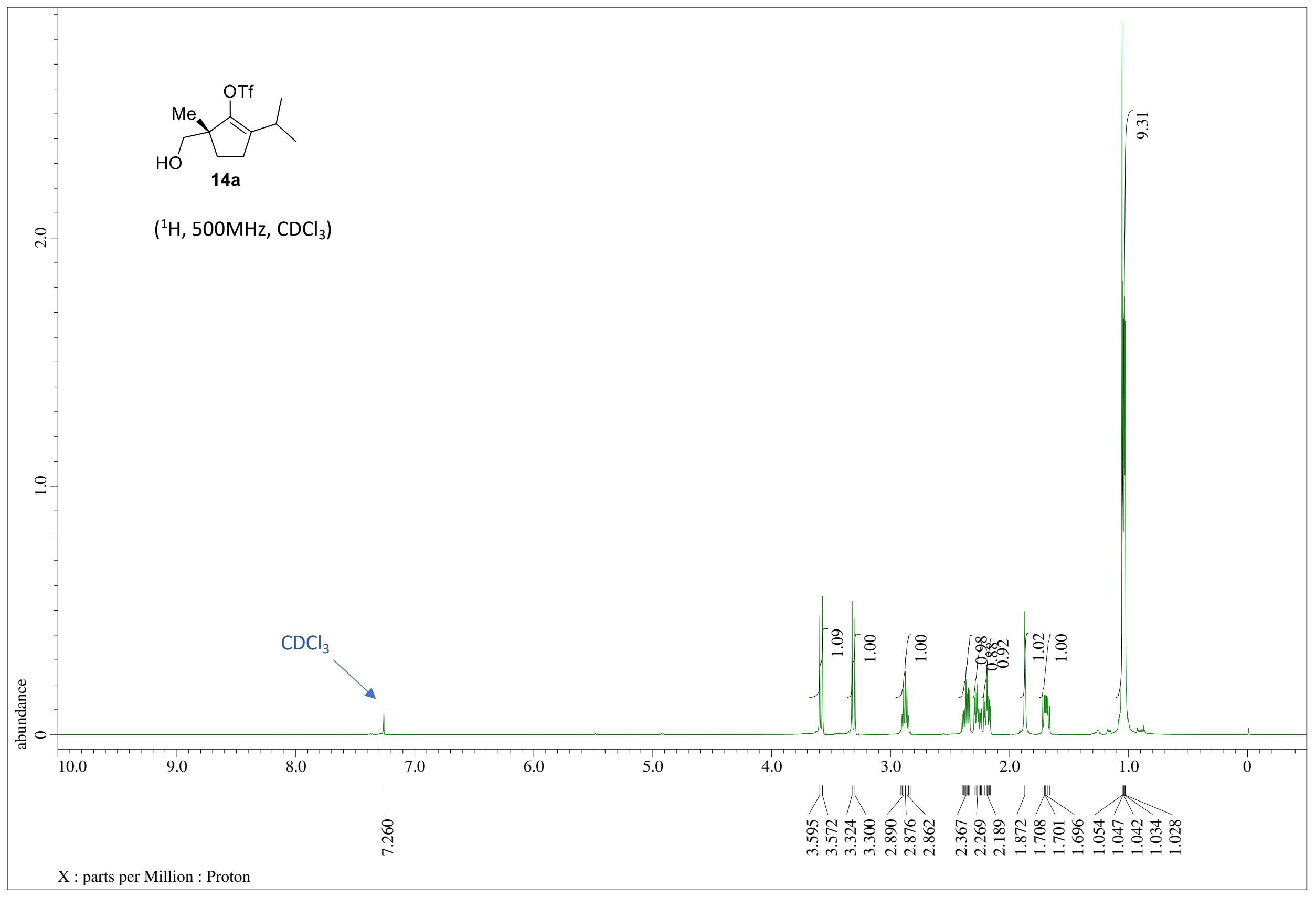




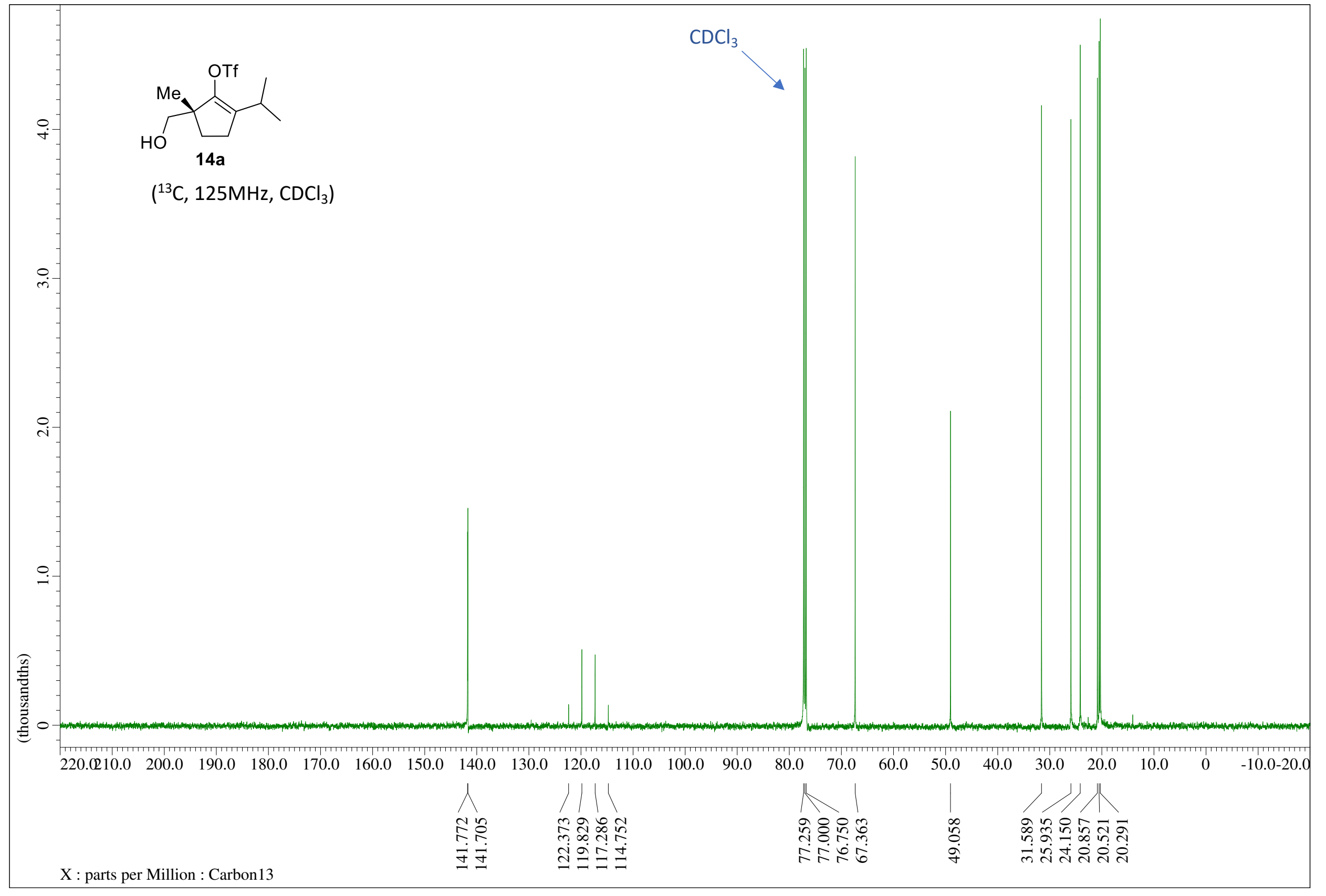




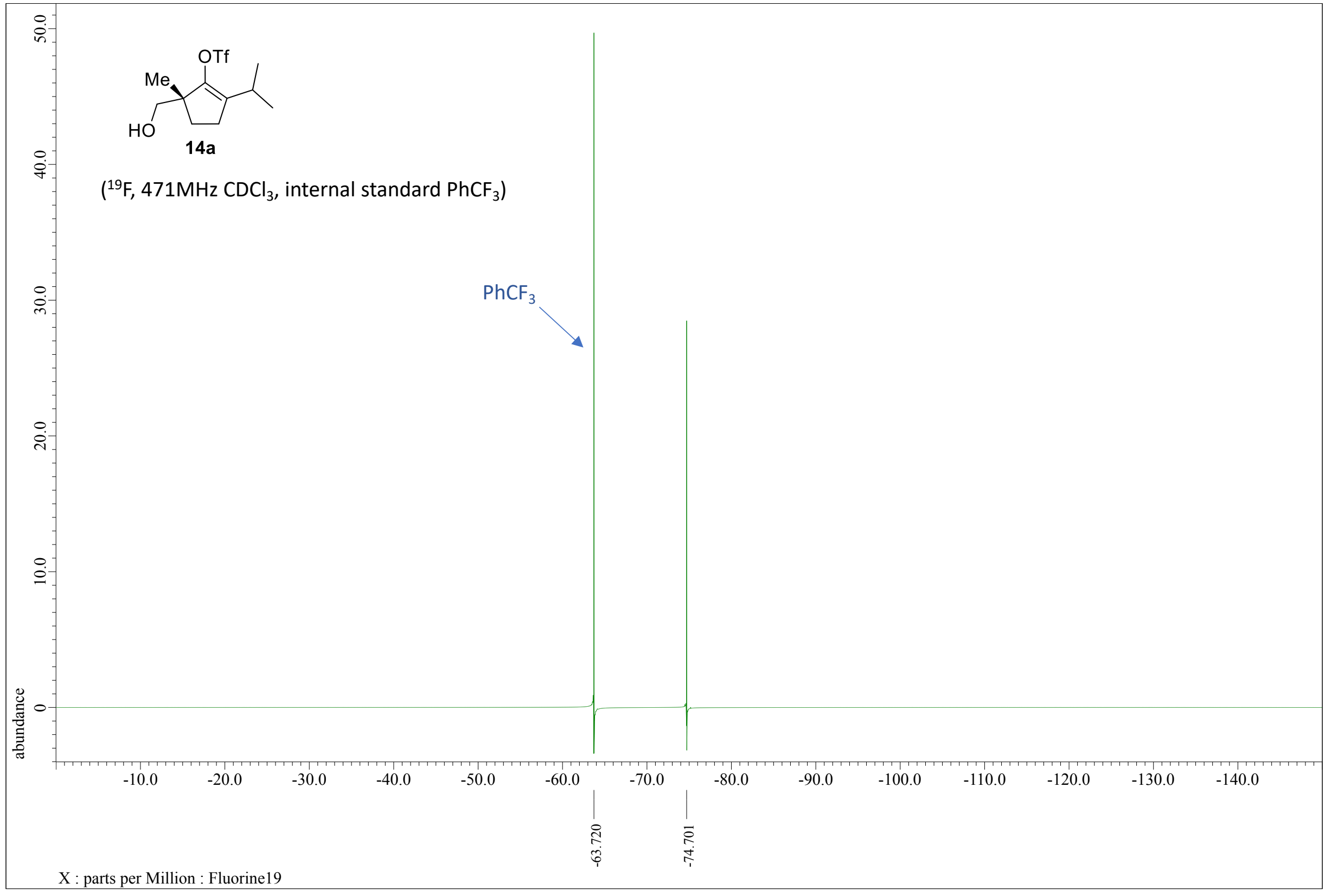




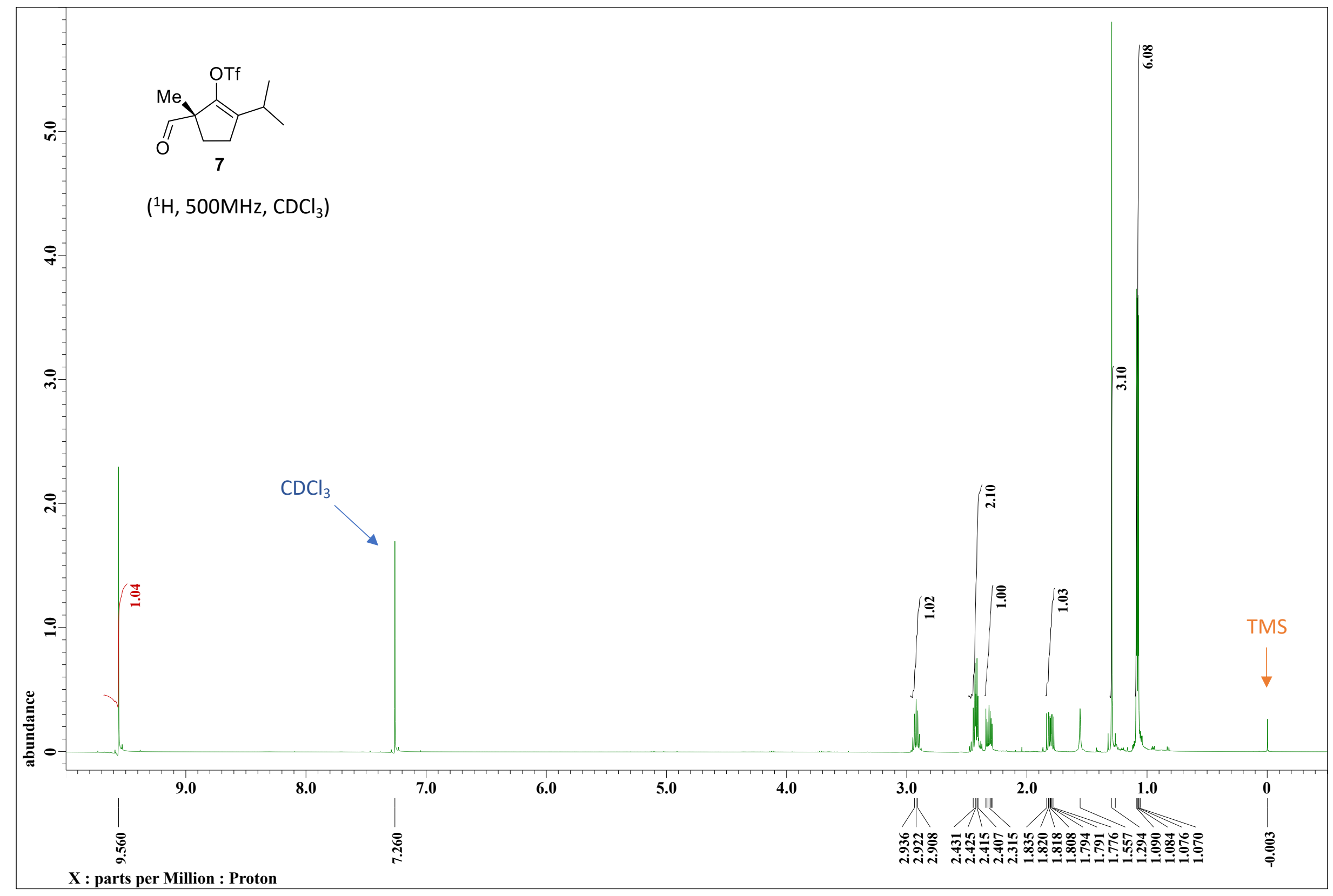




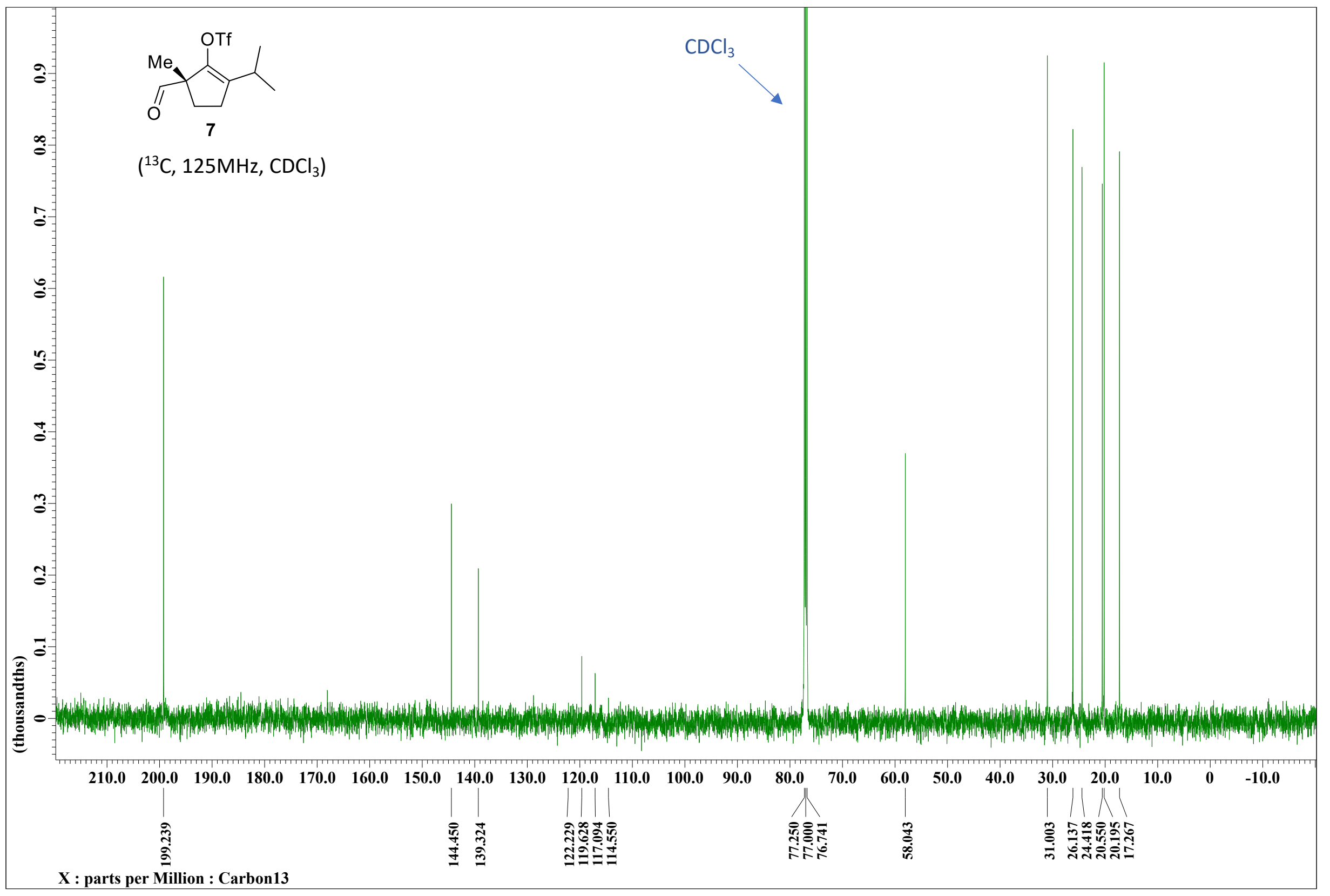




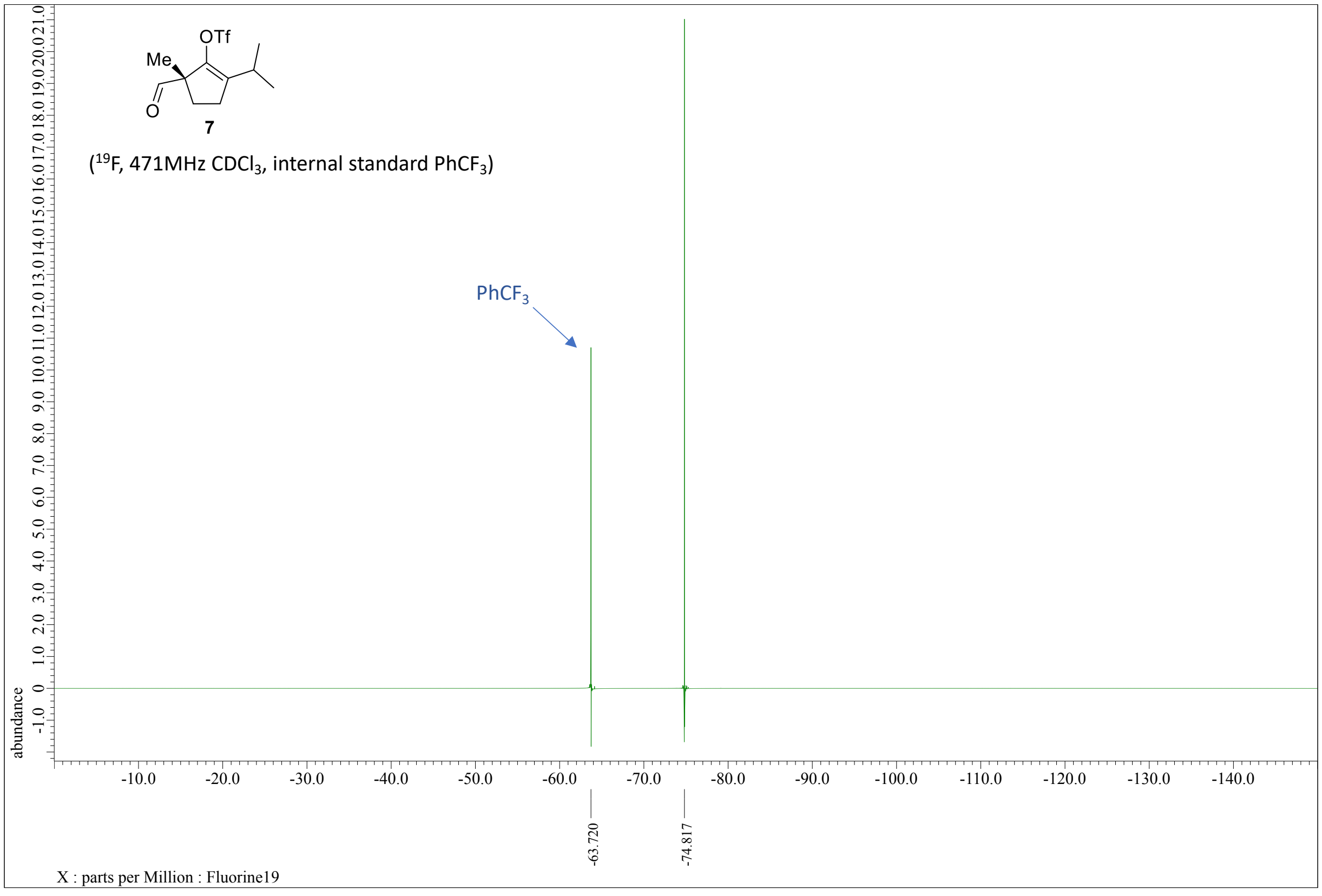




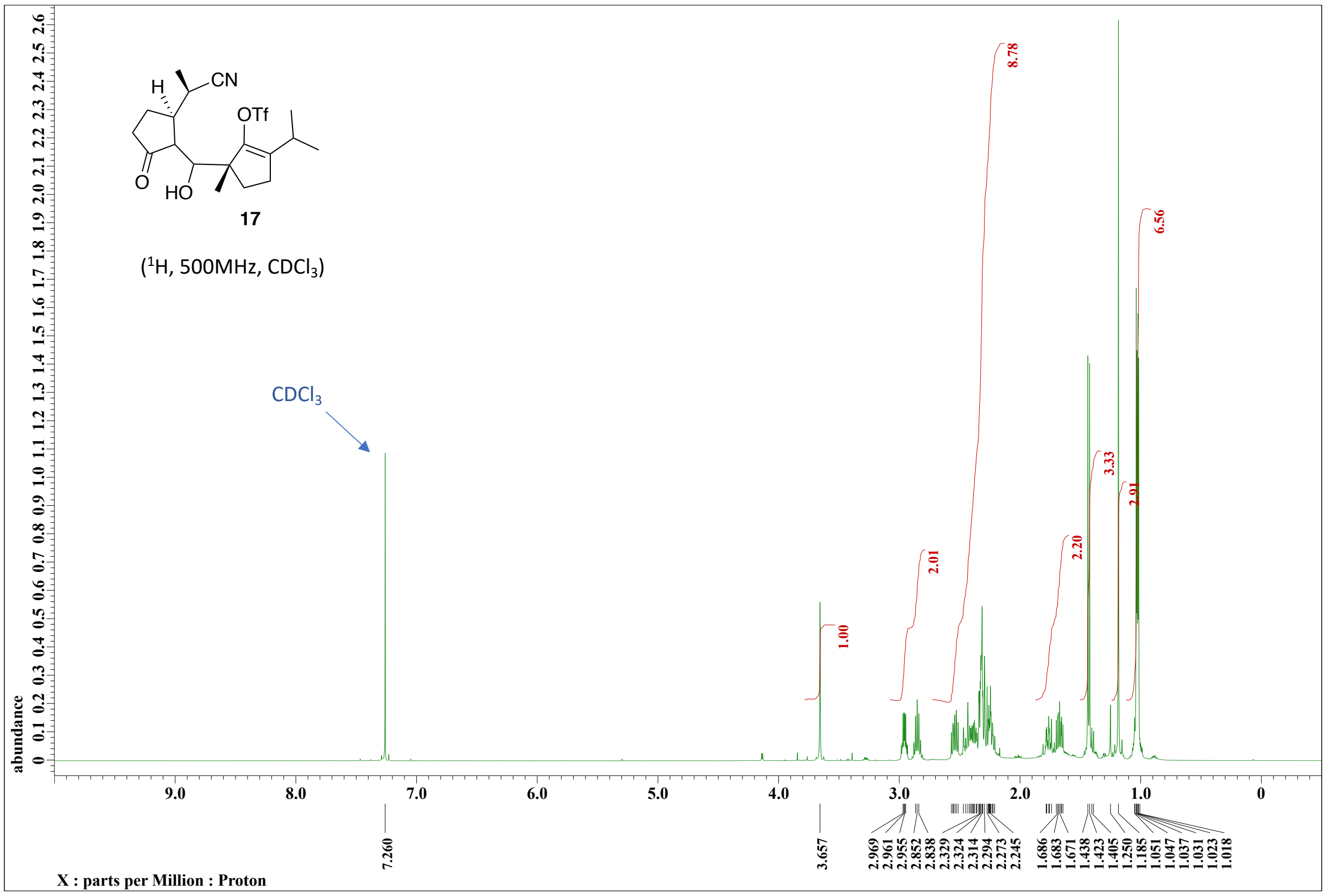




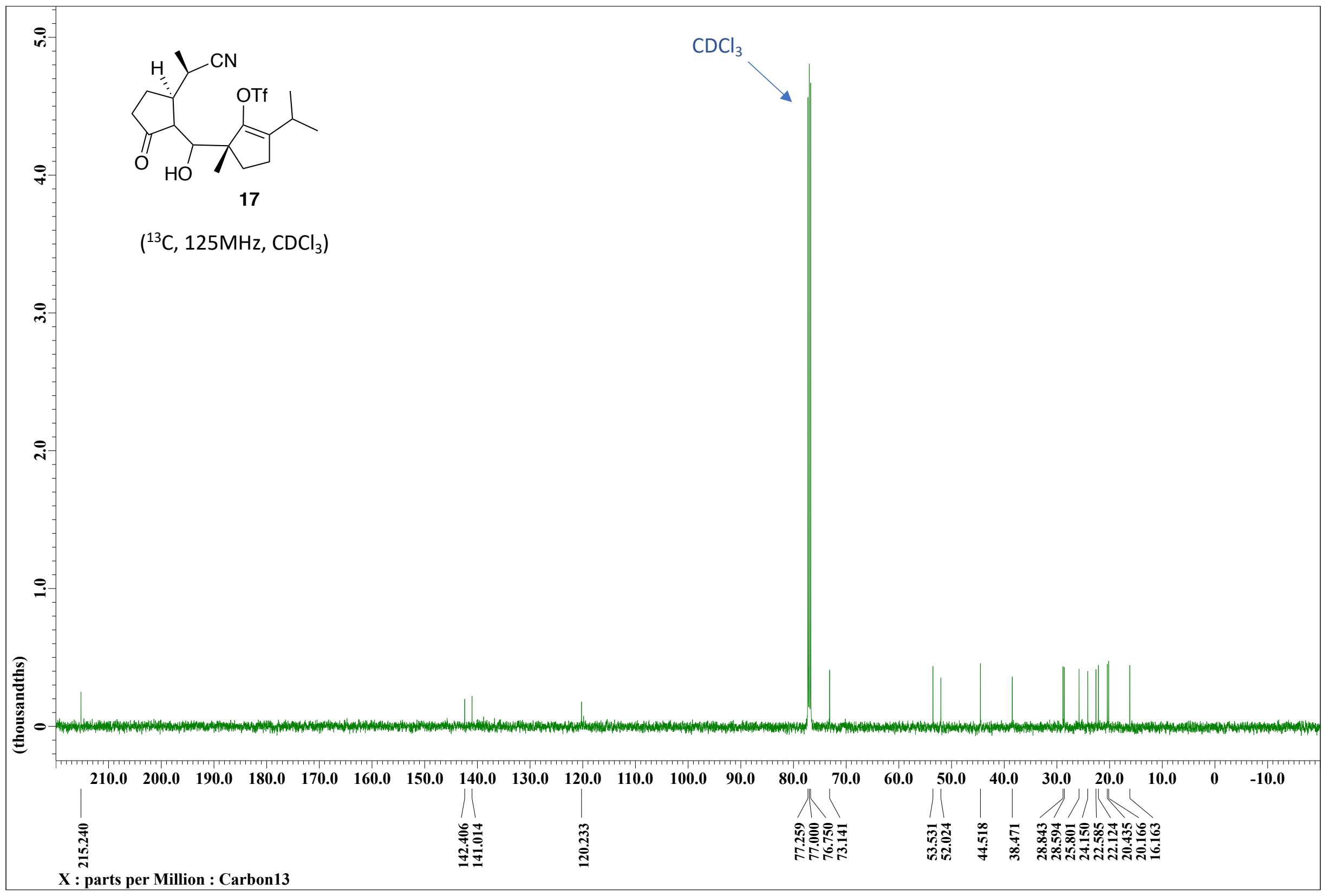




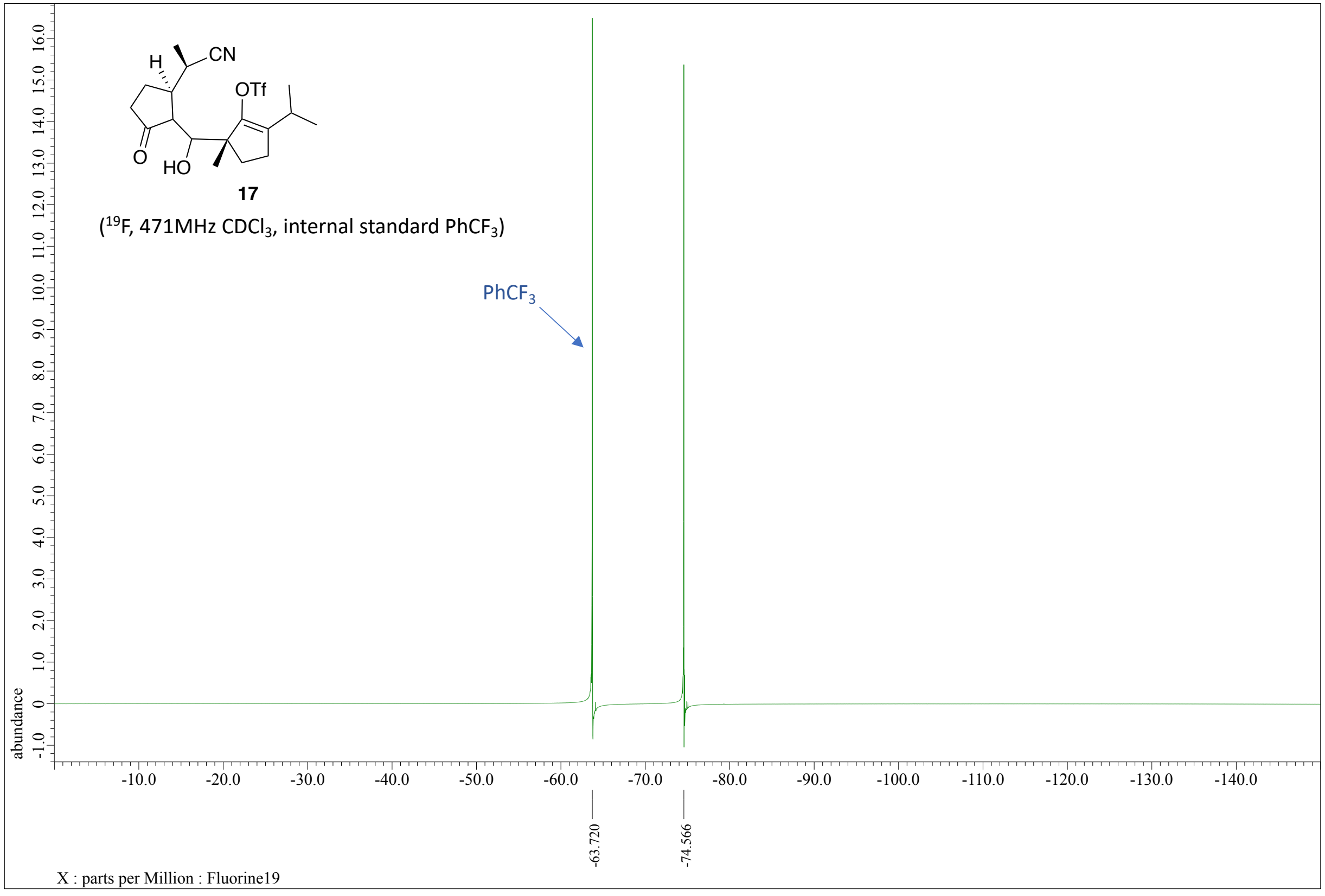




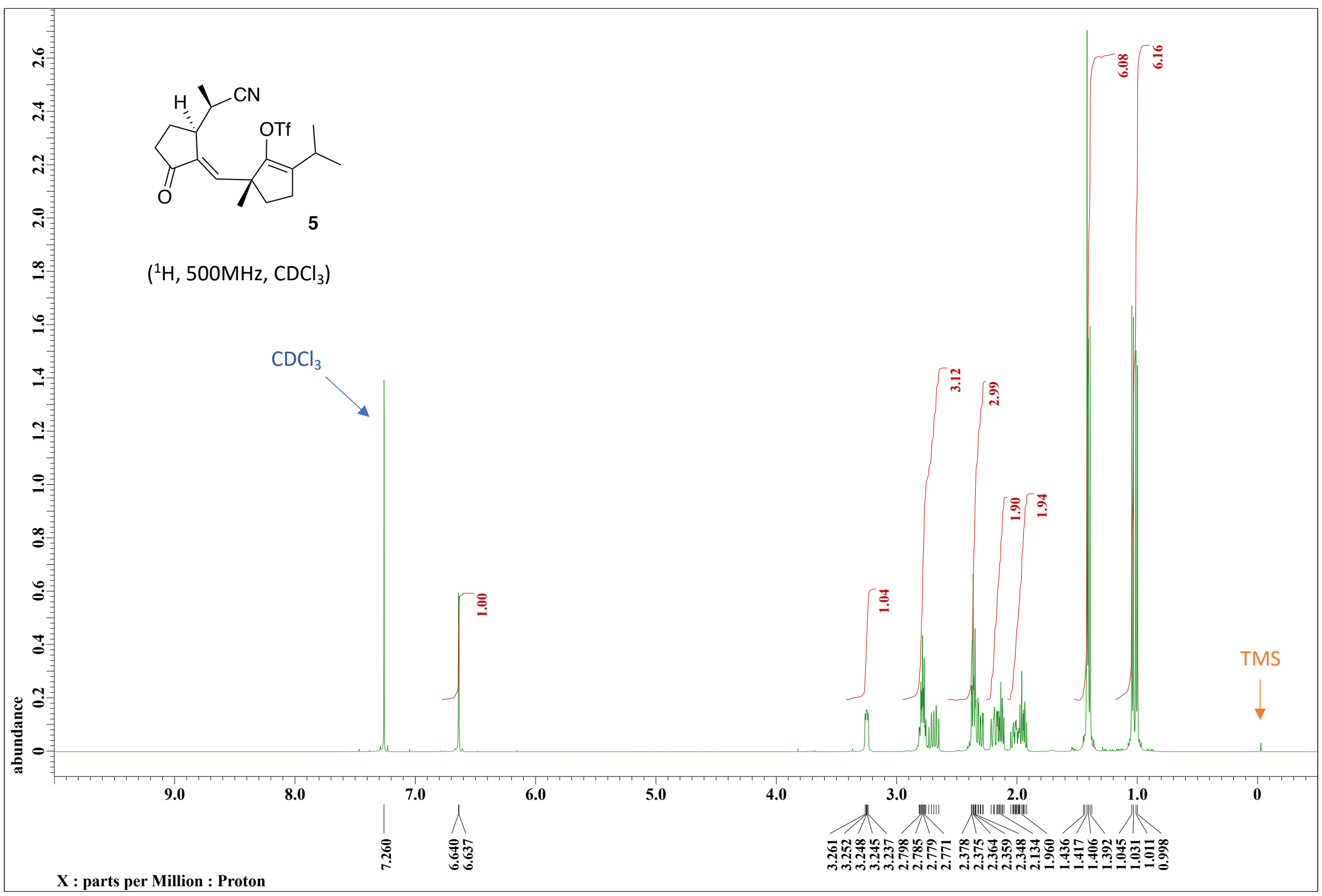




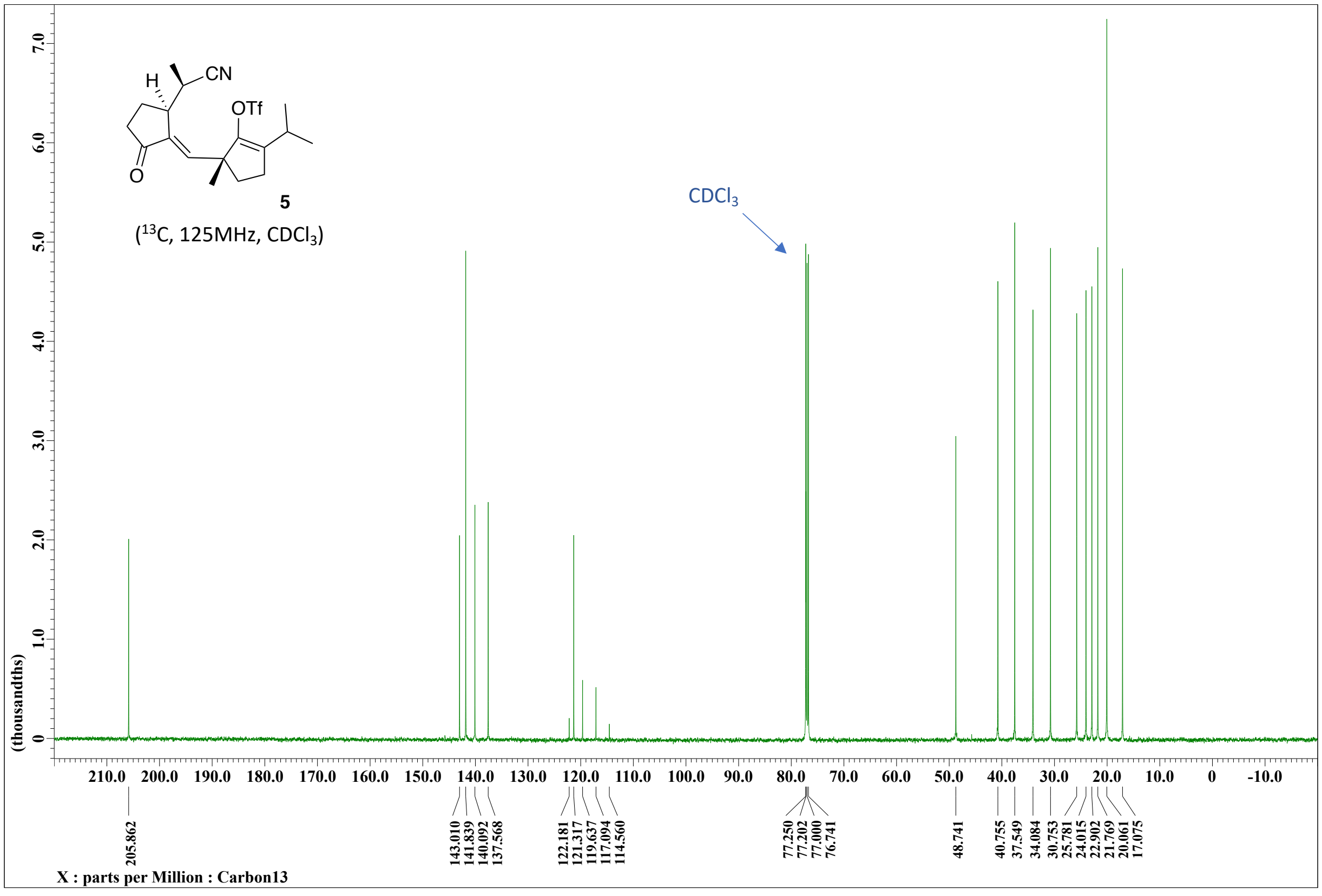




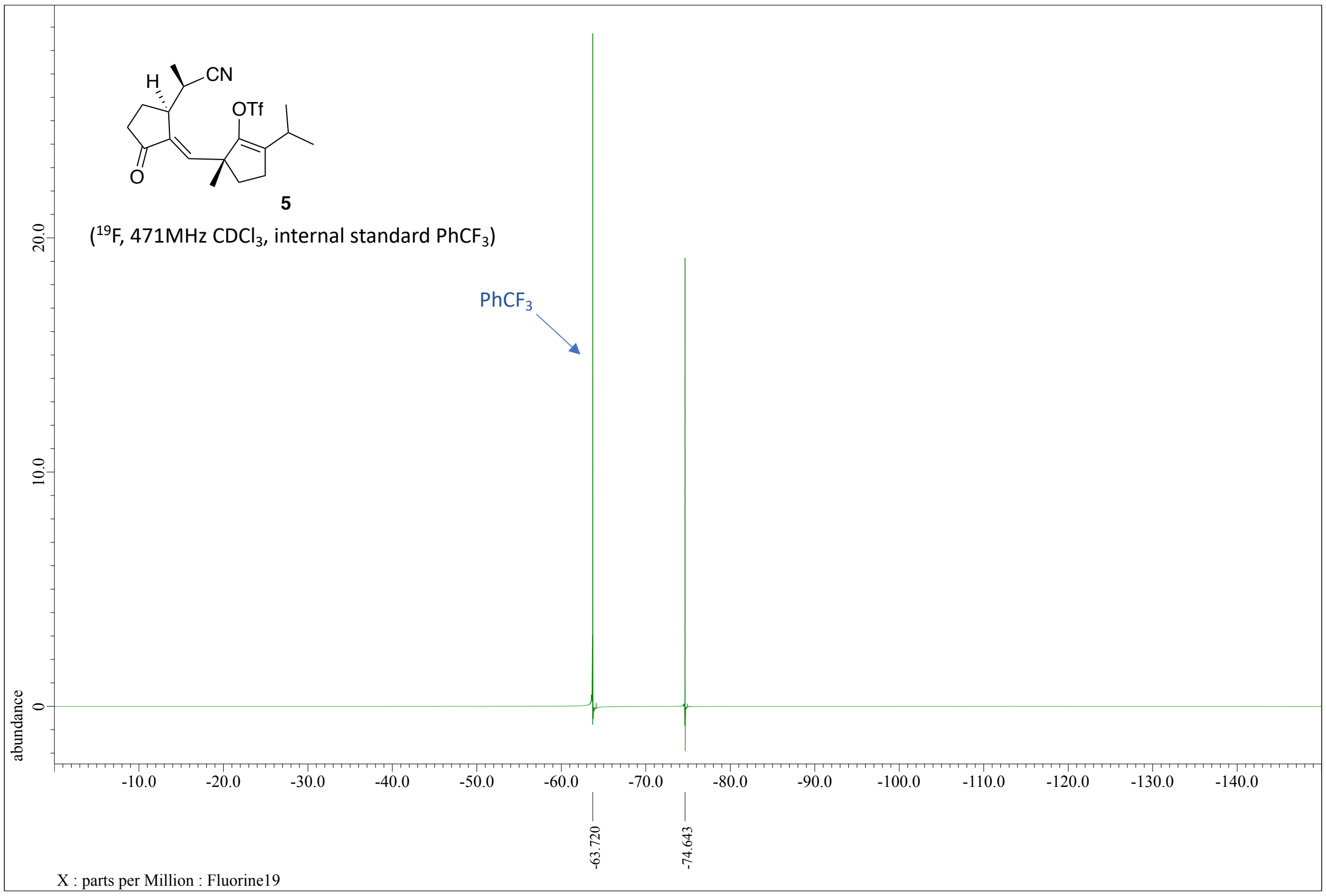




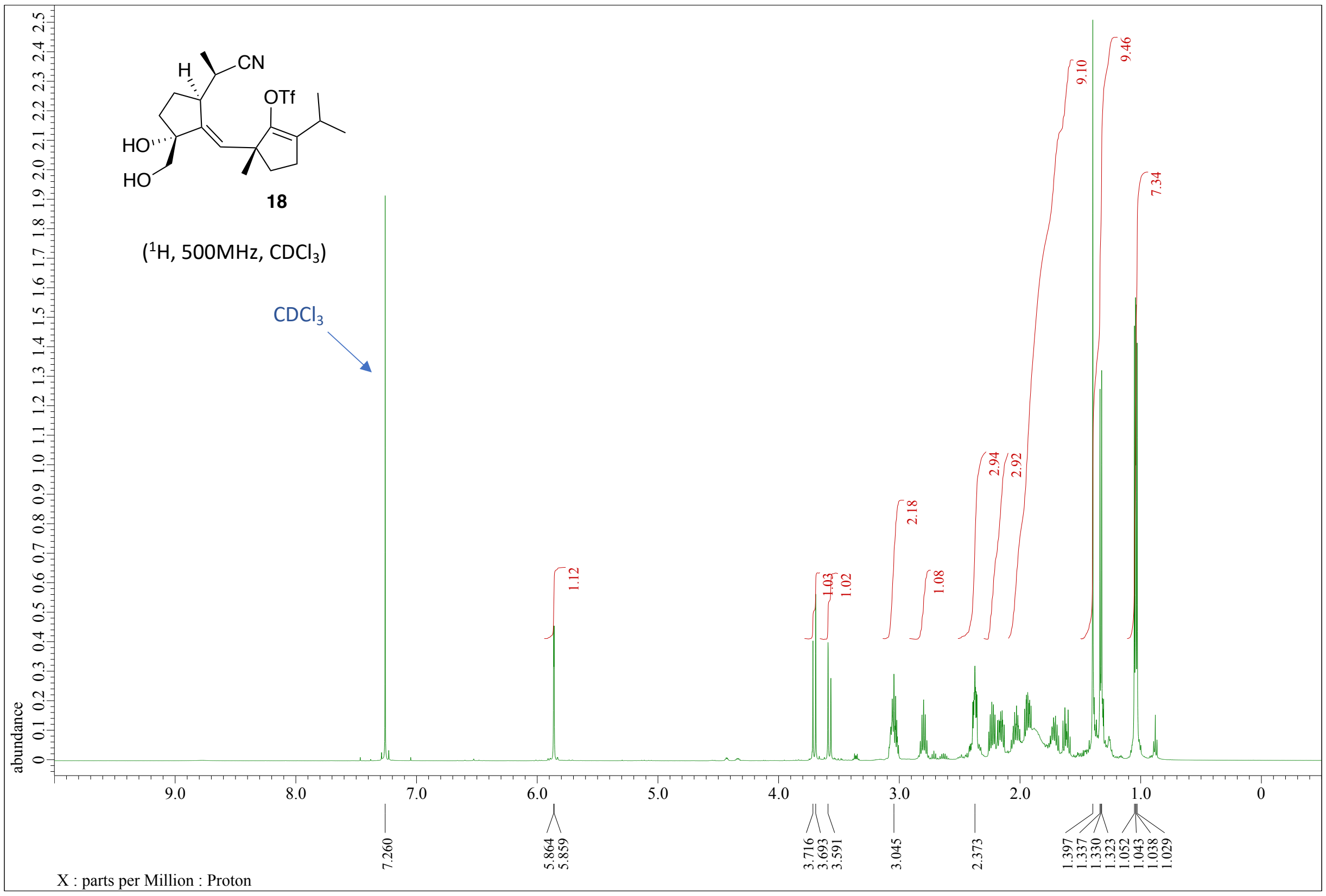




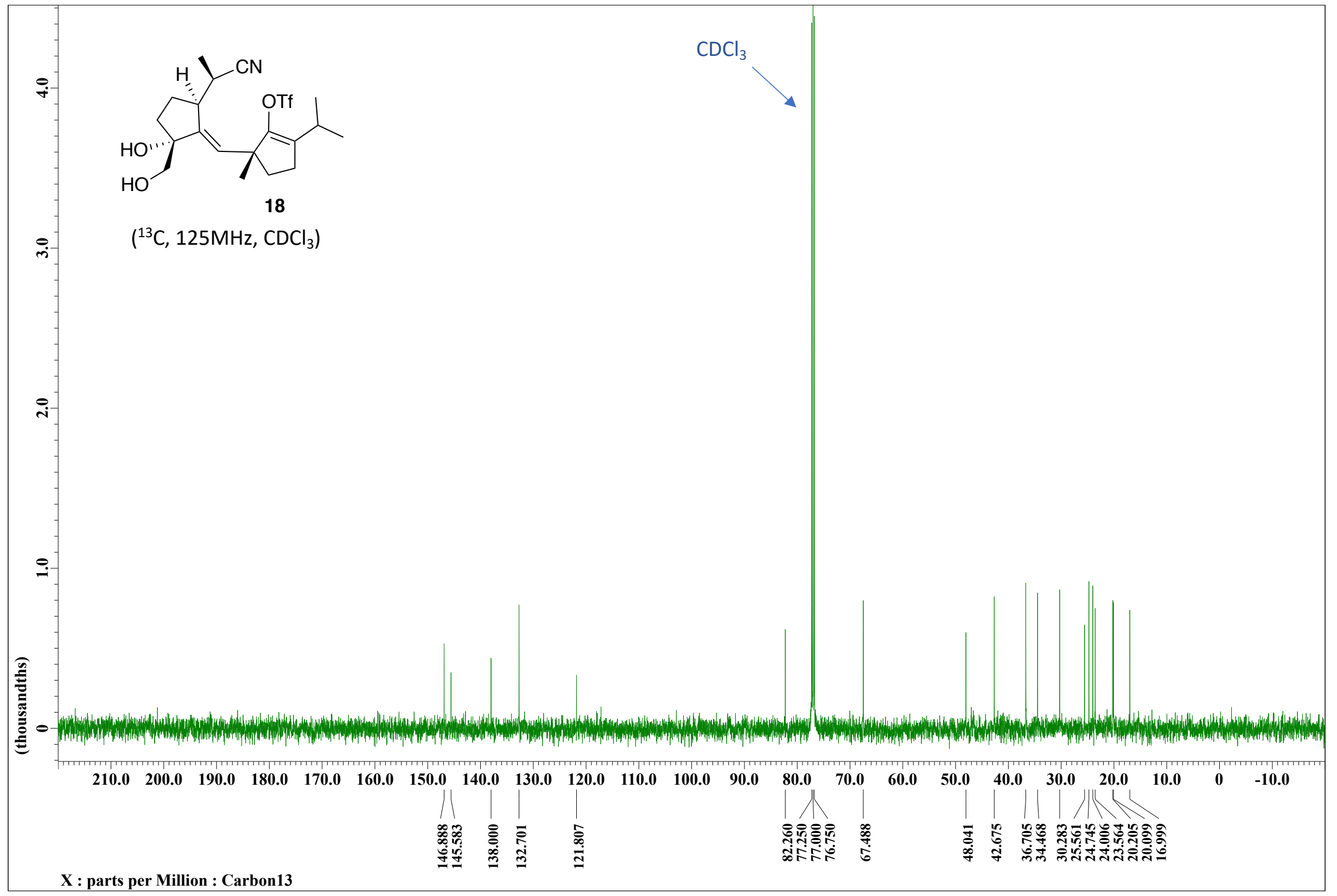




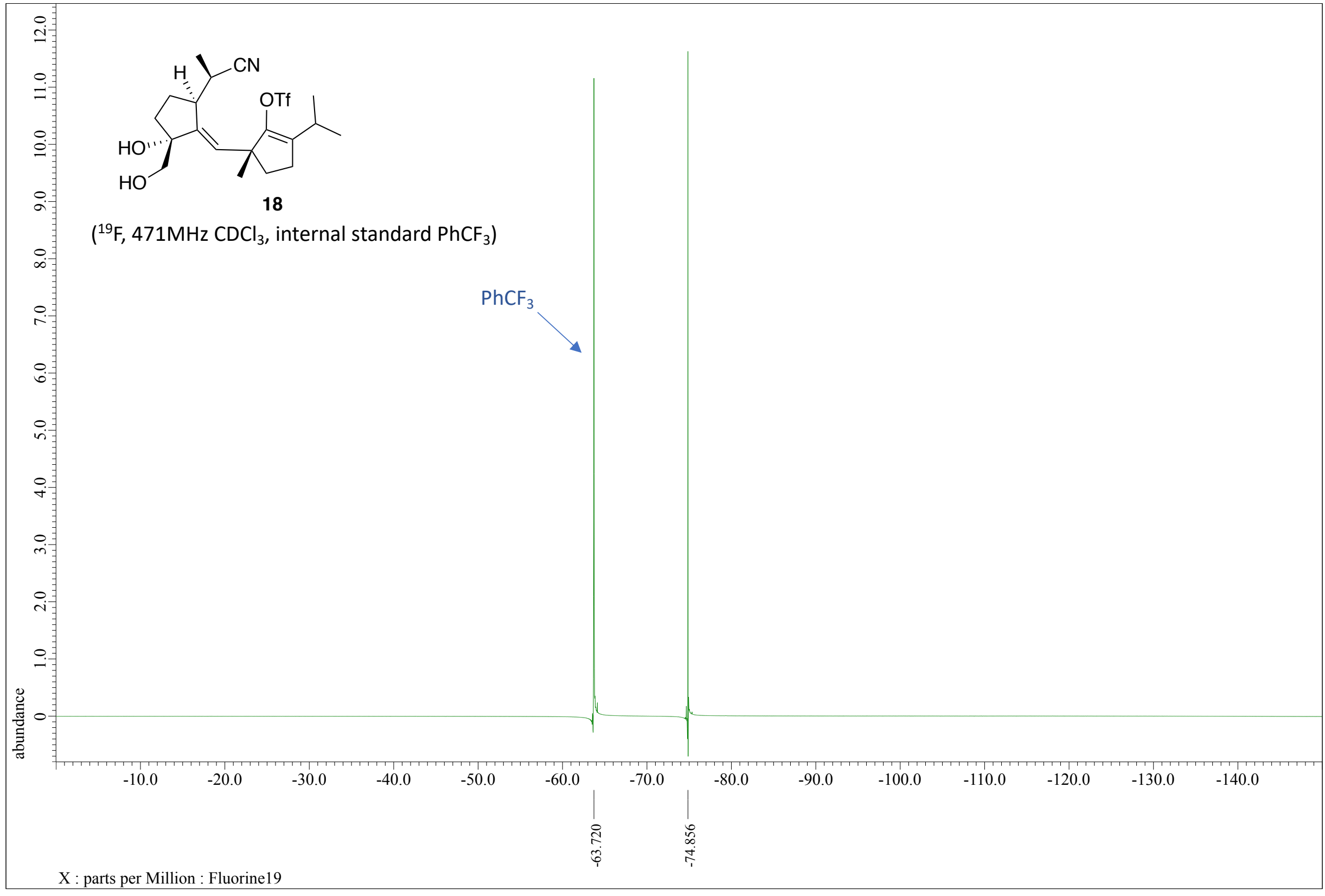




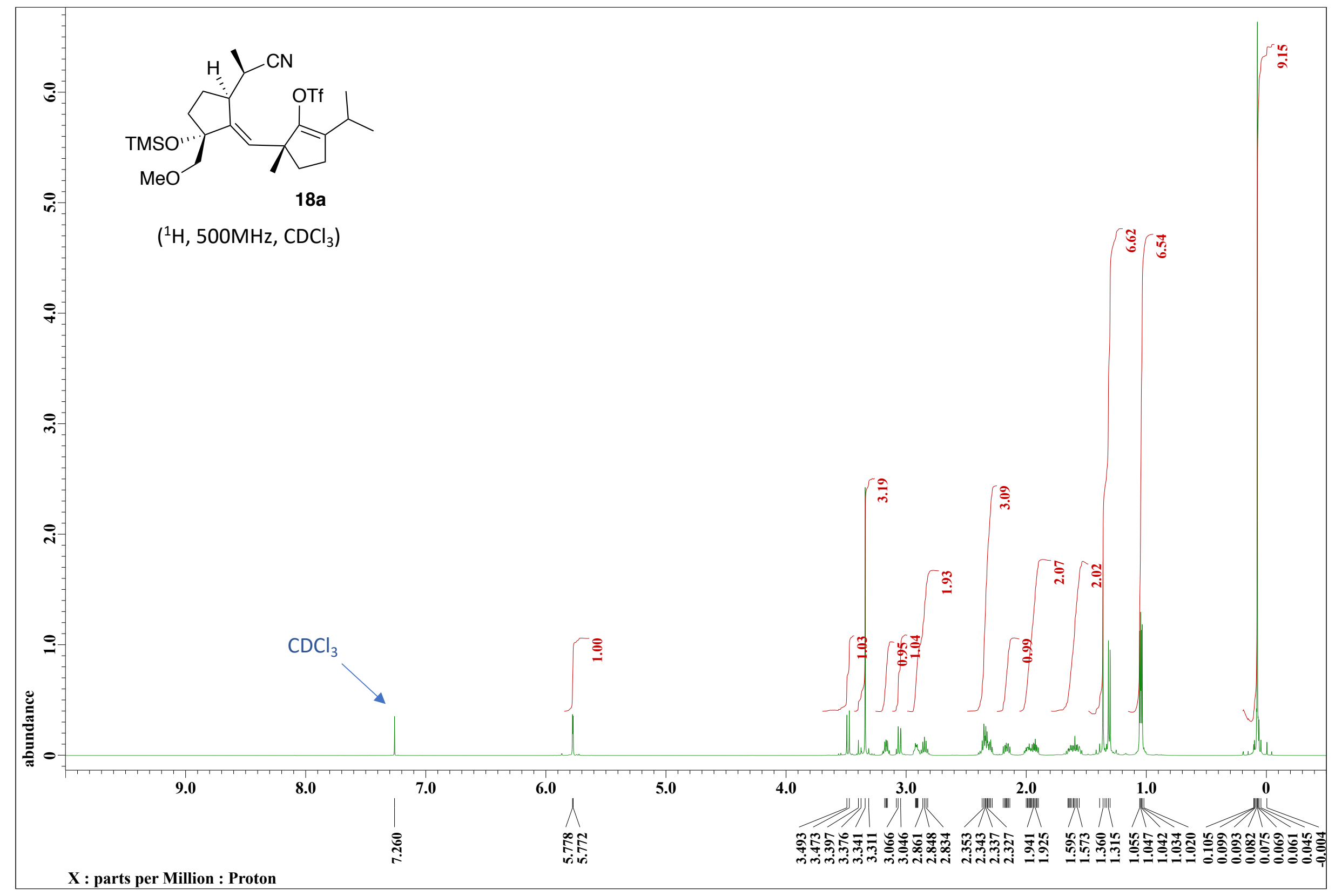




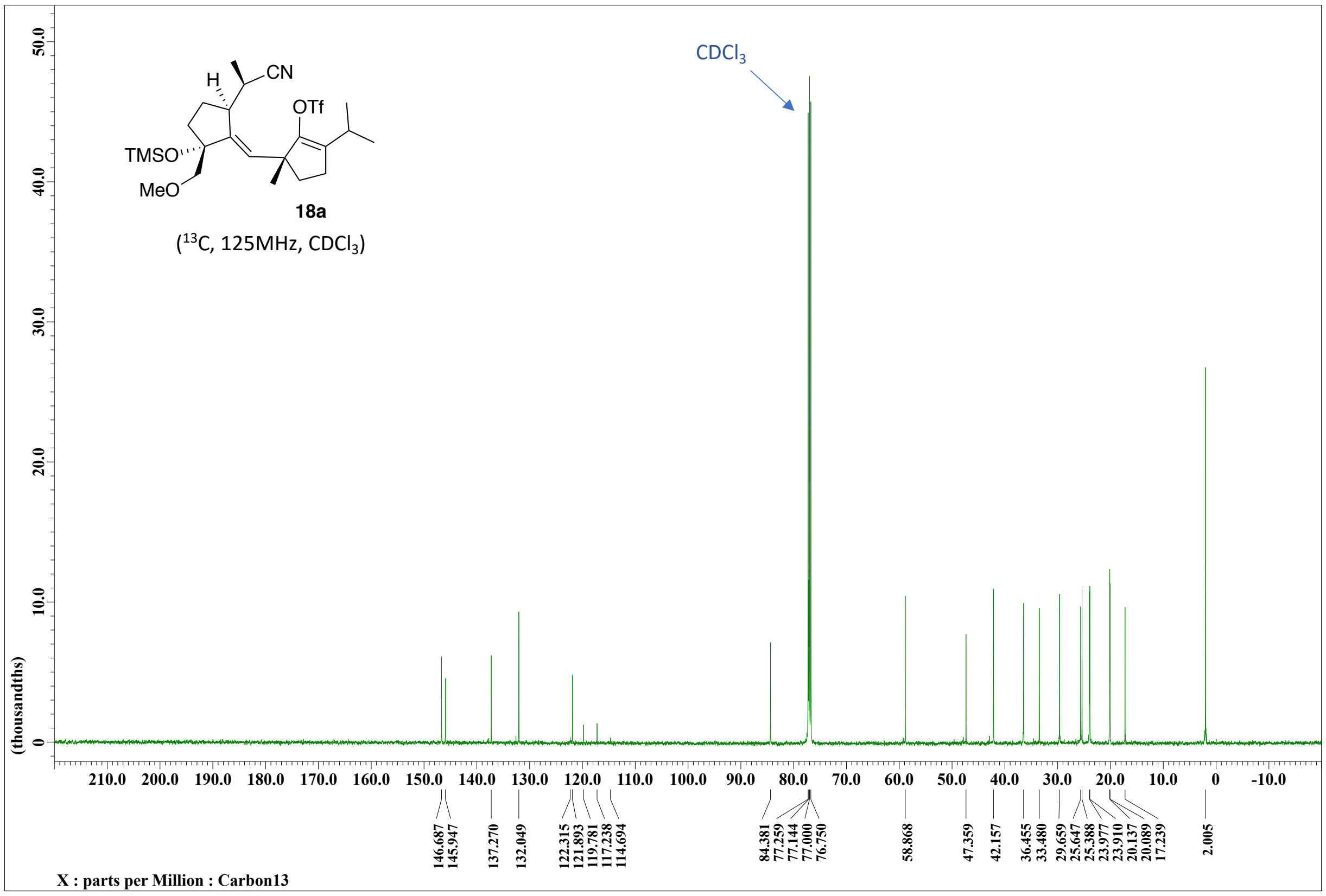




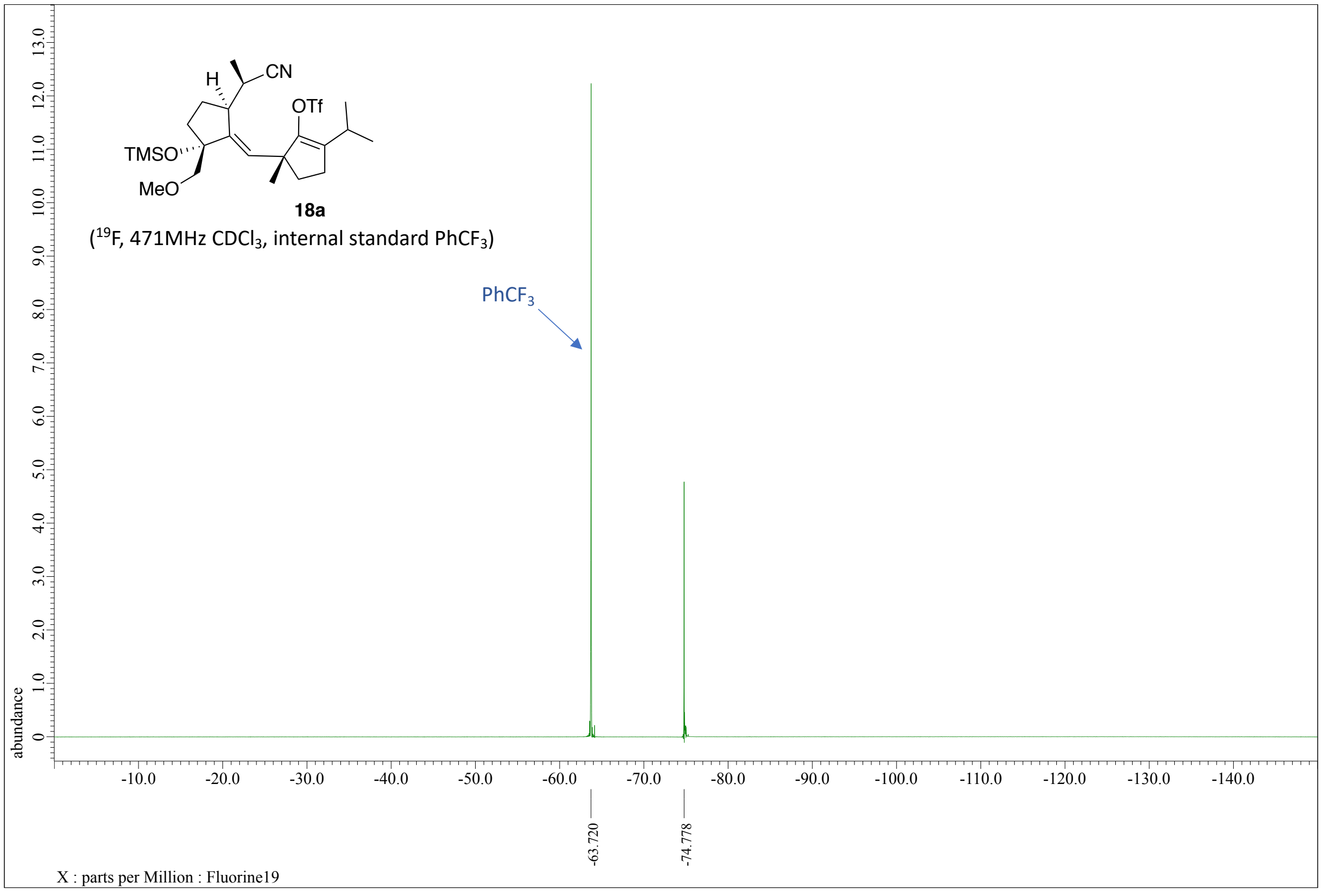




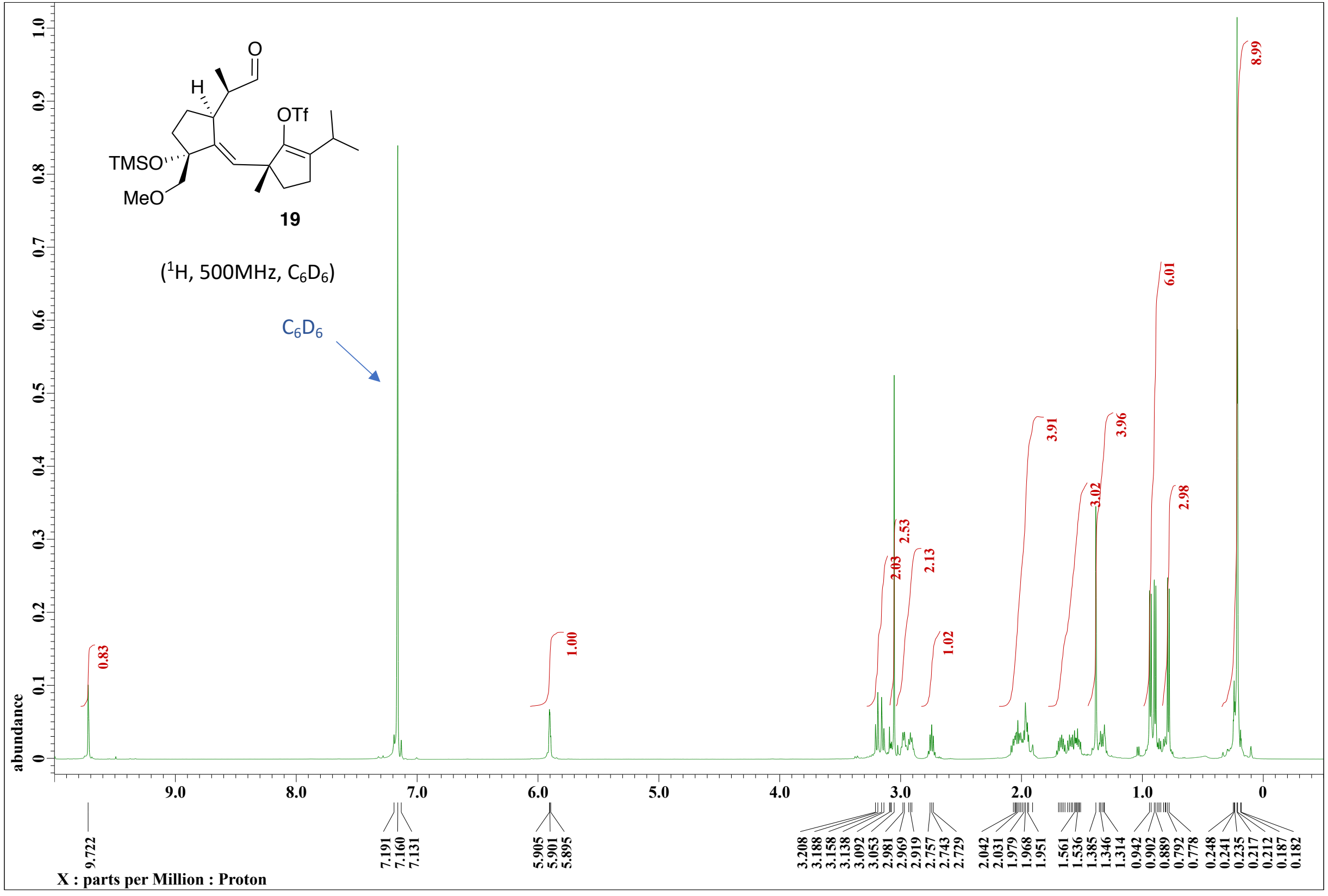




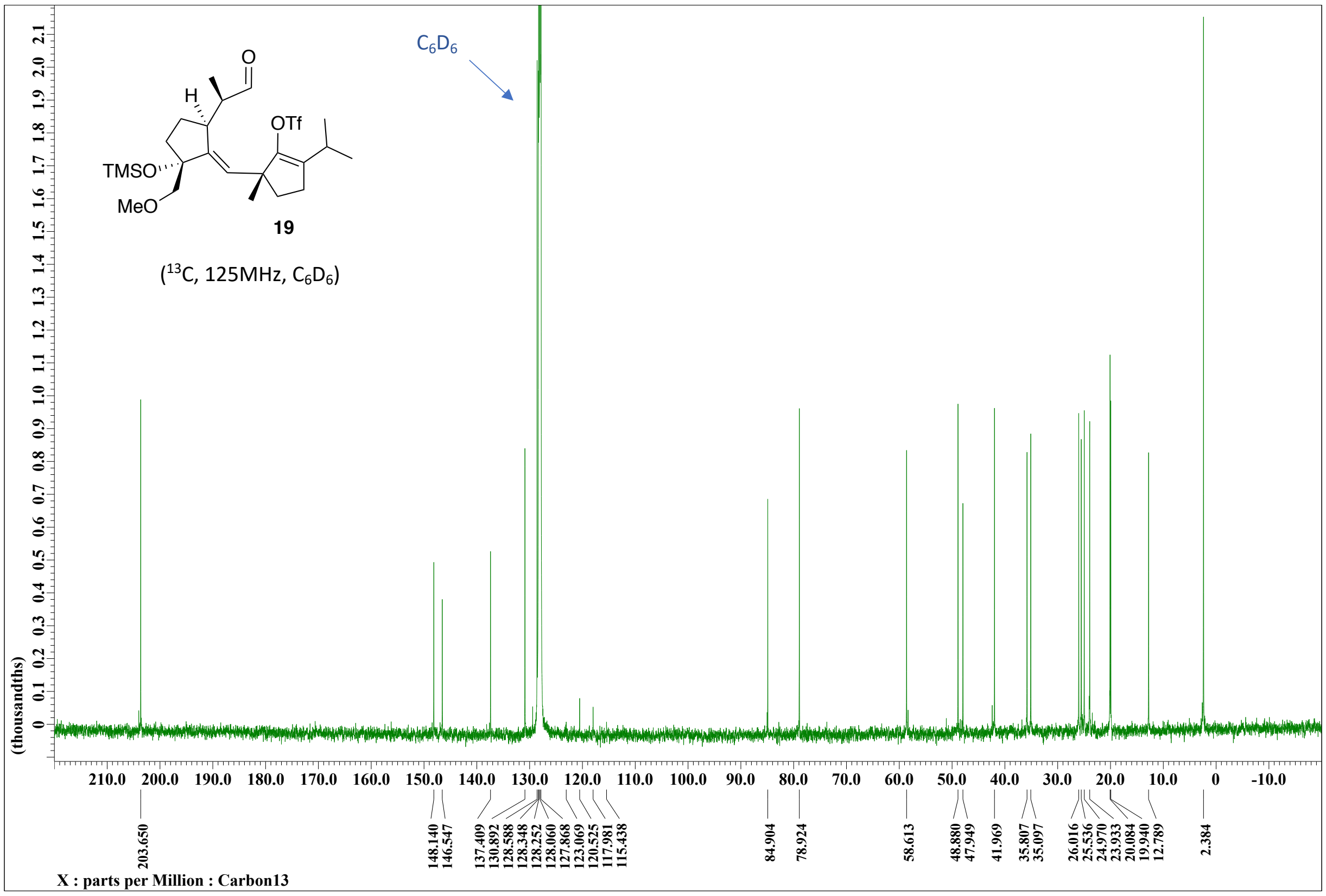




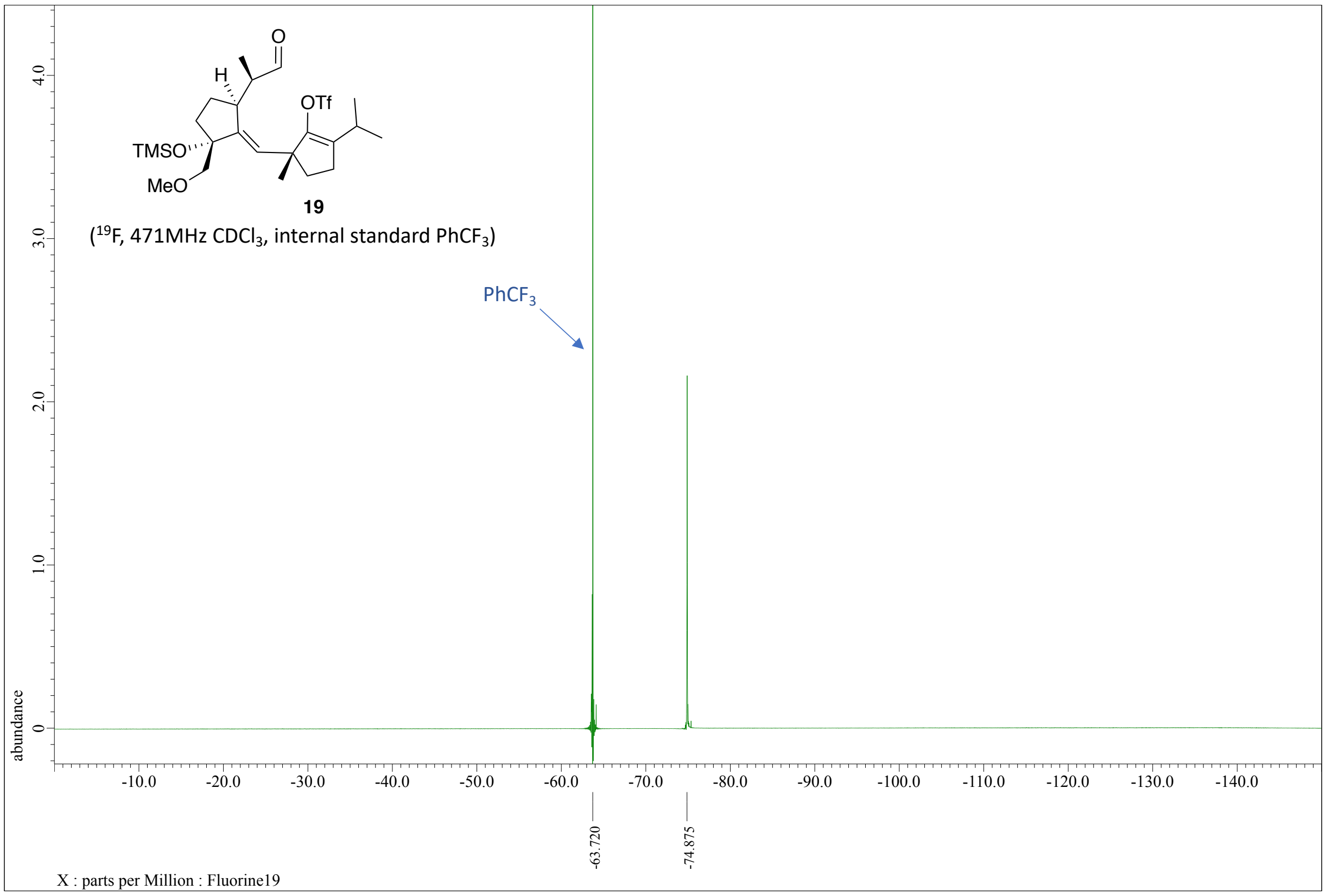




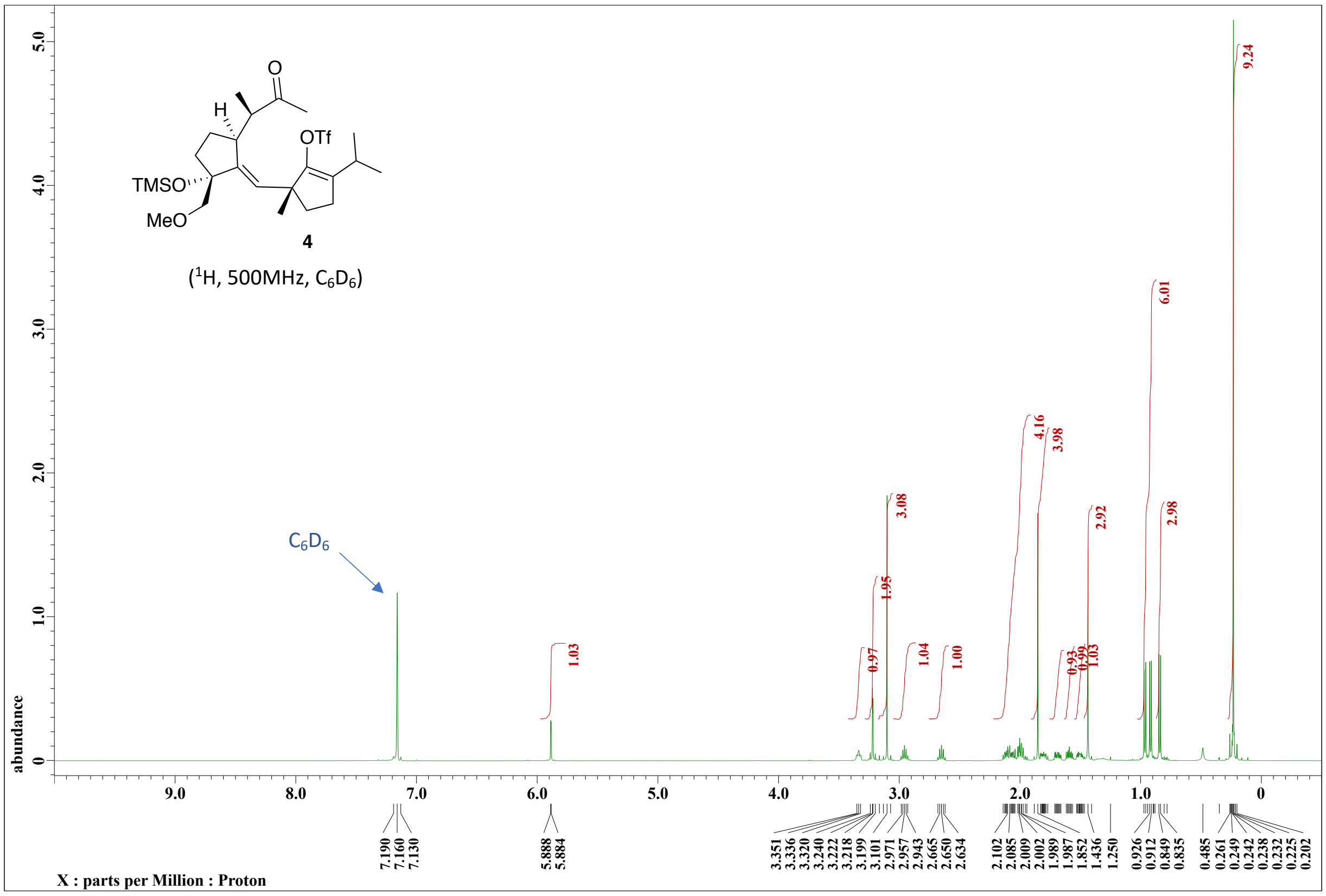




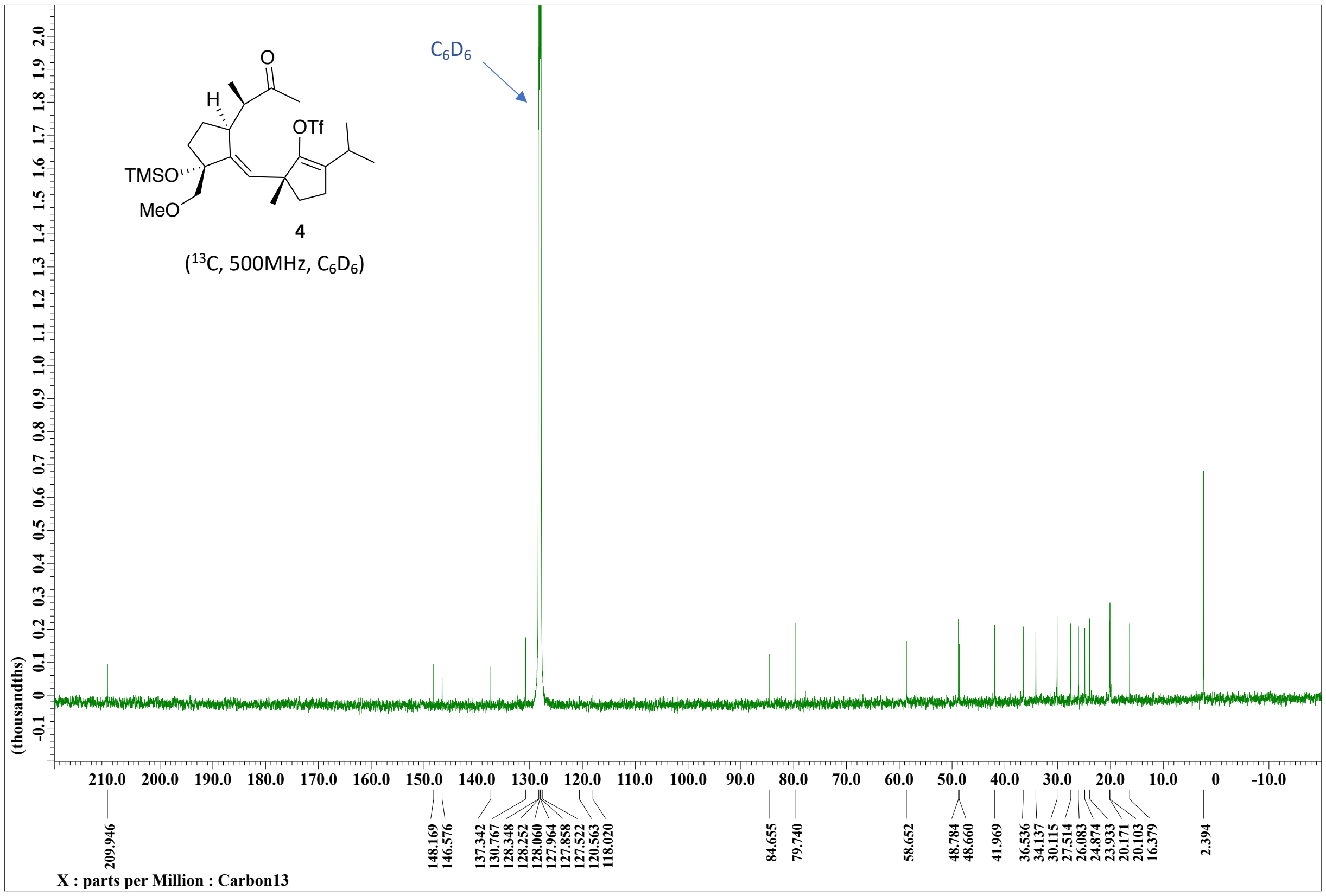




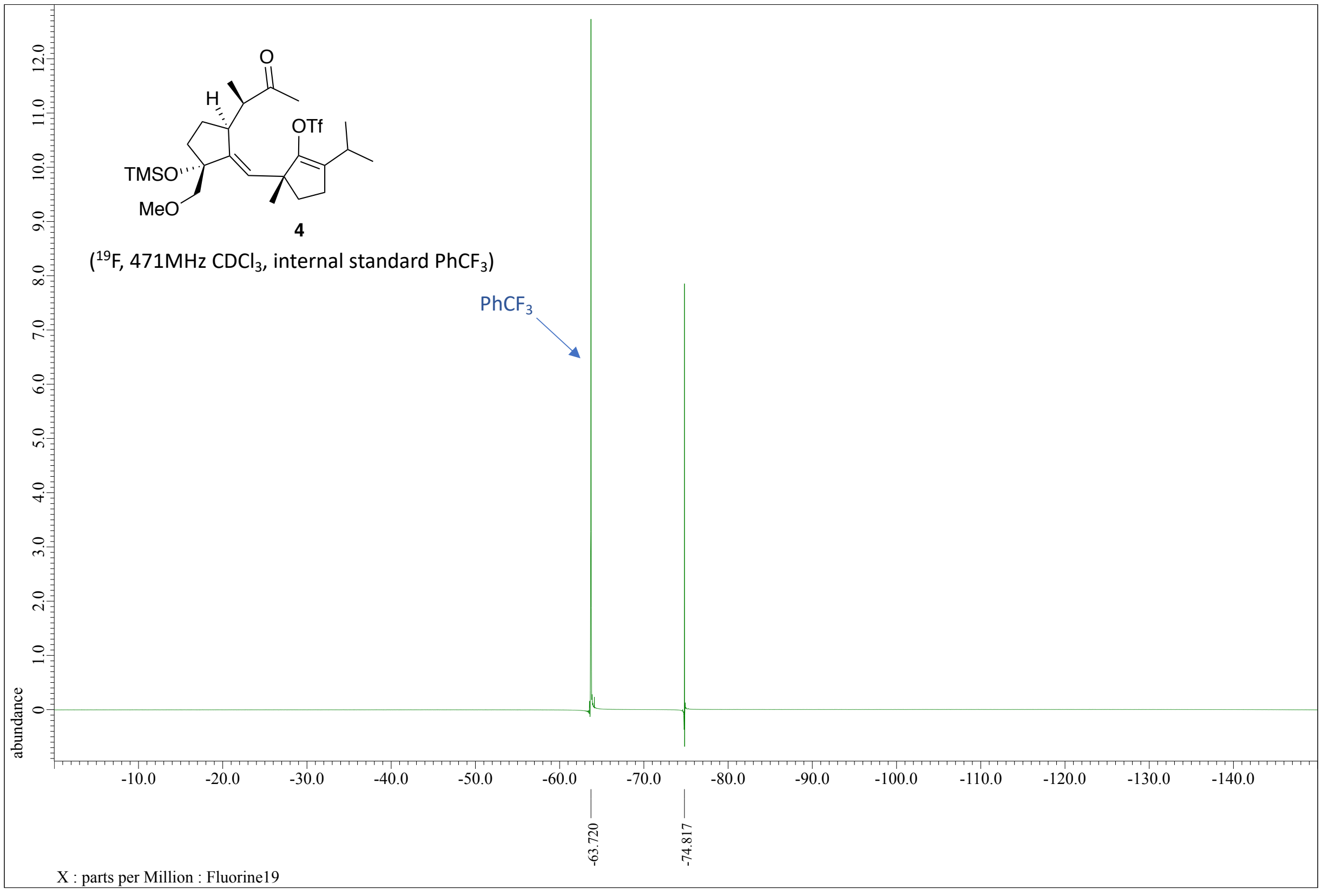




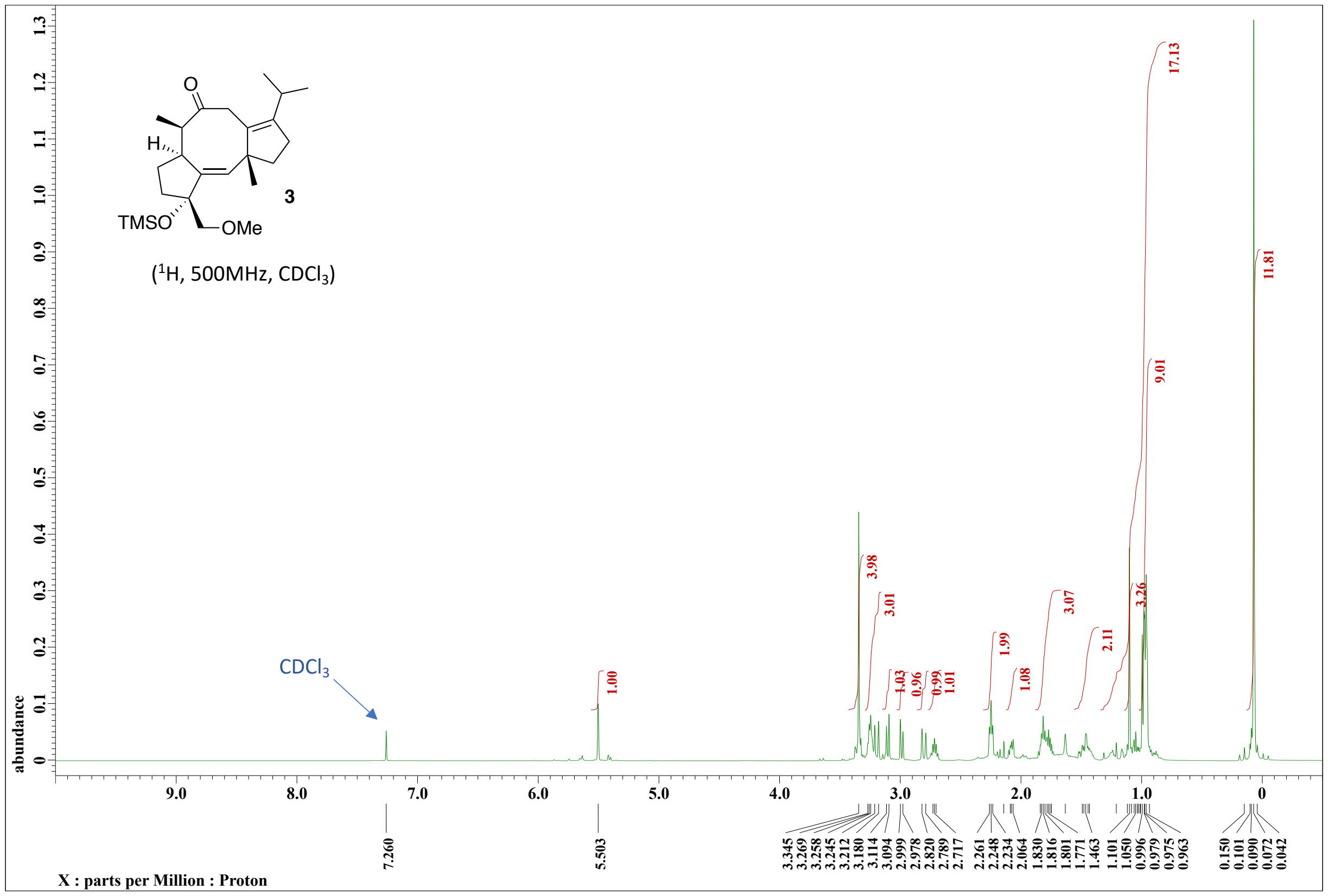




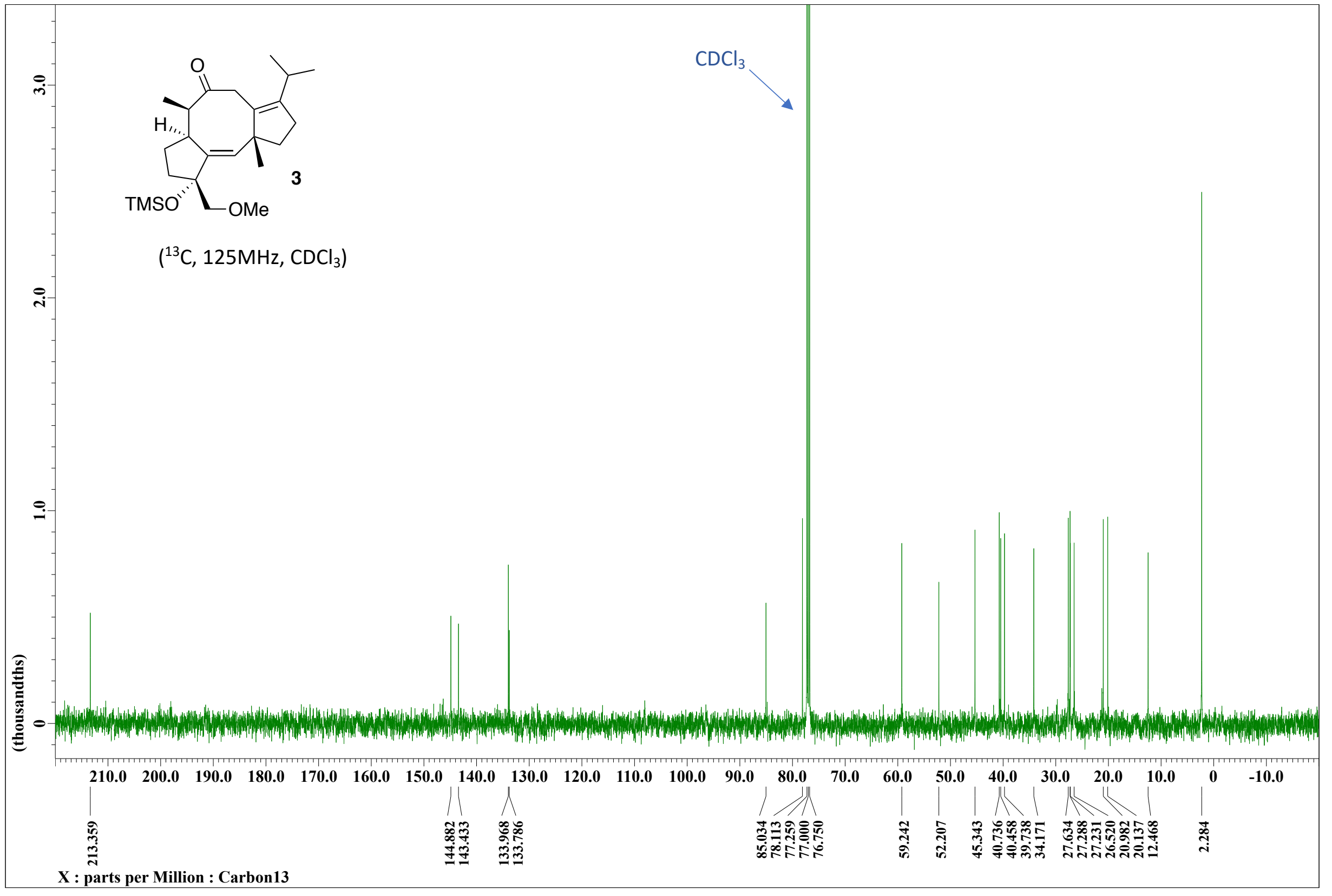




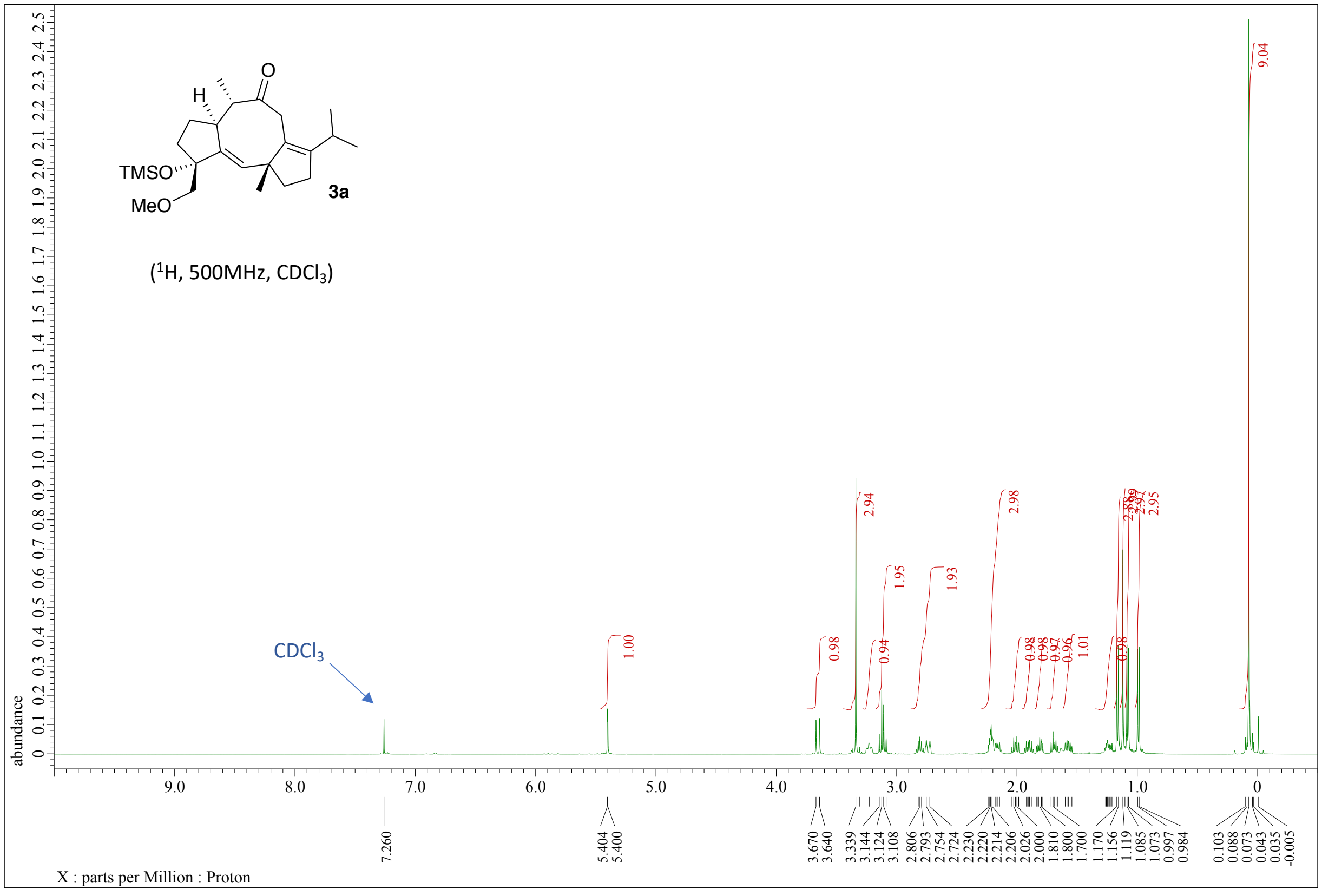




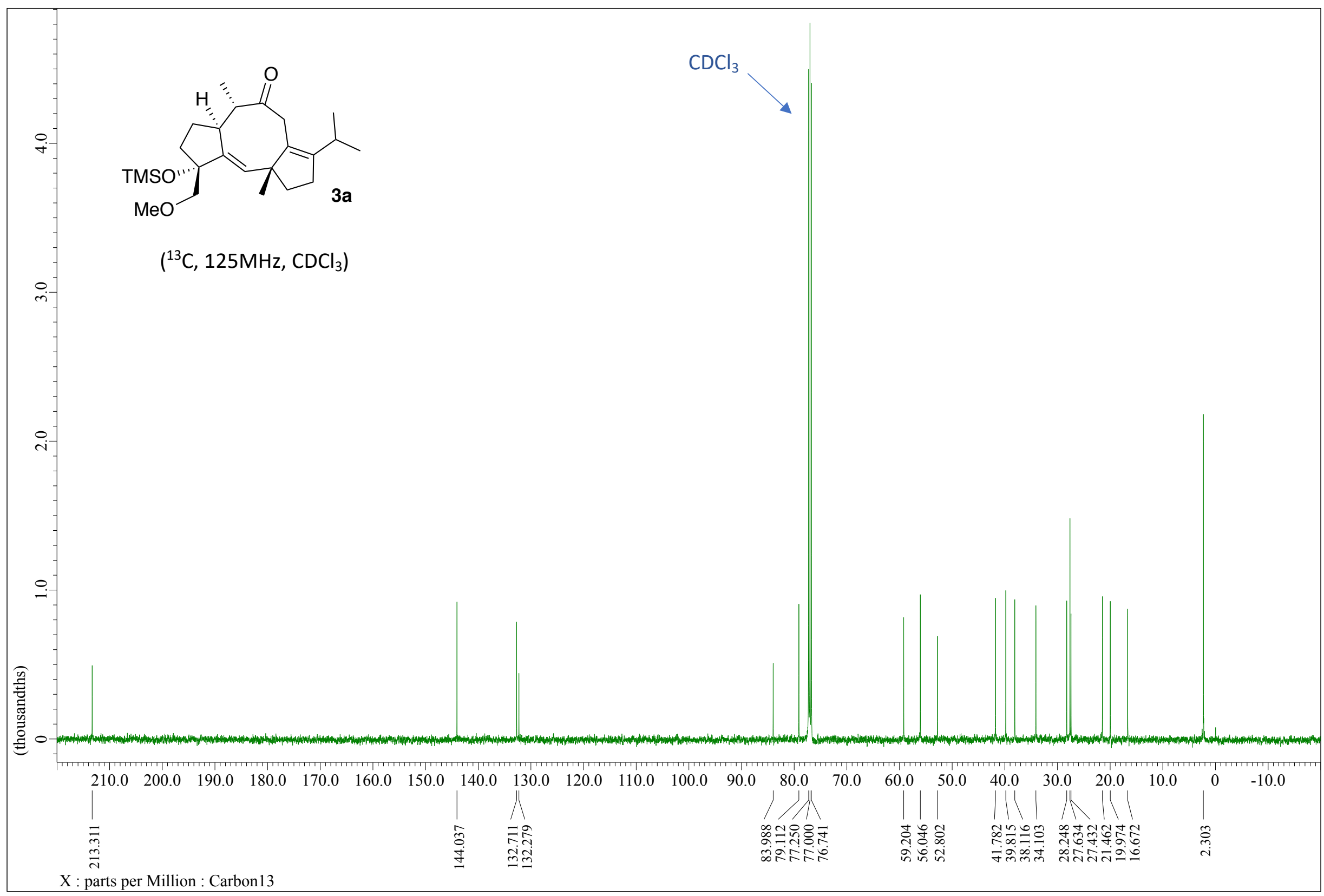




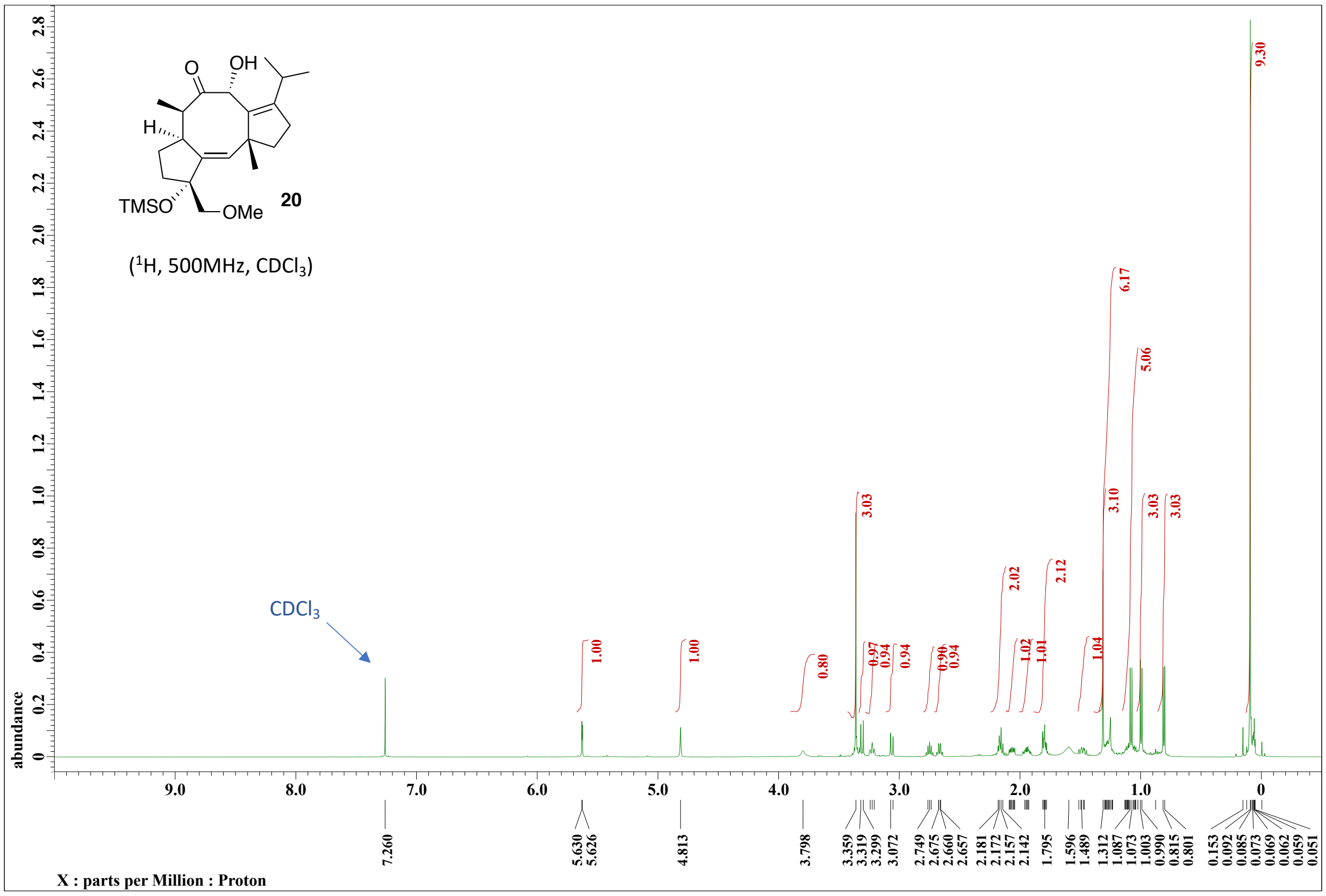




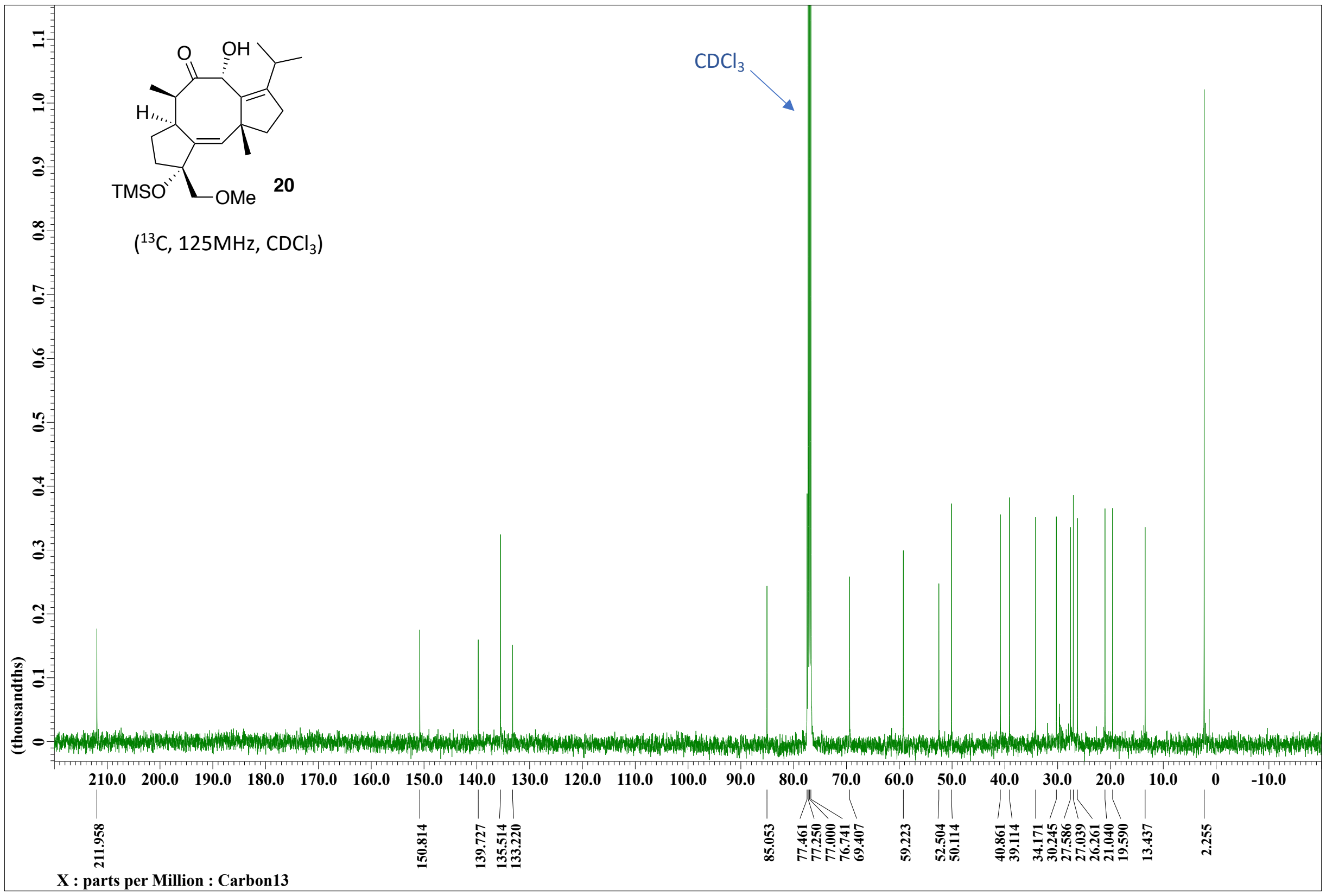




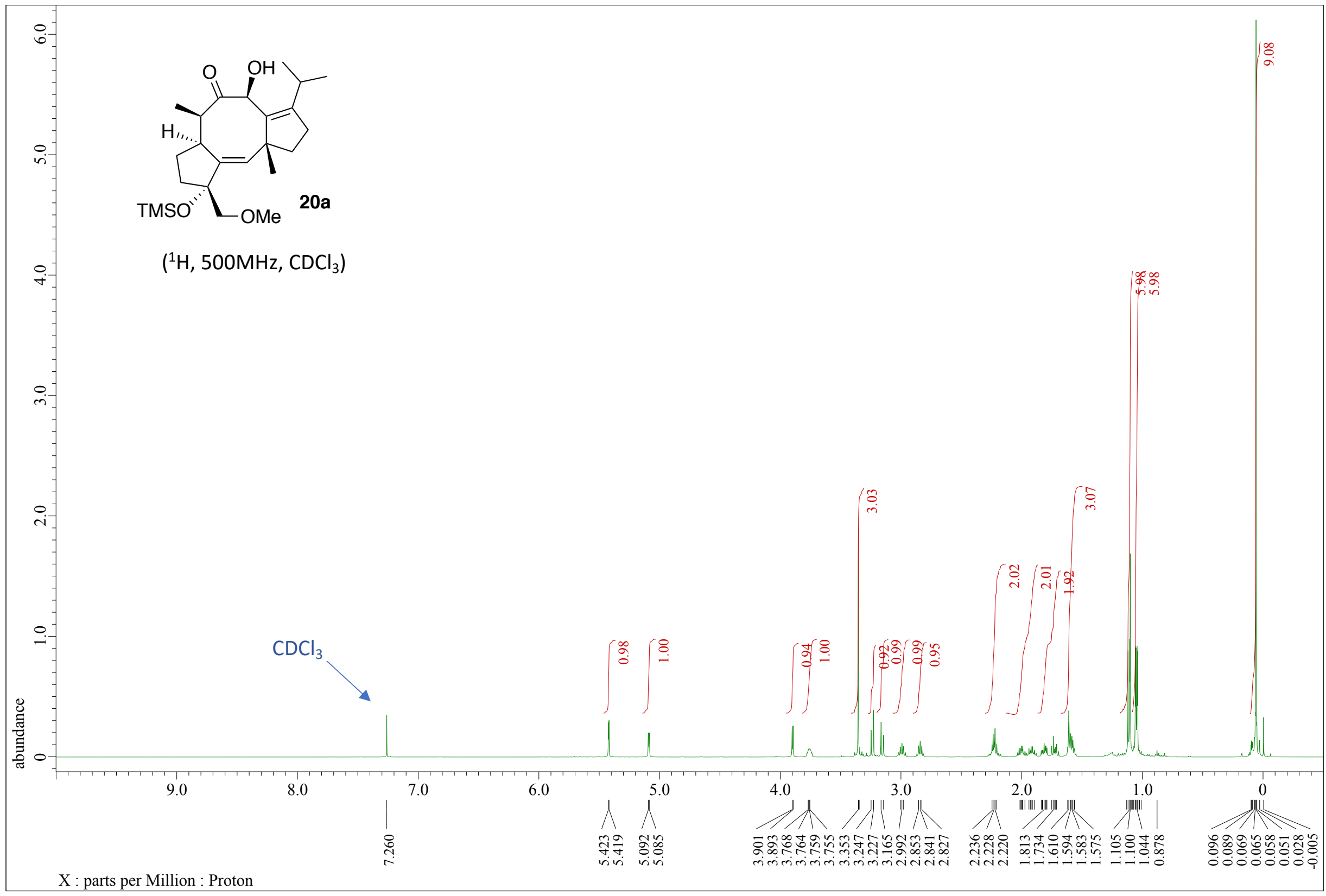




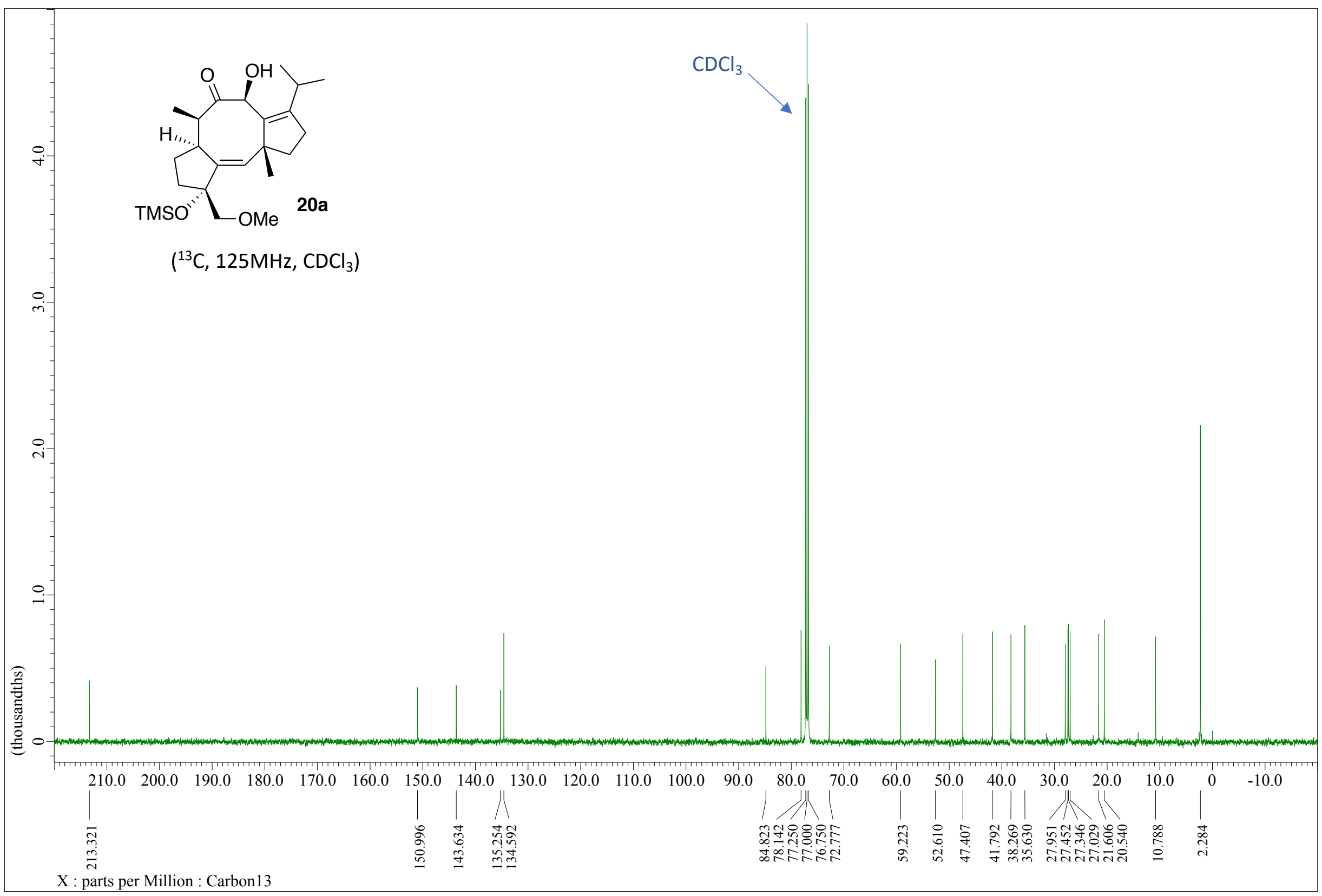




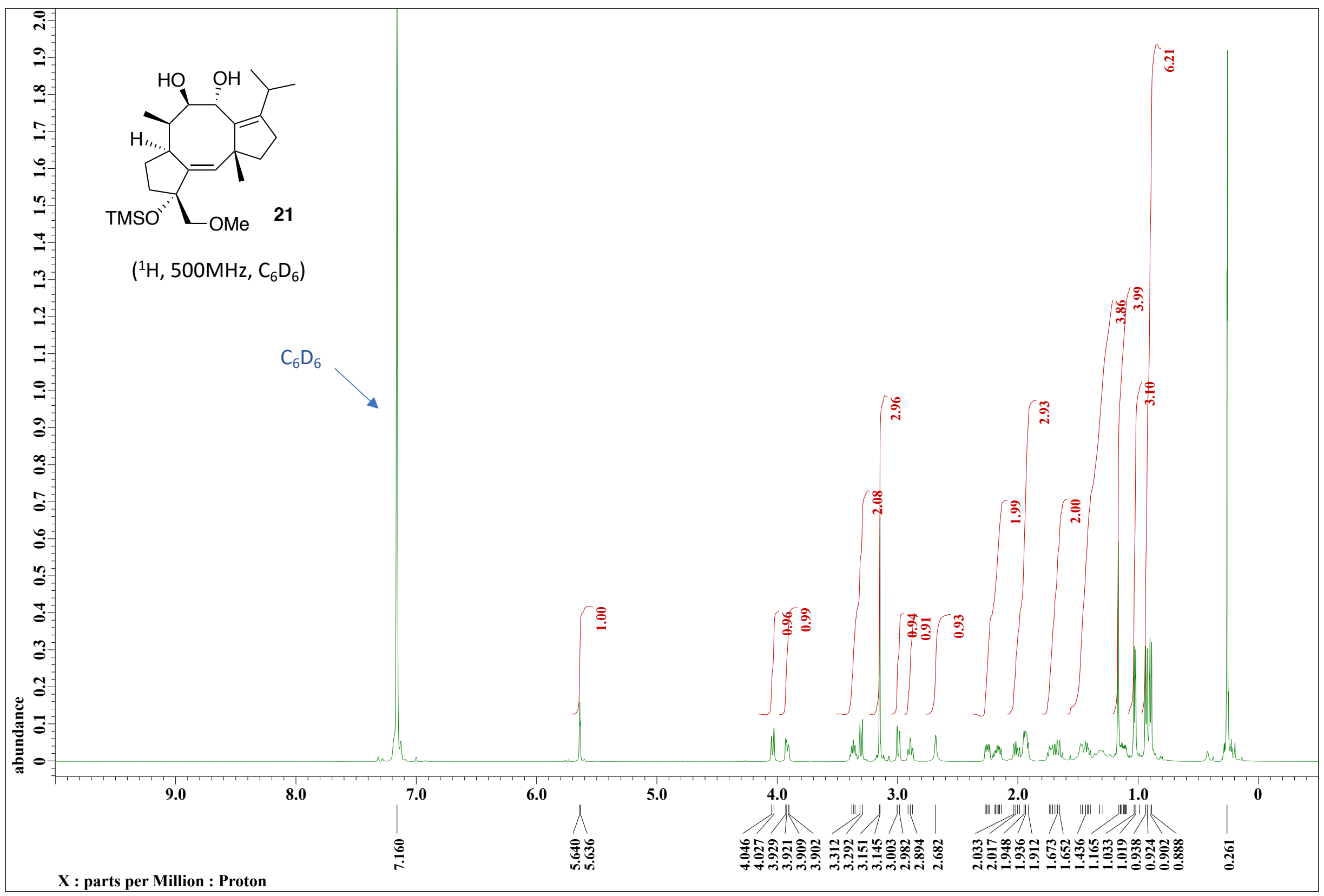




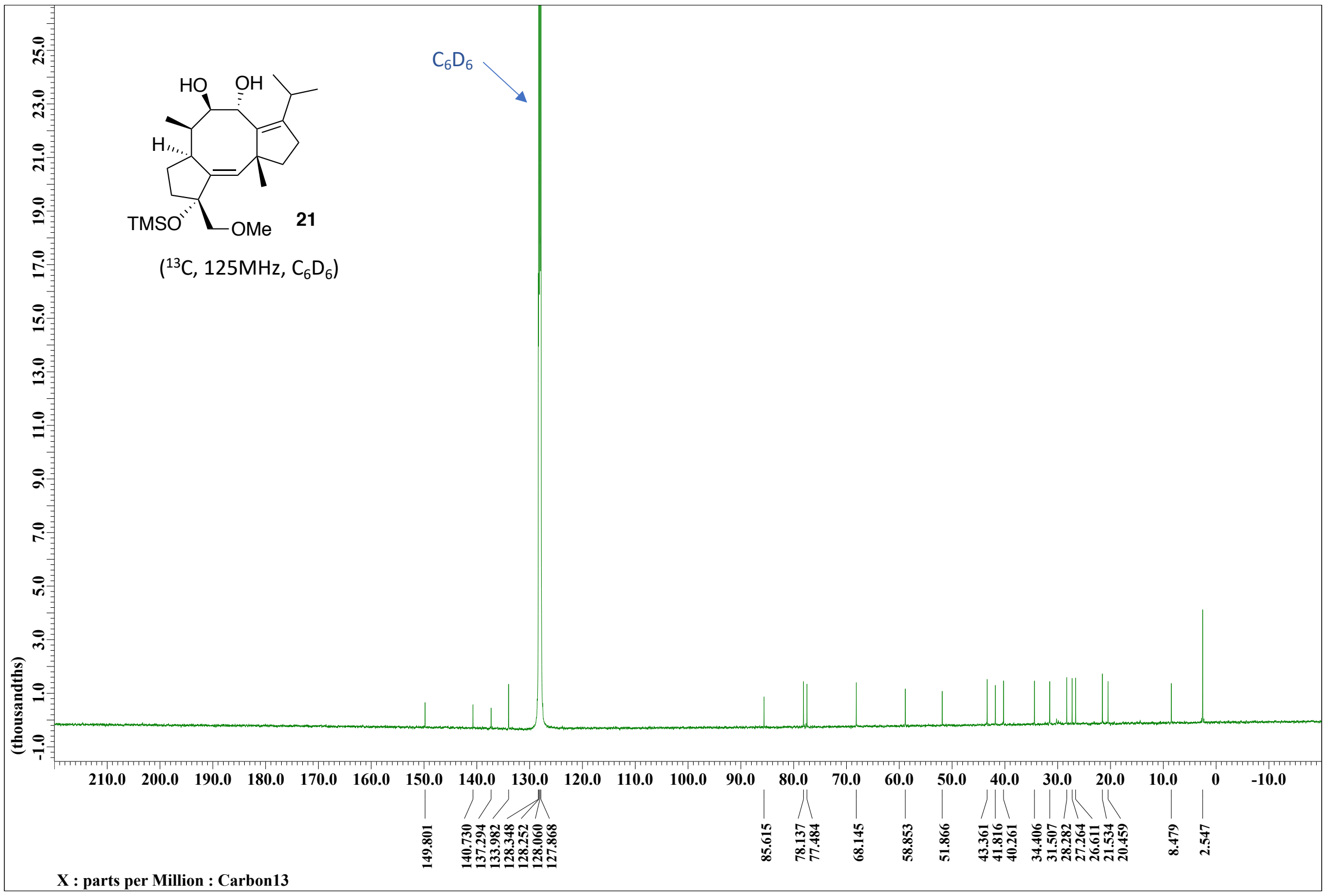




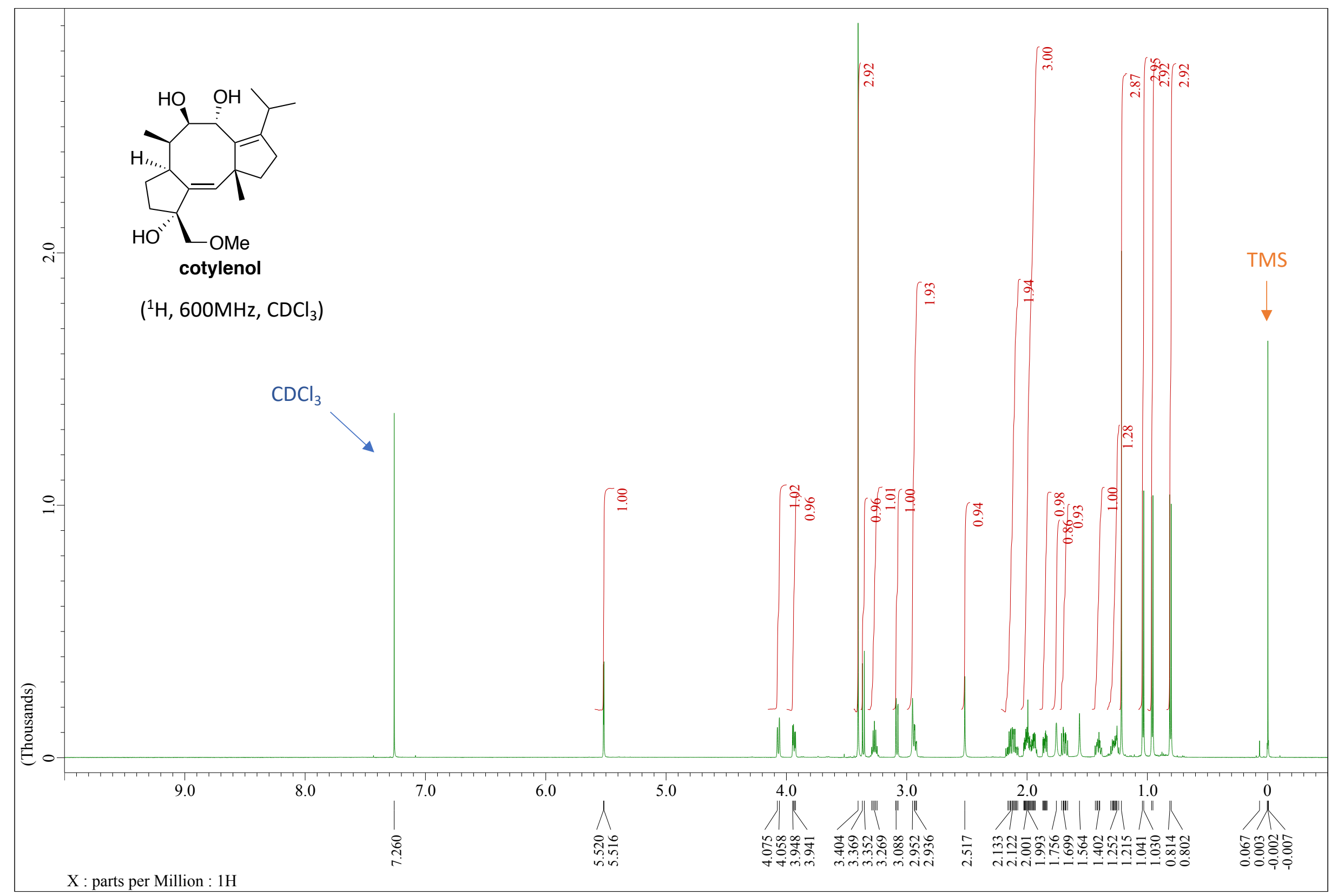




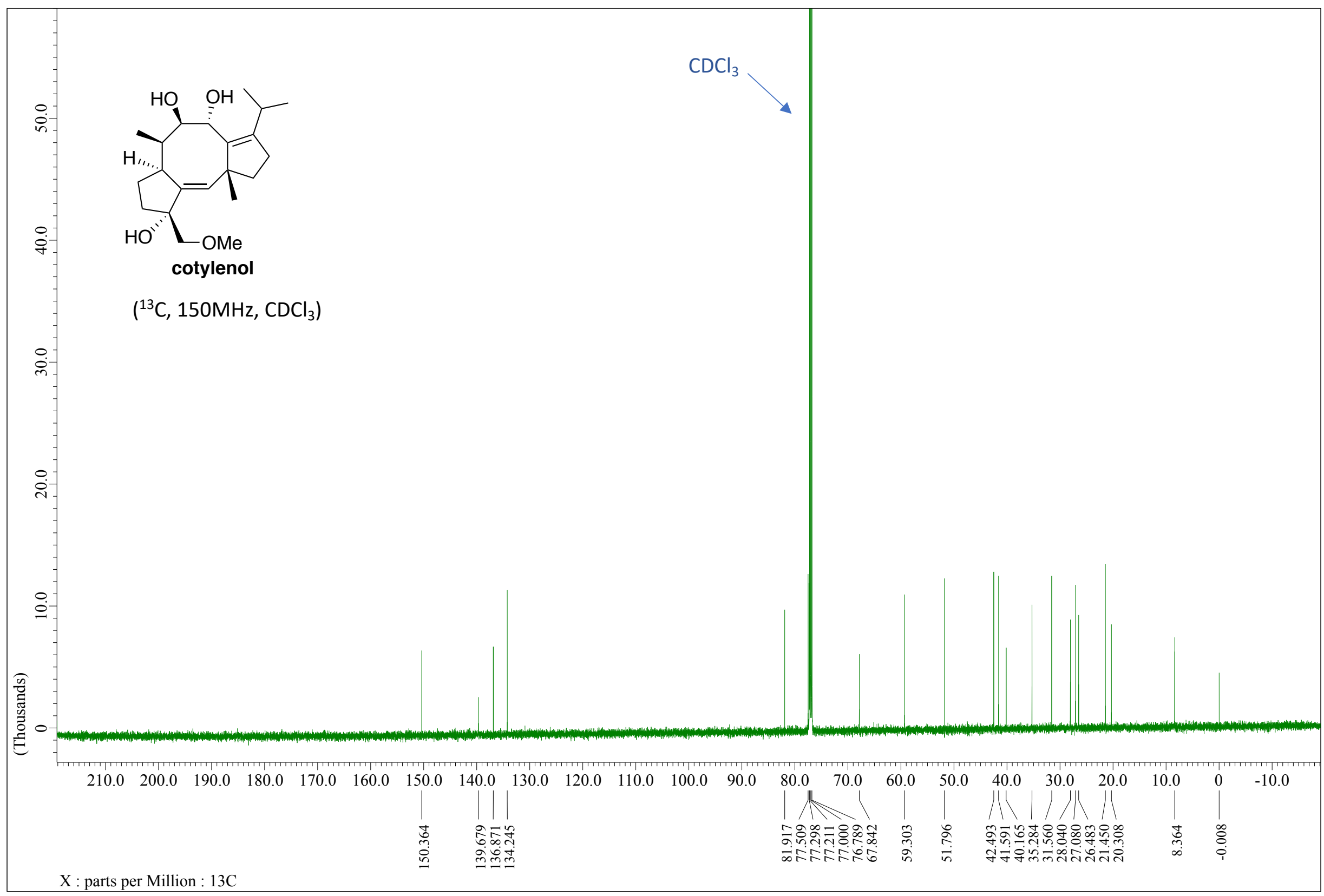




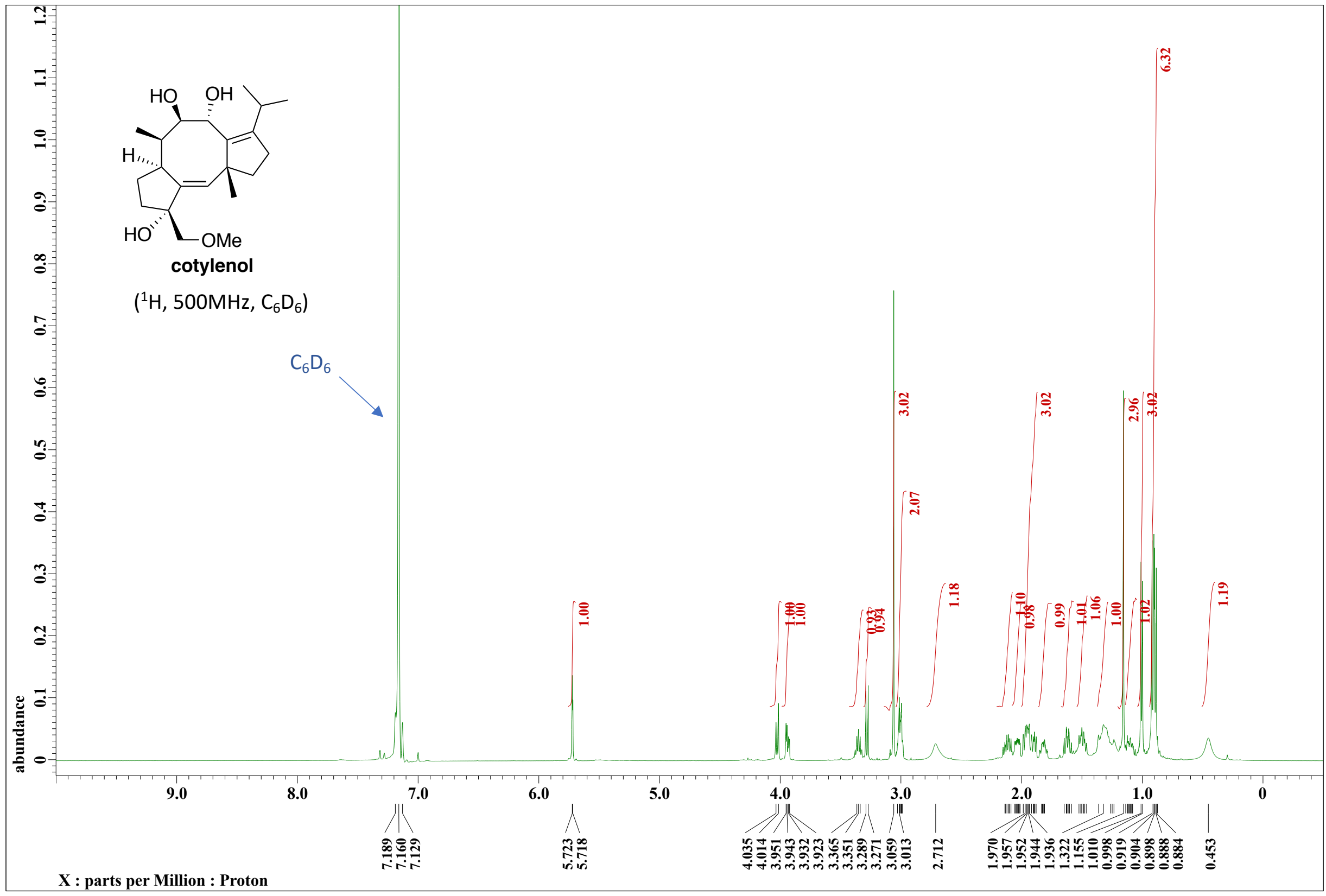




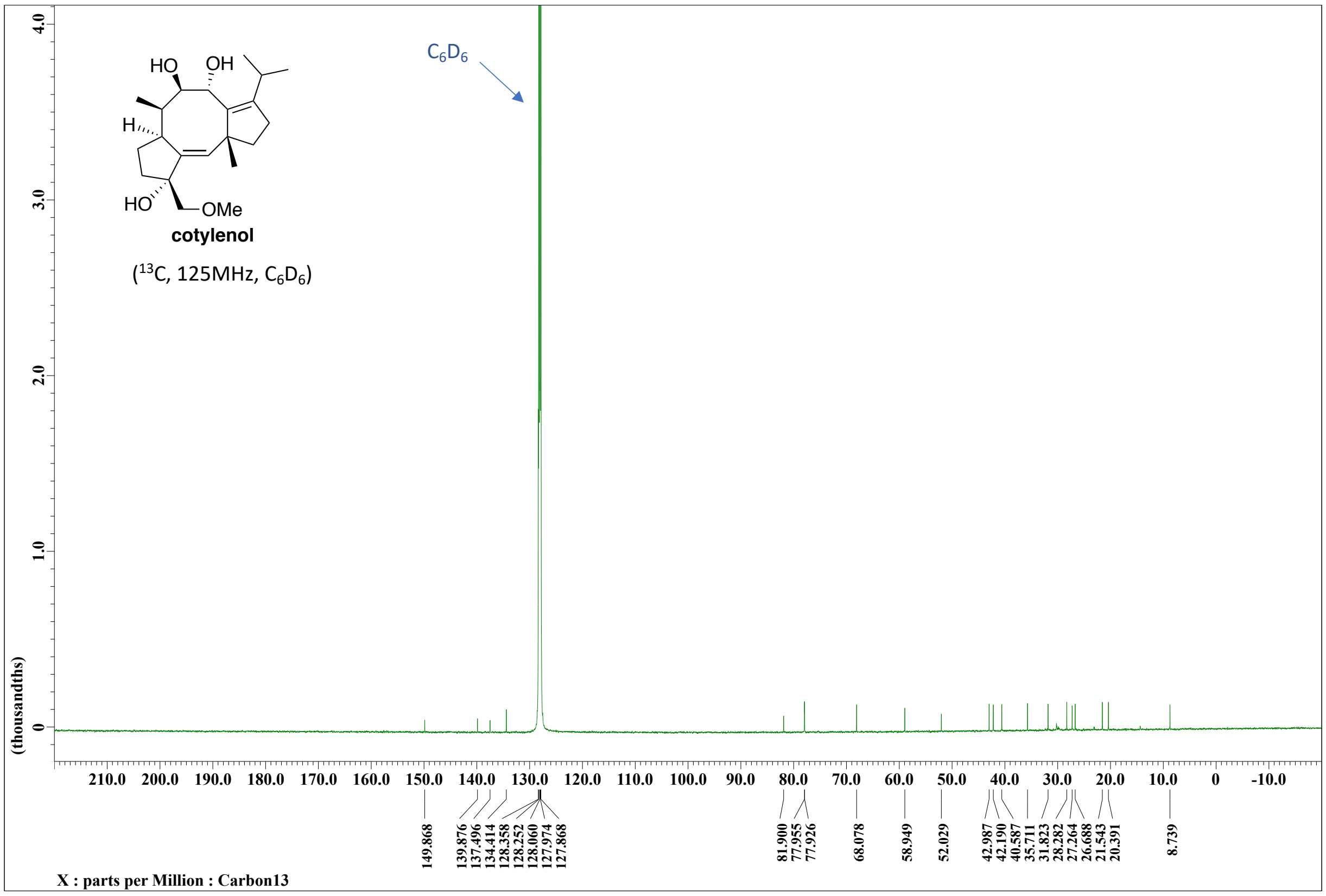




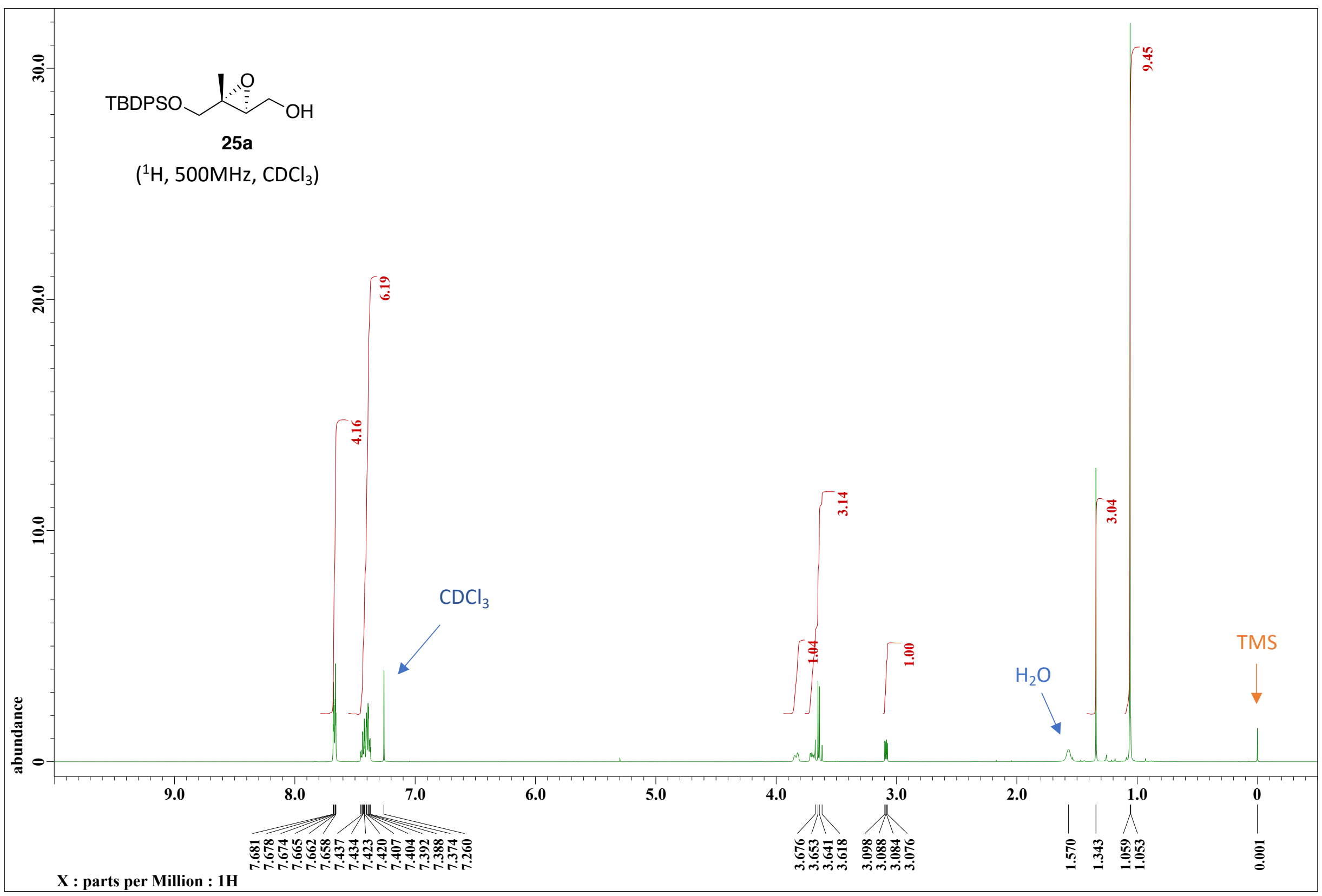




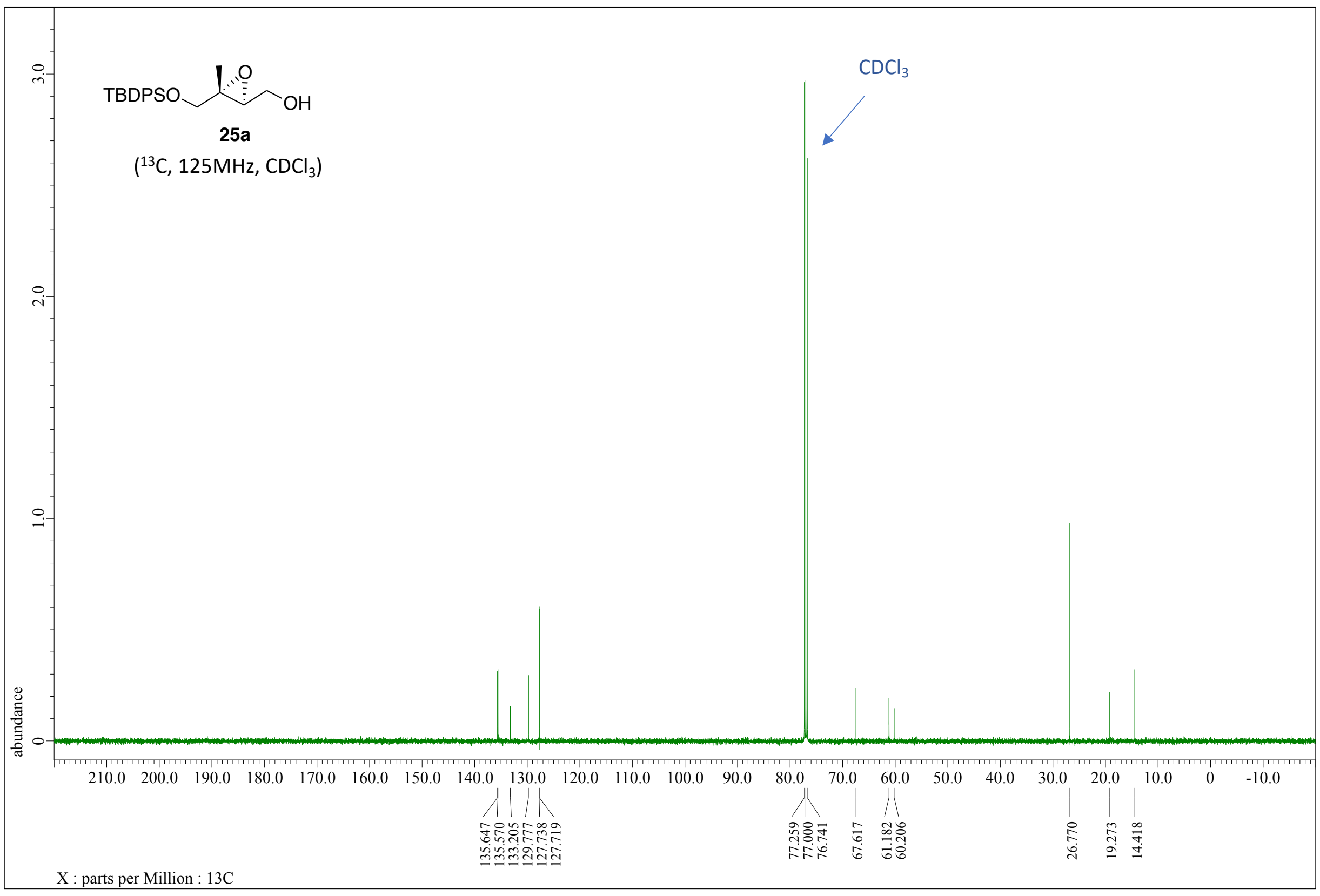




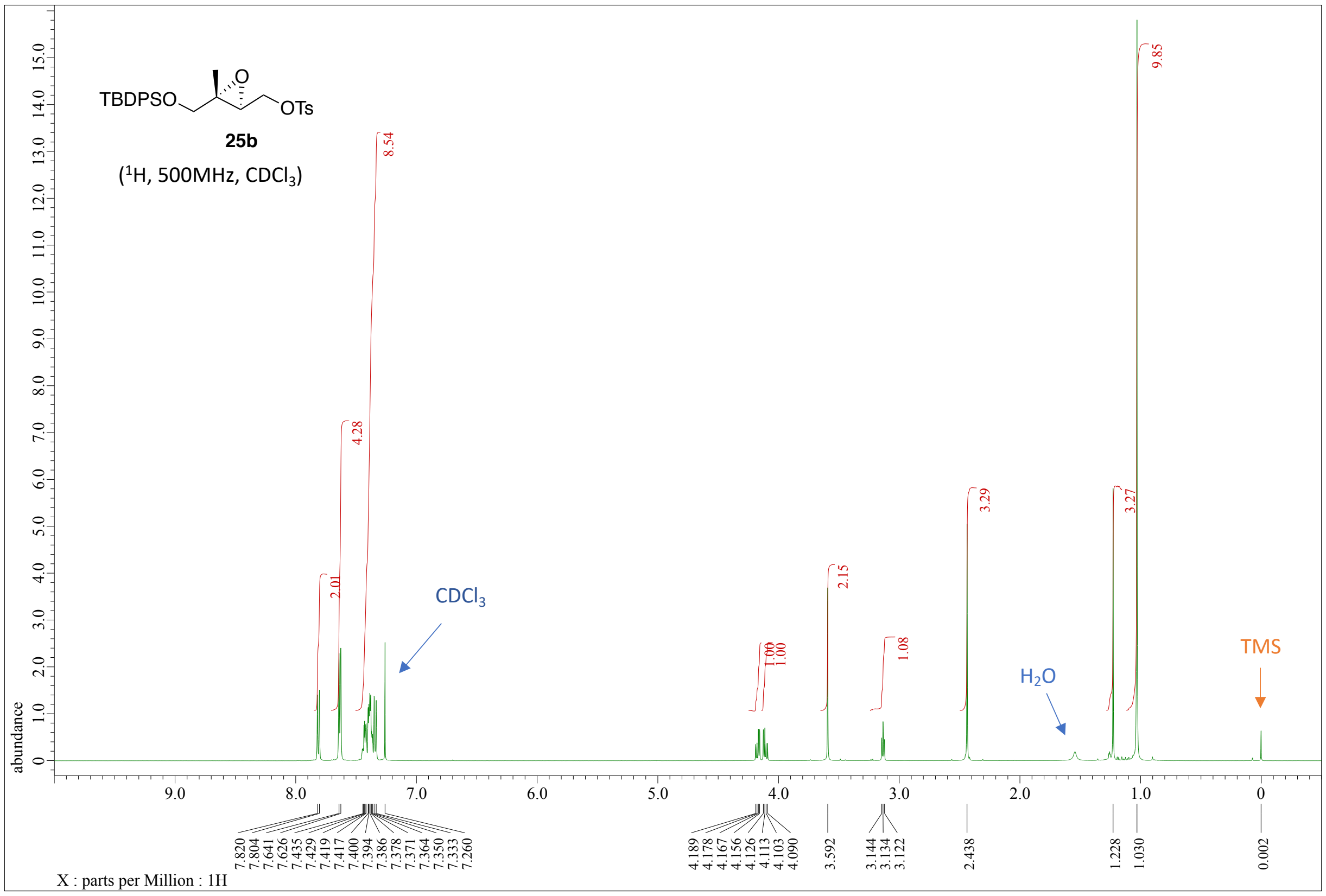




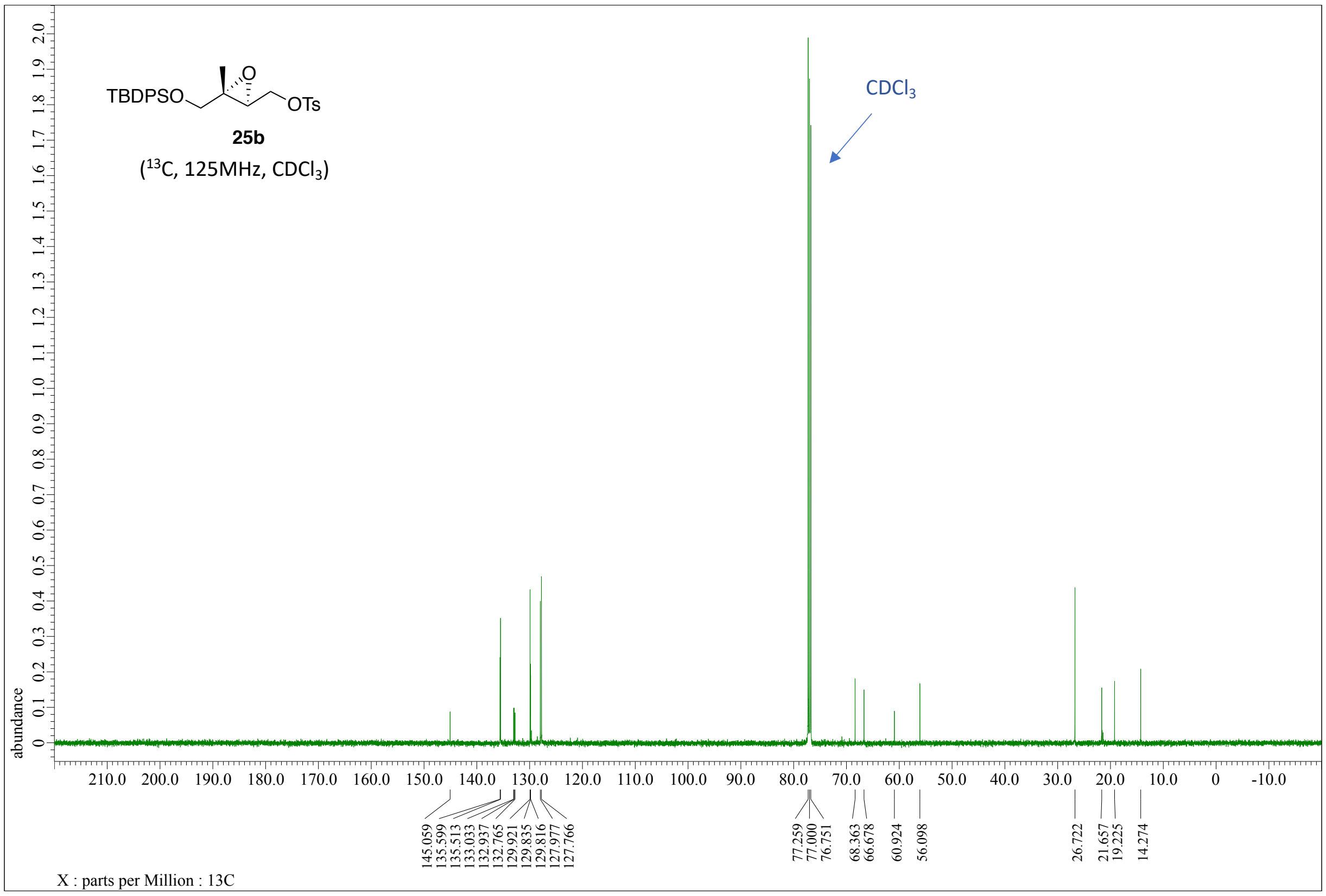




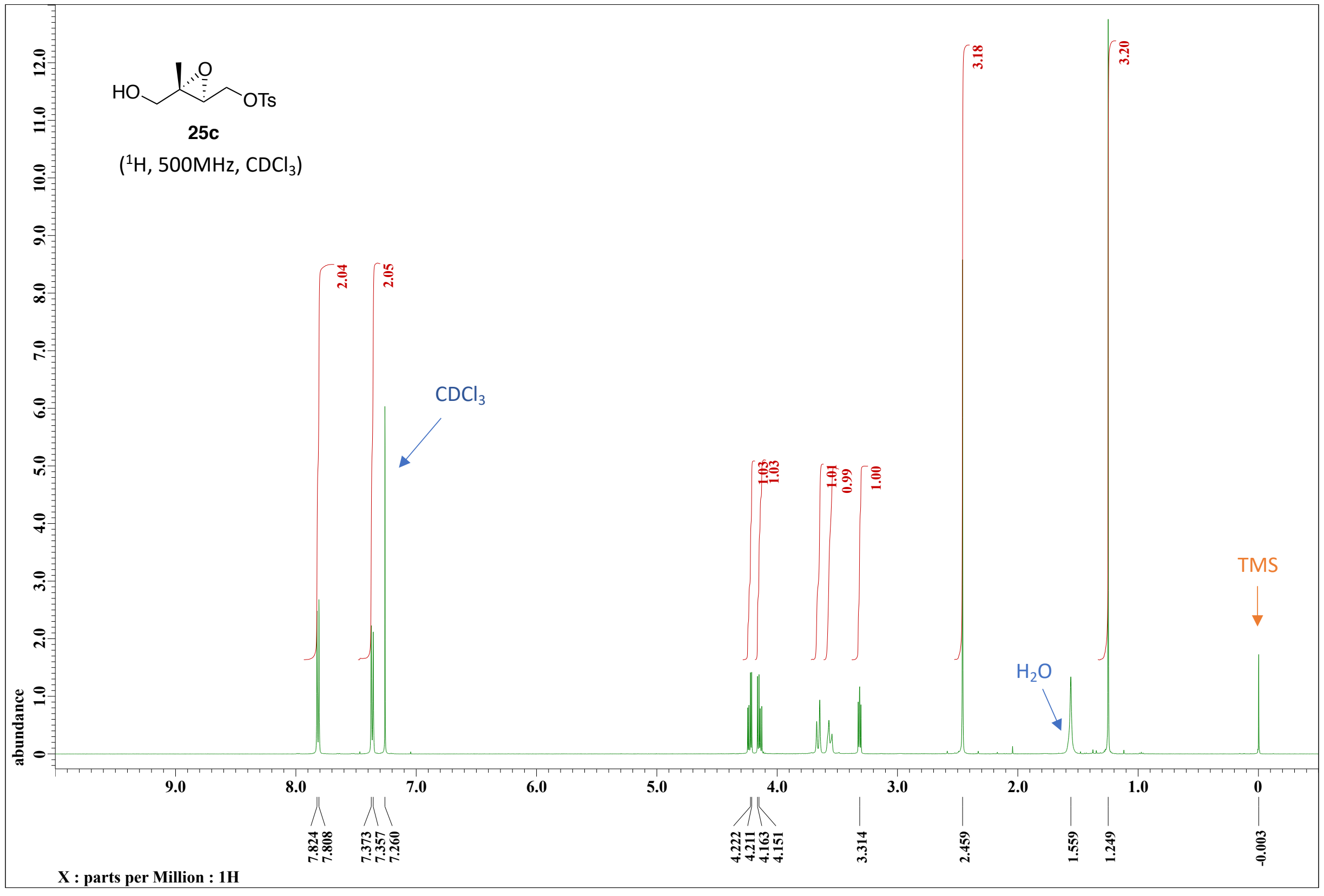




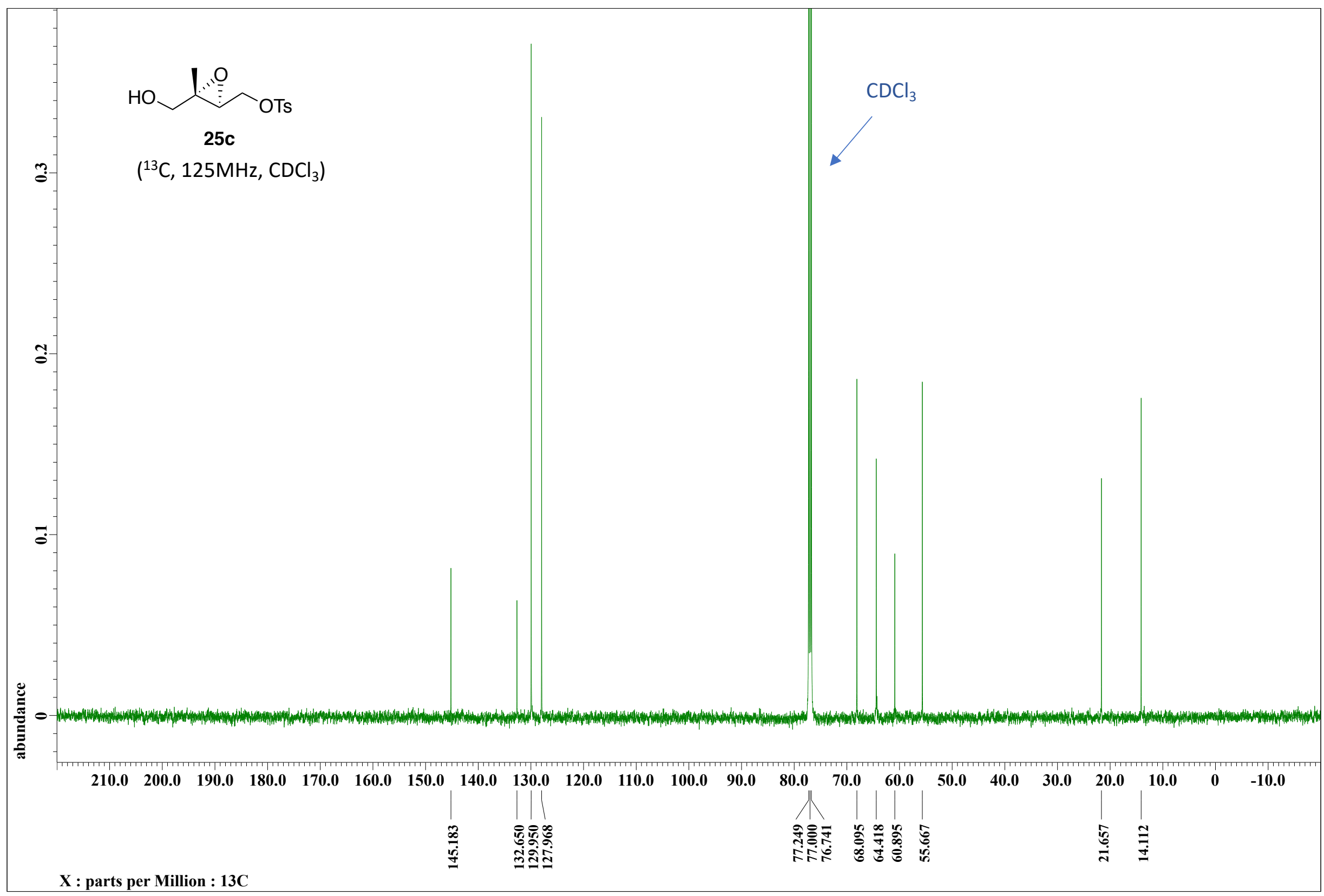




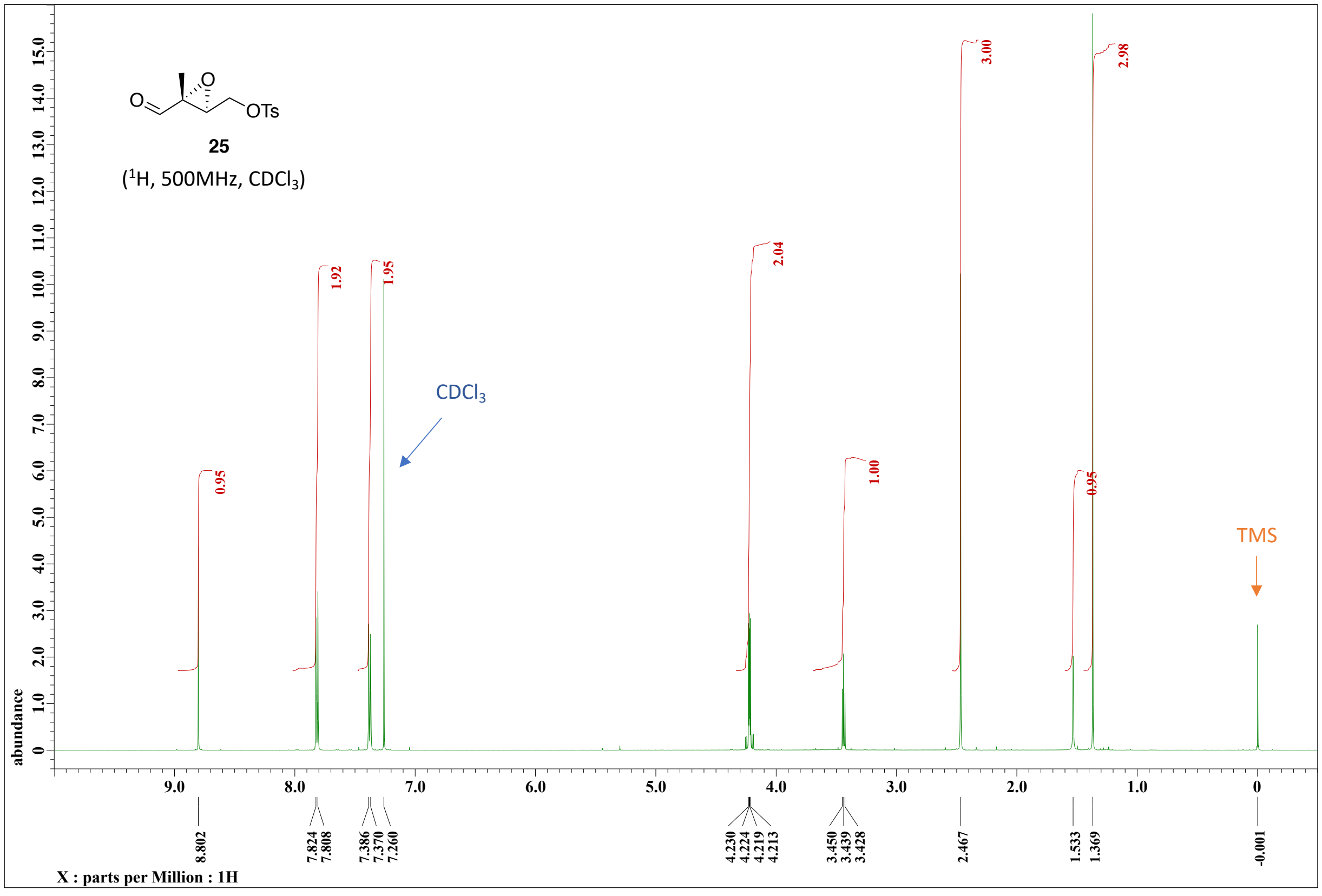




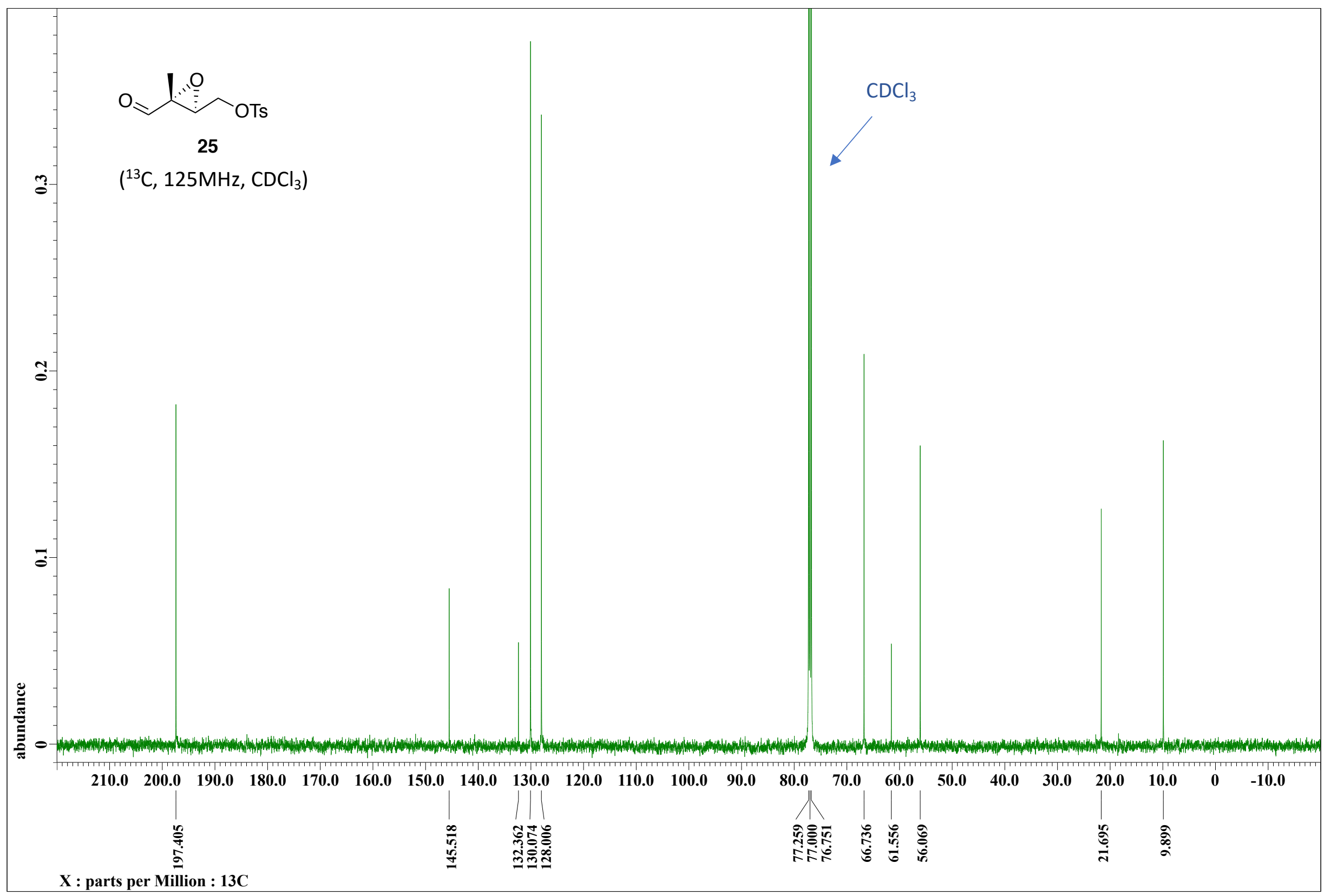




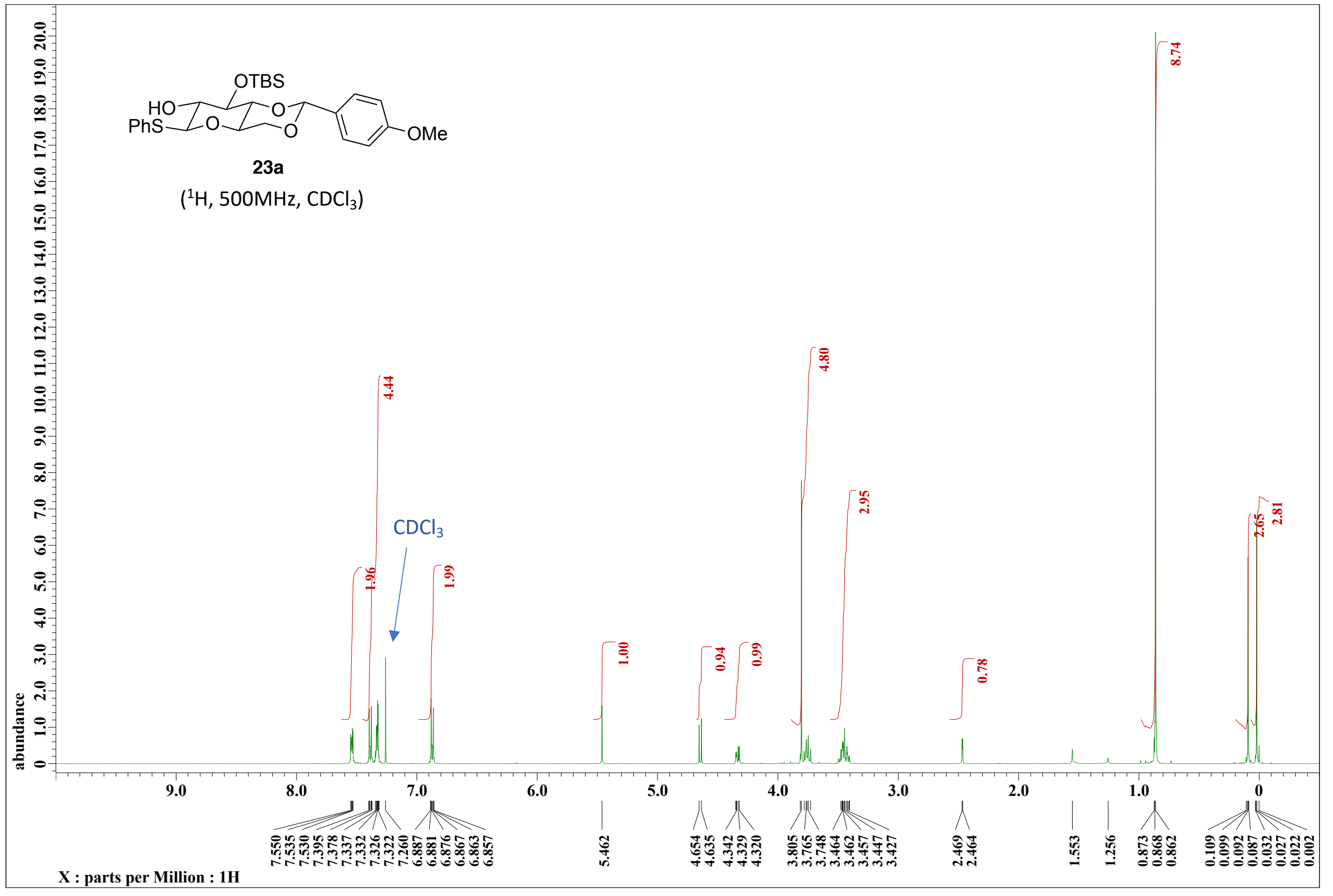




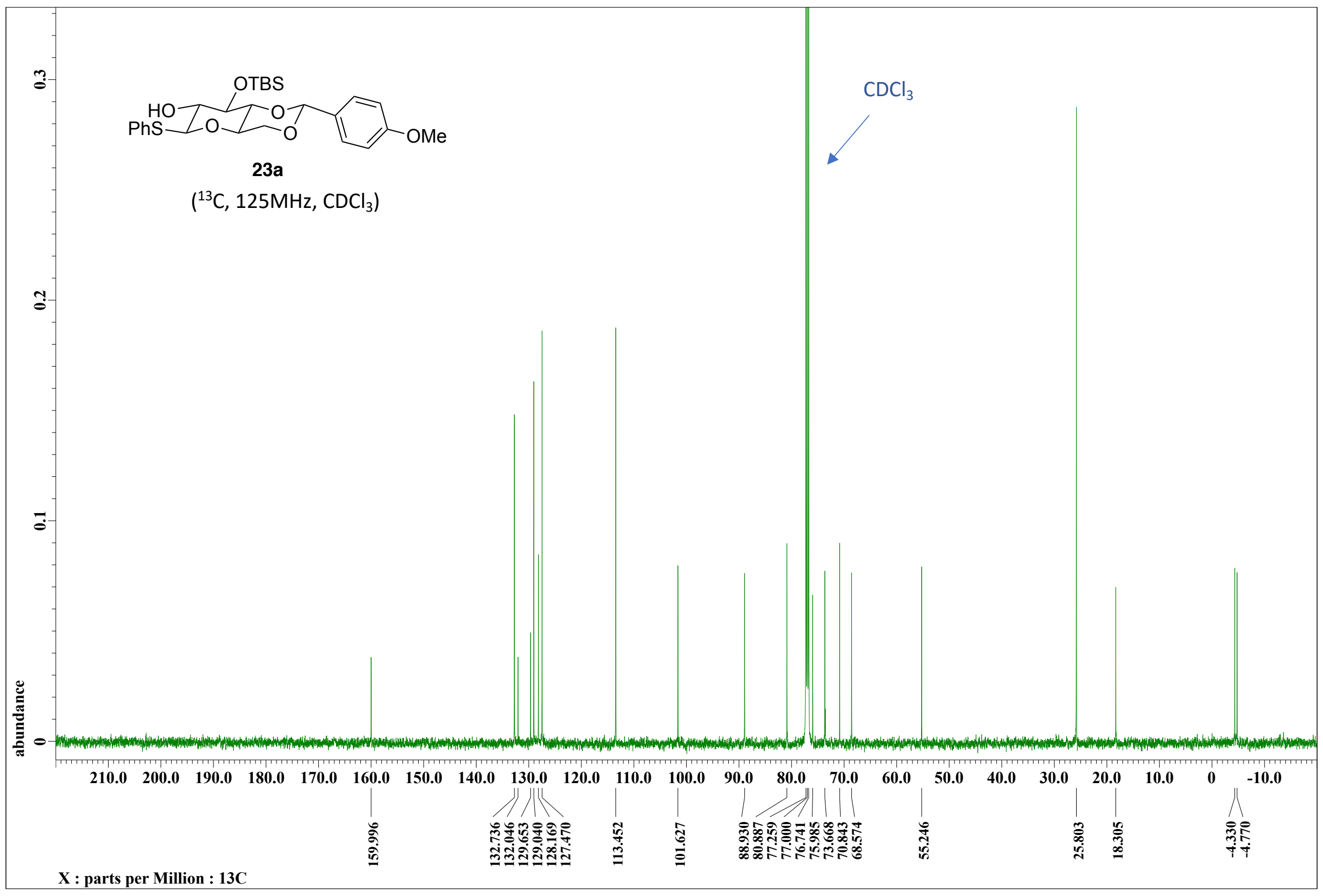




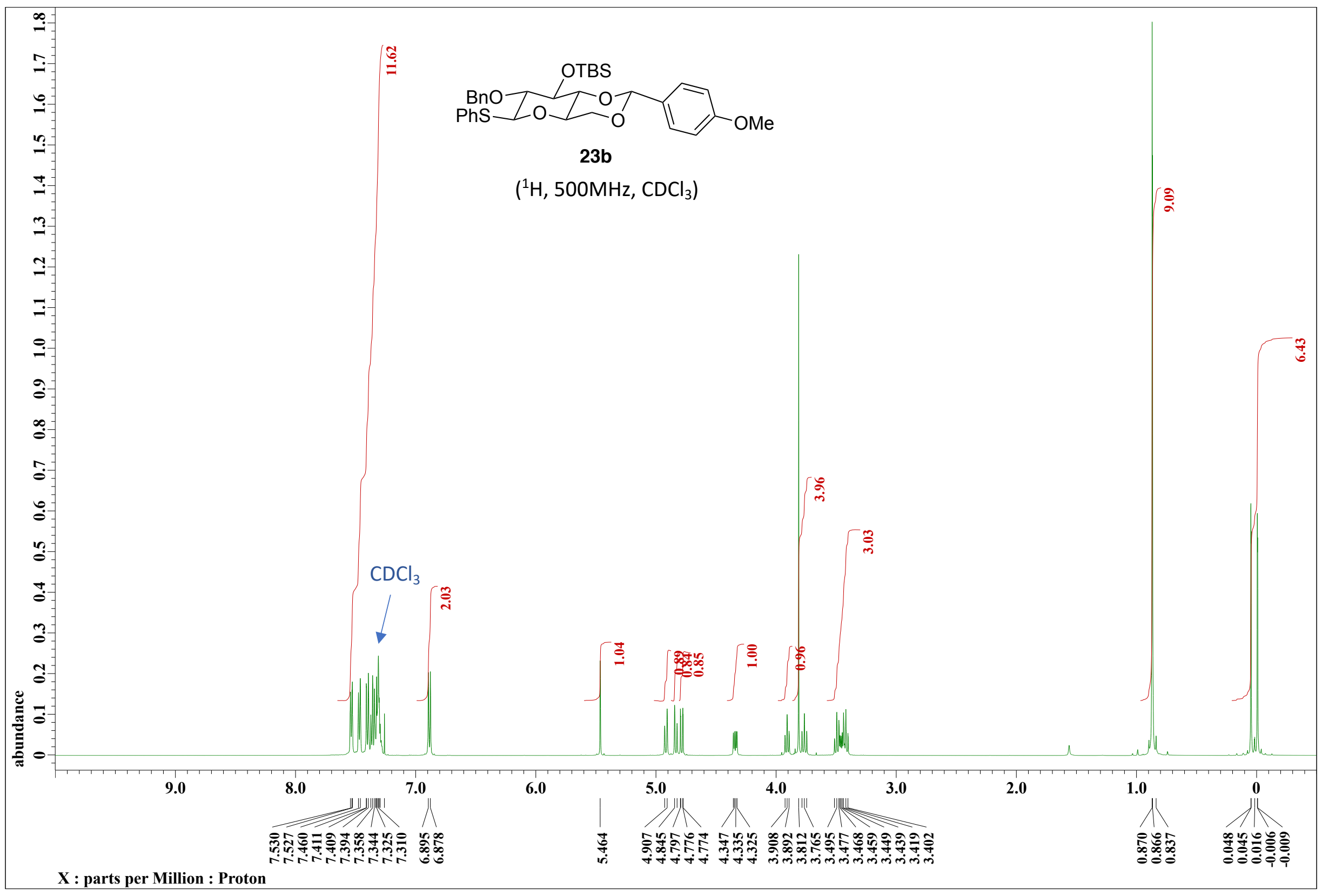




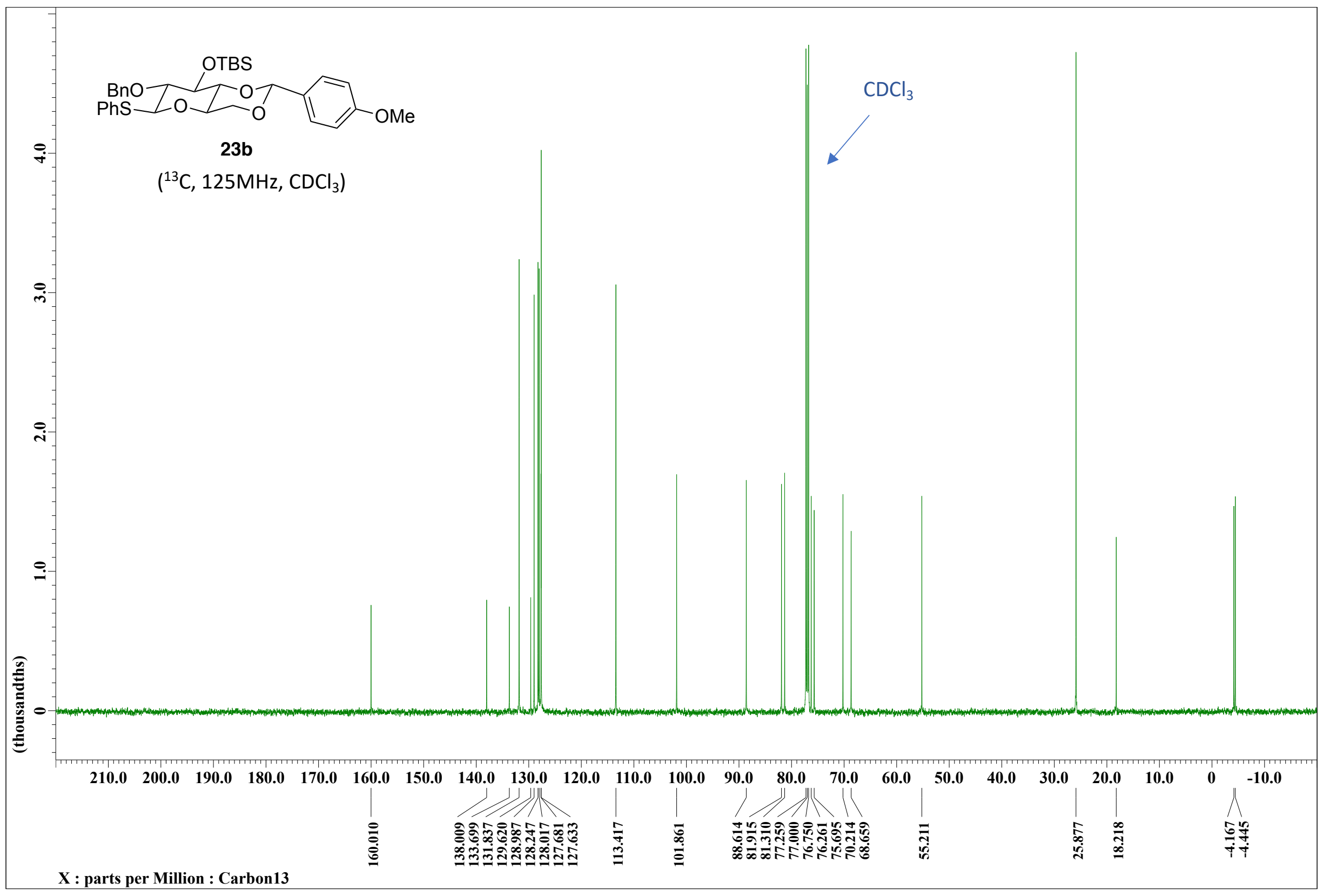




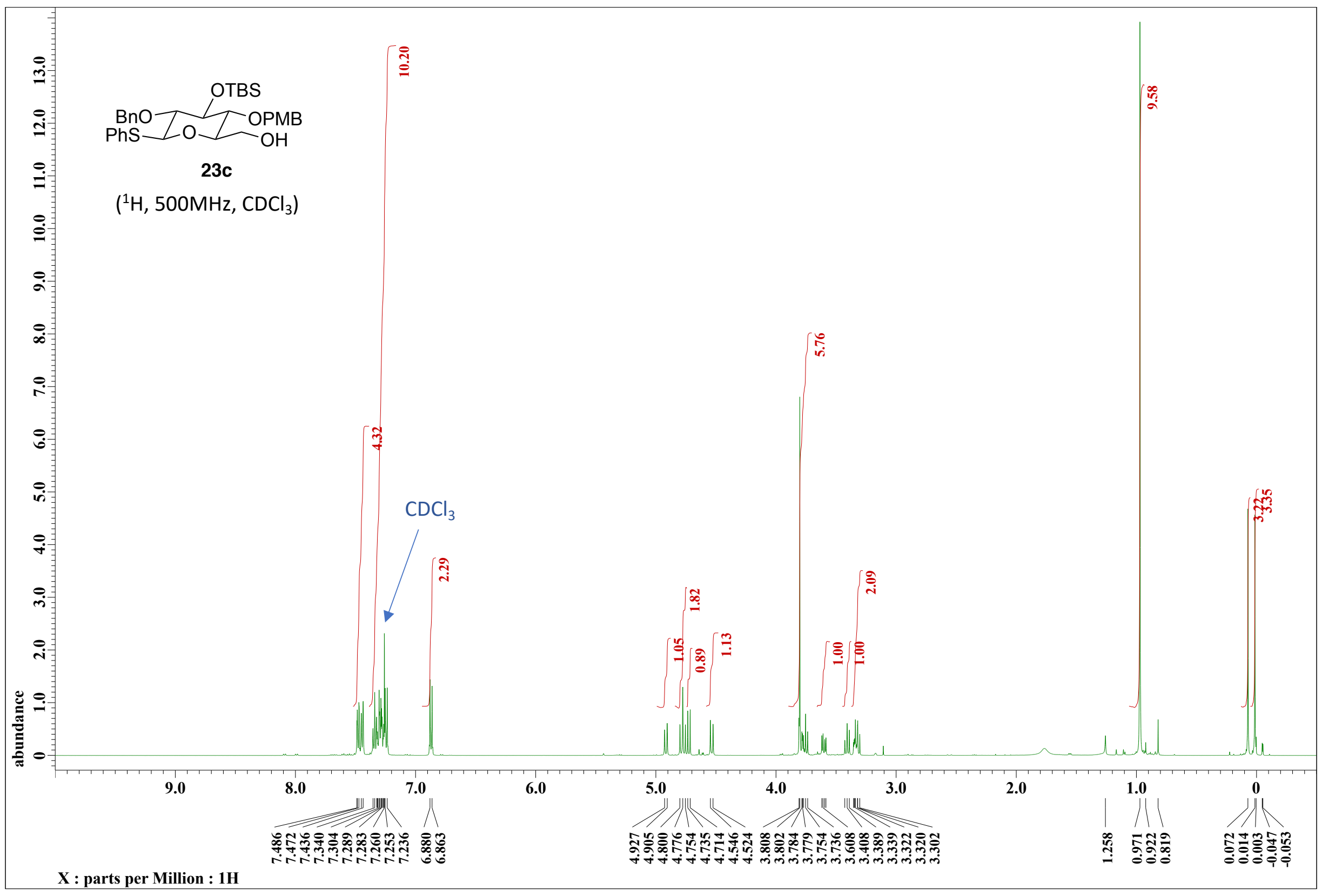




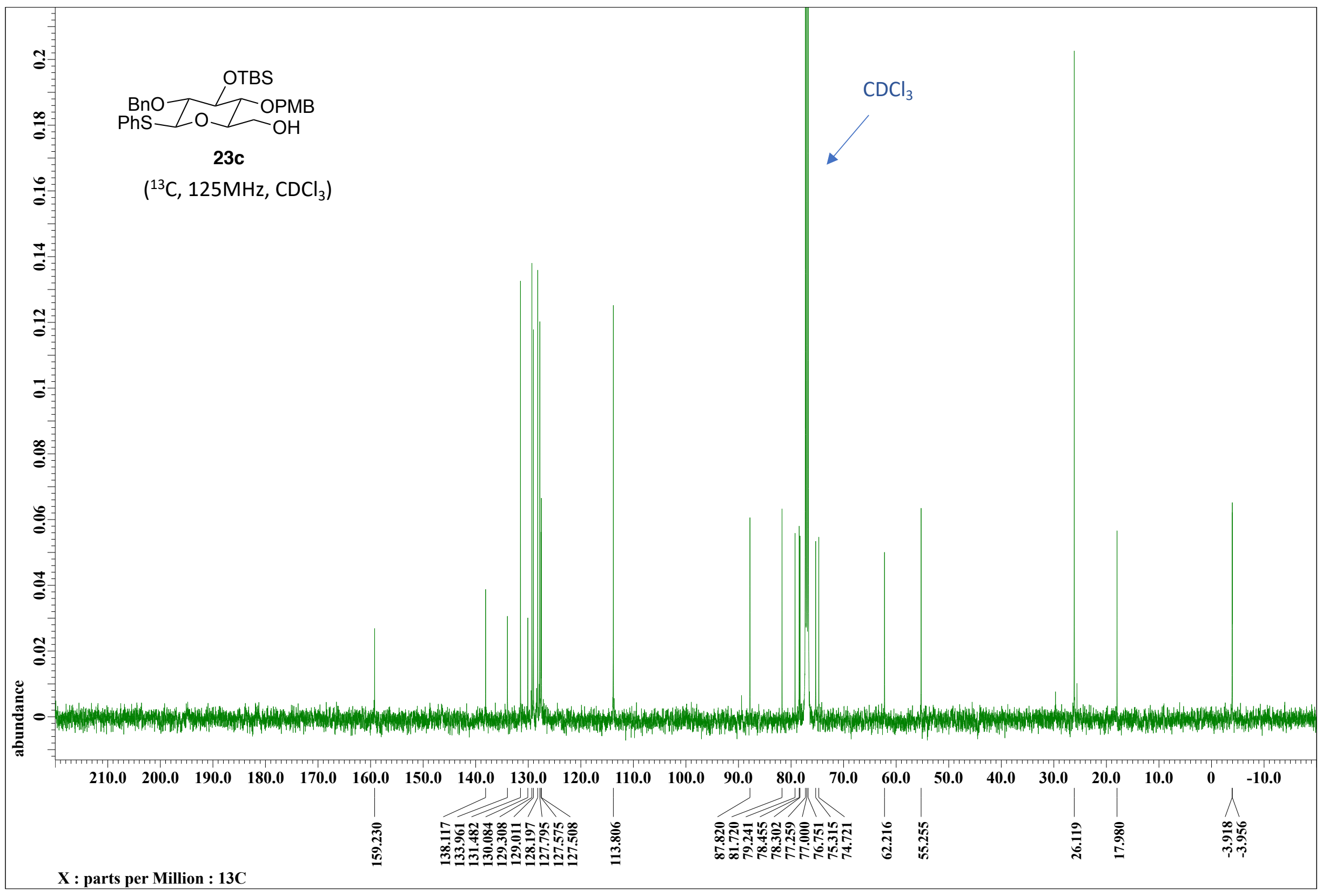




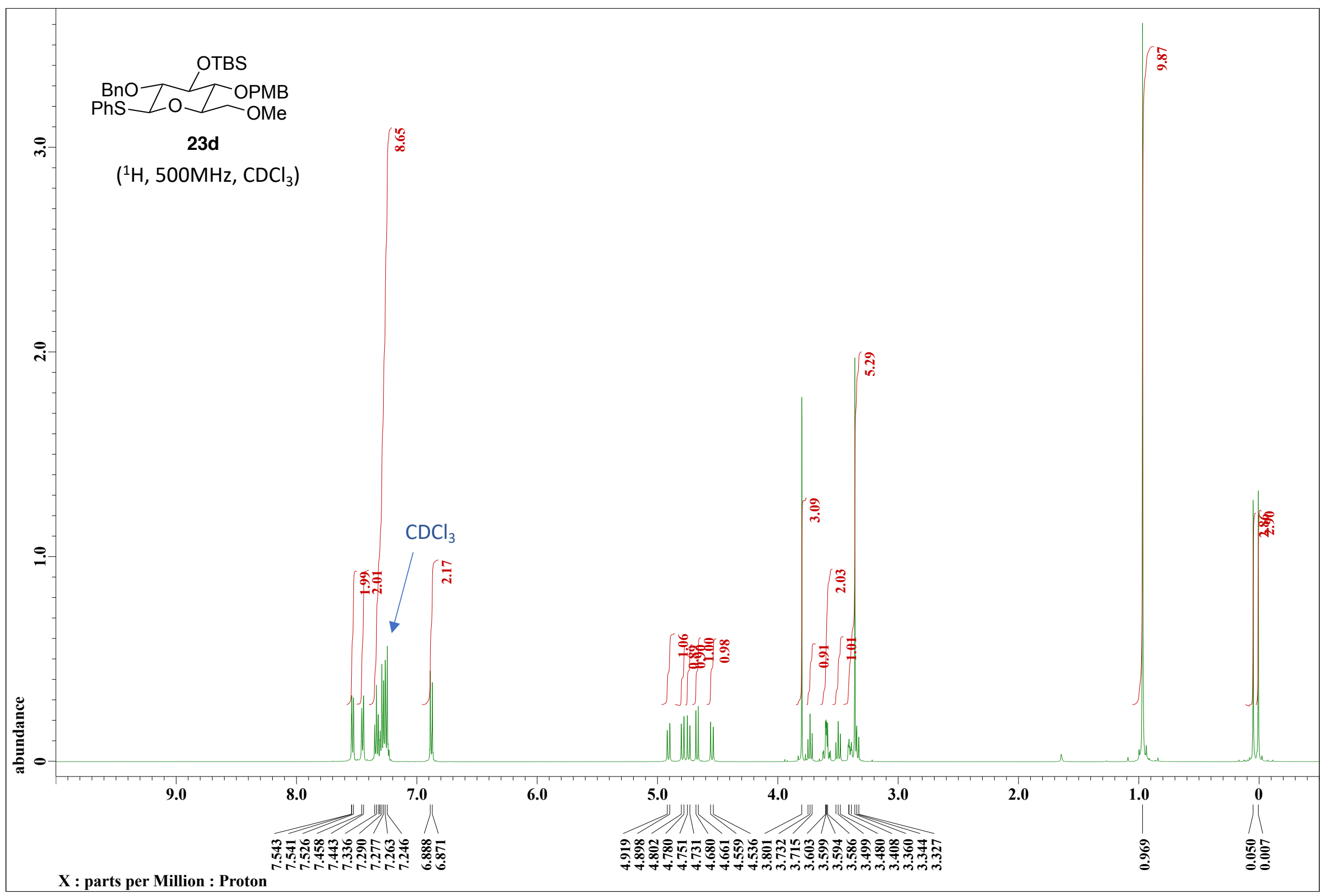




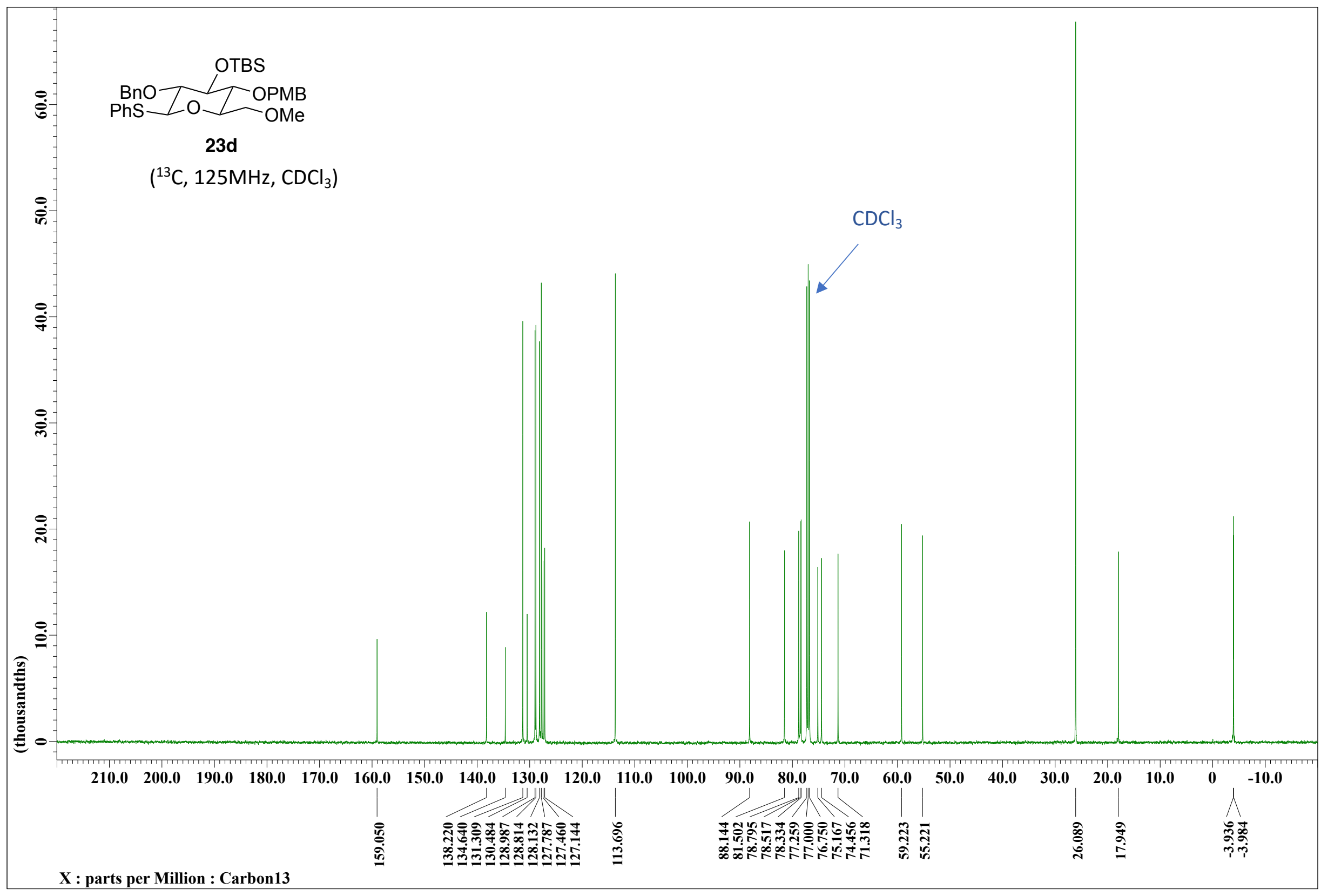




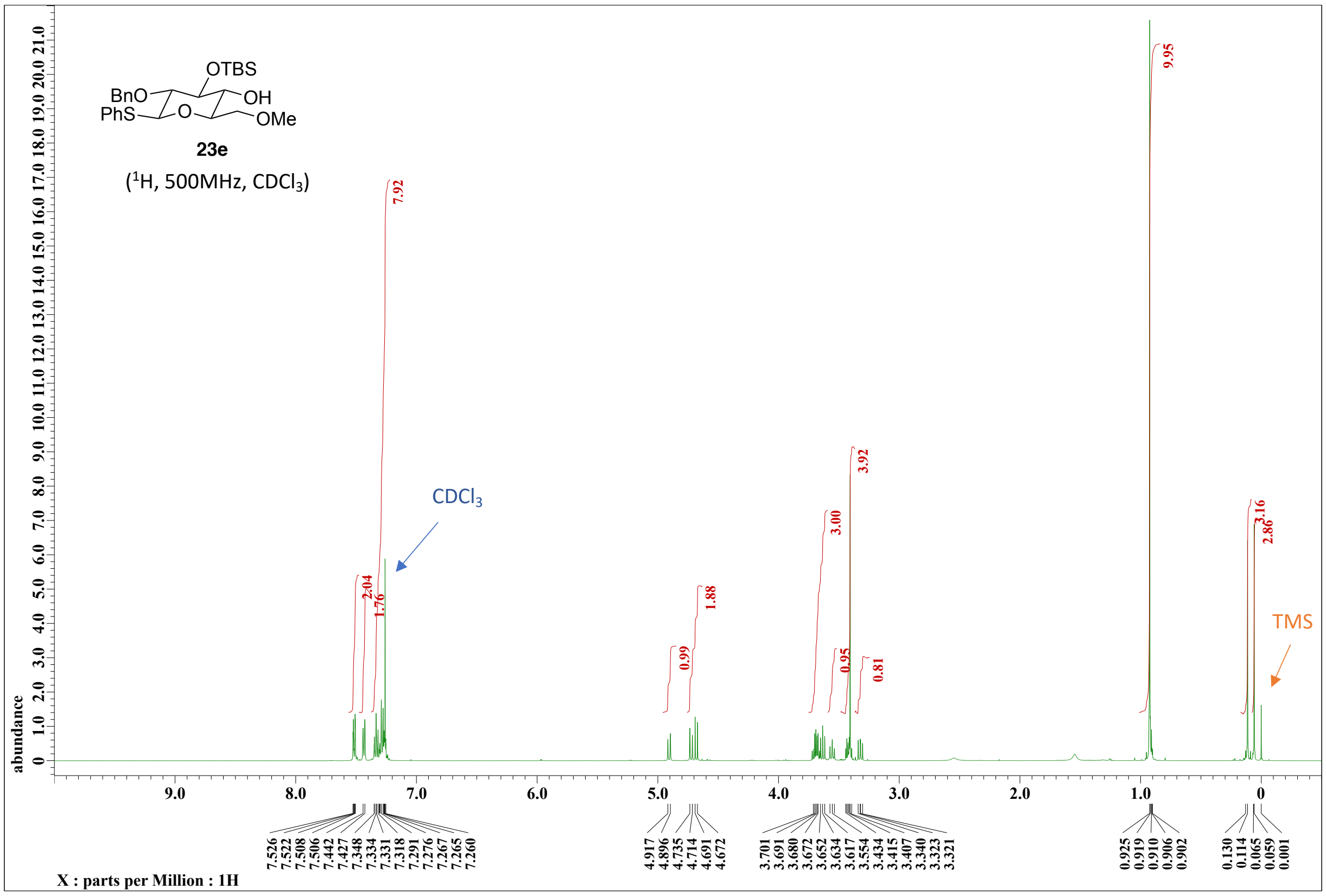




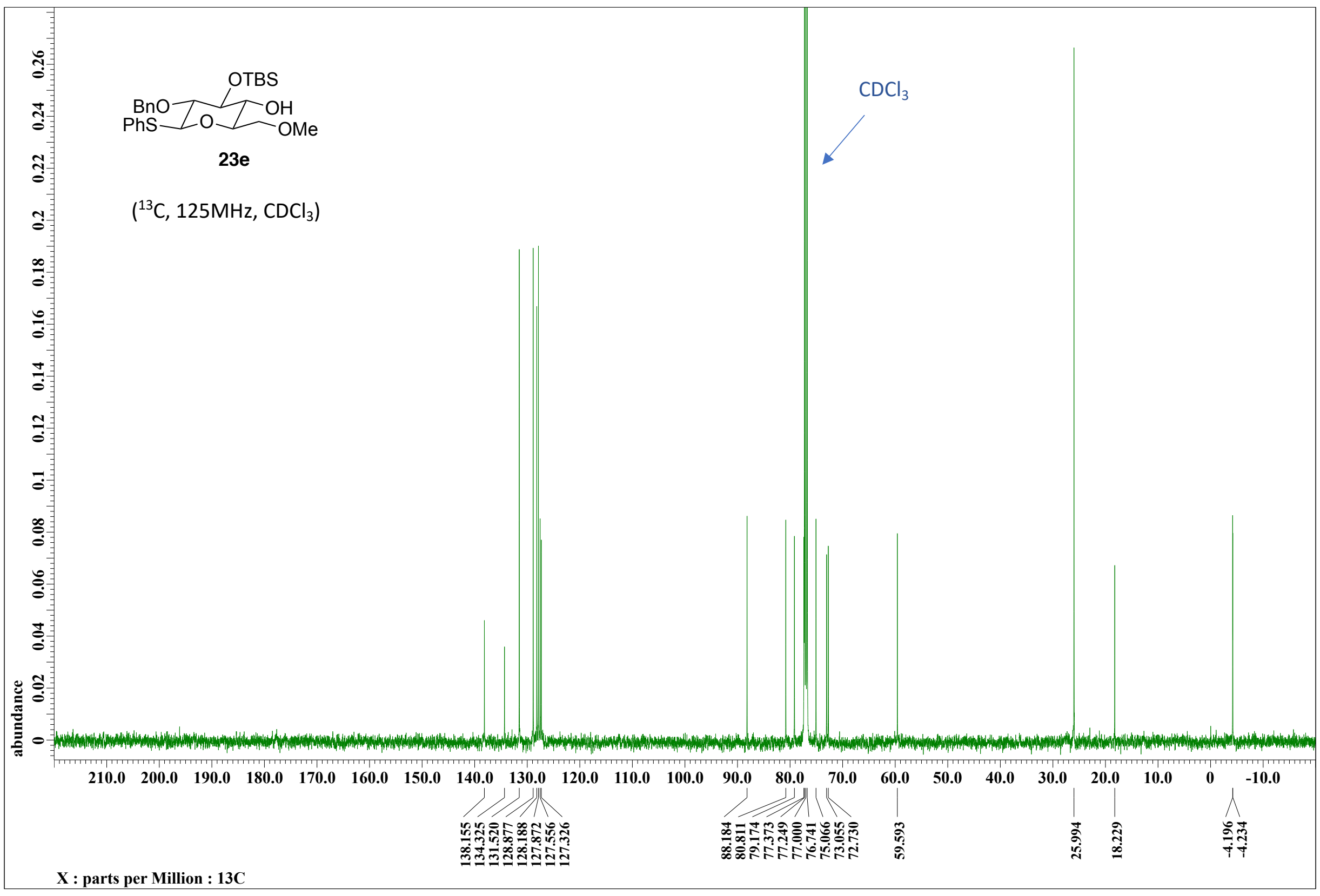




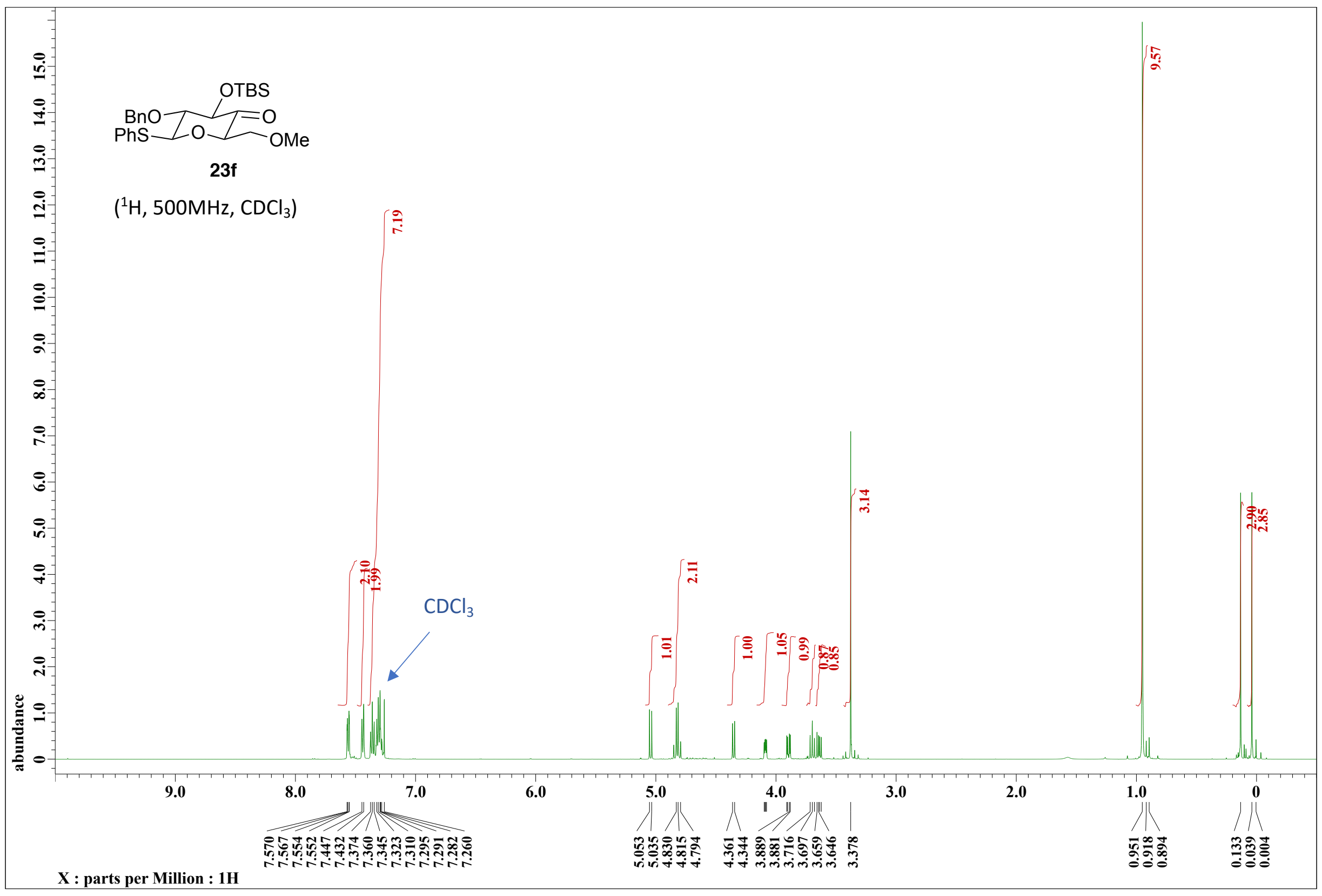




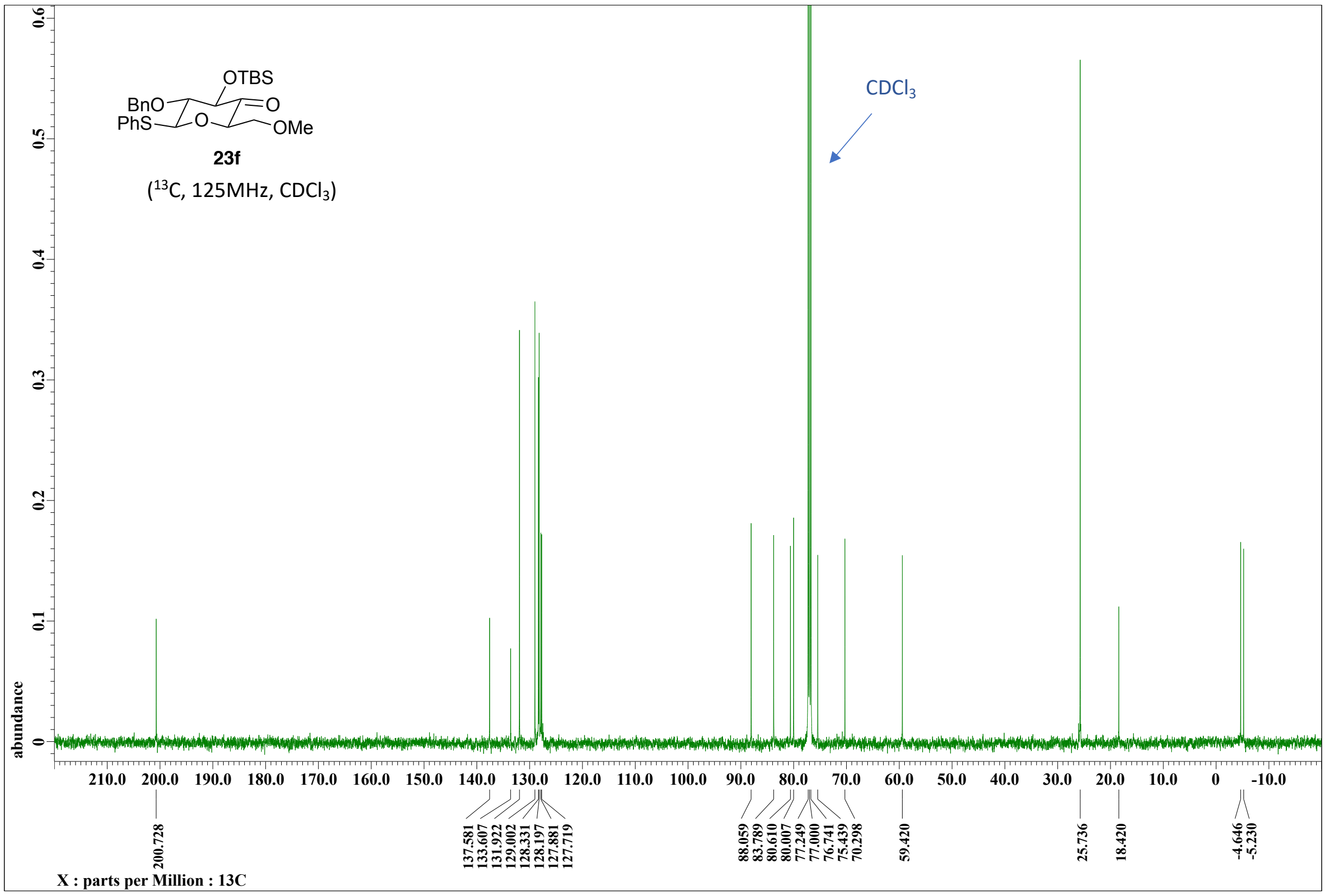




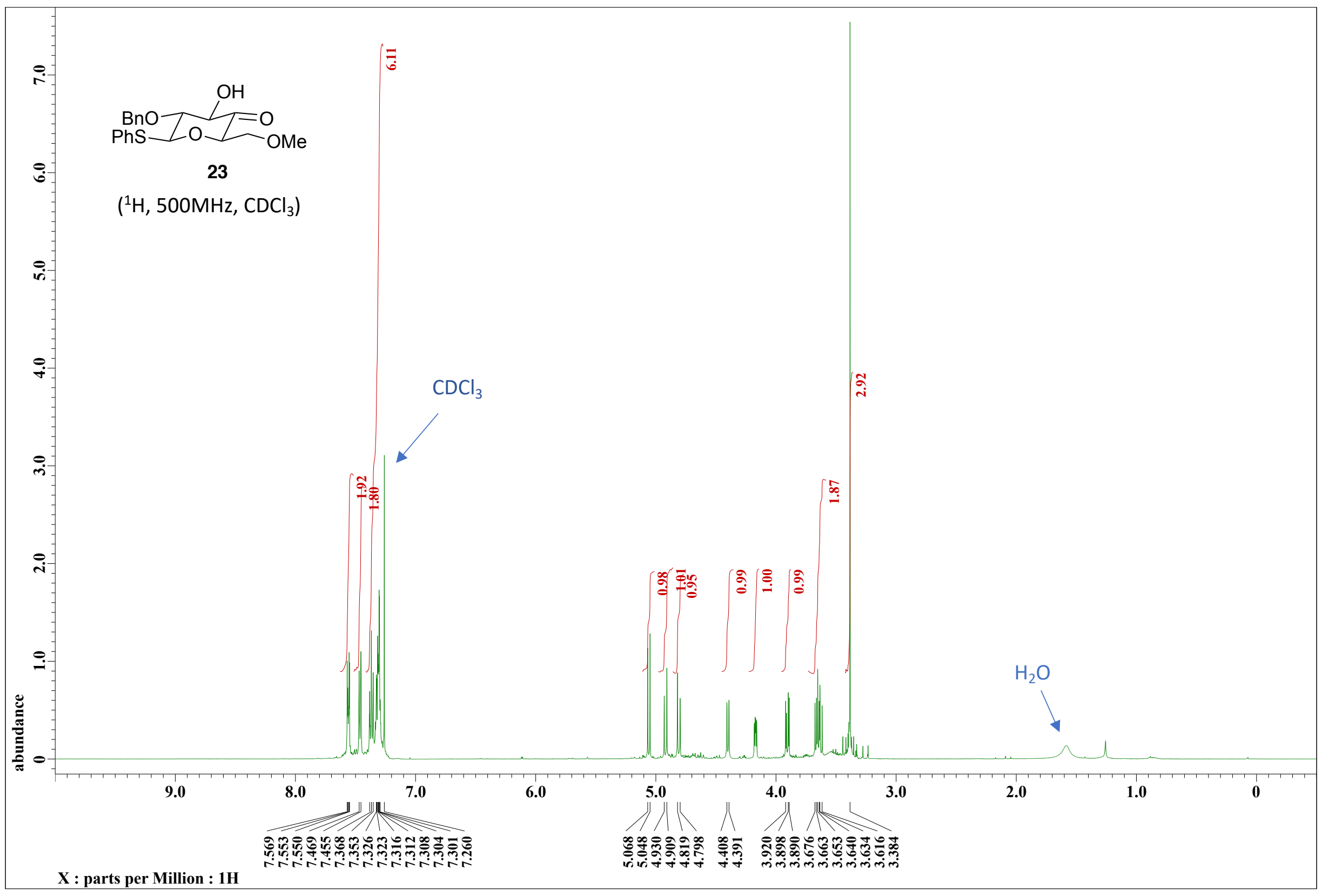




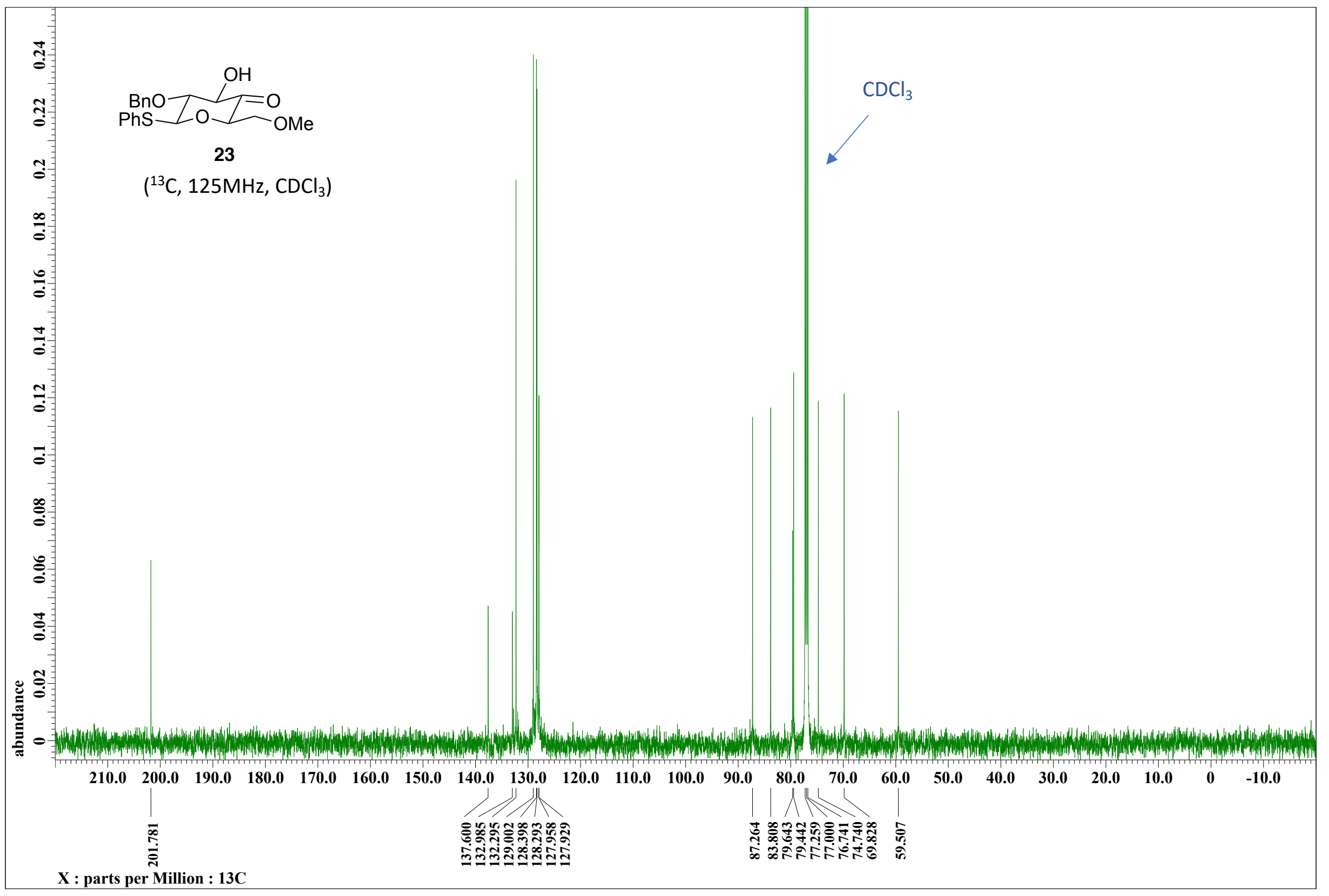




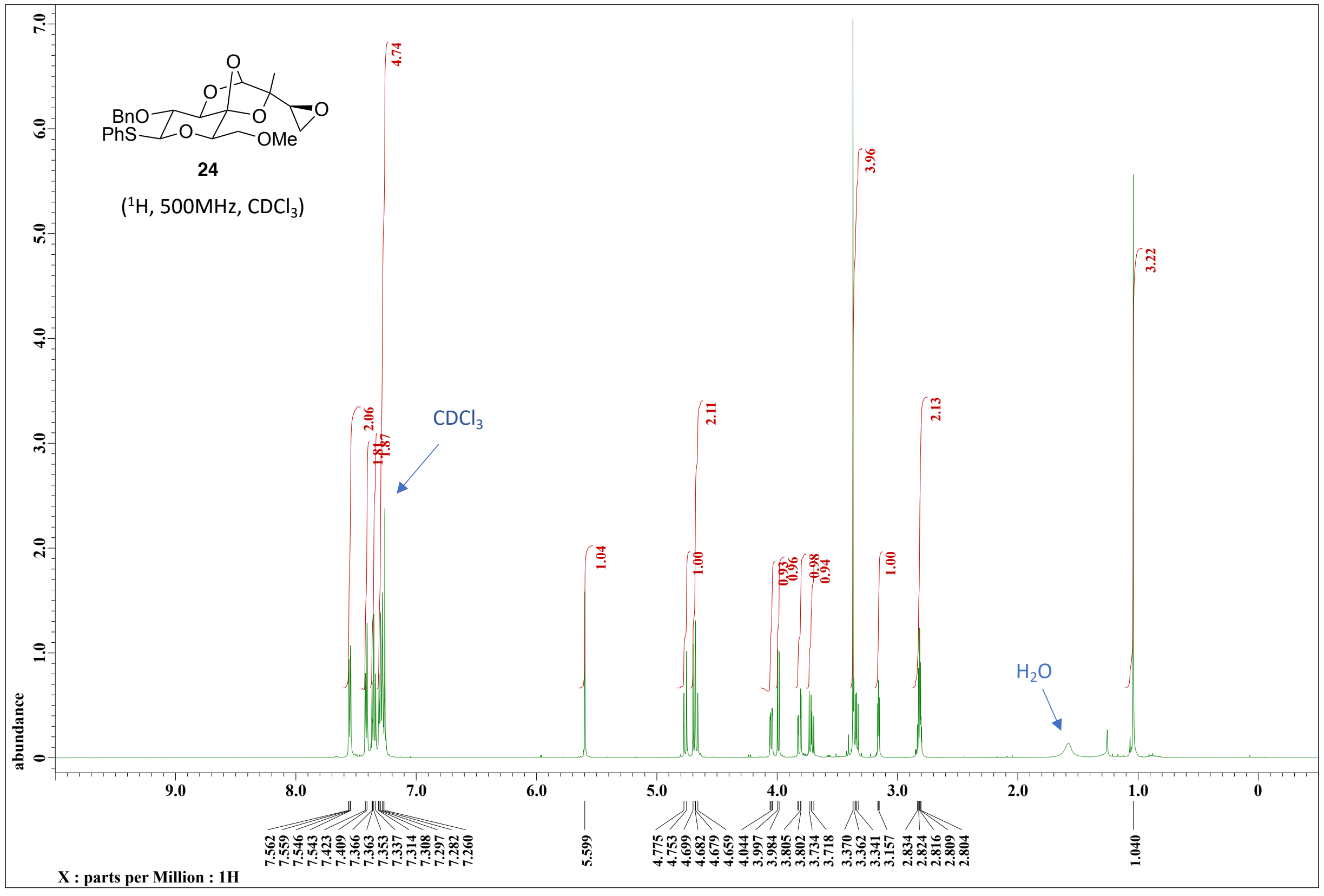




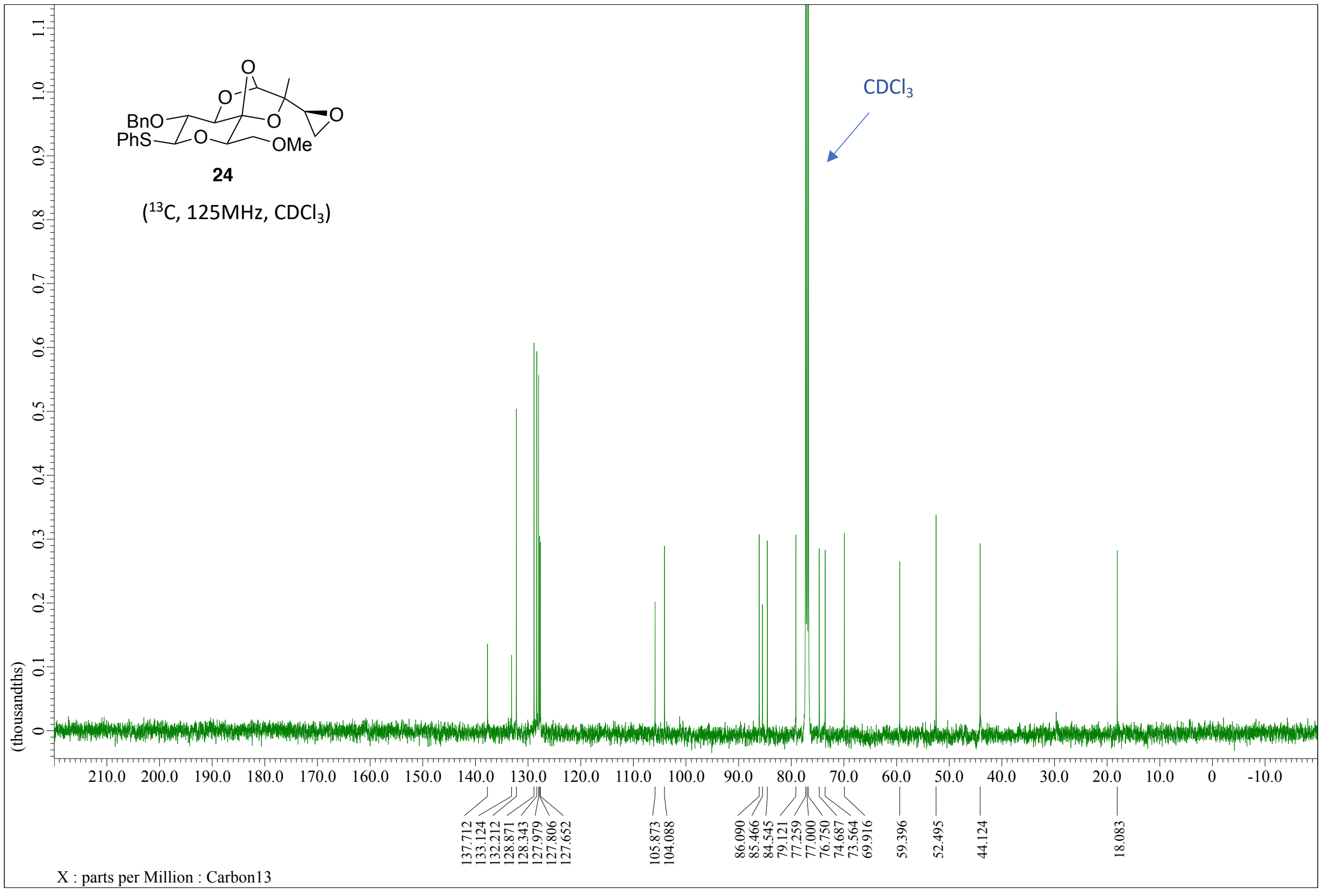




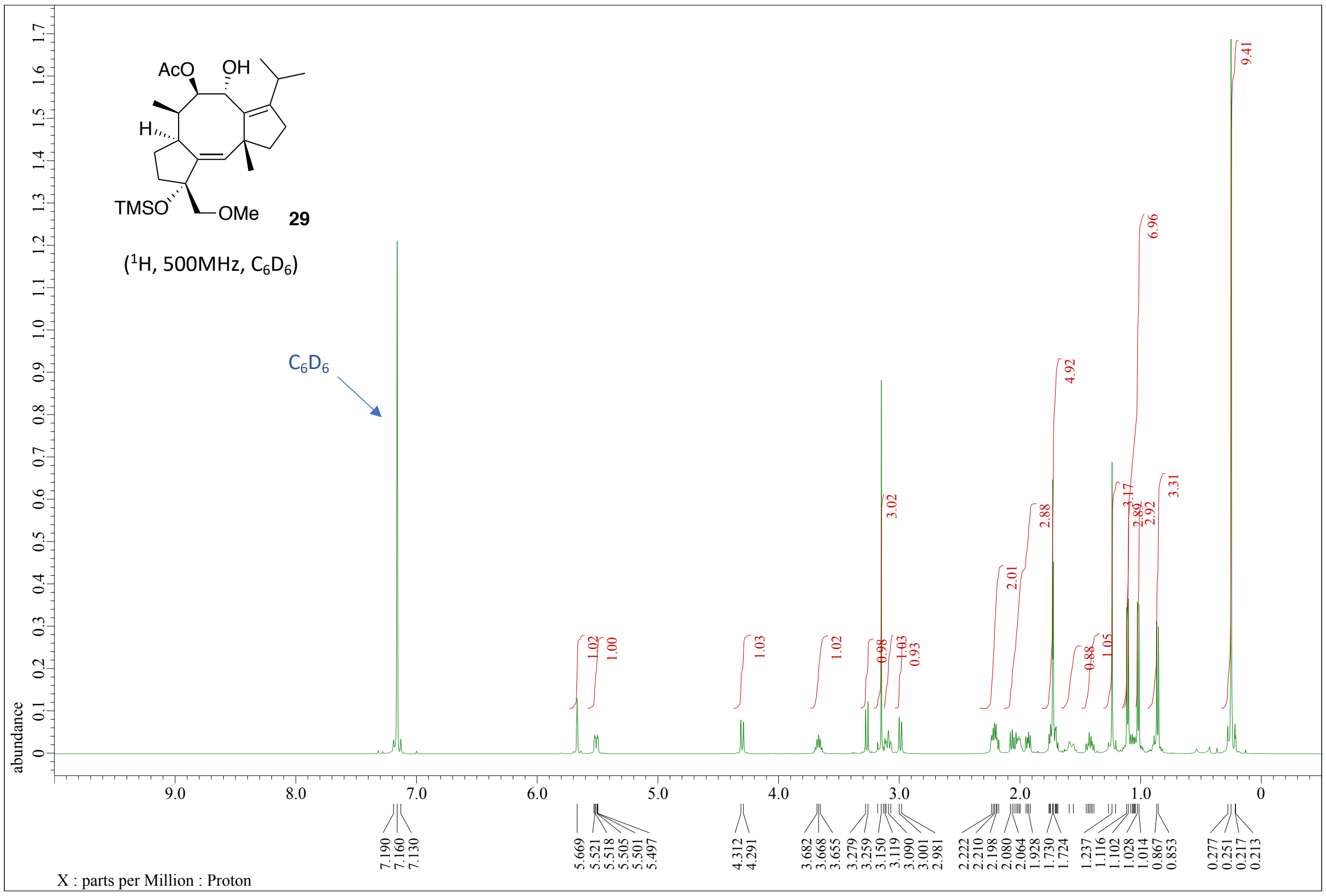




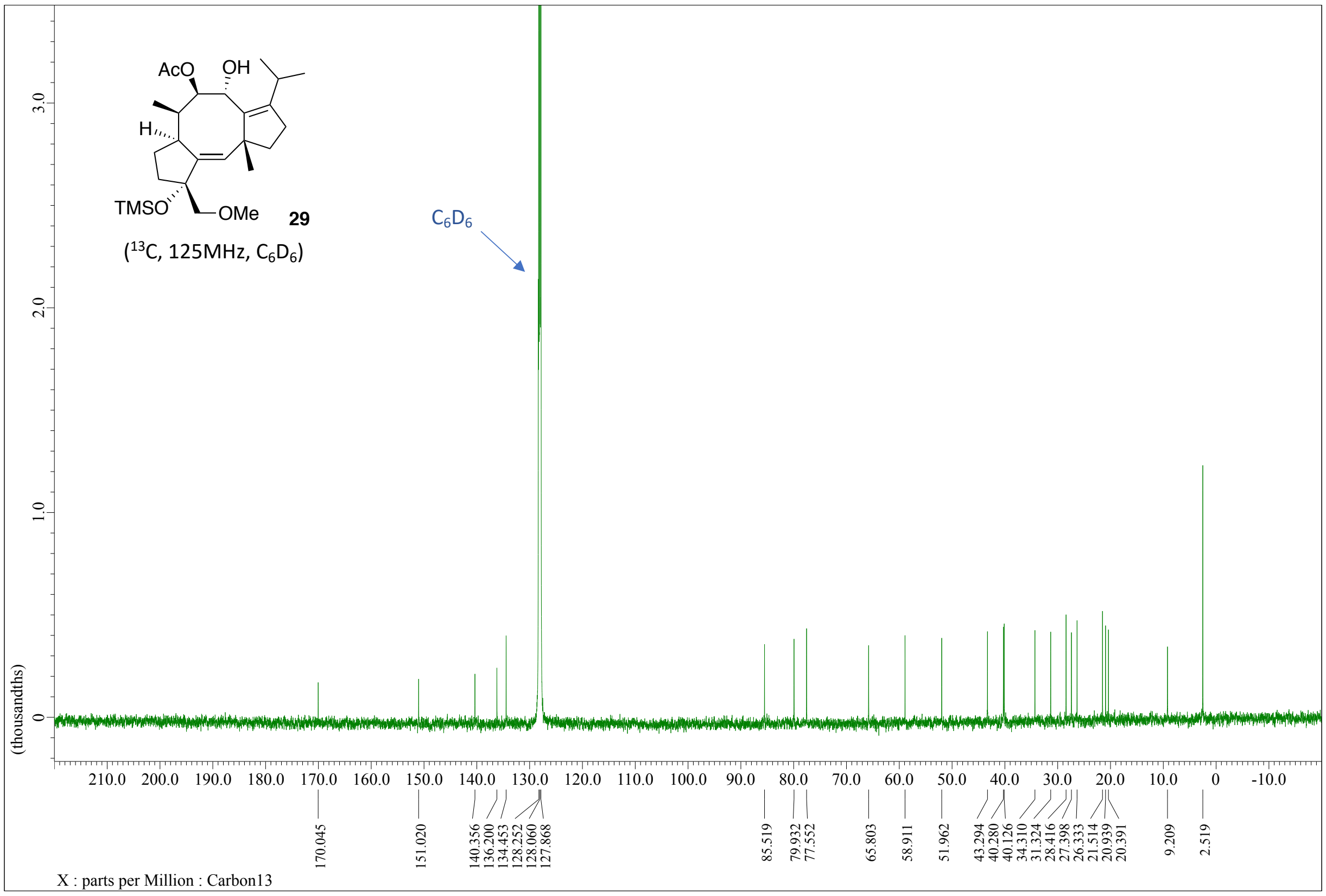




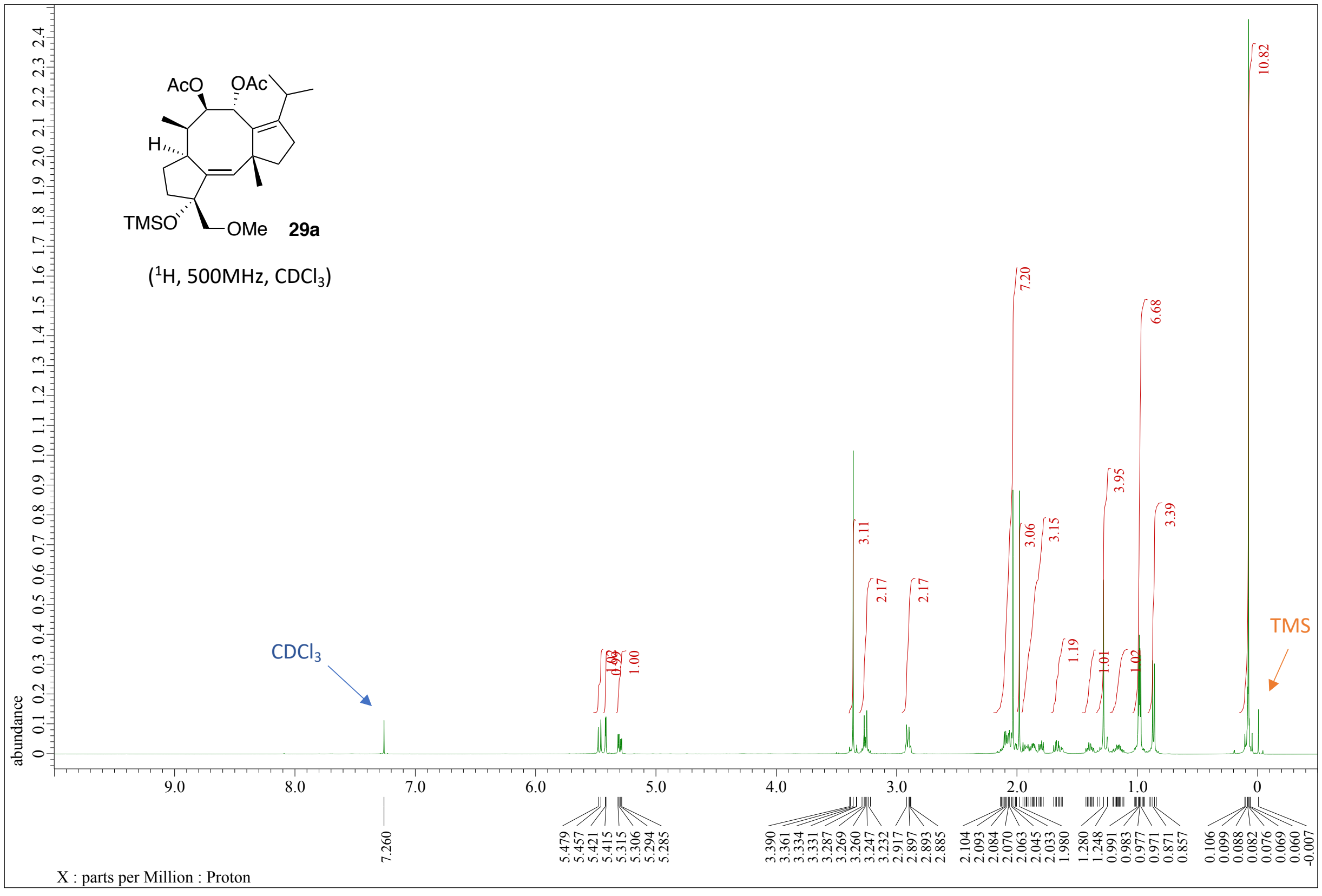




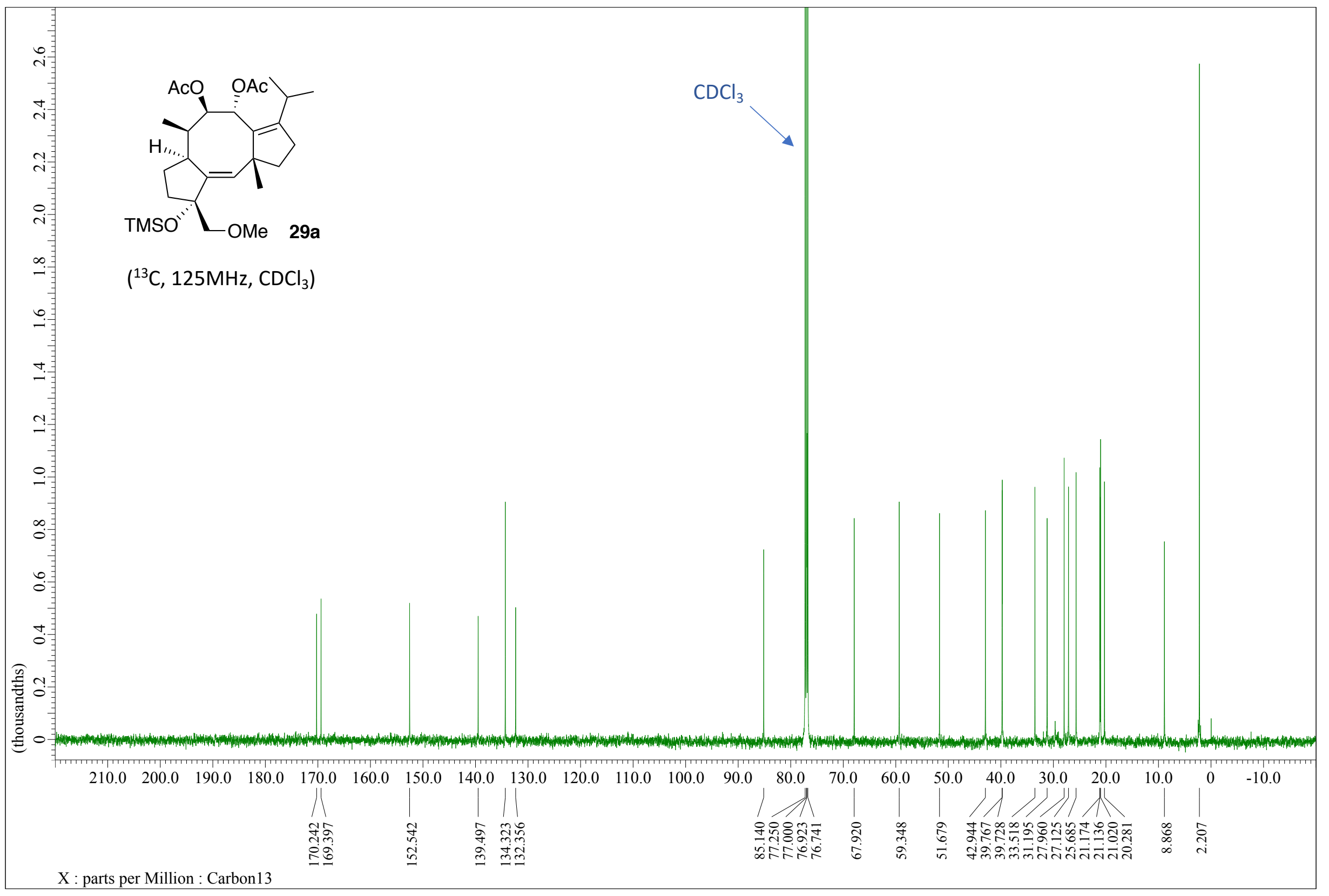




\section{0 to -0.5 ppm (24 + 29 to $\mathbf{3 0})$}

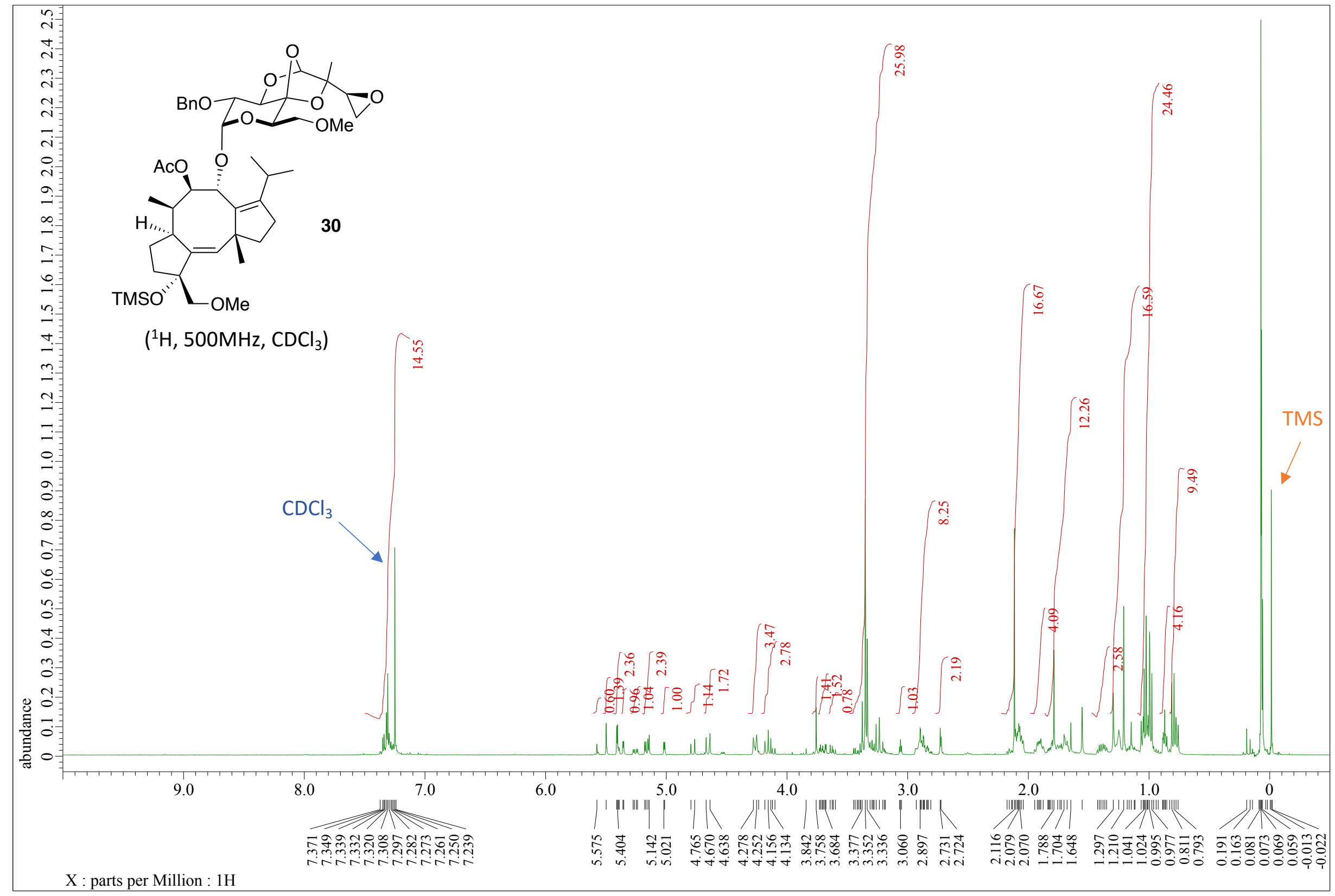




\section{8 to 2.5 ppm ( $24+29$ to 30$)$}

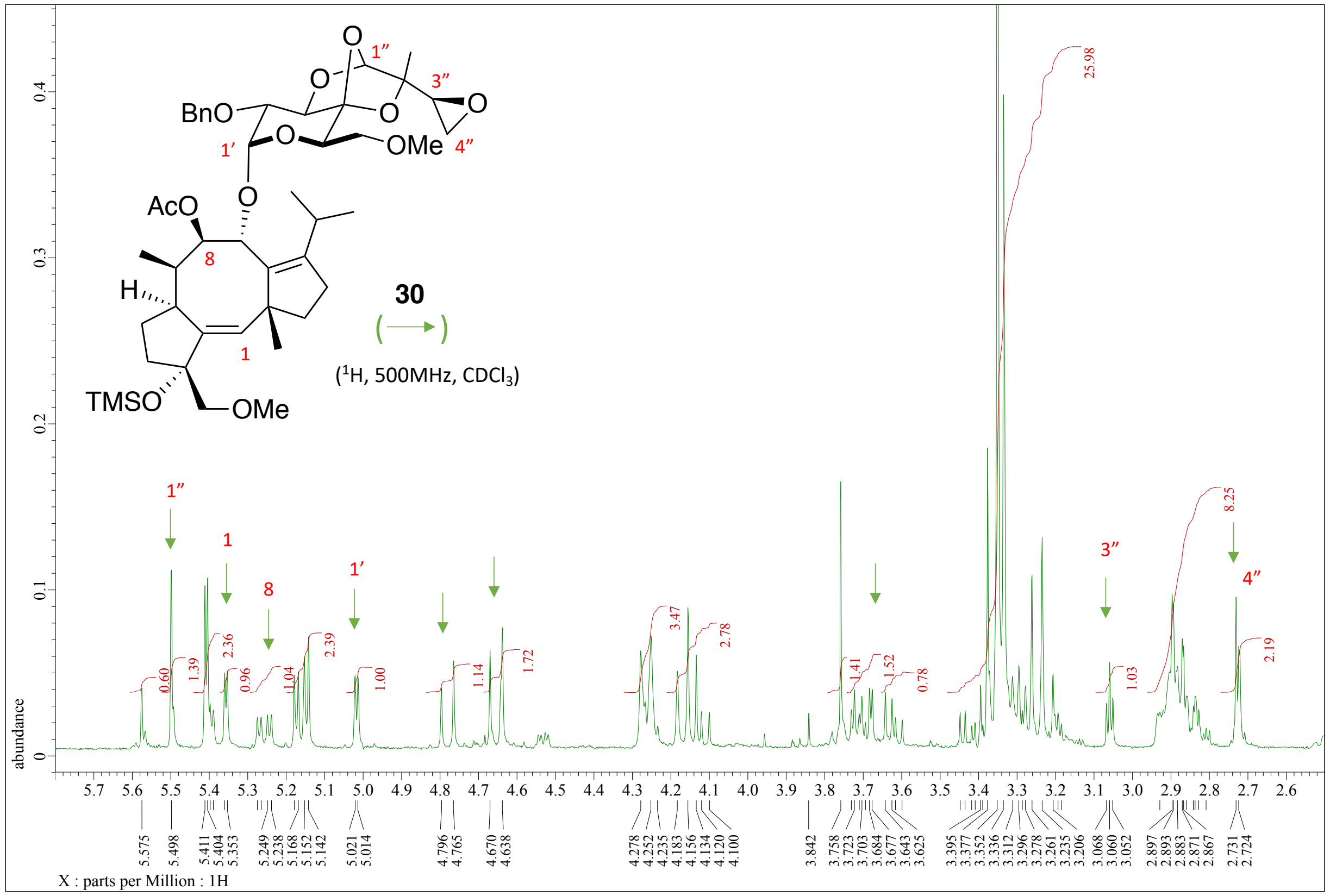


8.0 to $-0.5 \mathrm{ppm}(\mathbf{2 4}+\mathbf{2 9}$ to $\mathbf{3 0})\left({ }^{1} \mathrm{H}, 500 \mathrm{MHz}, \mathrm{CDCl}_{3}\right)$

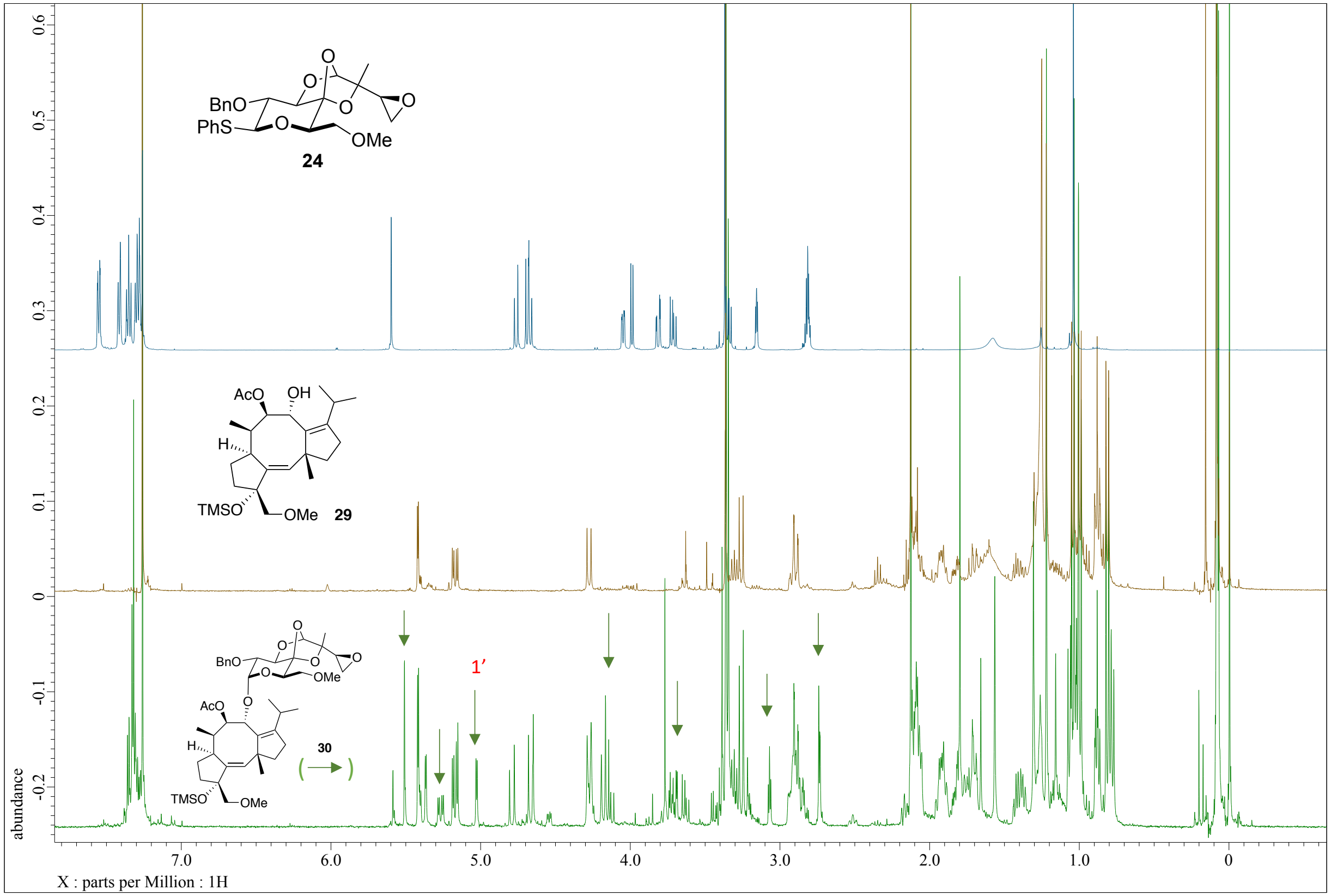


6.0 to 2.5 ppm $(24+29$ to 30$)\left({ }^{1} \mathrm{H}, 500 \mathrm{MHz}, \mathrm{CDCl}_{3}\right)$

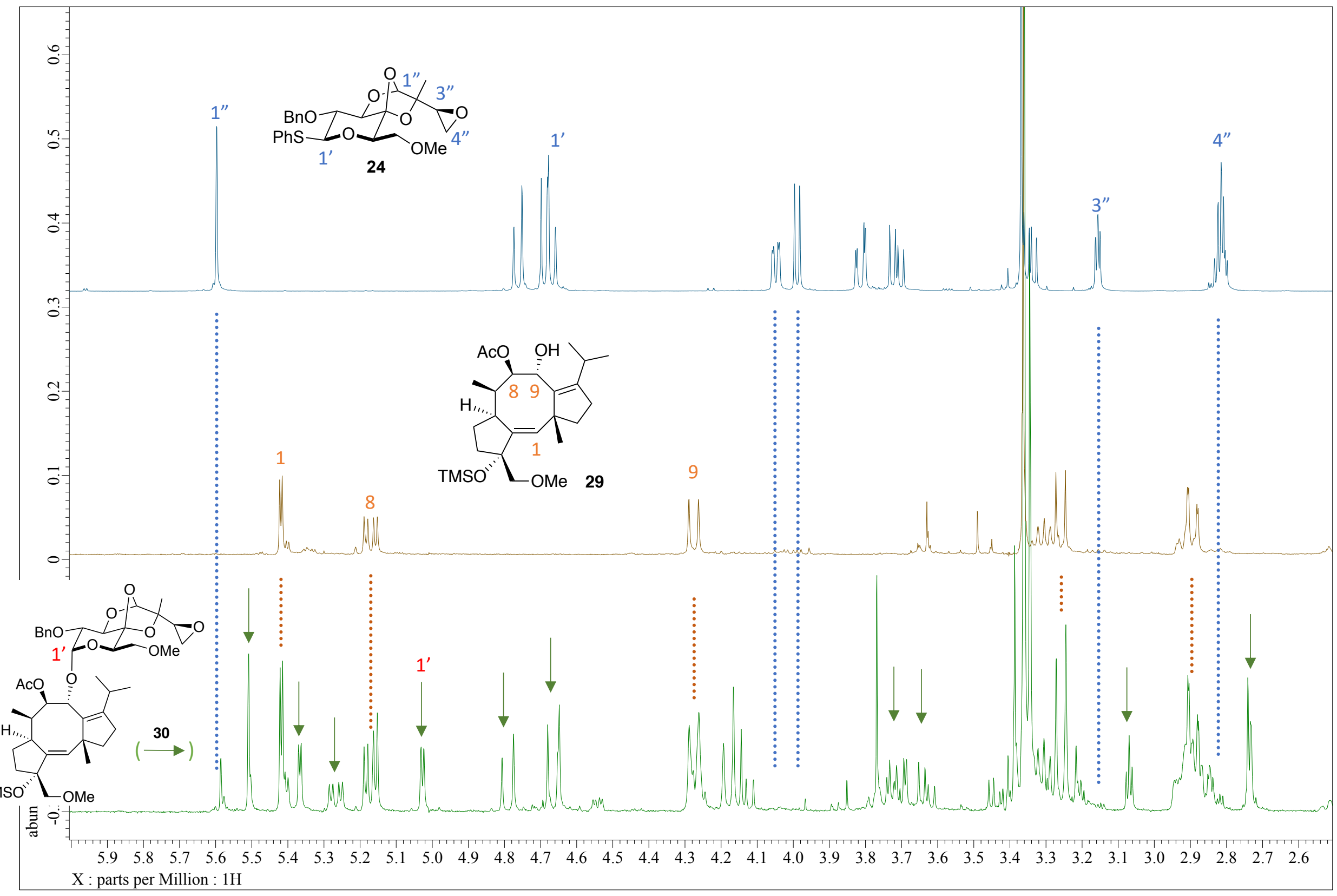


10.0 to -0.5 ppm (32 + inseparable unknown impurities)

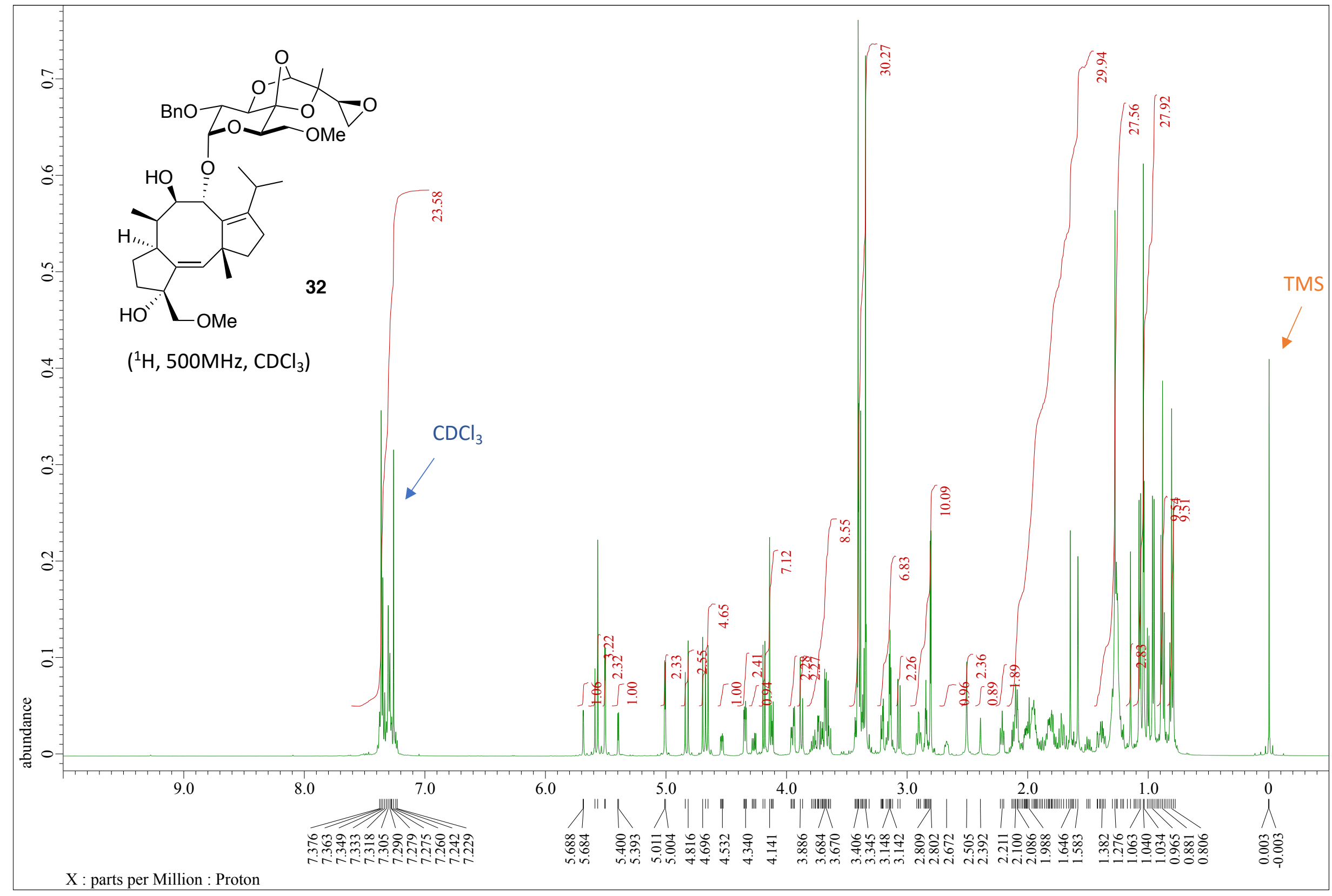


6.0 to 2.3 ppm ( 32 + inseparable unknown impurities)

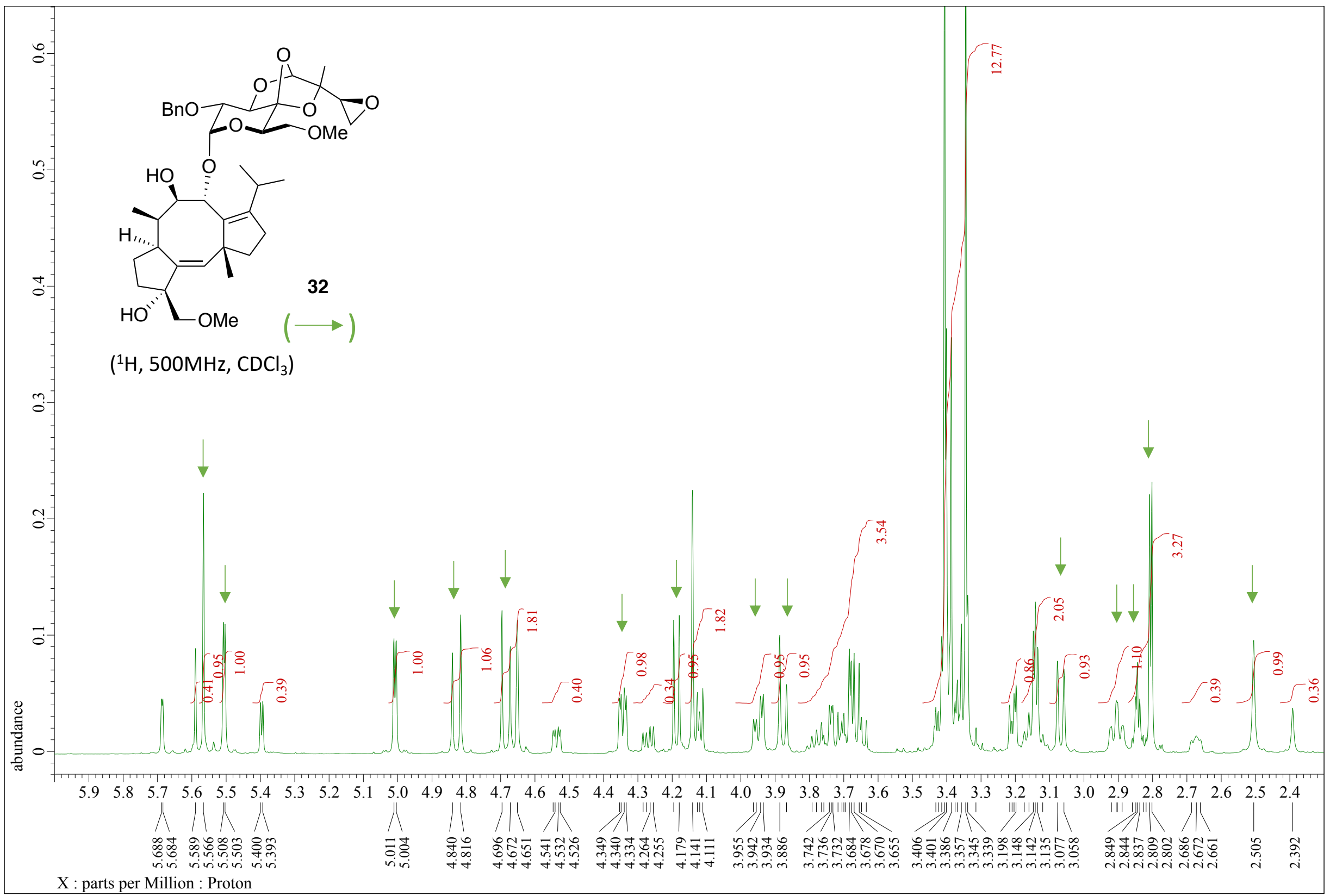


10.0 to -0.5 ppm (32 + inseparable unknown impurities)

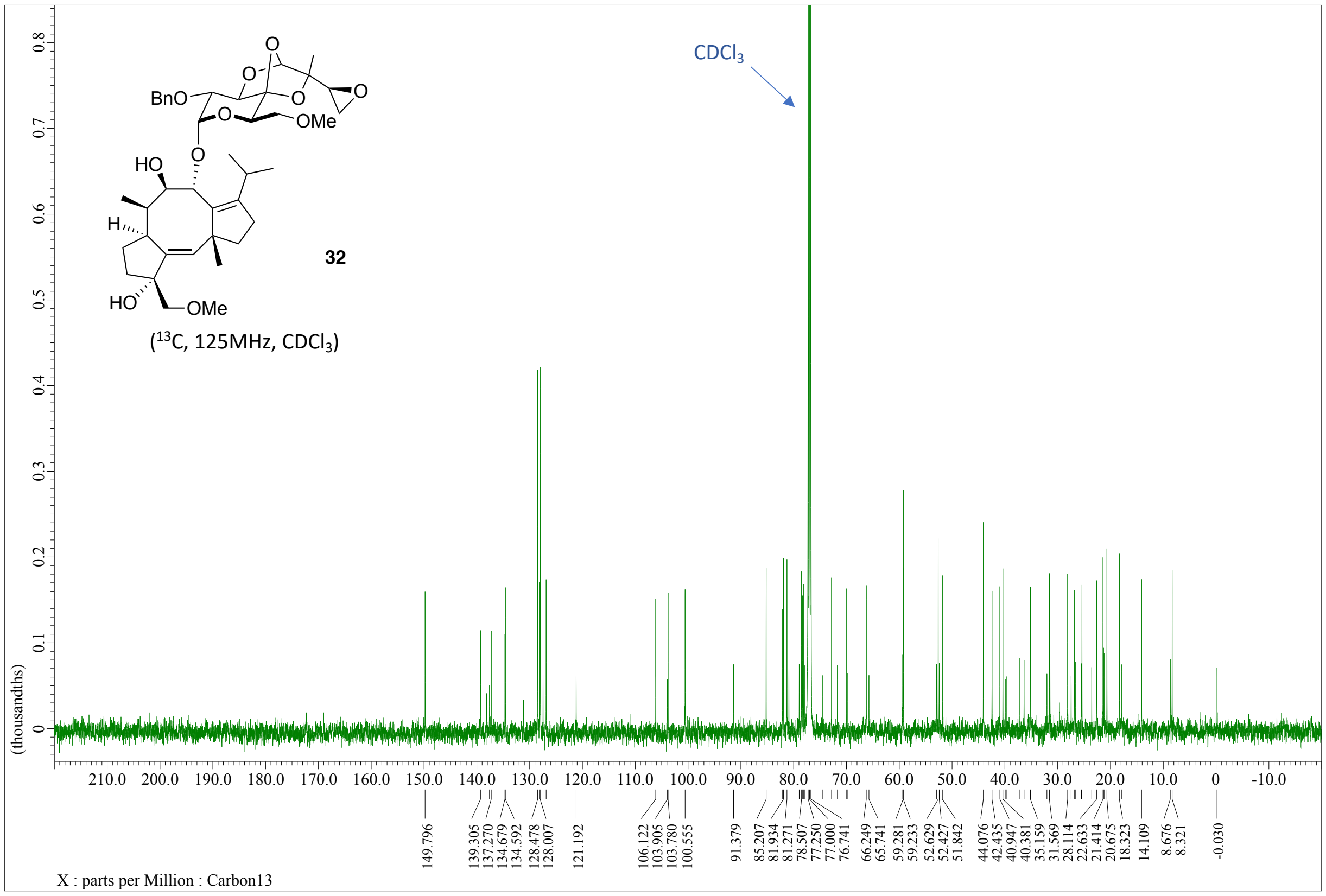


10.0 to $-0.5 \mathrm{ppm}$ (cotylenin A)

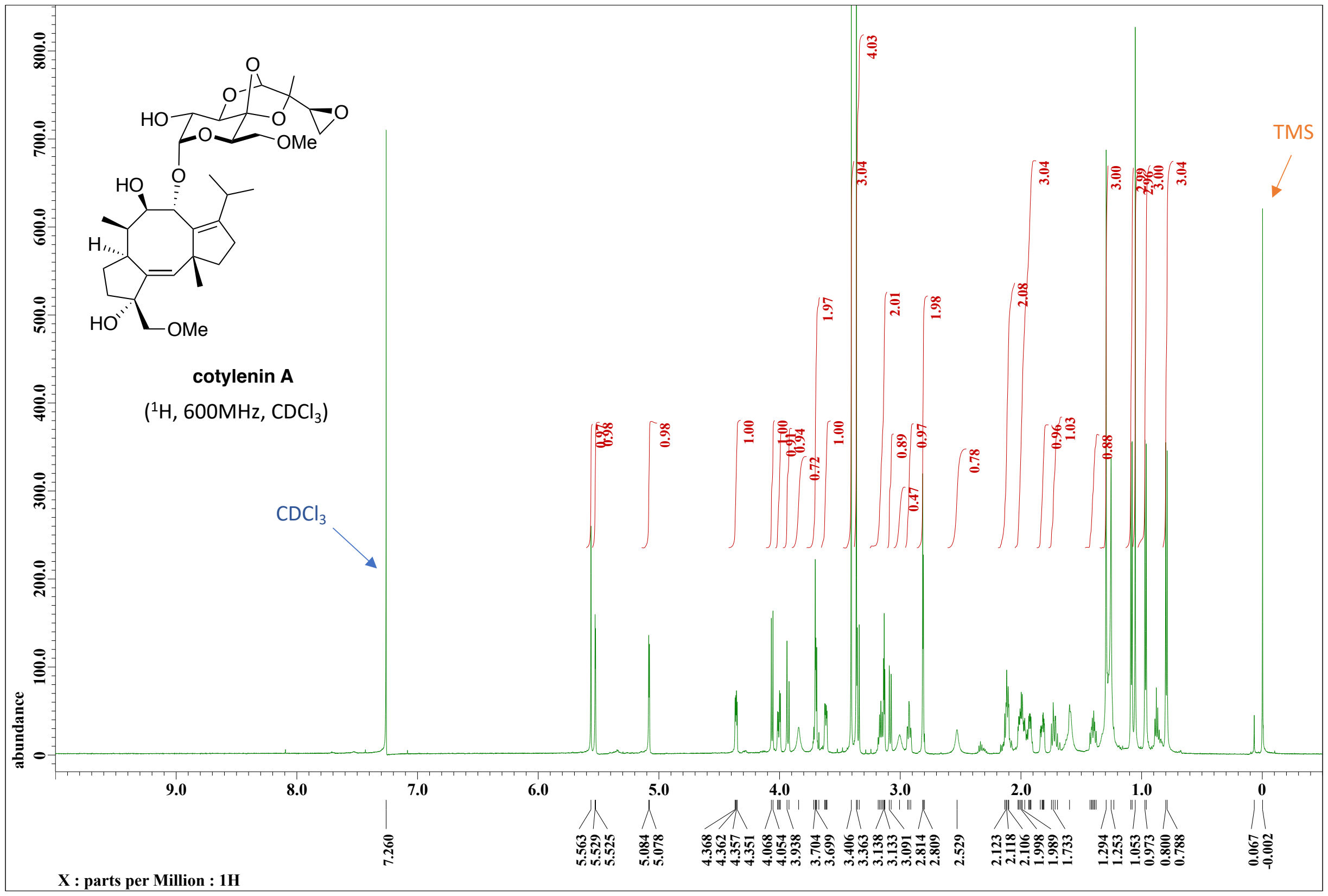


6.0 to $0.5 \mathrm{ppm}$ (cotylenin A)

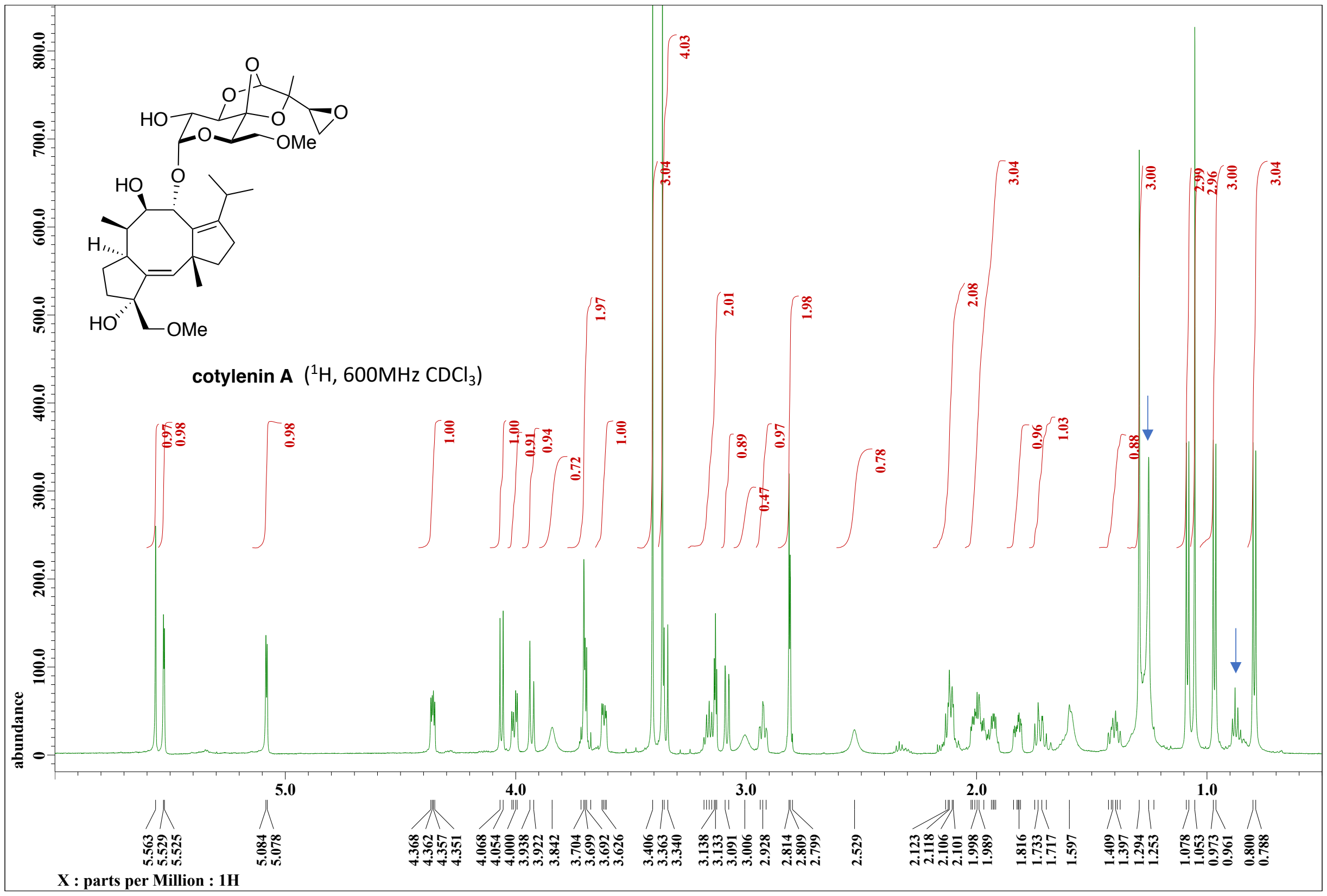

$\mathrm{X}$ : parts per Million : $1 \mathrm{H}$ 
220.0 to -20.0 ppm (cotylenin A)

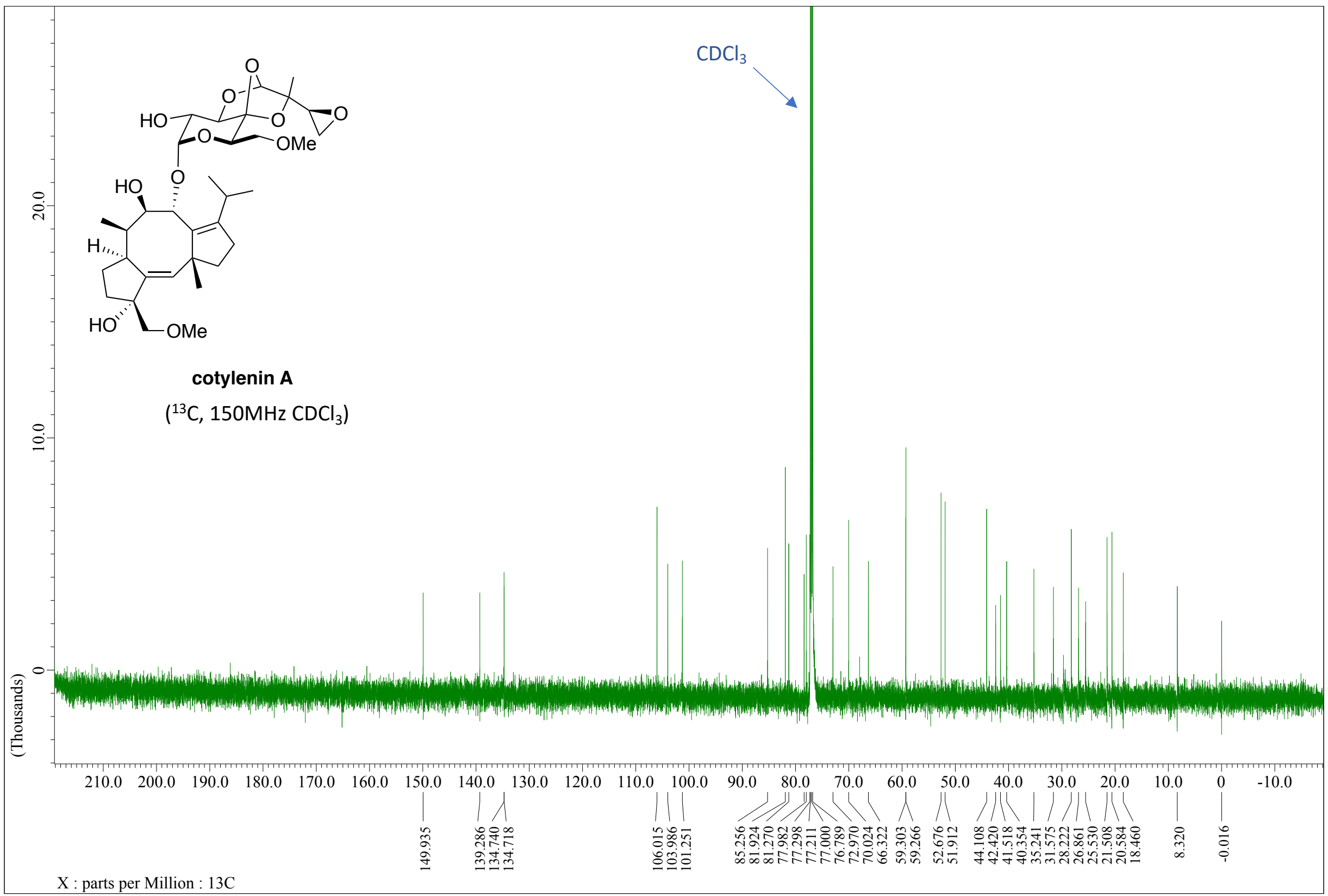


160.0 to $-10.0 \mathrm{ppm}$ (cotylenin A)

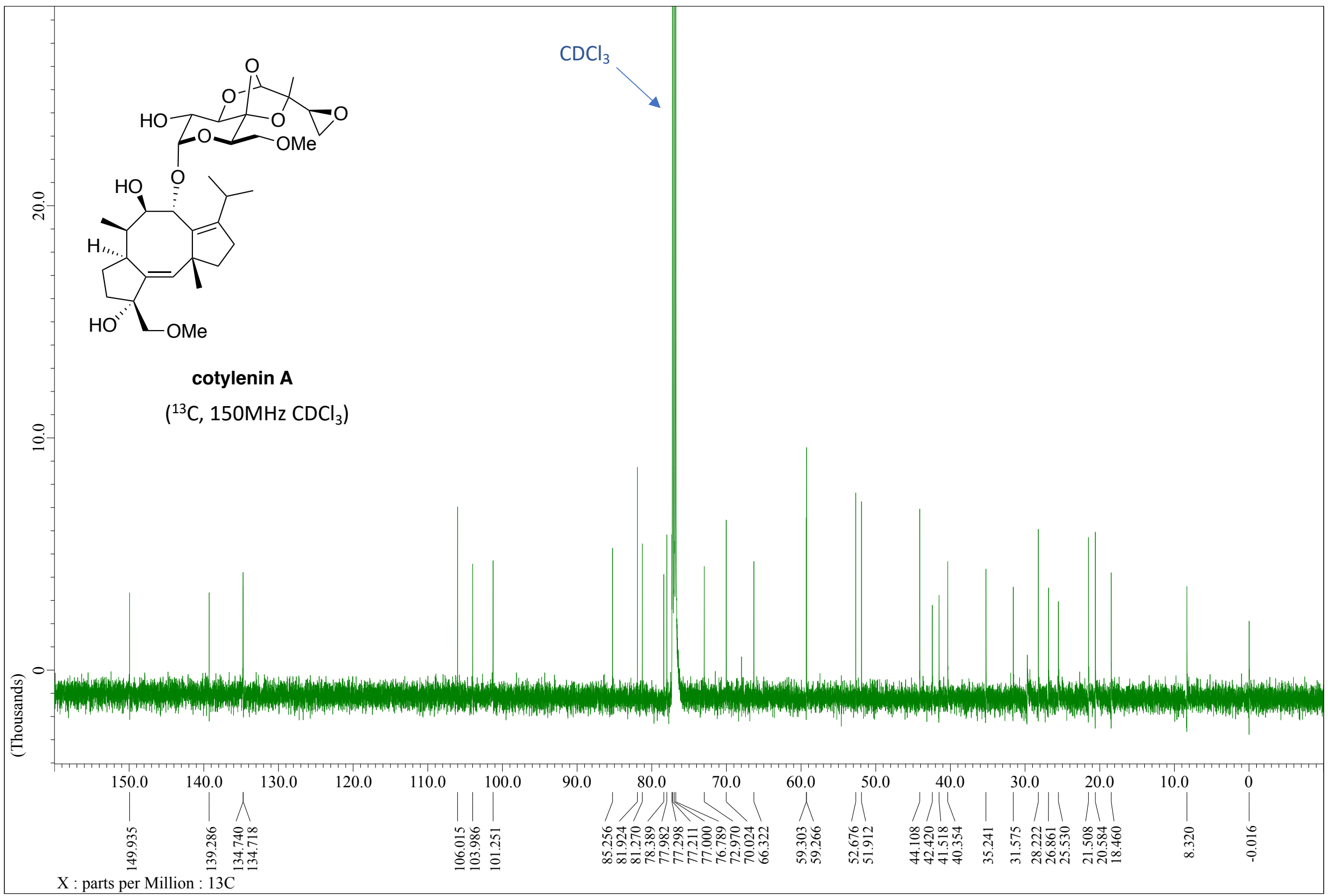

